\title{
Financial risk, vulnerability and equity of access to healthcare services in Kenya
}

Citation for published version (APA):

Njagi, P. M. (2021). Financial risk, vulnerability and equity of access to healthcare services in Kenya. [Doctoral Thesis, Maastricht University]. Boekenplan. https://doi.org/10.26481/dis.20210624nm

Document status and date:

Published: 01/01/2021

DOI:

10.26481/dis.20210624nm

Document Version:

Publisher's PDF, also known as Version of record

\section{Please check the document version of this publication:}

- A submitted manuscript is the version of the article upon submission and before peer-review. There can be important differences between the submitted version and the official published version of record. People interested in the research are advised to contact the author for the final version of the publication, or visit the DOI to the publisher's website.

- The final author version and the galley proof are versions of the publication after peer review.

- The final published version features the final layout of the paper including the volume, issue and page numbers.

Link to publication

\footnotetext{
General rights rights.

- You may freely distribute the URL identifying the publication in the public portal. please follow below link for the End User Agreement:

www.umlib.nl/taverne-license

Take down policy

If you believe that this document breaches copyright please contact us at:

repository@maastrichtuniversity.nl

providing details and we will investigate your claim.
}

Copyright and moral rights for the publications made accessible in the public portal are retained by the authors and/or other copyright owners and it is a condition of accessing publications that users recognise and abide by the legal requirements associated with these

- Users may download and print one copy of any publication from the public portal for the purpose of private study or research.

- You may not further distribute the material or use it for any profit-making activity or commercial gain

If the publication is distributed under the terms of Article $25 \mathrm{fa}$ of the Dutch Copyright Act, indicated by the "Taverne" license above, 


\section{Financial Risk, Vulnerability and Equity of Access to Healthcare Services in Kenya}

Njagi Purity Muthoni 
(c) 2021, Njagi Purity Muthoni

ISBN: 9789086665297

Publisher: Boekenplan, Maastricht

All rights reserved. No part of this publication may be reproduced, stored in a retrieval system, or transmitted in any form, or by any means, electronic, mechanical, photocopying, recording, or otherwise, without the prior permission in writing, from the author. 


\section{Financial Risk, Vulnerability and Equity of Access to Healthcare Services in Kenya}

\section{Dissertation}

to obtain the degree of Doctor at the Maastricht University, on the authority of the Rector Magnificus, Prof. Dr. Rianne M. Letschert

in accordance with the decision of the Board of Deans,

to be defended in public

on Thursday 24 June 2021, at 09:30 hours

by

Njagi Purity Muthoni 


\section{Supervisor:}

Prof. Dr. Wim Groot

\section{Co-supervisor:}

Dr. Jelena Arsenijevic

\section{Assessment Committee:}

Prof. Dr. Franziska Gassman (chair)

Prof. Dr. Jo Ritzen

Dr. Zina Nimeh

Prof. Dr. Khama Rogo, World Bank

Prof. Dr. Steven Koch, University of Pretoria 


\section{Table of Contents}

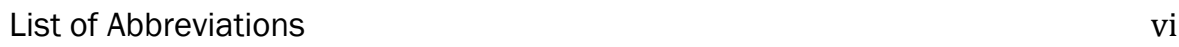

Chapter 1: General Introduction 1

Chapter 2: Variations in Catastrophic Health Expenditure, the Underlying Determinants, and Impoverishment in SSA:

A Systematic Scoping Review

Chapter 3 : Decomposition of Changes in Socioeconomic Inequalities in Catastrophic Health Expenditure in Kenya

Chapter 4: A Multilevel Analysis of Cost-Related Unmet Need for Healthcare Services in Kenya

Chapter 5: Impact of Household Shocks on the Utilisation of Healthcare Services in Kenya

Chapter 6: Conclusion and Policy Implications

References

Appendices

Summary

Impact Statement

Acknowledgements

Biography

Publications 


\section{List of Abbreviations}

\begin{tabular}{ll} 
AIC & Alkaike's information Criterion \\
ART & Antiretroviral therapy \\
ART & Assisted Reproductive Techniques \\
ATT & Average Treatment Effect on the Treated \\
BIC & Bayesian Information Criterion \\
CHE & Catastrophic Health Expenditure \\
CI & Concentration Index \\
CTP & Capacity To Pay \\
EAC & East Africa Countries \\
GDP & Gross Domestic Product \\
HISP & Health Insurance Subsidy Program \\
HIV & Human Immunodeficiency Virus \\
ICC & Intraclass Correlation Coefficient \\
KHHEUS & Kenya Household Health Expenditure and Utilization \\
KIHBS & Kenya Household Budget Survey \\
KNBS & Kenya National Bureau of Statistics \\
LMIC & Low and Middle-Income countries \\
MH & Mantel-Haenszel \\
MHI & Micro Health Insurance \\
MOH & Ministry of Health \\
NHIF & National Hospital Insurance Fund \\
NHIS & National Health Insurance Scheme \\
OOP & Out of pocket \\
PRISMA & Preferred Reporting Items for Systematic Reviews and Meta-Analyses \\
PS & Propensity Scores \\
PSM & Propensity Score Matching \\
SDG & Sustainable Development Goal \\
SDH & Social Determinants of Health \\
SSA & Sub Saharan Africa \\
TB & Tuberculosis \\
THE & Total Health Expenditure \\
UHC & Universal Health Coverage \\
WHO & World Health Organisation \\
\hline
\end{tabular}


Chapter 1 :

General Introduction 


\subsection{Scope of the dissertation}

The global call for universal health coverage (UHC) has spurred renewed attention among governments to prioritise the good health and well-being of the population. UHC exists when all people receive healthcare services without suffering financial hardship (WHO, 2006). In this way, it embodies equity in access to healthcare services and protection against financial risk. The WHO urges all countries to make UHC a political priority for the sake of peoples' health and sustainable development (WHO, 2017).

In Africa, several countries have identified UHC as a public health priority and have integrated it into their national health strategies. This is aimed at moving them closer to UHC through increasing access to healthcare services and financial risk protection (Musango et al., 2013). Despite the prioritisation of UHC by most low- and middle-income countries (LMICs), there is still a need to work on effective approaches for achieving UHC, especially with regard to quality and equity (Otieno \& Asiki, 2020). Kenya is not an exception and has made UHC a priority, through a commitment enshrined in the constitution of 2010 (Article 43) guaranteeing all citizens the "right to the highest attainable standard of health which includes the right to healthcare services" (GOK, 2010). This commitment is operationalised through the 2014-2030 health policy, which is underpinned by a rights-based approach to healthcare services delivery and focuses on ensuring equity $(\mathrm{MOH}, 2014 \mathrm{~d})$. One of the key obligations of the policy is to contribute to economic development as envisioned in the Kenya long-term development blueprint 'Vision 2030'. Good health is expected to reduce the economic and social vulnerability of poor households, thereby creating broad-based economic growth by providing a healthy and productive labour force for the nation (Friedrich-EbertStiftung, 2012).

Consequently, the reforms to ensure the right to health commitment by the government have taken place; key among them are the health-financing reforms to ensure equitable access to healthcare services for all. Kenya has been gradually introducing financing reforms since 1966, when the National Hospital Insurance Fund (NHIF) was launched. The NHIF scheme has since gone through numerous changes to include more benefits, extend to informal sector households and cover outpatient services (Muiya \& Kamau, 2013). Moreover, over the decades there have been on and off changes in user fees (with the introduction, then removal, then re-introduction, and reduction of user fees); however, user fees still exist (Munge \& Briggs, 2014). Evidence shows that these reforms have not led to the achievement of an equitable and accessible healthcare system for all Kenyans (Ilinca et al., 2019).

As per the 2010 constitution of Kenya, the healthcare system was devolved to the newly created sub-national government units as part of promoting equitable access to healthcare services (Williamson \& Mulaki, 2015). Currently, the national government takes leadership in policy formulation, and the county (regional) governments are responsible for the implementation of the policy activities (service delivery). This 
decentralisation is intended to reduce inequities of access in the healthcare system across populations and regions. Early results of the healthcare devolution highlight the commitment by the government to UHC through the pooling of resources, and investment in health infrastructure and personnel, although uncoordinated (Oketch, 2017).

While recognising the progress Kenya has made so far, challenges still lie ahead, particularly regarding equitable access for all regions and vulnerable population groups. Provision of healthcare in Kenya by the state has had mixed results, that have by and large negatively affected the poor and vulnerable societal members (Chuma et al., 2007). Although the poverty incidence in Kenya is below the average in Sub-Saharan Africa (SSA) (C. A. Awiti et al., 2018), around 36 per cent of the population live on less than KES 134 (US\$1.34) per day (Chhabra et al., 2018). Poverty and vulnerability remain major challenges, with almost one in every two Kenyans being trapped in a long-term, chronic and intergenerational cycle of poverty (GOK, 2011). Populations living in poverty experience multiple deprivations of income, housing and even basic services such as healthcare, and education (Mohanty, 2012). In Kenya, poverty has been reported to have a negative effect on the demand for modern healthcare services, in that the poor are less likely to consult a healthcare provider when sick relative to their non-poor counterparts (J. O. Awiti, 2014).

Due to the differences in the social status of vulnerable groups, potential gaps in healthcare often arise as they experience discrimination (Kilbourne et al., 2006). The vulnerability of the population to shocks is among the factors that have constrained poverty reduction in Kenya (Diwakar \& Shepherd, 2018). More than 61.9 per cent of Kenyan households have experienced some form of negative shocks with wide variations in the incidence of shocks across counties (KNBS, 2018a). Shocks constrain households' ability to invest in social welfare services such as health, and when they persist, coping becomes more difficult (Dercon, 2002). More importantly, shocks act as barriers to overcome poverty and may negatively affect health and lead to catastrophic payments for healthcare.

The social protection floors recommendation, 2012 (No. 202) emphasizes access to essential health services without financial hardship as a necessary social security guarantee under (ILO, 2012). Evidence suggests that social protection helps to protect households from the adverse effects of economic crises (WHO, 2015). In addition, effective social protection modalities have a high positive impact on the health outcomes of a population (Glennerster et al., 2009). While gaps in social health protection coverage and effective access are causes of health inequities, socioeconomic inequalities existing beyond the health sector contribute significantly to barriers in access to healthcare (Scheil-Adlung, 2014). Social protection and social investment could provide access to needed healthcare, ultimately reducing health inequalities.

Kenya has made progress in expanding the social protection mechanisms in the past few decades. Although the overall investment in social protection as a proportion of 
Gross Domestic Product (GDP) is only 1.3 per cent, it is higher than most countries in the SSA region. However, only 0.35 per cent proportion of GDP is invested in social assistance programmes including the Cash Transfer for Orphans and Vulnerable Children (CT-OVC), Persons with Severe Disabilities Cash Transfer (PwSD-CT), the Hunger Safety Net Programme (HSNP), Older Persons Cash Transfer (OPCT) and the senior citizens' programme for all older persons aged 70 years and above (GOK, 2017a). Other programs such as the social pension fund and health insurance are voluntary, based on an individual's ability to pay (E. Barasa et al., 2018). This provides limited coverage for the majority of the population that does not fall into the targeted groups.

A key aspect for achieving equity of access is a financial health protection mechanism, which ensures that all people in need of healthcare have effective access to adequate care (ILO, 2008). Financial risk protection is thus a component of broader social protection instruments needed to cushion the population from the adverse consequences associated with using healthcare services (Saksena et al., 2014). In general, financial reforms are considered as critical in fast-tracking improvement in overall healthcare of populations and progress towards UHC (Chu et al., 2019).

The majority of households in Kenya lack financial risk protection. Despite insurance coverage having increased over time, only about 21.3 per cent of the population have some form of health insurance. Rural households are at a higher risk of being uninsured, with only 13.3 per cent of the population with health insurance relative to 29.2 per cent in urban areas (KNBS, 2018a). Moreover, there are psychosocial and economic factors such as trust, risk-preferences, inadequate finances, cost of healthcare, and even perceived health status that are reported to influence insurance uptake (Adebayo et al., 2015; Otieno et al., 2019). The lack of financial protection has increased the risk of households to catastrophic health payments with the poorest households being disproportionately affected. Furthermore, between 1 and 1.1 million individuals are pushed into poverty due to out of pocket (OOP) payments (Salari et al., 2019). Equally, the unmet need for healthcare is reported to be 12.7 per cent, while 21.4 per cent of the population report cost as the main barrier preventing them from seeking care when needed $(\mathrm{MOH}, 2014 \mathrm{a})$.

Evidence suggests that reforms such as those in healthcare might fail due to poor formulation and implementation, and for those which are successful, there is no certainty that the success will last (Fullan, 2000). For instance, some studies have shown a negative impact of insurance on equity in the distribution of healthcare services (Blanchet et al., 2012; Waters, 2000). In contrast, other experiences from countries such as Ghana and Burkina Faso have shown that devolution in itself does not essentially lead to improvements in efficiency or equity (Couttolenc, 2012; Zon et al., 2019). Conversely, in terms of equity, even the best-intentioned health schemes could be inequitable (Kent Ranson et al., 2006). As many LMICs strive for equitable, accessible and inclusive healthcare, people's right to health may often go unnoticed if health policies are not reoriented to address the needs of the most vulnerable (MacLachlan et al., 2012). 
This dissertation is inspired by the public health discourse on how best to ensure equity in access to healthcare in Kenya while guaranteeing effective protection for the poorest and most vulnerable against the financial risk of ill health. This will contribute further to the limited empirical evidence in SSA to comprehensively inform health and redistributive policies. By gaining a better understanding of the extent of financial risk protection and the associated socio-economic disadvantages that exacerbate risks, policymakers could better formulate approaches that optimise the existing financial risk protection and equity of access mechanisms, and also policies that are aimed at strengthening the financial well-being of the households.

\subsection{Equity in healthcare}

The health policy in Kenya is driven by the recognition of the fundamental human rights, including the right to health as enshrined in the 2010 constitution. In this case, it embraces the principles of protection of the rights and fundamental freedoms of specific groups of persons by focusing on equity and people centeredness $(\mathrm{MOH}$, 2014d). Therefore, to effectively study the healthcare system, it is paramount to understand the principle of equity that underpins the health policy and system in Kenya.

Equity is an ethical concept grounded on values of distributive justice (Braveman \& Gruskin, 2003; Waters, 2000). Equity has for decades been a stated or implied goal of health policy in many countries and international health organisations (Bonnefoy, 2010; Vedom \& Cao, 2011). Equity has two dimensions: horizontal equity, that refers to equal access for equal need (Hodgson, 2010) and vertical equity, which refers to appropriate unequal treatment of unequals (Culyer, 2001). The Millennium Development Goals (MDGs) were faulted for their failure to address equity by neglecting the most vulnerable populations (Rodney \& Hill, 2014). Consequently, health equity is a priority in the post-2015 sustainable development agenda, amongst other major health initiatives (Hosseinpoor et al., 2015). The principle to achieving health equity is said to be the attainment of 'highest standard of health for all' through the elimination of healthcare disparities and its cause, with a special focus on those at greatest risk of poor health based on their socio-economic circumstances (Braveman, 2006, 2014). Thus, policymakers view health equity as an integral part of UHC (Bonnefoy, 2010; Cissé et al., 2007). The aim of UHC is to ensure that healthcare benefits are distributed on the basis of the need for care and not the ability to pay (WHO, 2006; Chuma et al., 2012). In this way, UHC represents a mechanism to achieve horizontal equity in healthcare.

Health finance reforms are viewed as an avenue from which UHC can be fasttracked given the capacity to reduce financial barriers amongst the underprivileged, and create equitable access to healthcare services (Gelormino et al., 2011). In most cases, in low-income countries even when UHC is proclaimed many services are still paid through OOP payments, or the quality of care for services that are free of charge is inadequate (Yu et al., 2008). There is a growing global need to reduce financial barriers to healthcare with particular emphasis on high priority services and vulnerable groups 
(Witter et al., 2009). Financial barriers are reported to limit access to healthcare services in both high- and low-income countries (Aaltonen et al., 2015; Gulliford et al., 2002).

Financial risk protection is concerned with safeguarding people against the financial hardship associated with paying for healthcare services (Saksena et al., 2014). To monitor financial risk protection, two indicators are commonly used; one is the incidence of catastrophic payments, and the other is impoverishment (Quintal, 2019; WHO \& World Bank, 2017). Catastrophic expenditure is experienced when OOP expenses exceed a percentage of household consumption while impoverishment is when households are pushed into poverty by healthcare payments (Adam Wagstaff \& Eozenou, 2014). Lowering financial barriers is one way to more equitable access to healthcare (Allin, Hernández-Quevedo, et al., 2010). Therefore, the unmet need is a way to gauge equity of access to healthcare services (OECD, 2011).

Striking evidence of inequities in the access to and utilisation of healthcare resulting from income differences and rural-urban locations have been identified in selected African countries (ECA, 2009). Furthermore, the reasons for why care is not sought when illness occurs have been shown to vary according to income groups as well as the health payment approach (Harris et al., 2011). Many African governments are grappling with the challenge of how to devise health policies and healthcare systems that can ensure equity of access to adequate healthcare (Mutangadura et al., 2007). In this regard, many countries are considering to reform the health financing system which is expected to have a substantial impact on equity of health financing; however, the problems are daunting in LMICs (Ensor \& Ronoh, 2005) surmising due to the competing development priorities.

\subsection{The healthcare system in Kenya}

\subsubsection{Overview of the healthcare system}

The Kenyan healthcare system comprises of the public system, with major players including the Ministry of Health $(\mathrm{MOH})$ and parastatal organisations, and the private sector, which includes private for-profit, Non-Governmental Organisations(NGO), and Faith Based Organisations(FBO) facilities (NCAPD et al., 2005). When Kenya attained independence in 1963, the public healthcare system was centralised consisting of various levels of health facilities which included national referral hospitals, provincial general hospitals, district hospitals, health centres and dispensaries. However, as a result of the implementation of the 2010 constitution of Kenya, the responsibility for healthcare services has been transferred to the newly formed county governments effective from 2013. The world bank argues that the Kenya devolution is complex and logistically ambitious because it transfers power in terms of decision making to new units of governance that do not have existing revenue sources (World Bank, 2012).

In the new healthcare structure, besides policy formulation, the national level is responsible for national referral hospitals which are the apex of the healthcare system, 
providing sophisticated, diagnostic, therapeutic and rehabilitative services (NCAPD et al., 2011). The county government healthcare services are organised around three levels of care, namely the community level, primary care level and country referral system. The community-level focus is to organise appropriate demand for services at the community, while the primary care level focus is to provide appropriate demanded services and delivery, especially preventive healthcare and curative services. Therefore, the primary care level comprising of all dispensaries, health centres and nursing homes is the interface between the community and the rest of the healthcare system. The county referral system comprises of all the county referral hospitals providing specialised medical services within the region (county) $(\mathrm{MOH}, 2014 \mathrm{~b})$.

Currently, Kenya's health aspirations are articulated in various national documents from which the constitution provides the overarching legal framework to a rights-based approach to healthcare services delivery. Kenya Vision 2030, which is the long term development blueprint, seeks to achieve an efficient and high-quality healthcare system, with one of the flagship projects being to promote equity in Kenya's healthcare financing (GOK, 2007). The 2014-2030 healthcare policy aligns with the constitution and Vision 2030 by providing policy principles and orientations that facilitate the development of a comprehensive healthcare system ( $\mathrm{MOH}, 2014 \mathrm{~d})$. Further, the national health sector strategic plan (NHSSP) seeks in the medium term to accelerate attainment of UHC through improving services, scaling up services and reducing financial implications of accessing healthcare $(\mathrm{MOH}, 2013 \mathrm{~b})$. Finally, the presidency has reassured the commitment to improving healthcare through the "The Big Four" priorities which intend to achieve 100 per cent UHC by increasing the uptake of the health insurance fund especially among the vulnerable groups (GOK, 2017b)

\subsubsection{Healthcare financing}

Health financing systems are intended to improve health outcomes, provide financial risk protection against catastrophic health costs and improve access to healthcare services (Kutzin, 2013). However, it's noted that there is no 'best model' for health financing as the starting point and the context is unique for each country, with the emphasises on homegrown strategies (Kutzin et al., 2016; WHO, 2010). Financing sources for health are categorised into taxation, social health insurance, private health insurance and OOP payments.

Kenya has had a predominately tax-funded healthcare system since independence in 1963. In the first two decades after independence (70s and 80s), Kenyans had free access to healthcare services at all public health facilities funded through tax revenues (Chuma \& Okungu, 2011). The NHIF was established under the Ministry of health in 1966, to provide health insurance to workers in the formal sector, and later in 1972 it was amended to include the informal sector on a voluntary basis $(E$. Barasa et al., 2018). In 1989, during the structural adjustments program, the government introduced outpatient and inpatient user charges at all public sector 
facilities except dispensaries to mobilise additional resources. In 1990, reforms to the NHIF were introduced that include graduated premium(premium based on income scale) while user charges were accompanied by waivers based on ability to pay 'suspension'. Three months later, due to the marked fall in facility attendance, the user fees were suspended.

In 1992, user fees were reintroduced progressively from the national hospital to district and health centres, while the waiver system was expanded (Swamy, 1994). In 1998 the NHIF was transformed into an autonomous entity away from the MOH through the NHIF Act (Munge et al., 2015). Later in 2004, user fees were suspended and replaced with the '10/20 policy' which was a standard registration fee of Kenyan shillings $10-20(0.10 \$-0.20 \$$ at current exchange rate) for children under five and for other special conditions (Chuma \& Okungu, 2011). In the same period a proposal for a national health insurance scheme (NHIS) was developed which was highly controversial, but nevertheless passed in parliament; however, the president declined to assent to the bill citing affordability and sustainability challenges. NHIS discussions were reinitiated in 2007 , leading to a proposal to develop a draft health financing strategy in 2009 to guide the country towards UHC (Abuya et al., 2015).

In 2013, all user fees in public health centres and dispensaries, and maternity services in all public health facilities were abolished to promote equity in access to essential healthcare services (Maina \& Ongut, 2014). In 2015, the NHIF was extended to cover outpatient services in various public health hospitals and the contribution was increased to cater for rising costs of healthcare (Mbau et al., 2020). The NHIF covers services that are provided by both public and private (for profit and not for profit) healthcare services through an accreditation model based on the provider type. However, the private healthcare package is limited, covering mainly bed and drug expenses (Suchman, 2018). Conversely, user fees still remain the higher levels of care, particularly in hospitals that offer specialised treatment, and for services that may not be covered by the health insurance (Kabia et al., 2019). These user fees are not standard and vary by the level of health facility and the type of treatment sought. Recent evidence suggests that Kenyans pay an average OOP of 7,990 shillings ( $\$ 79.9$ at current exchange rate) per year (Salari et al., 2019). Table 1.1 summarises the NHIF and user fees reforms in Kenya. 
Table 1.1: Summary of NHIF and User Fee Reforms in Kenya

\begin{tabular}{|c|c|}
\hline $\begin{array}{l}\text { National Hospital Insurance Fund } \\
\text { (NHIF) }\end{array}$ & User Fees Charges \\
\hline $\begin{array}{l}\text { 1966-NHIF was established to } \\
\text { cover the formal sector }\end{array}$ & 1963-Free primary healthcare services \\
\hline $\begin{array}{l}\text { 1972-NHIF reformed to include the } \\
\text { informal sector }\end{array}$ & $\begin{array}{l}\text { 1989-Introduction of inpatient and } \\
\text { outpatient user charges except for } \\
\text { dispensaries }\end{array}$ \\
\hline $\begin{array}{l}1990-\text { NHIF reforms to include } \\
\text { graduated premium }\end{array}$ & $\begin{array}{l}\text { 1990-User fee accompanied by waivers } \\
\text { based on ability to pay. Three months later } \\
\text { user fees were suspended }\end{array}$ \\
\hline $\begin{array}{l}\text { 1998-NHIF transformed into an } \\
\text { autonomous entity away from the } \\
\text { Ministry of Health(MOH) }\end{array}$ & 1992-User fees reintroduced progressively \\
\hline $\begin{array}{l}\text { 2004-Proposal for national health } \\
\text { insurance scheme (NHIS) } \\
\text { developed, president, declined to } \\
\text { assent to the bill }\end{array}$ & $\begin{array}{l}2004-\text { User fees suspended and replaced } \\
\text { with the Kenya shillings "10/20" } \\
\text { registration fee }\end{array}$ \\
\hline $\begin{array}{l}2007-\text { NHIS discussion reinitiated } \\
\text { leading to a health financing } \\
\text { strategy in } 2009\end{array}$ & $\begin{array}{l}\text { 2013-User fees in public health centres } \\
\text { and dispensaries abolished. } \\
\text { Free maternity services in all health } \\
\text { facilities (including hospitals) }\end{array}$ \\
\hline $\begin{array}{l}\text { 2015-NHIF extended to cover } \\
\text { outpatient and premiums reviewed } \\
\text { upwards }\end{array}$ & $\begin{array}{l}\text { To Date-User fees still exist in some levels } \\
\text { of the healthcare system especially } \\
\text { hospitals }\end{array}$ \\
\hline
\end{tabular}

In 2014, the government rolled out the health insurance subsidy program for the poor (HISP), a pilot program which extended financial risk protection to the poorest citizens. The objective of HISP was to increase prepaid health insurance coverage by ensuring that the state covered the full insurance premiums for the beneficiaries to access the full benefits of the health insurance cover (MOH, 2013a). Later in 2016, HISP was scaled up to include more vulnerable households by providing comprehensive inpatient and outpatient services (E. W. Barasa et al., 2018).

Health financing reforms continue to date with some being as recent as 2018 when the government launched an ambitious plan to accelerate achievement towards UHC. This started with a pilot in four counties and subsequently was scaled up to other counties in 2019/20. 
Healthcare in Kenya is financed from government expenditure, private sector (including household expenditure, both out-of-pocket and formal and non-formal insurance spending) and donor funding (Mugo et al., 2018). Over time private sector financing has been declining while public sector financing has remained constant. For instance, between 2001/02 and 2009/10, private sector expenditure decreased from 54 per cent to 37 per cent, while public funding remained at 29 per cent of total health expenditure(THE) (MOH, 2010). By 2013, Kenya's THE had increased to about US $\$ 50$ total per capita expenditure surpassing the WHO benchmark of US $\$ 42$ and exceeding that of other East Africa Countries(EAC). However, the health sector spending accounted for only six per cent of total government expenditure which was the lowest share in the EAC (World Bank, 2014). This is despite Kenya being the fourth largest economy in SSA and playing economic dominance in the EAC region (Kimenyi et al., 2015). By 2019, the total government health expenditure as a proportion of both national and county government budget was 6.7 per cent. The county governments are highly dependent on national resource transfers given that they have limited resource streams and taxation capacity; however, they have autonomy over planning and managing the resources (Dutta et al., 2018). OOP expenditure continues to be high, constituting 32 per cent of the total health budget (N. David \& Wanjala, 2020). Furthermore, OOP payments in Kenya have been shown to be regressive with all other payments being proportional (Munge \& Briggs, 2014).

Despite all these reforms, Kenya's financing system is faced with numerous challenges including the access to individuals and household being fragmented by coverage scheme, while the poor and vulnerable are excluded. For instance, the NHIF is criticised for not being able to effectively reach out to the majority of Kenyans households and individuals especially, the poor and those in the informal sector (Luoma et al., 2010). Furthermore, the NHIF is a membership-based scheme and not a state funded social scheme. Even with the HISP being a programme targeted at the poor and most vulnerable, recent evidence shows that the beneficiaries have not reaped the full benefits due to other access barriers including delayed insurance disbursements to facilities where HISP beneficiaries seek care, informal fees and continued charging of user fees despite abolition, medical supplies stock-outs and shortage exposing the poor to OOP payments_(Kabia et al., 2019).

\subsection{Relevance and research gap}

Many governments have made commitments to tackle inequities in access, but making this policy operational has been difficult without a clear picture of what is currently known about equity in access to healthcare services (Goddard \& Smith, 2001). Further, measuring health equity is argued to be a critical step to promote opportunities for all people regardless of their socio-economic background (Dover \& Belon, 2019). Identifying health inequities for each population and analysing their extent and causes is proposed as the first key step in the development of national health equity strategies 
(Friedman, 2015). Although there has been increased knowledge on inequities in healthcare and their pathways, there is limited empirical evidence especially in LMICs of which policies can reduce the inequities in specific contexts (Rasanathan \& Diaz, 2016).

Despite the several existing mechanisms to address inequity in Kenya, the healthcare system has been criticised for regional discrepancies in the health service distribution, disparities in resource allocations and inequitable access to quality healthcare services (Nyanjom, 2006). Disparities exist due to the diverse geography, socio-economic status and other social determinants of health across the regions (counties) (Achoki et al., 2019). Moreover, the Kenyan healthcare system is reported to be inequitable, and benefits are not distributed according to need (Chuma et al., 2012). Further assessment of the healthcare system has shown regional variations in health infrastructure and even health workforce. For instance, while the national average of health facilities to population size was 2.4 (per 10,000 persons), the regional variations ranged between $<1$ to 3.5 per 10,000 persons $(\mathrm{MOH}, 2014 \mathrm{c})$. There is an unequal distribution of health workers by urban/rural, and by regions and level of care; and an absolute shortage of healthcare workers with a provider-population ratio of 1.69/1000 (for all cadres of providers) although relatively high for countries in the SSA region (Luoma et al., 2010).

Evidence shows that there exist wide variations in unmet needs across counties in Kenya ranging between 4.1 per cent and 40.4 per cent while the per capita utilisation rate of healthcare is reported higher among the well-off relative to the poor households $(\mathrm{MOH}, 2014 \mathrm{a})$. User fees and OOPs have had negative effects on the utilisation of healthcare in Kenya. The majority of the population cannot afford to pay for healthcare, and the poor are unlikely to use healthcare services while disparities exist according to geographical regions (Chuma \& Okungu, 2011). Additionally, the percentage of households experiencing catastrophic health expenditure ( $\mathrm{CHE}$ ) (considering a 40 per cent threshold) increased from 6.2 per cent in 2013 to 7.1 per cent in 2018 . This is an indication that the healthcare reforms have not been successful in cushioning the population from financial risks of ill health (Salari et al., 2019). These and many other examples depict the complexity of inequities in the Kenyan healthcare system that continues to deny access to a large population, especially the most vulnerable.

There are several proposed measures used to quantify different perspectives of health inequity and financial risk protection (O'Donnell, 2007); however, many studies focus on the analysis of not more than one measure. This way, other perspectives of the healthcare system are not captured. It is thus imperative to use more than one measure for a holistic and robust assessment of healthcare inequities (Alonge \& Peters, 2015). For instance, the incidence of $\mathrm{CHE}$ commonly used to monitor the financial risk protection is based on OOP payments; hence, a low incidence could be related to people refraining from access due to catastrophic costs (Adam Wagstaff et al., 2018). Moreover, $\mathrm{CHE}$ only observes people who actually have used healthcare, and not those who have refrained from doing so. The incidence does not account for disparities between the poor 
and rich; hence, the measurement of the socio-economic inequalities would thus provide insights on whether the gap is growing or reducing. Conversely, the unmet need monitor equity of access amongst populations which are not able to seek care when they need it for various reasons (Allin \& Masseria, 2009b). Nevertheless, an average measure of unmet needs may mask variations across or within diverse regions. Nonetheless, even with the ability to pay for healthcare when needed, shocks reduce the financial well-being of the households (WHO, 2015) and may constrain their decisions to seek healthcare when necessary.

It is against this backdrop that this dissertation looks at assessing various perspectives of equity in access to healthcare services. It not only focuses on the impact of lack of financial risk protection for individuals who use healthcare services but also for those who forgo healthcare services due to financial barriers. The analysis extends beyond the general measurement of financial risk protection to the extent of socioeconomic disparities in financial burden and explores cost barriers to access across and within regions and to what extent risks confound the ability of households to invest in healthcare. Getting to the depth of financial risk protection and its relation to equity of access to healthcare in Kenya is therefore timely and critical in shaping the redistributive policies going forward. This would help to inform the current health financing reforms in Kenya to better cater for the needs of the most vulnerable population groups and to develop actions towards decreasing the existing inequities. This study adds to the inequity literature by exploring the nuances in the measurement and factors that drive inequities across various population groups in Kenya.

\subsection{Research questions and data sources}

Despite Kenya being among the first SSA countries to implement a national insurance scheme in the 60s, followed by many other financial reforms, inequities continue to manifest themselves in the healthcare system. Particularly, access across different groups of the population within the country varies. To date, Kenyan households continue to experience catastrophic payments, with more than one million people annually pushed into poverty due to healthcare payments (Salari et al., 2019). This study explores inequity in access to healthcare services by focusing on affordability as an entry to the healthcare system. In doing so, the study responds to the following questions:

\section{Question 1: What is the extent, variations and the underlying determinants of CHE across Sub-Saharan African countries?}

People in poor countries tend to have less access to healthcare services than those in better-off countries, and within countries, the poor have less access to healthcare services (O'Donnell, 2007; D. H. Peters et al., 2008). This is largely due to the numerous barriers that hinder access (Jacobs et al., 2012) including financial costs, opportunity costs in travel, waiting time, availability, affordability and the perceived quality of services (Odaga, 2004). CHE is widespread and varies in different countries, depending on their healthcare system, and social structure (Xu et al., 2003a). Poorest 
households are said to be the most at risk (Haghparast-Bidgoli et al., 2015). Variations in $\mathrm{CHE}$ and their determinants provide evidence on the existing disparities in financial risk protection based on the diverse context of healthcare systems in various countries.

Understanding the extent of variations in CHE will not only shed light on the gravity of inequities in Kenya relative to other countries in the region, but also draw lessons that could inform policy reforms. Therefore, this study applies a systematic review approach to assess the evidence on catastrophic payments in SSA from several databases including PubMed, EBSCO [EconLit, Psycholnfo, CINAHL], Web of Science, Jstor and virtual libraries of the World Health Organizations [WHO], and the World Bank. The primary outcome of interest is the incidence of $\mathrm{CHE}$, while the secondary outcome is the associated risk factors. Following the Preferred Reporting Items for Systematic Reviews and Meta-Analyses (PRISMA) tool in systematic review approach helps to synthesise the existing knowledge with a focus on how catastrophic health payment has been contextualised and examine the scale of the incidence in SSA and the associated determinants.

\section{Question 2: What is the extent of socio-economic inequalities in CHE, the associated determinants and the changes over time in Kenya?}

Studies have shown that inequalities in CHE exist, and could be disproportionately concentrated amongst particular groups (Kien et al., 2016). In Kenya, households spend about a tenth of their budgets on healthcare through OOP payments, and in absolute terms, a rich household spends much more of their household budget on healthcare than poorer households (Chuma \& Maina, 2012). The poorest households are seen to incur high costs on healthcare relative to their total expenditure (Chuma et al., 2007). Conversely, inequalities in $\mathrm{CHE}$ exist and are disproportionately concentrated amongst the less well-off (E. Barasa et al., 2017). This study provides context on the factors that sustain inequalities in $\mathrm{CHE}$ hence nudge policymakers to formulate appropriate measures that protect the most vulnerable.

Although inequalities in CHE are reported to exist in Kenya, no study has assessed changes in CHE inequalities over time, and it is not clear what factors sustain the inequality. The concertation index is applied to measure the socio-economic inequality in CHE using data from two rounds of the Kenya household health expenditure and utilisation (KHHEUS) survey (2007 and 2013). Households are classified into socioeconomic quintiles using per capita consumption expenditure, and following the Wagstaff (2003) approach, the concentration index of CHE is decomposed to assess the relative contribution of its determinants. Finally, the Oaxaca-type decomposition is applied to measure the change in inequality in CHE between 2007 and 2013.

\section{Question 3: Do sub-region variations influence the unmet need for healthcare services due to cost?}

Prompt access to primary healthcare before the onset of severe illness is vital to improve morbidity and mortality rates (Rees et al., 2016). When there are no financial barriers to access healthcare, then CHE can be prevented, and universal coverage can 
be achieved (Ezat Wan Puteh \& Almualm, 2017). Despite CHE being a measure of the extent of financial risk protection of a system, its analysis may create the impression of a greater degree of financial risk protection given that it does not include those who do not access healthcare services (Mchenga et al., 2017). Therefore, unmet needs for healthcare is one of the other methods of gauging equity of access to services (OECD, 2011). Assessing the regional variation in unmet needs is useful to inform the prioritisation of healthcare services in counties that are lagging behind, as well as to take lessons from the counties performing well.

In Kenya, wide variations in unmet healthcare needs are reported across counties ranging between 4.1 per cent to 40.4 per cent, with the cost being one of the top reasons why people forgo care ( $\mathrm{MOH}, 2014 \mathrm{a})$. This question is answered using the 2013 Kenya household health expenditure and utilisation survey (KHHEUS). Self-reported unmet need due to lack of money and high costs of care is used to compute the outcome of interest. A multilevel regression model is applied to assess the determinants of costrelated unmet need, confounding for the effect of variations at the regional level.

\section{Question 4: What is the effect of household shocks on the utilisation of healthcare services in Kenya?}

Shocks and stresses create context-specific impacts on individual health, wellbeing and systems of care that reveal weaknesses in resilience (L. Clarke \& Masson, 2017). Population in many developing countries are susceptible to poverty due to shocks (Atake, 2018). For many households with limited or no financial risk protection, negative income shocks are likely to have an effect on healthcare (Baulia, 2018). Shocks are likely to lead to poverty and in turn, worsen health status (OECD \& WHO, 2003). In Kenya, three out of every five households have experienced some form of shock, with a higher incidence in rural areas. Formal mechanisms that cushion for shocks are largely absent in Kenya, with only 21.3 per cent of the population having some form of health insurance (KNBS, 2018a). In Kenya, poor households may forego healthcare services due to costs, while high-income households may also experience significant financial burden (llinca et al., 2019). Shocks may limit the financial capabilities of households and subsequently influence the uptake of healthcare services. Understanding how economic shocks interplay with the utilisation of healthcare services would provide insights to policymakers for formulating interventions that can build the financial resilience of the households and offer cushion against financial risks.

Using the cross-sectional Kenya household budget survey(KIHBS) 2015/16, this dissertation examines the impact of household shocks on the utilisation of healthcare services. Chapter 5 employs the propensity score matching (PSM) approach to construct a quasi-experimental design by creating a counterfactual to compare the effects between households that had and had experienced a shock. 


\subsection{Outline of the dissertation}

This dissertation is organised into six main chapters structured as follows: Chapter 2 provides a synthesis on the extent of $\mathrm{CHE}$, the existing variations in its distribution, and the known risk factors across Sub-Saharan African countries. Chapter 3 addresses the second question by focusing on the socio-economic inequalities in CHE across two time periods, and the associated drivers of inequalities while also examining the change in CHE inequality over time. Chapter 4 discusses the third question by exploring the scale and determinants of unmet needs due to cost barriers across regions in Kenya, while Chapter 5 focuses on the impact of household asset and income shocks on the uptake of healthcare services. Finally, Chapter 6 summarises the key findings, conclusions and policy implications from all the chapters. 


\section{Chapter 2 :}

\section{Variations in Catastrophic Health Expenditure, the Underlying Determinants, and Impoverishment in SSA: A Systematic Scoping Review}

\section{Based upon:}

Njagi Purity, Arsenijevic Jelena, Groot Wim 2018; Understanding variations in catastrophic health expenditure, its underlying determinants and impoverishment in Sub-Saharan African countries: a scoping review. BMC Systematic Reviews Journal. https://doi.org/10.1186/s13643-018-0799-1 


\section{Abstract}

Background: To assess the financial burden due to OOPs, two mutually exclusive approaches have been used: CHE and impoverishment. SSA countries primarily rely on $\mathrm{OOP}$ and are thus challenged with providing financial risk protection to the populations. To understand the variations in CHE and impoverishment in SSA, and the underlying determinants of $\mathrm{CHE}$ a systematic review of the existing evidence was conducted.

Methods: This review followed the PRISMA approach. A search was conducted in several databases including PubMed, EBSCO [EconLit, Psycholnfo, CINAHL], Web of Science, Jstor and virtual libraries of the World Health Organizations [WHO], and the World Bank. The primary outcome of interest was catastrophic health expenditure/impoverishment, while the secondary outcome was the associated risk factors.

Results: Thirty-four (34) studies that met the inclusion criteria were fully assessed. CHE was higher among West African countries, and among patients receiving treatment for HIV/ART, TB, Malaria and Chronic illnesses. Risk factors associated with CHE included household economic status, type of health provider, socio demographic characteristics of household members, type of illness, social insurance schemes, geographical location and household size/composition.

Conclusion: This review demonstrated that CHE and impoverishment is pervasive in SSA, and the magnitude varies across and within countries and over time. Socioeconomic factors are seen to drive CHE with the poor being the most affected, and they vary across countries. This calls for intensifying health policies and financing structures in SSA, to provide equitable access to all populations especially the most poor and vulnerable. There is a need to innovate and draw lessons from the 'informal' social networks/schemes as they are reported to be more effective in cushioning the financial burden. 


\subsection{Introduction}

Financial barriers are a key limitation to access healthcare services in LMICs (Bright et al., 2017; Fortney et al., 2011). Financial barriers are usually related to OOP patient payments and their impact on household budget (Murray et al., 2003). Two main approaches are used to assess the financial barriers: catastrophic health expenditure that occurs when OOP payment equals or exceeds a pre-specified threshold of household expenditure or capacity to pay (Ezat Wan Puteh \& Almualm, 2017; Xu, 2005); and impoverishment that occurs when the average household consumption after healthcare payment is below the pre-specified international or national poverty line (Limwattananon et al., 2007).

The incidence of catastrophic payments is reported to be higher in low-income countries that rely on OOP, and lower in countries that have some prepayment mechanisms (Ezat Wan Puteh \& Almualm, 2017; Murray et al., 2003). While impoverishment is usually reported in LMICs, catastrophic payments also exist in higherincome countries, and are slightly concentrated among the less well-off (Ezat Wan Puteh \& Almualm, 2017; van Doorslaer et al., 2007). This trend is also observed in African countries. Studies in SSA countries have shown that inequities in access exist as a result of income differences and the level of OOP within the country. The proportion of households facing catastrophic healthcare payments has been shown to vary widely between countries (Leive, 2008; Mutangadura et al., 2007; Xu et al., 2003a). WHO argues that when people suffer financial hardship due to OOP, it's impossible to get closer to UHC due to the high risk of catastrophe and impoverishment (WHO et al., 2010). Moreover, UHC aims to ensure that healthcare benefits are distributed on the basis of need for care and not on ability to pay (Chuma et al., 2012). The burden of OOP payments has encouraged SSA countries to use different financial arrangements to prevent catastrophic payments (Mills et al., 2012). One of them is introduction of insurance systems with universal population coverage (Verguet et al., 2016) and another is removal of user fees. There is also a trend by governments to move out of the OOP payments that are considered to impoverish those who are already poor (Akinkugbe et al., 2012).

Given the over reliance on OOP payments in most of SSA countries, and with most countries having inadequate social insurance schemes, there is a strong need to evaluate systematically the existing evidence on financial inequity in access to healthcare. Furthermore, the effectiveness of the health financing system is seen through protecting people against the risk of becoming poor, while enabling them to make use of services (Kutzin \& others, 2008). In addition, WHO underscores healthcare financing as one of the crucial components of the broader efforts to ensure social protections (WHO, 2010). Furthermore, access to essential healthcare services is one of the key basic social security guarantees(ILO, 2012).

Systematic reviews synthesize evidence given their clearly formulated structure and the methodological rigor (Khan et al., 2003). Several studies have reviewed the variations in health indicators in SSA, however most of these have focused on health 
status, service coverage and utilization indicators like mortality rates, incidence/ prevalence of diseases (Burgert-Brucker et al., 2015; Gribble \& Haffey, 2008). There have been few reviews that focus on unequal access to healthcare due to financial barriers (V. A. Welch et al., 2013). A lack of systematic reviews in this area is perceived by policy makers as a limitation in decision making and developing new strategies (V. Welch et al., 2010). While there are several systematic reviews on catastrophic payments and impoverishment in LMICs (Alam \& Mahal, 2014; Tanimura et al., 2014) very few have incorporated literature from SSA, and those that have done so have included only one or two countries from the SSA region (Ezat Wan Puteh \& Almualm, 2017; Jaspers et al., 2015). A few other reviews that have been conducted are disease specific (Goryakin \& Suhrcke, 2014; Kankeu et al., 2013; Russell, 2004) and do not review the $\mathrm{CHE}$ risk factors. To our knowledge there is currently no systematic and/or scoping review that examines the scale and variations of $\mathrm{CHE}$ and impoverishment across SSA countries. To understand the scope and nature of the studies conducted in SSA we apply a scoping review approach. Scoping reviews are considered appropriate in that they not only bring together the available evidence but also provide broader synthesis of the evidence (M. D. J. Peters et al., 2015).

The aim of this review is to provide an overview of the magnitude and distribution of catastrophic health expenditure and impoverishment due to OOP for healthcare across SSA countries. Furthermore, it also looks into the determinants of CHE across SSA countries. This will not only highlight the scale of the problem but also identify any gaps that could potentially strengthen future research in CHE or impoverishment in SSA countries. The findings will help in developing effective health policies (Séne \& Cissé, 2015), that are more targeted, prioritise the vulnerable populations and address key risk factors. In addition, the findings could help to inform strategic health financing priorities of SSA member states by development partners/regional blocs like the African union (AU), WHO, and World bank amongst others that invest in health initiatives in the region.

This review therefore responds to the research question: what variations exist in the distribution of $\mathrm{CHE}$ and/or impoverishment, and the associated risk factors across SSA countries? The chapter continues with the method section (searching strategy and study selection) followed by the result section and discussion with conclusions.

\subsection{Methods}

This scoping review is based on the PRISMA statement that provides key items considered to be essential and minimum components of a systematic review or metaanalysis protocol (Moher et al., 2015) was applied to guide the screening and eligibility of the studies. 


\subsubsection{Search strategy and inclusion criteria}

\section{Inclusion and exclusion criteria}

This review included studies that focused on all population groups including vulnerable groups like people living with disability, the elderly or children in both rural and urban settings. Studies with the primary aim of assessing catastrophic health expenditure and household impoverishment due to out-of-pocket payments in healthcare were included. The review particularly looks at the incidence of CHE and impoverishment, defined, as the proportion of households whose OOP spending on healthcare is catastrophic or drives them into poverty. The intensity of $\mathrm{CHE}$ or impoverishment defined as the extent to which the household expenditure exceeds the set threshold or poverty line was also included. In addition, studies that assessed the risk factors associated with the observed levels of incidence in $\mathrm{CHE}$ are reviewed.

The review considered studies that assessed $\mathrm{CHE}$ and/or impoverishment due to seeking any type of healthcare service including HIV/AIDS, TB, chronic illnesses, Malaria and maternal healthcare services. The review was restricted to studies undertaken in any of the 45 SSA countries with coverage of either part of the country, the entire country or multiple countries. Articles were considered for inclusion if they were observational studies, cross-sectional studies, case-control, comparative or longitudinal studies. Articles that were opinion papers or general literature review on $\mathrm{CHE}$ or impoverishment, qualitative studies that discussed $\mathrm{CHE}$, those that addressed methodological issues and global macro analysis on CHE were excluded. These articles do not provide outcome measures that are relevant for this review such as the incidence or intensity of CHE.

\section{Search strategy}

This review commenced with a general search on Google Scholar; and then searches in several databases namely: PubMed, EBSCO [EconLit, Psycholnfo, CINAHL], Web of Science, and Jstor. Grey literature of relevant organizations virtual libraries such as WHO, and the World Bank were also searched. In addition, a forward search of authors mentioned in selected articles was also conducted. The search terms included: 'Catastrophic', 'Impoverishment', 'Financial burden', 'Economic burden', and under PubMed search the MESH terms for health expenditure, healthcare costs and SubSaharan African countries were included. These words were used for all the other database searches. The detailed search string for various databases is provided in Appendix 2-A. Only studies published in English language in the last 10 years (2006-May 2017) were included for review.

\section{Data extraction and analysis}

The main reviewer extracted and analysed data from all articles in consultation with the other reviewers. Information extracted from the publications included: context of the study (country and year of publication), characteristics of the included population, methodology (design of the study, data source, sample size, type of analysis), primary [incidence and intensity of $\mathrm{CHE} /$ Impoverishment) and secondary outcomes 
(determinants of $\mathrm{CHE}$ ). Studies were grouped by the outcome measure. As a primary outcome measure, the incidence and intensity of catastrophic expenditure, and impoverishment is used. To measure the impact of OOP on household expenditure; varying thresholds are applied which varied from $5 \%$ to $40 \%$ as a ratio of household expenditure or non-food expenditure. Information on the determinants of CHE and impoverishment were reported as secondary outcomes. Articles were also classified according to four major SSA regions (West Africa, East Africa, Southern Africa and Central Africa).

\subsubsection{Quality and risk of bias assessment}

Although quality assessments are not a standard requirement in scoping reviews, it has been argued that the lack of it could minimise the rigor, and challenge the interpretation of the findings (Levac et al., 2010). In light of this, a quality assessment of the studies was conducted by the main reviewer in consultation with the other reviewers. The Quality Assessment Tool for Observational Cohort and Cross-Sectional Studies (See Appendix 2-B) was applied to evaluate the quality of the studies included. The tool is recommended by the National Institutes of Health $(\mathrm{NIH})$ and has been used in several systematic reviews to assess internal validity (Wardle \& Steel, 2015). For each question studies are given scores on a Yes [1] and No [0] and others which include CD, cannot determine; NA, not applicable; NR, not reported.

All the studies included in this review were assessed for quality using the criteria that fits the respective studies, for those studies some elements of the criteria did not apply, these were marked as not applicable. The assessment of exposure measures was only done for those studies that focused on the risk factors associated with CHE.

\subsubsection{Study selection}

The initial search identified a total of 512 articles from the main journals and another 33 articles from the additional databases of WHO and the World Bank. Once duplicates were removed a total of 501 articles remained. Using title and abstracts, one reviewer screened all the identified articles based on an agreed inclusion criterion with the other two reviewers. A total of 445 articles were excluded largely due to being nonSSA specific, or for having a general focus on national health expenditure instead of $\mathrm{CHE}$ and impoverishment. A total of 56 articles remained that were fully assessed for eligibility, a second reviewer went through these selected articles and provided recommendations. The three reviewers had concurrence to include 34 articles in the final review analysis. The main reason for dropping 22 studies included the fact that the outcome was level of OOP and not the proportion that was catastrophic. Also, these articles do not provide information that allow us to calculate the proportion of OOP that is/was catastrophic for households, a global analysis of studies that included one or two SSA countries, discussion papers or general literature review that provide a general 
understanding of $\mathrm{CHE}$, qualitative studies that discussed $\mathrm{CHE}$, and methodological studies. Figure 2.1 represents the PRISMA flow chart for the studies selection process.

Figure 2.1: Flow chart of the studies filtered and assessed
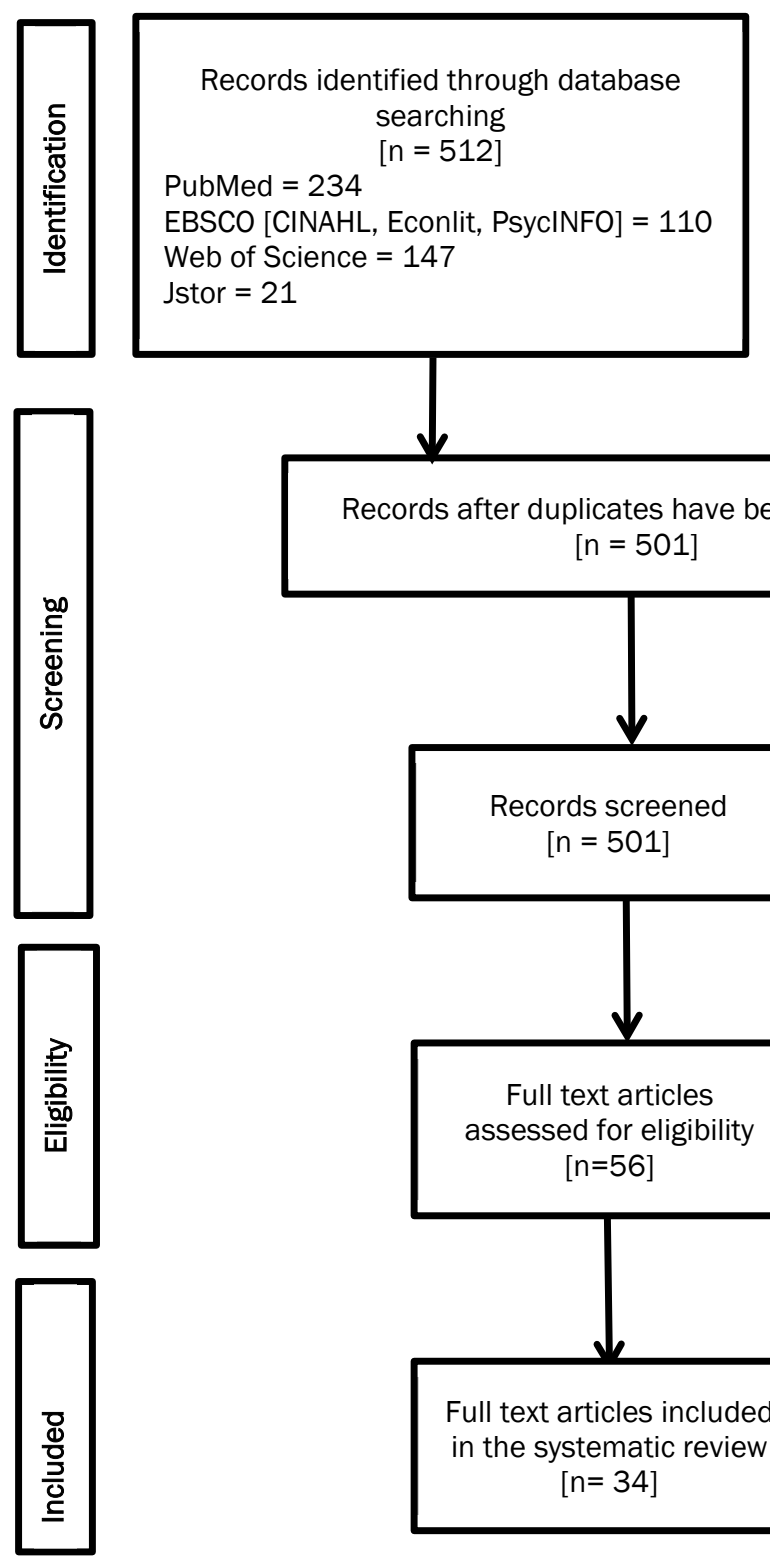

Records after duplicates have been removed $[n=501]$

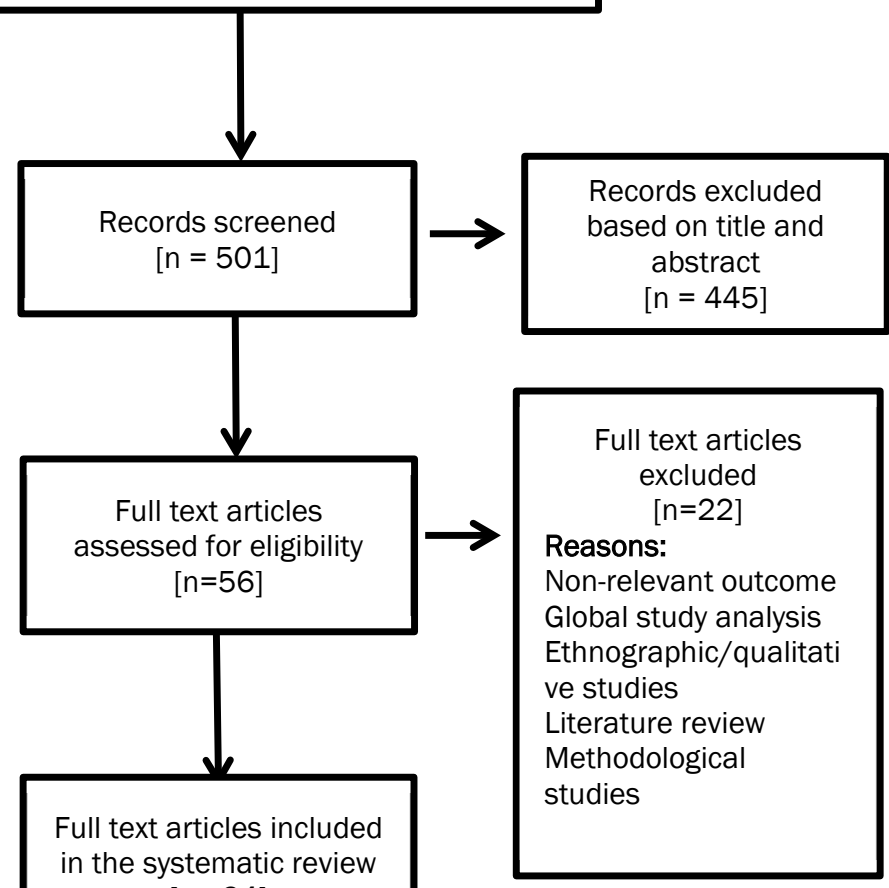




\subsection{Results}

\subsubsection{Characteristics of the included studies and quality of data}

On average, all the studies met the quality criteria, apart from 6 studies $(11,13$, $24,29,31,34$ ) that assessed exposure factors that vary by levels that did not examine the different levels of exposure. In addition, one multi-country study [18] did not report on the sample size thus the three criteria related to the study sample couldn't be determined. Figure 2.2 shows the ratio of studies that met the respective criteria.

Of the 34 studies assessed half were from the West Africa region [11 from Nigeria], eight from East Africa region [4 from Kenya], seven from South African region [2 from South Africa and 2 that were comparative of South Africa with Lesotho and Mozambique respectively], one from Central Africa region and, one covered three SSA regions [East, West and South Africa]. One could argue that literature from Central Africa region was missing because the region is largely francophone while the review focused only on English studies. However, there were several studies included from other francophone countries including DRC, Burkina Faso, Mali, Benin, Senegal, and Côte d'Ivoire. All the studies identified were observational, of which 27 were cross sectional studies, 3 were cross sectional comparative across countries in the regions, 2 were modelled longitudinal, one was a case control and another a prospective observational study.

Nineteen (19) of the studies focused on general healthcare, while 15 focused on diagnostic categories including 4 on chronic [non-communicable diseases], 5 on HIV/ART care and treatment [with one being comparison with Obstetric and TB], 2 on obstetric care [one being a comparison with TB, HIV/ART] and 3 on TB [One comparison with Obstetric and HIV/ART], and 3 on Malaria. The number of studies increased over years with only 8 (24\%) being published between 2006-2011, while 26 studies (76\%) were published between 2012 and May 2017. Half of the studies covered a sub-national population within the respective country, while 14 studies $(41 \%)$ had a national coverage, and 3 studies were multi-county in that they focused on more than one country. The national studies utilised data from various national household surveys including the National Living Standard, Social Economic Survey, Poverty Monitoring Survey, Health Expenditure and Utilization Survey while the sub-national studies sampled the respective regions or specific target population. A few other studies utilised hospital data to gather data on expenses paid for the various services provided, the limitation was the small sample sizes. (See appendix 2-C) for all studies included in the review by various characteristics. 


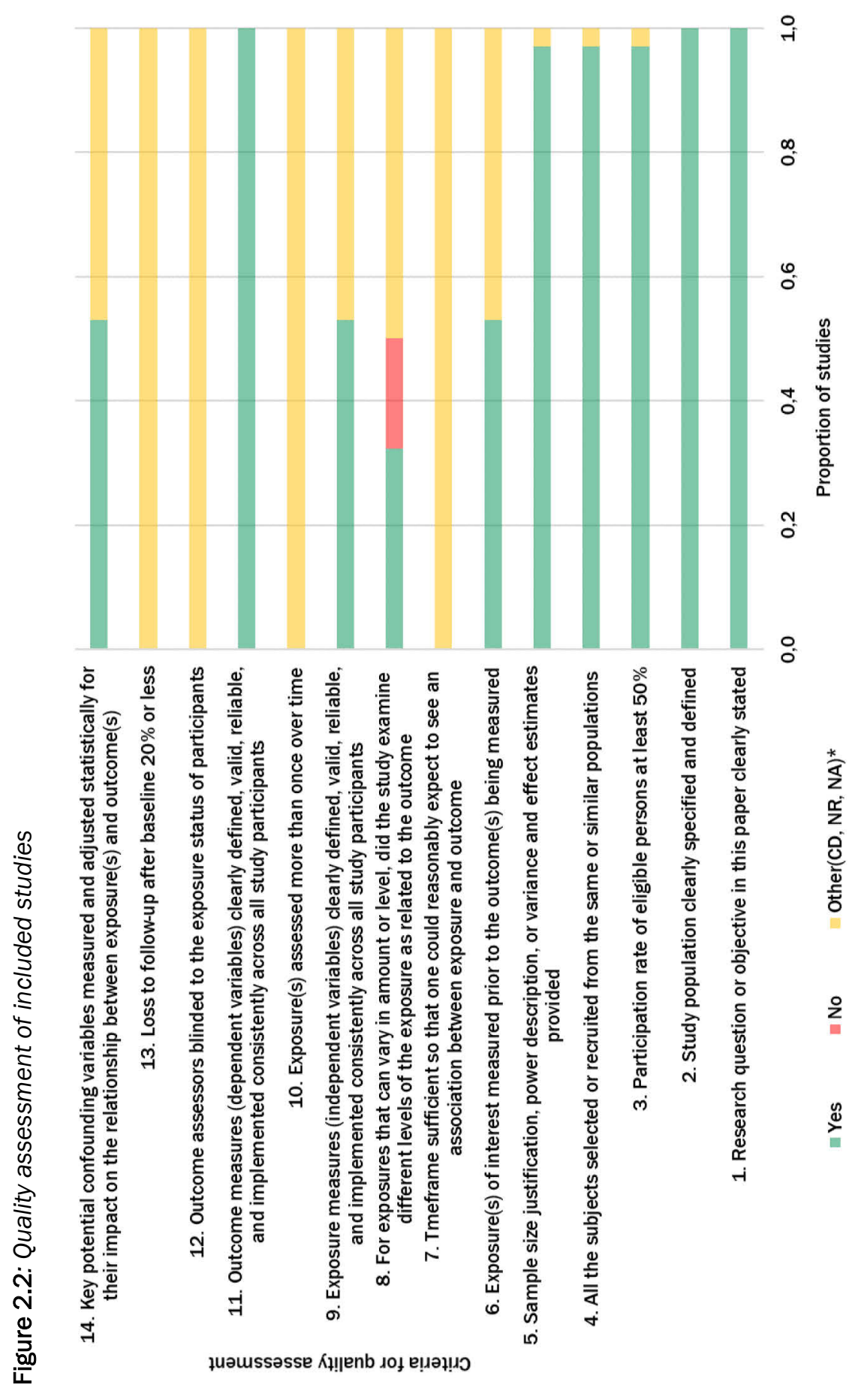

$\stackrel{\circ}{N}$ 


\subsubsection{Incidence and intensity of catastrophic health expenditure in SSA countries}

The large majority of studies focused only on the incidence of $\mathrm{CHE}(n=23)$, while some focused on both incidence and intensity $(n=11)$, and a set of others focused on the determinants of CHE $(n=18)$. CHE varied greatly between countries. However, crosscountry comparisons are difficult because of the different thresholds, sample sizes and data sets used in the various studies. Given these variations we shall discuss the magnitude and distribution of catastrophic payments based on the most commonly used thresholds, that is $10 \%$ of household income (Adam Wagstaff \& Doorslaer, 2003) and $40 \%$ of non-food expenditure (Xu et al., 2003a).

The proportion of households facing catastrophic payments varied widely by the threshold applied as shown in table 2.1. In most of the studies, the incidence of catastrophic expenditure was seen to be lower when higher thresholds were applied $(\mathrm{H}$. E. Ichoku et al., 2009; Mchenga et al., 2017), at 10\% threshold the average incidence of $\mathrm{CHE}$ was $23 \%$ while at $40 \%$ threshold the average was $17 \%$. Generally, we noted that $\mathrm{CHE}$ was highest when a specific diagnostic service was assessed. Amongst the various diagnostics HIV/ART and Malaria had the highest incidence with inpatient and outpatient variations. For example in Nigeria while CHE for outpatient HIV services was $7.7 \%$ and $40.3 \%$ at $40 \%$ threshold and $10 \%$ threshold respectively, the inpatient HIV patients had the highest incidence of $\mathrm{CHE}(100 \%)$ at $10 \%$ threshold household expenditure, while at $40 \%$ threshold the incidence was reduced to $94.3 \%$ (O. E. Onwujekwe et al., 2016). There was a high incidence of $\mathrm{CHE}$ at $40 \%$ non-food expenditure in the Democratic republic of Congo amongst hospitalised children with severe Malaria which was at $81.1 \%$ capacity to pay (Ilunga-llunga et al., 2015). Both studies with a high incidence targeted specific groups of patients and thus were not national representative surveys. TB patients also incurred a high incidence of $\mathrm{CHE}$ in Benin at $71.8 \%$ at the threshold of $10 \%$ of household expenditure (Laokri et al., 2014).

Variations are also observed within countries, for instance two national studies in Nigeria that focused on $\mathrm{CHE}$ at $40 \%$ of non-food expenditure, one reported $\mathrm{CHE}$ of $1.7 \%$ (H. Eme Ichoku \& Fonta, 2009), while the other (Ataguba, 2012) reported ten times more at $17.2 \%$. However, in the same studies, at $10 \%$ of household expenditure, $\mathrm{CHE}$ was closer in range at $22.7 \%$ and $25.7 \%$ respectively. The figure 2.3 below shows the variations in the incidence of $\mathrm{CHE}$ in various SSA countries, with many countries still experiencing high $\mathrm{CHE}$ over time. 


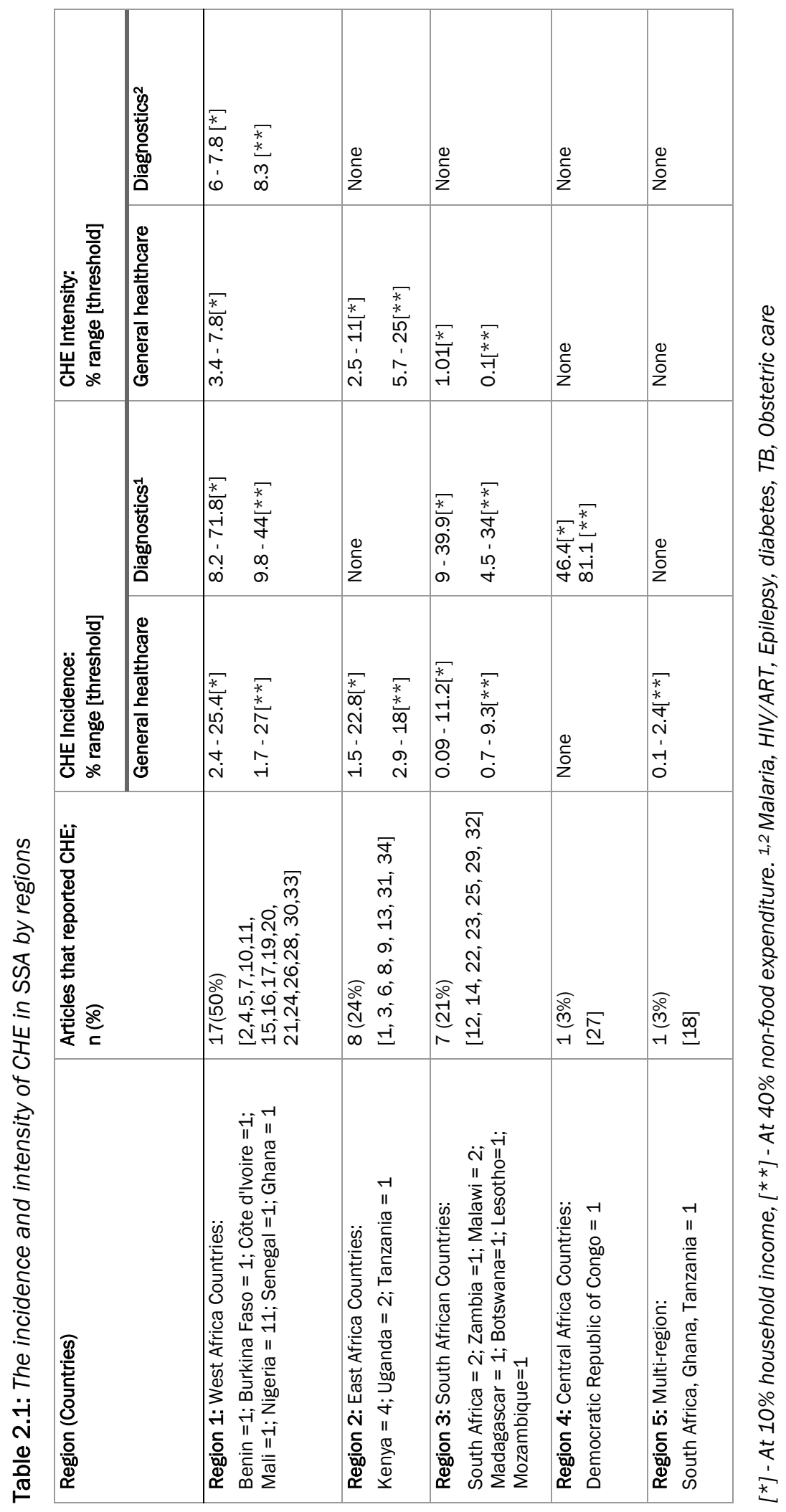




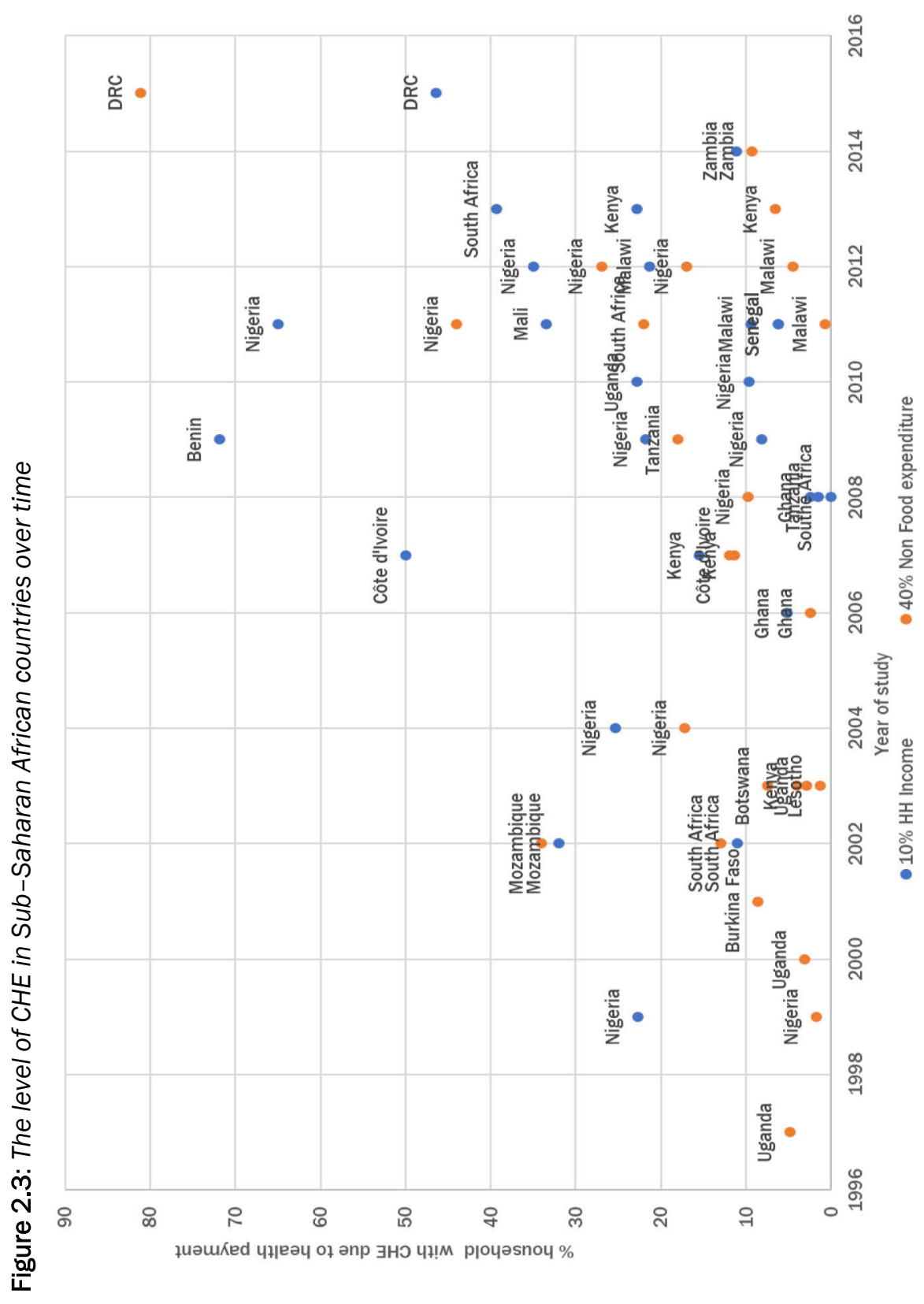

ล 
The intensity of catastrophic health expenditure, which is an indication of how much expenditure exceeds the thresholds ranged from $0.1 \%$ to $25 \%$ when the $40 \%$ proportion of non-food household expenditure threshold is applied, and $1 \%-11 \%$ when the $10 \%$ proportion of household expenditure is applied. There was relatively little difference between the intensity reported for general healthcare and that reported for specific diagnostics like HIV-ART, TB, Malaria and chronic illnesses. Most studies on diagnostic care did not report on intensity thus we have less evidence to discuss the severity of $\mathrm{CHE}$ due to the use of diagnostic services and treatment. The intensity of CHE was found to be lowest amongst South African countries while in East Africa and West Africa the intensity was within the same range.

\subsubsection{Determinants of catastrophic health expenditure}

Eighteen (18) studies assessed the determinants of $\mathrm{CHE}$ in the respective countries for both general healthcare and specific diagnoses. The articles assessed various determinants; thus, this review will discuss the overarching determinants reported in the majority of studies. These are summarised in Table 2.2. See Appendix 2$\mathrm{D}$ for determinants reported in each of the study.

\section{(i) Household economic/income status}

Households' income level is the most consistent determinant of CHE with higher income groups being less likely to incur $\mathrm{CHE}$ relative to middle income and lower income groups (Adisa, 2015; Ilunga-llunga et al., 2015; Su et al., 2006). This is also observed amongst HIV and Assisted Reproductive Techniques (ART) related studies. Lower income groups had a higher likelihood of incurring CHE on ART services, given that they are more likely to use their savings on food and other routine household expenditures (Dyer et al., 2013). Besides the type of disease, the power of association varied by country of study. For instance, amongst studies conducted on CHE due to tuberculosis (TB), it was found that the power of association (CHE -lower income groups) was higher in Benin (Laokri et al., 2014) than in Nigeria (Kingsley Nnanna Ukwaja et al., 2013).

\section{(ii) Type of healthcare provider}

In case healthcare services are provided by public hospitals, a higher CHE is observed. This is especially observed for inpatient services (Xu, Evans, et al., 2006; Xu, James, et al., 2006). Within the public healthcare system, seeking services at the primary healthcare level like health centres and posts had a reducing effect on CHE (Masiye et al., 2016; Séne \& Cissé, 2015). In some countries seeking care from a private health facility is associated with increased $\mathrm{CHE}$ relative to seeking care at public facility (Kingsley Nnanna Ukwaja et al., 2013), while in others seeking care from public and private is associated with higher CHE compared to confessional structures (Ilunga-Ilunga et al., 2015). Seeking services from traditional healers due to cultural beliefs on various illnesses is associated with high CHE (Brinda et al., 2014). 
Table 2.2: Determinants of Catastrophic Health Expenditure

\begin{tabular}{|c|c|c|c|c|}
\hline $\begin{array}{l}\text { Determinants of Catastrophic } \\
\text { Health Expenditure (CHE) }\end{array}$ & Western Africa & $\begin{array}{l}\text { South } \\
\text { Africa }\end{array}$ & $\begin{array}{l}\text { East \& Central } \\
\text { Africa }\end{array}$ & $\begin{array}{l}\text { Total studies } \\
\text { per } \\
\text { determinant }\end{array}$ \\
\hline $\begin{array}{l}\text { Household economic status: } \\
\text { Poor income households } \\
\text { Middle income households } \\
\text { High income households }\end{array}$ & $\begin{array}{l}4 \\
{[5,15,28,33]} \\
*\end{array}$ & $\begin{array}{l}2 \\
{[12,14]}\end{array}$ & $\begin{array}{l}4 \\
{\left[1,6,13,27^{*}\right]}\end{array}$ & 10 \\
\hline $\begin{array}{l}\text { Type of healthcare provider: } \\
\text { Private } \\
\text { Public }\end{array}$ & $\begin{array}{l}2 \\
{[11,24]}\end{array}$ & $\begin{array}{l}1 \\
{[14]}\end{array}$ & $\begin{array}{l}5 \\
{[1,9,13,27 *} \\
34]\end{array}$ & 8 \\
\hline $\begin{array}{l}\text { Type of illness: } \\
\text { HIV-ART } \\
\text { Tuberculosis } \\
\text { Obstetrics } \\
\text { Malaria } \\
\text { Chronic illnesses }\end{array}$ & $\begin{array}{l}5 \\
{[5,10,11,24,} \\
33]\end{array}$ & $\begin{array}{l}2 \\
{[12,29]}\end{array}$ & $\begin{array}{l}5 \\
{[1,6,9,13} \\
27 *]\end{array}$ & 12 \\
\hline $\begin{array}{l}\text { Household member } \\
\text { characteristics } \\
\text { Employment status } \\
\text { Education level } \\
\text { Gender/sex } \\
\text { Age of household members }\end{array}$ & $\begin{array}{l}5 \\
{[5,15,24,28,} \\
33]\end{array}$ & $\begin{array}{l}3 \\
{[12,22,} \\
29]\end{array}$ & $\begin{array}{l}7 \\
{[1,6,9,13} \\
27 *, 31,34]\end{array}$ & 15 \\
\hline $\begin{array}{l}\text { Geographical Location } \\
\text { Distance to the health facility } \\
\text { Residence (Rural/Urban) }\end{array}$ & $\begin{array}{l}3 \\
{[24,11,15]}\end{array}$ & $\begin{array}{l}3 \\
{[14,22,} \\
29]\end{array}$ & $\begin{array}{l}5 \\
{[1,6,9,31,34]}\end{array}$ & 11 \\
\hline $\begin{array}{l}\text { Social insurance/health scheme } \\
\text { Health insurance } \\
\text { Social network scheme }\end{array}$ & $\begin{array}{l}3 \\
{[5,11,28]}\end{array}$ & Nil & $\begin{array}{l}1 \\
{[9]}\end{array}$ & 4 \\
\hline $\begin{array}{l}\text { Household size and composition } \\
\text { Number of household members } \\
\text { Household with elderly people } \\
\text { Household with under } 5 \text { children }\end{array}$ & $\begin{array}{l}5 \\
{[5,10,11,24,} \\
33]\end{array}$ & $\begin{array}{l}2 \\
{[12,22]}\end{array}$ & $\begin{array}{l}6 \\
{[1,6,9,13,31,} \\
34]\end{array}$ & 13 \\
\hline
\end{tabular}

*Central Africa region (Democratic republic of Congo)

** numbers in the brackets shows the number of the study as per Appendix 2-D 


\section{(iii) Type of illness}

The presence of a household member with a chronic disease increases the likelihood of experiencing CHE (E. Barasa et al., 2017; Brinda et al., 2014; Su et al., 2006). While the number of illness episodes amongst adults significantly increases the odds of $\mathrm{CHE}$, the average number of illness episodes amongst children in a household has no effect on CHE (Su et al., 2006). Simple illness like coughs did not increase the risk of CHE (Buigut et al., 2015). In cases of TB care, households with HIV patient are more likely to incur CHE than those not affected by HIV (Kingsley Nnanna Ukwaja et al., 2013). Contrary to expectation, having a disability has no effect on CHE (Su et al., 2006). However, occurrences of adverse events such as accidents or injury increases the likelihood of CHE (Buigut et al., 2015; Séne \& Cissé, 2015).

\section{(iv) Characteristics of household members}

Characteristics of the household head and members were mentioned in the majority of studies. However, different studies focused on different parameters including age of the household member, employment status, education level and female-male headed households. Households with older heads and older main income earners, lower education or with unemployed heads are more likely to incur CHE (E. Barasa et al., 2017; Buigut et al., 2015). Full time employment is protective against CHE, especially amongst couples where the woman has a fulltime job (Dyer et al., 2013). Also, employment status and occupation are associated with $\mathrm{CHE}$, for instance having a household head who is a manual labourer increases the likelihood of CHE (Akinkugbe et al., 2012; E. Barasa et al., 2017; Brinda et al., 2014).

There are studies with different results for instance a study in Zambia shows that the education and employment status of the household head is not significantly associated with the likelihood of incurring CHE (Masiye et al., 2016). Also, a study in Nigeria finds counter intuitive evidence that more educated households are more likely to incur CHE than the less educated household (Adisa, 2015).

There are mixed results about the probability of incurring $\mathrm{CHE}$ and gender. Female headed households have a higher probability of facing CHE (Xu, Evans, et al., 2006). On the contrary, a study in Botswana observed that female headed households are less likely to incur CHE (Akinkugbe et al., 2012). In another study in Nigeria, households with a male patient are more likely to experience CHE (Kingsley Nnanna Ukwaja et al., 2013) whereas in another study in Côte d'Ivoire households of HIV infected women have a higher risk of incurring CHE (Beaulière et al., 2010).

\section{(v) Geographical Location and distance to health facility}

Location of residence is seen as an important predictor of CHE. However, this varies by the location of the study. In Kenya for instance households located in marginalized counties have higher odds of incurring CHE (E. Barasa et al., 2017), while in Benin a study on TB patients, shows the odds of CHE are higher for patients residing in urban areas but when confounded with education the effects disappear (Laokri et al., 
2014). A study in Nigeria conducted among patients at a rural hospital shows that urban residents incur higher rates of catastrophic payments, this is due to transportation costs to the rural hospital (Kingsley Nnanna Ukwaja et al., 2013). Generally, living in urban areas is protective of CHE (Arsenault et al., 2013; Cleary et al., 2013; Séne \& Cissé, 2015). However, it is found to be protective for non-poor, but not for the poor (Xu, Evans, et al., 2006). Living far from the nearest healthcare centre is associated with increased CHE (Arsenault et al., 2013; Masiye et al., 2016).

\section{(vi) Social insurance/welfare scheme}

Informal financing mechanisms through mutual organisations, informal groups and merry go rounds unlike formal health insurance is observed to reduce the risk of CHE (Adisa, 2015). Thus, patients with a poor social network are more likely to incur CHE (Laokri et al., 2014). Households that are enrolled in health insurance, are engaged in mutual health organizations or an informal social safety net (such as membership in a merry go round) have a reduced risk of catastrophic spending (Buigut et al., 2015; Séne \& Cissé, 2015).

In certain cases health insurance is not a significant determinant, for instance in Kenya because it only covers a small proportion of households and only inpatient services (Xu, James, et al., 2006). Health insurance is observed not to protect households from CHE due to HIV/ART services. A study in Côte d'lvoire observed no association between CHE and households having health insurance. This is because households continue to cope with HIV related costs for a long time thus the financial burden increases (Beaulière et al., 2010).

\section{(vii) Household size and composition}

Households size is associated with $\mathrm{CHE}$, with larger households (of more than 5 members) having a higher risk of incurring CHE (Akinkugbe et al., 2012; E. Barasa et al., 2017; Beaulière et al., 2010; Brinda et al., 2014). It is observed that having an elderly member (above 65 years) in the household imposes a higher risk of CHE for the household meaning that elderly people are more vulnerable (E. Barasa et al., 2017; llunga-llunga et al., 2015; Xu, Evans, et al., 2006). If the household has an elderly patient (older than 40 years) CHE is likely to be high(Laokri et al., 2014; Kingsley Nnanna Ukwaja et al., 2013). In Nigeria there seem to be a positive but not significant elderly effect (Adisa, 2015). Despite children being vulnerable to diseases, a study in Kenya showed that having a member aged under 5 decreased the odds of CHE (E. Barasa et al., 2017). 


\subsubsection{Household impoverishment in Sub Saharan Africa countries}

Household impoverishments due to $\mathrm{CHE}$ is measured using different poverty lines in the different studies including the subsistence poverty line, the national poverty line (NPL) and the international poverty line (IPL). A study in Uganda that used both the national [\$1.31] and the international [\$1.25] poverty line (Kwesiga et al., 2015), finds that the percentage poverty head count after health payment is higher when IPL is applied at $18.1 \%$ while that of the NPL was $17.1 \%$. Only one study in Malawi assessed impoverishment due to CHE for chronic illness (Q. Wang et al., 2016), all the other assessed impoverished due to $\mathrm{CHE}$ related to general healthcare. Figure 2.4 below summarises the level of impoverishment due to health payments by comparing the households impoverished after health payments with the poverty headcount before healthcare payments

Figure 2.4: Level of impoverishment due to health payment

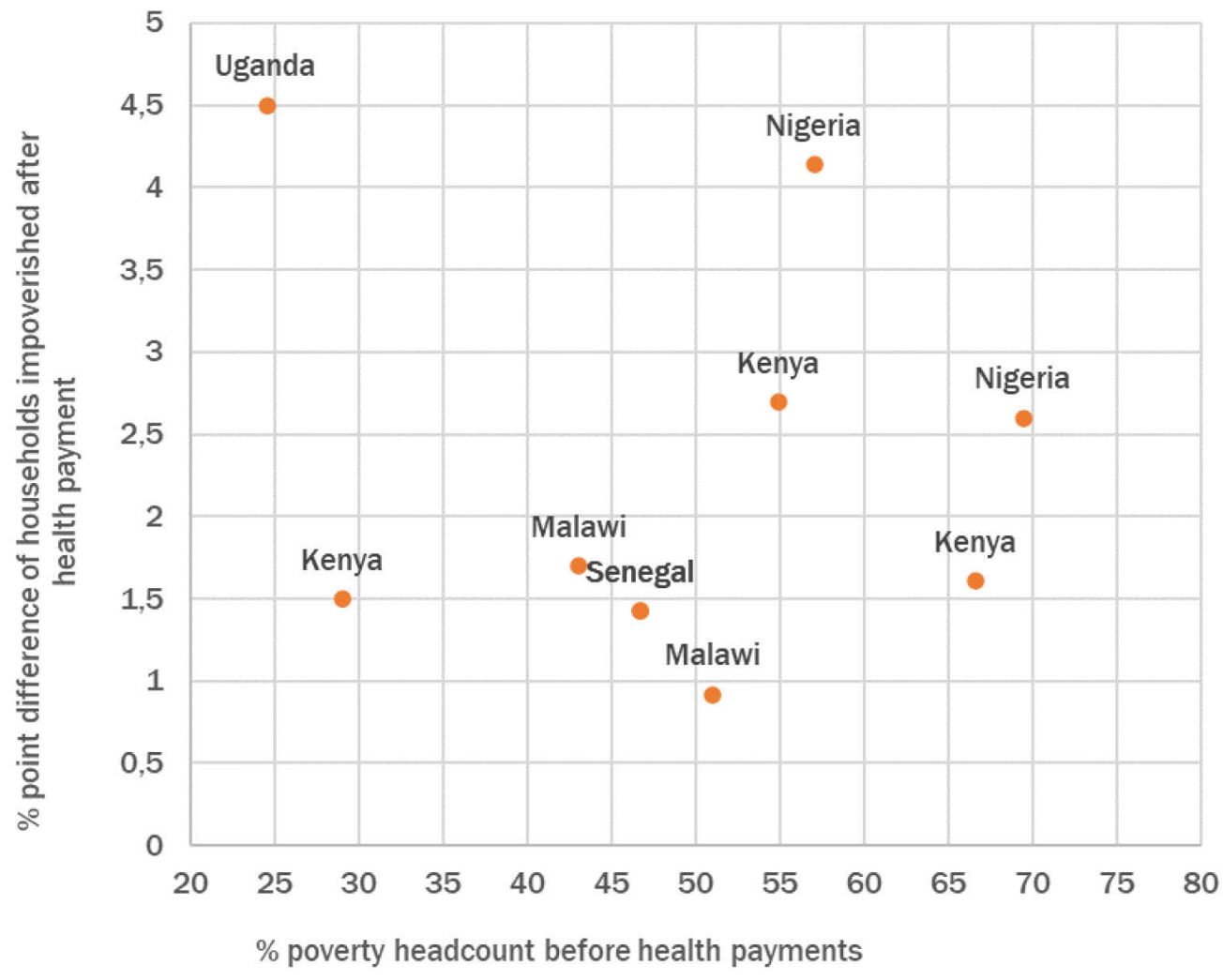


The percentage point difference of households that are impoverished ranged from $1.4 \%$ to $4.5 \%$ in the various countries. On average 2 percentage point of households are impoverished due to health payments across all countries with Nigeria and Uganda having the largest percentage point difference of household impoverished, $4.1 \%$ and $4.5 \%$ respectively. We note that the percentage point difference of household impoverished in some instances increased and also decreased over time across countries and also within the countries. This is, for example, the case of Kenya in 2003 the percentage point difference of households impoverished was 1.5 which increased to 2.7 in 2007 and decreased to 1.6 in 2013. In Nigeria, a study using data collected in 1999 showed 2.5 percentage point of households being impoverished and 4.1 in 2009 . In Malawi there was 0.9 percentage point households impoverished in 2011 and 1.7 in 2012. Out of pocket payments pushed a further $5.6 \%$ (ranging from $2 \%-7 \%$ ) of households on average into poverty with Uganda being the highest and an outlier at $18 \%$.

We observe no regional variations, but within regions, there are variations. For instance, in West Africa, a study in Nigeria (H. E. Ichoku et al., 2009) found 4.1 percentage point of households that were impoverished due to OOP relative to 1.4 percentage point of households observed in Senegal (Séne \& Cissé, 2015). In East Africa similar variations were observed, a study in Uganda showed a 4.5 percentage point difference of households impoverished (Kwesiga et al., 2015) compared to neighbouring Kenya with 2.7 (Chuma \& Maina, 2012) and 1.6 percentage point difference(E. W. Barasa et al., 2017) of households impoverished in 2007 and 2013 respectively. Table 2.3 below summarizes the pre-and post-poverty headcount after healthcare payment and the associated poverty incidence. 
Table 2.3: Impoverishment due to health payments in SSA by regions

\begin{tabular}{|l|l|l|l|l|l|}
\hline Author & Country & $\begin{array}{l}\text { Pre-payment } \\
\text { Poverty head } \\
\text { count(\%) }\end{array}$ & $\begin{array}{l}\text { Post-payment } \\
\text { Poverty head } \\
\text { count(\%) }\end{array}$ & $\begin{array}{l}\text { Households } \\
\text { Impoverished } \\
\text { (\%) }\end{array}$ & $\begin{array}{l}\text { Relative } \\
\text { Difference (\%) }\end{array}$ \\
\hline Xu et al., 2006 & Kenya & $29^{\alpha}$ & 30.5 & 1.5 & 5 \\
\hline $\begin{array}{l}\text { Kwesiga et al., } \\
2015\end{array}$ & Uganda & $24.5^{\beta}$ & 29 & 4.5 & 18 \\
\hline $\begin{array}{l}\text { Barasa et al., } \\
2017\end{array}$ & Kenya & $66.6^{\beta}$ & 68.21 & 1.61 & 2 \\
\hline $\begin{array}{l}\text { Ichoku and Fonta, } \\
2009\end{array}$ & Nigeria & $69.4 \beta$ & 72 & 2.6 & 4 \\
\hline $\begin{array}{l}\text { Chuma and } \\
\text { Maina, 2012 }\end{array}$ & Kenya & $54.9^{\beta}$ & 57.6 & 2.7 & 5 \\
\hline $\begin{array}{l}\text { Sene and Cisse, } \\
2015\end{array}$ & Senegal & $46.71^{\beta}$ & 48.14 & 1.43 & 3 \\
\hline $\begin{array}{l}\text { Mchenga et al., } \\
2017\end{array}$ & Malawi & $50.98^{\beta}$ & 51.9 & 0.92 & 2 \\
\hline $\begin{array}{l}\text { Ichoku et al., } \\
2009\end{array}$ & Nigeria & $57^{\beta}$ & 61 & 4.14 & 7 \\
\hline Wang et al., 2016 & Malawi & $43^{\varphi}$ & 44.7 & 1.7 & 4 \\
\hline
\end{tabular}

$\alpha$ Subsistence poverty line, $\beta$ National poverty line, $\phi$ International poverty line [\$1.25 per day]

\subsection{Discussion}

West African countries incurred higher $\mathrm{CHE}$ relative to the other regions. This could be because most studies utilized convenient sampling of pre-selected vulnerable groups with small sample sizes rather than national representative household economic surveys, which were largely used in other regions. Furthermore, it has been argued that the use of convenience sampling is likely to bias results and conclusions thus interpretation should be done with caution (Kankeu et al., 2013). For instance, of the 11 studies conducted in Nigeria only two (Ataguba, 2012; H. E. Ichoku et al., 2009) utilized national representative household surveys, and we note that most studies reported different incidences of $\mathrm{CHE}$.

Patients with HIV/ART, TB and Malaria experience the highest incidence of catastrophic expenditure. This could largely be due to the fact that individuals with HIV continue to incur health expenses throughout the time of their illness, while those with TB are in continuous medication for about 6 months or more, and Malaria could have several repeat episodes within a family.

Studies have found that affordability of treatment in LMICs is low as large proportions of population are pushed into poverty due to medicine procurement hence the need for subsidies (Niëns et al., 2010). However, this review revealed that nonmedical related costs like transportation costs which are invariably greater for the poor living far from the health facilities, food related costs, non-routine tests and inadequate care (due to shortages of drugs and medical services) in public primary healthcare 
facilities largely influence CHE which is consistent with other studies (Arsenault et al., 2013; Beaulière et al., 2010; O. Onwujekwe et al., 2009; Tomini et al., 2013). This therefore means that on the contrary, subsidizing the cost of drugs or removal of user fees alone may not necessarily protect households from CHE. It is revealing that nonmedical expenditures are much higher than medical expenditures, with food and transport being the two most significant expenditure components (O. E. Onwujekwe et al., 2016). We note that where user fees are abolished CHE declines for the non-poor but surprisingly remains the same for the poor, thus not encouraging the poor to seek care (Xu, Evans, et al., 2006).

All study findings are consistent that the poor have a higher incidence and are more likely to incur $\mathrm{CHE}$ than the well off. Furthermore, studies show that the poor are more burdened with OOP payments and CHE (Akazili et al., 2017; Chuma \& Maina, 2012; O. Onwujekwe, Dike, et al., 2010; O. Onwujekwe et al., 2012). This is largely due to the fact that for households with a low income even a small amount of healthcare costs can be catastrophic (Masiye et al., 2016). This is contrary to studies in LMICs elsewhere like Asia, whereby the well-off are seen to have a higher incidence of CHE given their likelihood to spend more on healthcare unlike the poor (Dorjdagva et al., 2016; Rashad \& Sharaf, 2015; van Doorslaer et al., 2007). This demonstrates that there is significantly less financial risk protection going to the poorest sections of the population in SSA.

Surprisingly seeking services from the public sector increases the risk of CHE, despite no or modest charges for public sector (Castillo-Riquelme et al., 2008). This is possibly because most people who seek services from public service providers are from lower income quintiles. In most SSA countries, people who seek care in the private sector are more likely to be well-off hence have the capacity to pay. Nevertheless, this is not the case in many other countries that show $\mathrm{CHE}$ to be higher amongst people seeking care in private hospitals. This could be another factor that may explain the relatively high incidence of $\mathrm{CHE}$ even where user fees have been removed, given the inadequate quality of services in public facilities(due to shortages of drugs and medical services), individuals are compelled to seek better care elsewhere (Masiye et al., 2016).

The review underscores the role of the type of illness in CHE. Consistent with other studies that have shown the impact of non-communicable chronic illnesses (Jaspers et al., 2015; Mondal et al., 2010), this review also notes that chronic illnesses contribute to a high risk of CHE (Counts \& Skordis-Worrall, 2016; Okoronkwo et al., 2015; Ughasoro et al., 2014). Putting into consideration that infectious disease like HIV, TB and Malaria are highly prevalent in Africa and have the highest incidence of CHE. This potentially poses a double burden on the households that are affected by both thus driving the incidence of $\mathrm{CHE}$ further up. We observe that the time on ART decreases the risk of CHE meaning that patients who can access continuous ART treatment can be more financially secure (Dyer et al., 2013). For HIV, when the main income earner is the one affected, time on HIV-ART increases the risk of CHE (Beaulière et al., 2010). Contrary to the notion of collaborative HIV/TB services we note that in case of TB care, households with HIV 
patient are more likely to incur CHE than those not affected by HIV because of the double disease burden (Kingsley N. Ukwaja et al., 2013).

Unlike in developed countries where health insurance is protective of $\mathrm{CHE}$, this review emphasises informal social networks and mutual organization common in the African setup, which help households to cope with costs. However, the review is inconclusive about the effect of formal health insurance in reducing CHE in SSA. There are nuances on the size of the household as a predictor of $\mathrm{CHE}$. Although a larger household size is associated with higher $\mathrm{CHE}$, households with more working adults are less likely to incur CHE (Buigut et al., 2015) perhaps supporting the economies of scale argument (Adisa, 2015). Furthermore it is also noted that smaller households have an increased risk of $\mathrm{CHE}$ which reflects a smaller support network from which financial assistance can be sort (Dyer et al., 2013). Elderly members in the household are seen to increase the risk of CHE (Séne \& Cissé, 2015) unlike children less than 5 years despite both being vulnerable to illnesses. This could be due to the fact that the elderly tends to also be income earners thus when ill there is dual burden unlike children who are under care of an elder. This is consistent with findings in other studies (Van Minh et al., 2013).

Distance to the health facility is associated with an increased likelihood of CHE, highlighting the significance of distance in increasing cost of access to healthcare (Masiye et al., 2016). Households in rural areas are also seen to experience higher CHE relative to those in urban areas excluding slum dwellers. Similar findings are observed in studies in Vietnam, Thailand and Serbia (Arsenijevic et al., 2013; Limwattananon et al., 2007; Van Minh et al., 2013).

We observe that women are more likely to incur CHE due to their low financial status (Adisa, 2015; Beaulière et al., 2010). In addition, we note that domestic violence against women increases the likelihood of experiencing CHE given women's welfare is vital to the household and injustice against them affects their income contribution, health and well-being (Brinda et al., 2014).

Contrary to the notion that health payments have a higher impact in countries where poverty is high (Murray et al., 2003), we observe variations in the level of impoverishments in relation to the poverty head count before health payments. For instance, in Uganda the level of poverty before healthcare payment was low, but the proportion of households impoverished as a result of health payment was higher than in all other countries. The proportion of households impoverished was seen to increase over time across the various countries, with the rapidly increasing population in Africa where the majority live below the poverty line more people could be pushed into poverty if the right financial risk protection measures are not put in place. It is inconclusive if impoverishment due to healthcare payments was permanent or transitory as no study in this review provided for that. An answer to this could however be given using panel data which are limited and unavailable at a national scale in SSA. 
We observed some limitations that should be considered when interpreting the findings presented in this chapter. First, the studies utilised different survey data including national household surveys, targeted population surveys and hospital data. Secondly, there were variations in the measurement of expenditure with some studies including only direct medical costs while others assessed both direct and indirect medical costs (E. Barasa et al., 2017). Also, there were variations of the thresholds applied across the different studies to measure $\mathrm{CHE}$, which makes it challenging to draw direct country comparisons. Furthermore the proportion of households that experience CHE is dependent on the threshold used to define it (Jaspers et al., 2015).

In addition, impoverishment was measured using different poverty lines including subsistence, national and international poverty line. Given the main aim was to focus on CHE studies the articles assessed on impoverishment were not exhaustive of the available literature on the same in SSA countries, but a representation of those that assessed both $\mathrm{CHE}$ and impoverishment.

We note that several studies rely on data collected several years (up to 10 years) back before the article was published thus not providing a true reflection of the current context. Furthermore, use of alternative data means that the data was not solely collected for the purpose of this type of analysis thus could bias the results.

The search and selection process were mainly conducted by the lead reviewer. This could lead to a limitation or bias in the information retrieved from the articles selected for final review. However, other two reviewers were involved in deciding the key search words and, the search string was discussed and agreed upon by the three reviewers. At every stage of the selection process, the three reviewers held frequent discussions to analyse the output(s). In addition, the use of MESH terms for key journal searches like PubMed ensured that all possible words were included in the search. We note that this has no substantial impact on the findings given the final articles that were reviewed represented 17 Sub-Saharan African countries from across all the different regions and a range of health areas.

\subsection{Conclusions}

Overall, the results show that $\mathrm{CHE}$ and impoverishment are pervasive across all SSA countries, and the magnitude varies across and within countries and over time. The factors that keep CHE higher, vary across the countries and are seen to cut across various socioeconomic and demographic characteristics including economic status, type of healthcare provider, type of disease, household size, geographical location, and social support schemes/network.

This review underscores the importance of studies that assess CHE in SSA, and we notice the increased interest in this area given the rise in number of studies over time. However, the majority of the studies were cross sectional thus not sufficient for over time analysis. 
Financial risk protection interventions in SSA have primarily focused on the supply side through subsidizing drugs, removal of user fees or provision of free healthcare, and most recently expansion of social insurance schemes. However, this review has shown that most of these do not necessarily protect households from $\mathrm{CHE}$ due to other related non-medical costs like transport and food.

The review emphasised on the role of informal social networks which are common in Africa like merry go round or mutual organisations, and hence the need to explore policy innovations through these social networks like insurance packages for informal or mutual groups. This review further highlights specific illnesses that drive $\mathrm{CHE}$. In light of this, it is paramount for SSA countries to consider comprehensive and integrated health financing policies that cut across diseases, as this could help to draw synergies and efficiencies across disease areas, and deal with possible dual disease burden. In addition, this review has paid specific attention to groups that are not financially autonomous. The fact that $\mathrm{CHE}$ was seen to be higher amongst the poor is an indication that the measures put in place have not been effective in protecting the poor. Given the context, there is a need to strengthen the financial risk protection interventions such that they are more holistic and effective in protecting the most vulnerable of population from catastrophic and impoverishing effects of health payments.

Further research in SSA would be more beneficial if panel data were utilised to facilitate continuous monitoring of trends over time. Given the variations in CHE across regions and socioeconomic groups, it is crucial to assess the existing inequalities and their drivers over time to inform targeted policy interventions. However, while CHE is a robust measure for monitoring financial risk protection, it is based on OOP payments hence excludes those who refrain from seeking care. To understand the impact of the lack of financial risk protection for the wider population, research needs to explore the extent of unmet need due to cost among those who forgo care due to cost barriers.

Additionally, even when households can afford healthcare services or have the ability to pay, they can be limited to access care due to economic setbacks triggered by unanticipated shocks. Furthermore, in low-income countries, shocks cause economic strain to households making it challenging to rebuild financial cushion. Therefore, understanding the interplay between shocks and the utilisation of healthcare services would be instrumental to policymakers in addressing the economic resilience of the households to achieve a more significant impact on the well-being of the population. 


\section{Chapter 3 :}

Decomposition of Changes in Socioeconomic Inequalities in Catastrophic Health Expenditure in Kenya

\section{Based upon:}

Njagi, P., Arsenijevic, J., \& Groot, W. (2020). Decomposition of changes in socioeconomic inequalities in catastrophic health expenditure in Kenya. PLOS ONE, 15(12), e0244428. https://doi.org/10.1371/journal.pone.0244428 


\section{Abstract}

Background: $\mathrm{CHE}$ is frequently used as an indicator of financial risk protection. $\mathrm{CHE}$ exists when health expenditure exceeds a certain threshold of household consumption. Although CHE is reported to have declined in Kenya, it is still unacceptably high and disproportionately affects the poor. This study examines the socioeconomic factors that contribute to inequalities in $\mathrm{CHE}$ as well as the change in these inequalities over time in Kenya.

Methods: We used data from the KHHEUS in 2007 and 2013. The concertation index was used to measure the socioeconomic inequalities in CHE. Using the Wagstaff (2003) approach, we decomposed the concentration index of $\mathrm{CHE}$ to assess the relative contribution of its determinants. We applied Oaxaca-type decomposition to assess the change in $\mathrm{CHE}$ inequalities over time and the factors that explain it.

Results: The findings show that while there was a decline in the incidence of $\mathrm{CHE}$, inequalities in CHE increased from -0.271 to -0.376 and was disproportionately concentrated amongst the less well-off. Higher wealth quintiles and employed household heads positively contributed to the inequalities in CHE, suggesting that they disadvantaged the poor. The rise in $\mathrm{CHE}$ inequalities over time was explained mainly by the changes in the elasticities of the household wealth status.

Conclusion: Inequalities in CHE are persistent in Kenya and are largely driven by the socioeconomic status of the households. This implies that the existing financial risk protection mechanisms have not been sufficient in cushioning the most vulnerable from the financial burden of healthcare payments. Understanding the factors that sustain inequalities in $\mathrm{CHE}$ is, therefore, paramount in shaping pro-poor interventions that not only protect the poor from financial hardship but also reduce overall socioeconomic inequalities. This underscores the fundamental need for a multi-sectoral approach to broadly address existing socioeconomic inequalities. 


\subsection{Introduction}

As reviewed in Chapter 2, healthcare systems' reliance on OOP payments can impose a financial burden on households, preventing some from seeking care and turning catastrophic for those who do (Xu et al., 2007). It is estimated that by 2010,808 million people in the world experienced catastrophic costs, and another 97 million(\$1.90-a-day poverty line) were impoverished due to healthcare payments (WHO $\&$ World Bank, 2017). This is more profound in developing countries where OOP payments are a dominant feature of financing healthcare (Mussa, 2016). CHE has been widely used as an indicator of the extent to which the healthcare system protects households from healthcare-related financial hardship (Raban et al., 2013). CHE refers to any expenditure on health that threatens a household's financial ability to maintain its subsistence needs, and this does not necessarily refer to high costs (Ezat Wan Puteh \& Almualm, 2017). Therefore, catastrophic health expenditure occurs when OOP payments exceed a certain threshold of household consumption (Adam Wagstaff \& Eozenou, 2014). The poorest households are most at risk, given that even a small amount of health spending can have catastrophic effects (Haghparast-Bidgoli et al., 2015). As evidenced in chapter 1 , other groups vulnerable to $\mathrm{CHE}$ include households with chronically ill members, older members, and/or children (WHO, 2005).

In Kenya, health financing includes OOP payments for several services at different levels of care (Chuma \& Okungu, 2011). These payments have been shown to be regressive (Munge \& Briggs, 2014). CHE is still prevalent in Kenya (Kimani \& Maina, 2016) in spite, of the abolition of user fees at some levels of healthcare, such as maternity care and services for children under five. Moreover, 7.1\% of Kenyans are reported to have incurred $\mathrm{CHE}$ in 2018 (Salari et al., 2019). Although Kenya has a National health insurance scheme, its coverage and package are limited. The NHIF has not been able to reach out to the majority of Kenyans households and individuals, especially the poor and those in the informal sector (Carrin et al., 2007; Luoma et al., 2010; Muiya \& Kamau, 2013). Furthermore, up until 2014, the NHIF package only covered inpatient services, and even the new insurance scheme is based on premiums under a contributory and voluntary mechanism; hence, the poor may not be able to pay (Kazungu \& Barasa, 2017).

Kenya is characterised by a high level of inequalities in comparison to its neighbouring East African counterparts, Uganda and Tanzania. These inequalities are manifest in different forms and sectors, including health, and are observed across regions, genders, and even specific segments of the population (Kiringai et al., 2016; SID, 2004). As discussed in the introductory chapter, the Kenya healthcare system has been criticised for various disparities including distribution of resources and inequitable access to quality healthcare services. Furthermore, inequalities in access to healthcare services also exist, with the poorest forgoing essential services due to financial burden (Ilinca et al., 2019). 
Several studies have assessed the magnitude of CHE in Kenya and, to a limited extent, the inequalities in CHE (E. Barasa et al., 2017; Chuma \& Maina, 2012). Nevertheless, these studies have not assessed the determinants of these inequalities and the change in inequalities over time. Evidence suggests that inequalities in CHE exist and are disproportionately concentrated amongst particular groups (Kien et al., 2016; Q. Wang et al., 2015). In essence, catastrophic payments are more severe than they appear when presented as plain fractions of the population, as this can mask the fact that the poor are more likely to exceed this threshold (O'Donnell et al., 2007).

This study aims to assess the factors that influence inequalities in CHE and the change in these inequalities over time, i.e., from (2007 to 2013). Applying decomposition approaches, we add to the existing literature on CHE in Kenya and the region in two ways. First, we provide empirical evidence on the underlying determinants of inequalities in CHE. Second, we assess the change in CHE inequalities over time and the associated factors. Furthermore, in addition to the magnitude of inequalities, policymakers and researchers' are also interested in understanding the potential causes of socioeconomic inequalities (Heckley et al., 2016). A trend analysis of the changes in inequalities over time may also reveal whether current inequalities are newly emerging or persistent problems (WHO, 2013). Hence, the findings may inform the formulation of policies and actions aimed at reducing the existing inequalities in Kenya and its regions.

\subsection{Methods}

\subsubsection{Data source and sample}

This study utilises data from two rounds (2007 and 2013) of the KHHES. These are nationally representative cross-sectional surveys implemented by the $\mathrm{MOH}$ in collaboration with the Kenya National Bureau of Statistics (KNBS). The surveys collect data on various aspects of health, including the following factors.

Health and household expenditure data: This includes outpatient costs and routine health expenses in the 4 weeks preceding the survey, inpatient costs in the last one year, food expenditure in the last one month (2007) and last seven days (2013), monthly household expenditure in the last one month, and annual household expenditure.

Utilisation of outpatient and inpatient services. This includes access to health insurance, individual reasons for not seeking care when ill, the type of provider where care was sought, the mode of payment for services received and funds sources.

Demographic and socioeconomic characteristics. This includes the wealth status of the household, number of household members, education level of household members, age of household members, the gender of household members, and employment status of the household head.

The two samples are drawn from the national master sampling frame, the National Sample Survey and Evaluation Program (NASSEP). A new sample is drawn in each year using a multi-stage stratified sampling design. The 2007 wave surveyed a total 
of 8,453 households from 737 clusters, of which 506 (68.7\%) were rural, and 231(31.3\%) were urban clusters. The 2013 wave surveyed a total of 33,675 households drawn from 1,347 selected clusters, of which 814 (60\%) were rural and 533 (40\%) were urban clusters. The difference in the sample sizes between the two rounds of the survey is because, the 2007 sample was representative at the national level, while 2013 was representative at both the national and sub-national (county) level to cater to the newly created sub-national structure of governance in Kenya as per the new constitution of 2010. Both samples provide population weights at the household level.

The sample data utilised in the analysis of this study is limited to those who sought care when ill, and the CHE is based on the OOP payments for healthcare services received. This includes 3,728 households drawn from 737 clusters in 2007 and 16,526 households drawn from 1,347 clusters in 2013. We use the adjusted survey weights to account for the different survey samples.

\section{Variables}

The main variable of interest(dependent) in this study is the incidence of CHE, which is a measure of the OOP payment relative to the household capacity to pay (Xu et al., 2003a). The commonly documented drivers of CHE are used as the independent variables (Y. Li et al., 2012; Saksena et al., 2010). These drivers are also documented in Chapter 2 and they include the following:

(i) Demographic characteristics of the household (gender of the household head, age group of the household head, households with children under five years, households with elderly members, household size).

(ii) Socioeconomic characteristics of the households (wealth status, education level of the household head, employment status of the household head).

(iii) Geographical characteristic of the household (urban/rural residence)

(iv) Healthcare access factors (households with a chronically ill member, health insurance status, type of health provider).

\subsubsection{Measuring the incidence of catastrophic health expenditure}

This study calculated CHE using the approach proposed by XU (Xu, 2005). Xu estimates the incidence of catastrophic payments from the reported OOP payments as a share of $40 \%$ total non-food expenditure, also known as capacity to pay (CTP) (Xu et al., 2003a). This approach has been proposed by WHO and defines CTP as income remaining after subsistence needs have been met (WHO, 2005). To estimate CHE using this approach, we use data on OOP payments and household consumption expenditure, including food and non-food expenditure. OOP payments include both medical costs for inpatients and outpatients, such as consultation, diagnosis, drugs and admission costs, and non-medical costs for inpatient and outpatients such as transport. OOP payments are net of costs not paid directly by the patients, including those paid through insurance, exemptions, and reimbursements. 
Household (consumption) expenditure was calculated using the expenditure on food and non-food items. Food expenditure refers to how much the household spent on food and beverages items, excluding alcoholic beverages and food consumed outside the house. Non-food expenditure refers to recurring monthly expenditure such as rent and utilities, and annual expenditure such as house maintenance and education/fees, and capital expenditure, such as the purchase of assets.

To standardise and convert the costs into a common reference period of a year(annual), we annualised the outpatient costs by multiplying the total costs by 13 , given the costs relate to the last four weeks preceding the survey. This is also consistent with other studies in Kenya (E. Barasa et al., 2017; Kimani \& Maina, 2016; Salari et al., 2019).

We generated equivalised food expenditure by dividing each household's food expenditure by the equivalised household size. Equivalised household size is used to reflect household composition and size based on a scale of 0.56 (Xu et al., 2003a; Xu, Evans, et al., 2006). We identified the food expenditure shares of total household expenditure that are at the 45th (food45) and 55th (food55) percentiles across the whole sample. We then obtained the weighted average of food expenditure in the 45th to 55th percentile range. This gave the subsistence expenditure per (equivalent) capita, which is also the poverty line $(\mathrm{pl})$.

$$
\mathrm{pl}=\frac{\sum \mathrm{w}_{\mathrm{h}} * \mathrm{eqfood}_{\mathrm{h}}}{\sum \mathrm{w}_{\mathrm{h}}}
$$

Where, $w_{h}$ is the equivalised household size in the $45^{\text {th }}$ and $55^{\text {th }}$ percentile. The subsistence expenditure for each household $\left(\mathrm{se}_{h}\right)$ was derived by the following formula:

$$
\mathrm{se}_{\mathrm{h}}=\mathrm{pl} * \text { eqsize }_{\mathrm{h}}
$$

Household CTP, which is the household non-subsistence spending, was calculated using the following formula:

$$
\mathrm{CTP}=\left\{\begin{array}{c}
\exp _{h}-\mathrm{se} \\
\exp _{h}-\text { food }_{h} \text { if } \mathrm{se}_{\mathrm{h}} \leq \text { food }_{h} \\
\mathrm{se}_{\mathrm{h}}>\text { food }_{h}
\end{array}\right.
$$

Where, $\exp _{h}$ is the household expenditure, and $\operatorname{food}_{h}$ is the food expenditure. CHE binary variable was then created based on the fraction of OOP divided by CTP $(O O P / C T P)$, in which CHE took the value 1 if the fraction was greater or equal to 0.4 and 0 if otherwise.

\subsubsection{Measuring inequality in catastrophic health expenditure}

The concentration index $(\mathrm{Cl})$ is used to measure the extent of socioeconomicrelated inequalities in $\mathrm{CHE}$ for 2007 and 2013. The $\mathrm{Cl}$ has been extensively applied to quantify the extent of socioeconomic-related inequalities in health variables (G. Kjellsson \& Gerdtham, 2014). The $\mathrm{Cl}$ measures the extent to which the health variable differs 
across individuals ranked by the socioeconomic indicator (Kakwani et al., 1997). There are debates on the most suitable concentration index approach, but there is no consensus on which concentration index is 'superior' to the others (Erreygers, 2009a, 2009b; Adam Wagstaff, 2009). However, there is an emphasis that a suitable index should satisfy specific fundamental properties including, i) cardinal invariance: A linear transformation of the health variable does not affect the index value, ii) transfer: A small transfer of the health variable from a richer to a poorer individual translates into a propoor change in the concentration index, and iii) mirror: the Cls of the presence of the health variable and absence of the health variable should be mirror images of each other (Gustav Kjellsson \& Gerdtham, 2013).

The concentration index depends on the relationship between the health variable and the rank of the socioeconomic variable (O'Donnell et al., 2007). We classified the households into socioeconomic quintiles using per capita consumption expenditure to create a variable that ranks the households by their consumption (expenditure) status from the poorest to the richest quintiles. The concentration index was derived as follows:

$$
\begin{gathered}
\mathrm{CI}=\frac{2}{\mu_{\mathrm{h}}} \sum_{\mathrm{i}=1}^{\mathrm{n}}\left(\mathrm{h}_{\mathrm{i}}-\mu_{\mathrm{h}}\right)\left(\mathcal{R}_{\mathrm{i}}-\frac{1}{2}\right) \\
=\frac{2}{\mu_{\mathrm{h}}} \operatorname{cov}(\mathrm{h}, \mathcal{R})
\end{gathered}
$$

where $n$ denotes the number of observations, $h_{i}$ is the health variable $(\mathrm{CHE}), \mu$ is the mean of $\mathrm{h}$, and $\mathrm{R}_{i}-\frac{1}{2}$ is the fractional socioeconomic rank ranging from the poorest to the richest.

The concentration index ranges between -1 to +1 , with zero( 0$)$ value meaning no socioeconomic-related inequality. The concentration index is intended to show the direction of the relationship between $\mathrm{CHE}$ and the socioeconomic variable, and the degree of variability in the distribution of CHE (O'Donnell et al., 2007).

\subsubsection{Decomposition of the concentration index}

To assess the relative contributions of each factor to inequalities in $\mathrm{CHE}$, we decomposed the concentration index of $\mathrm{CHE}$ into its contributory factors using the Wagstaff approach (A. Wagstaff et al., 2003). This is the dominant decomposition procedure that has been comprehensively applied to explore the determinants of the socioeconomic gradient (Heckley et al., 2016; Kunna et al., 2017).

First, the concentration index of $\mathrm{CHE}$ was calculated, then that of each of the contributory factors. Second, we calculated the absolute contribution of each factor $(\chi)$ to the concentration index of $\mathrm{CHE}(\mathrm{y})$. A linear regression model is assumed to link the health variable $\mathrm{CHE}(\mathrm{y})$ to a set of factors $\left(\mathrm{c}_{k}\right)$ as follows:

$$
\mathrm{y}=\alpha+\sum_{k} \beta_{k} x_{k}+\varepsilon
$$


Given the relationship between y and $c$ in equation (5), the concentration index for $y$, can be written as:

$$
\mathrm{C}=\sum_{\mathrm{\kappa}}\left(\frac{\beta_{\mathrm{\kappa}} \bar{x}_{\mathrm{\kappa}}}{\mu}\right) \mathrm{C}_{\mathrm{\kappa}}+\frac{G C_{\varepsilon}}{\mu}
$$

where $\mu$ is the mean of $y, \bar{X}_{k}$ is the mean of $c_{k}$ ( $k$ set of factors), $c_{k}$ is the concentration index for $\mathrm{C}_{\mathrm{k}}$ (defined analogously to $\mathrm{C}$ ), and $\mathrm{GC}_{\varepsilon}$ is the generalised concentration index for the error term $(\varepsilon) .\left(\frac{B_{\kappa} \bar{x}_{\kappa}}{\mu}\right)$ is the elasticity indicating the impact of each factor on the outcome(y) (O'Donnell et al., 2007).

The results are intended to show the $\mathrm{Cl}$ for each of the explanatory variable, the elasticities, the absolute and percentage contribution of each variable. A negative concentration index indicates that the variable had pro-poor distribution, while a positive concentration index indicates the variable had a pro-rich distribution. The absolute contribution refers to the contribution of the explanatory variables to the overall $\mathrm{CHE}$ inequalities. The absolute contribution is the product of the elasticity and the partial concentration index of each of the explanatory variables. A positive absolute contribution shows that the variable favoured the worse-off, whereas a negative absolute contribution shows that the variable favoured the better-off.

\section{Oaxaca-type decomposition of change in concentration index}

Oaxaca-type decomposition (O'Donnell et al., 2007) is applied to estimate the change in CHE inequalities between 2007 and 2013.

$$
\Delta \mathrm{C}=\sum_{\kappa} \eta_{\kappa t}\left(\mathrm{C}_{\kappa t}-\mathrm{C}_{\kappa \mathrm{kt}-1}\right)+\sum_{\kappa} \mathrm{C}_{\kappa t-1}\left(\eta_{\kappa t}-\eta_{\kappa t-1}\right)+\Delta\left(\frac{\mathrm{GC}_{\varepsilon t}}{\mu_{\mathrm{t}}}\right)
$$

where $t$ refers to time period and $\Delta$ denotes first differences.

In equation (7), we weighted the difference in concentration indices $\left(C_{k t}-C_{\kappa t-1}\right)$ by the second-period elasticity $\eta_{\kappa t}$ and weighted the difference in elasticities $\left(\eta_{\kappa t}-\eta_{\kappa t-1}\right)$ by the first-period concentration index $\mathrm{C}_{\mathrm{kt}-1}$.

An alternative to equation (7) would be to weight the difference in concentration indices $\left(\mathrm{C}_{\kappa t}-\mathrm{C}_{\kappa \mathrm{k}-1}\right)$ by the first-period elasticity $\eta_{\kappa t-1}$ and weight the difference in elasticities $\left(\eta_{\kappa t}-\eta_{\kappa t-1}\right)$ by the second-period concentration index $C_{\kappa t}$ as expressed in equation (8) (O’Donnell et al., 2007).

$$
\Delta \mathrm{C}=\sum_{\kappa} \eta_{\kappa t-1}\left(\mathrm{C}_{\kappa \mathrm{t}}-\mathrm{C}_{\kappa \mathrm{kt}-1}\right)+\sum_{\kappa} \mathrm{C}_{\kappa \mathrm{t}}\left(\eta_{\kappa \mathrm{t}}-\eta_{\kappa \mathrm{t}-1}\right)+\Delta\left(\frac{\mathrm{GC}_{\varepsilon t}}{\mu_{\mathrm{t}}}\right)
$$

This explains the amount of change in $\mathrm{CHE}$ inequalities that was due to variations in changes in the unequal distribution of determinants $(\Delta C)$ or the elasticities of determinants $(\Delta \eta) . \Delta \mathrm{C} . \eta_{\mathrm{kt}}$ and $\Delta \mathrm{C} . \eta_{\mathrm{kt}-1}$ shows the changes in the amount of inequalities in the determinants, whereas $\Delta \eta$. $C_{k t-1}$ and $\Delta \eta$. $C_{k t}$ shows the changes in elasticities of the determinants. A positive sign means that the variable contributed to an increase in inequality, whereas a negative sign shows that the variable reduced the inequality over time.

All analyses were conducted in STATA 14/SE. 


\section{Results}

\subsubsection{Descriptive analysis of households experiencing catastrophic health expenditure}

First, we computed the incidence of $\mathrm{CHE}$, which showed a decrease from $11.4 \%$ in 2007 to $6.5 \%$ in 2013 at a threshold of $40 \%$ CTP. Similarly, we noted a decline in CHE across all the wealth status quintiles between 2007 and 2013. In absolute terms, the decline was greater among the lower wealth status groups; however, as percentage change, the richest group experienced a higher decline in $\mathrm{CHE}$ relative to other wealth categories. These analyses are presented in the Appendix 3-A. Figure 3.1 presents the overall incidence of $\mathrm{CHE}$ and the distribution of $\mathrm{CHE}$ across the five wealth quintiles in 2007 and 2013.

Figure 3.1: Incidence of Catastrophic Health Expenditure by Wealth Quintiles, 2007 and 2013

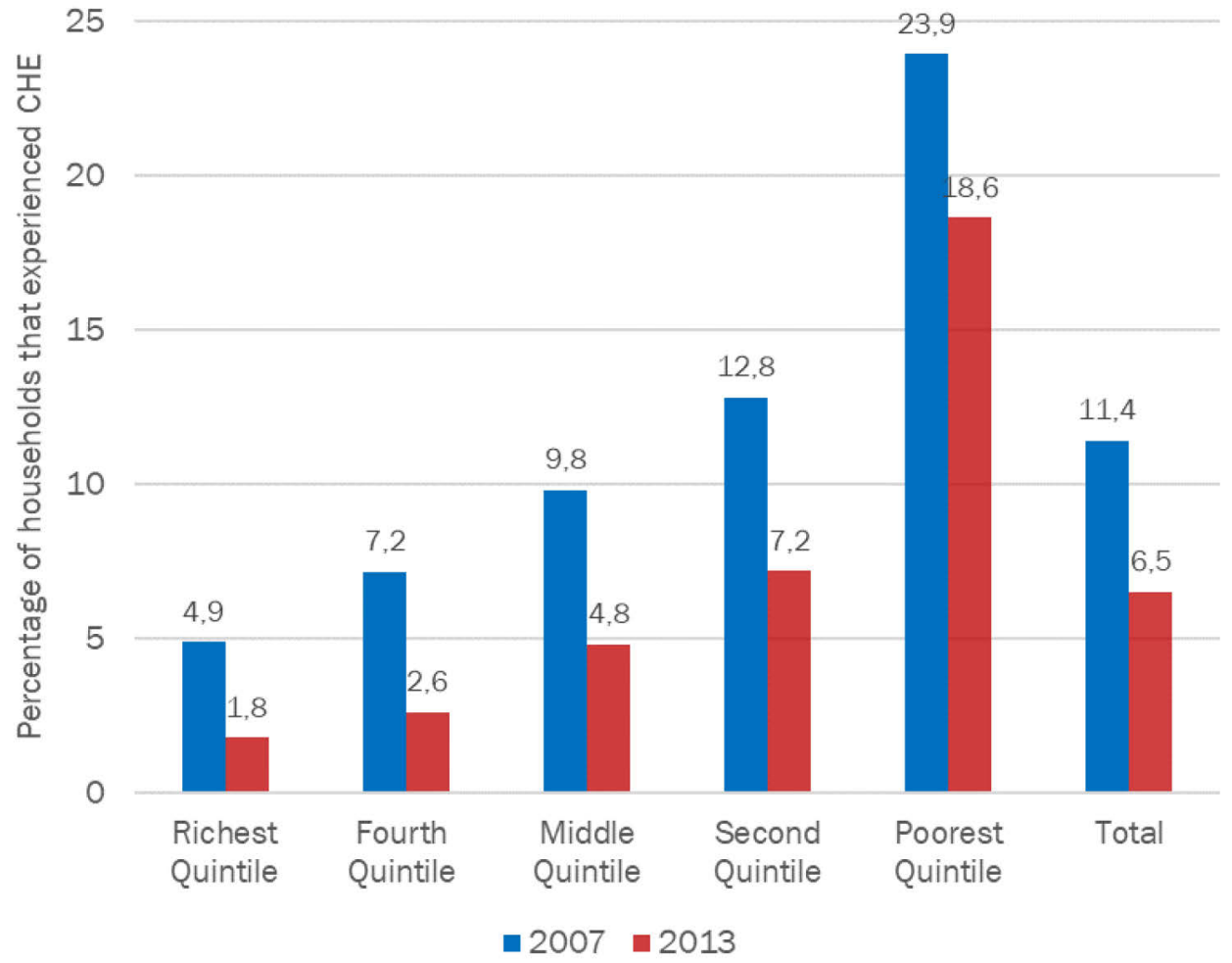


The distribution of households facing $\mathrm{CHE}$ by the various socioeconomic characteristics in 2007 and 2013 is presented in Table 3.1. The analysis shows an increase in the percentage of households facing $\mathrm{CHE}$ among the lower wealth quintiles and a decline in households facing $\mathrm{CHE}$ among the higher wealth quintiles. For instance, the poorest quintile increased from $26.5 \%$ to $34.3 \%$, while the richest quintile decreased from $15.7 \%$ to $9.2 \%$ in 2007 and 2013 respectively. The majority of the households that experienced CHE were female headed, had a less educated (primary level and below) household head, had an employed household head, and the household head was above 40 years. We also observed that the majority of the households that experienced $\mathrm{CHE}$ had children under five years, an elderly member, were not insured, lived in rural areas, and had a chronically ill member. However, we also observed that in the 2007 sample, the majority of households that experienced CHE were from small-sized households, whereas in 2013, the majority were from medium-sized households. The results further suggest that the majority of households that experienced CHE in 2007 had sought care from a public health provider, whereas in 2013 , the majority who experienced CHE had sought care from a private provider.

\subsubsection{Determinants of catastrophic health expenditure}

First, we fitted a logistic regression model to examine the factors associated with CHE in 2007 and 2013. The results are presented in the Appendix 3-B. The results show that, the determinants of facing CHE were similar but there were more significant factors in 2013 as compared to 2007.

In 2007, richer households (OR=0.10; 95\% Cl, 0.05-0.20), employed households' heads $(\mathrm{OR}=0.60 ; 95 \% \mathrm{Cl}, 0.445-0.808)$, and female-headed households $(\mathrm{OR}=0.65 ; 95 \%$ $\mathrm{Cl}, 0.47-0.91$ ) lowered the odds of incurring $\mathrm{CHE}$, whereas seeking care from private providers $(\mathrm{OR}=1.69 ; 95 \% \mathrm{Cl}, 1.26-2.27)$ and households with a chronically ill member $(\mathrm{OR}=1.37 ; 95 \% \mathrm{Cl}, 1.02-1.89)$ increased the odds of incurring $\mathrm{CHE}$.

The results further show that in 2013, richer households (OR=0.02; 95\% Cl, 0.010.05), employed household heads (OR=0.70; 95\% Cl, 0.51-0.96), medium-sized households $(\mathrm{OR}=0.50 ; 95 \% \mathrm{Cl}, 0.35-0.73)$, and larger households $(\mathrm{OR}=0.43 ; 95 \% \mathrm{Cl}$, 0.24-0.75) lowered the odds of incurring CHE. Meanwhile, seeking care from private providers $(\mathrm{OR}=2.90 ; 95 \% \mathrm{Cl}, 2.13-3.95)$, households with an elderly member(OR=1.58; $95 \% \mathrm{Cl}, 0.19-2.30)$, insured households ( $\mathrm{OR}=2.55 ; 95 \% \mathrm{Cl}, 1.73-3.77$ ), and households with a chronically ill member $(\mathrm{OR}=1.93 ; 95 \% \mathrm{Cl}, 1.45-2.57)$ increased the odds of incurring $\mathrm{CHE}$. 
Table 3.1: Socioeconomic Characteristics of the Households that Experienced Catastrophic Health Expenditure

\begin{tabular}{|c|c|c|c|c|c|}
\hline \multirow[b]{2}{*}{ Variables } & \multirow[b]{2}{*}{ Categories } & \multicolumn{2}{|l|}{2007} & \multicolumn{2}{|c|}{2013} \\
\hline & & $\begin{array}{l}\mathrm{N} \\
(427)\end{array}$ & $\begin{array}{l}\text { Percent } \\
(\%)\end{array}$ & $\begin{array}{l}N \\
(1084)\end{array}$ & $\begin{array}{l}\text { Percent } \\
\text { (\%) }\end{array}$ \\
\hline \multirow[t]{5}{*}{ Wealth status } & Poorest quintile & 162 & 26.5 & 547 & 34.3 \\
\hline & Second quintile & 99 & 18.5 & 225 & 21.7 \\
\hline & Middle quintile & 74 & 16.9 & 152 & 18.7 \\
\hline & Fourth quintile & 55 & 22.5 & 92 & 16.2 \\
\hline & Richest quintile & 37 & 15.7 & 68 & 9.2 \\
\hline \multirow{2}{*}{$\begin{array}{l}\text { Gender of household } \\
\text { head* }\end{array}$} & Female head & 288 & 70.9 & 729 & 67.2 \\
\hline & Male head & 118 & 29.1 & 355 & 32.7 \\
\hline \multirow{3}{*}{$\begin{array}{l}\text { Age group of } \\
\text { households' head* }\end{array}$} & Below 25 years & 20 & 4.9 & 41 & 3.8 \\
\hline & 25-40 years & 156 & 38.3 & 372 & 34.3 \\
\hline & $40+$ years & 231 & 56.8 & 671 & 61.9 \\
\hline \multirow{4}{*}{$\begin{array}{l}\text { Education of household } \\
\text { head* }\end{array}$} & No Education & 150 & 36.9 & 11 & 1.0 \\
\hline & Primary education & 159 & 39.1 & 805 & 74.4 \\
\hline & Secondary education & 82 & 20.2 & 178 & 16.4 \\
\hline & Tertiary education & 16 & 3.9 & 88 & 8.1 \\
\hline \multirow{2}{*}{$\begin{array}{l}\text { Employment status of } \\
\text { household head* }\end{array}$} & Unemployed HH head & 169 & 41.5 & 258 & 23.8 \\
\hline & Employed $\mathrm{HH}$ head & 238 & 58.5 & 826 & 76.2 \\
\hline \multirow[t]{3}{*}{ Household Size } & 1-3 Small household & 219 & 51.3 & 356 & 32.8 \\
\hline & 4-6 Medium household & 134 & 31.4 & 502 & 46.3 \\
\hline & $7+$ Large household & 74 & 17.3 & 226 & 20.8 \\
\hline \multirow{2}{*}{$\begin{array}{l}\text { Household with U5 } \\
\text { Children }\end{array}$} & Above 5 years & 287 & 67.2 & 573 & 52.9 \\
\hline & Below 5 years & 140 & 32.8 & 511 & 47.1 \\
\hline \multirow[t]{2}{*}{ Elderly (Above 60+) } & Below 60 years & 294 & 68.9 & 687 & 63.4 \\
\hline & Above 60 years & 133 & 31.2 & 397 & 36.6 \\
\hline \multirow[t]{2}{*}{ Health insurance } & Insured household & 353 & 82.7 & 803 & 74.2 \\
\hline & Not insured & 74 & 17.3 & 281 & 25.9 \\
\hline \multirow[t]{2}{*}{ Residence } & Rural residence & 331 & 77.5 & 752 & 69.4 \\
\hline & Urban residence & 96 & 22.5 & 332 & 30.6 \\
\hline \multirow{2}{*}{$\begin{array}{l}\text { HH with member with } \\
\text { Chronic illness }\end{array}$} & No chronic illness & 291 & 68.3 & 550 & 50.7 \\
\hline & Chronic illness & 135 & 31.7 & 534 & 49.1 \\
\hline \multirow{3}{*}{ Type of health provider* } & Public provider & 192 & 45.4 & 437 & 40.4 \\
\hline & Private provider & 151 & 35.6 & 491 & 45.4 \\
\hline & Other providers & 80 & 18.9 & 154 & 14.2 \\
\hline
\end{tabular}

*Dropped missing cases (<20) in 2007 


\subsubsection{Inequalities in catastrophic health expenditure}

Table 3.2 presents analysis of the concentration indices for CHE in 2007 and 2013. The analysis shows an increase in the concentration index of $\mathrm{CHE}$ by $38.7 \%$, from -0.271 in 2007 to -0.376 in 2013. The negative concentration indices reveal that $\mathrm{CHE}$ is more concentrated among the less well-off (poor), meaning that the poor are more likely to incur CHE than the rich in Kenya.

Table 3.2: Concentration indices for Catastrophic Health Expenditure, 2007 and 2013

\begin{tabular}{llll}
\hline Year & Concentration Index $(\mathrm{Cl})$ & $\begin{array}{l}\text { Robust } \\
\text { Std. error }\end{array}$ & P Value \\
\hline 2007 & -0.271 & 0.036 & $<0.001^{* * *}$ \\
\hline 2013 & -0.376 & 0.028 & $<0.001^{* *}$ \\
\hline $\mathrm{Cl}_{2013}-\mathrm{Cl}_{2007}$ & -0.105 & & \\
\hline
\end{tabular}

The concentration curves in Figure 3.2 demonstrates the same, as they lie above the line of equality. This further shows that poor households spent a higher share of their household expenditure on healthcare services in 2007 and 2013 as compared to rich households.

Figure 3.2: Concentration curves for catastrophic health expenditure, 2007 and 2013

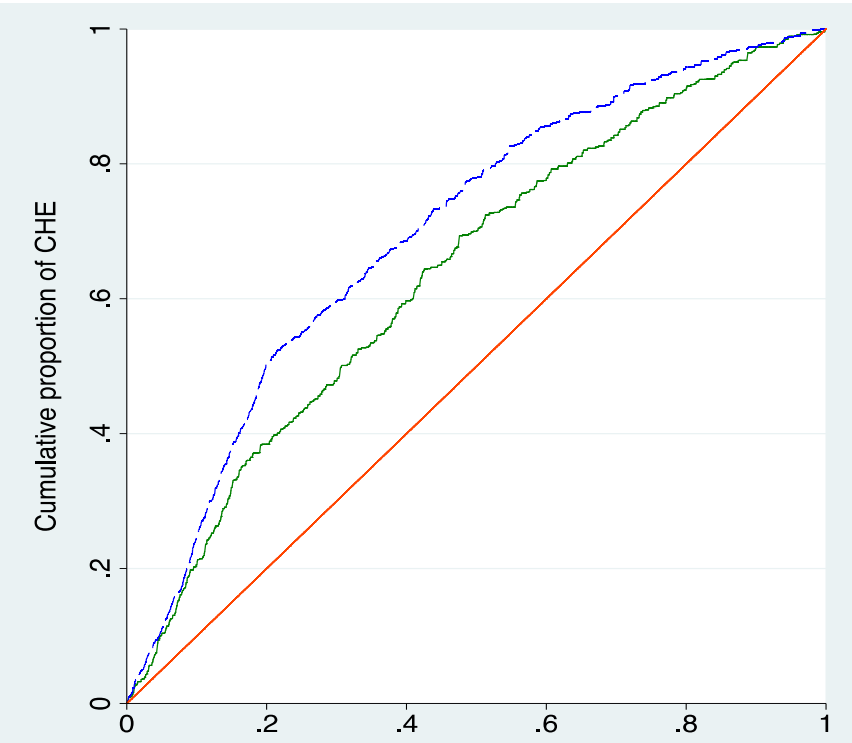

Cumulative prop. of households ranked [poorest to richest] 2007 2013 line of equality 


\subsubsection{Decomposition of socioeconomic inequalities in catastrophic health expenditure}

We decomposed the $\mathrm{CHE}$ concentration index against the socioeconomic variables to show the relative contribution of each variable to inequalities in $\mathrm{CHE}$, as shown in Table 3.3. The table presents the concentration index for each of the variables, the elasticity, the absolute contribution as well as the percentage contribution of each variable to the inequalities in $\mathrm{CHE}$.

The results show that, in 2007, the higher wealth quintiles, employed household heads, higher education status, and urban residence were positive contributors to inequality, meaning they favoured the well-off but disadvantaged the poor. Meanwhile, the insured households and private health providers, were the main negative contributors, implying they favoured the poor but disadvantaged the well-off.

In 2013, higher wealth quintiles and employed households' heads were the main positive contributors to inequality, implying they favoured the well-off, whereas insured household heads, and private health providers were negative contributors, meaning they favoured the worse-off.

Further, the results show that variables such as age of the household head, households with children under five, and seeking care from other providers in 2007 and age of the household head and medium-sized households in 2013 had minimal contribution to inequality, implying it was less sensitive to these variables. The unexplained factors were negative contributors of inequality, suggesting they tend to favour the worse-off, with their contribution to inequality decreasing from $11.4 \%$ in 2007 to $5.9 \%$ in 2013 . 
Table 3.3: Decomposition of the Inequalities in Catastrophic Health Expenditure, 2007 and 2013

\begin{tabular}{|c|c|c|c|c|c|c|c|c|}
\hline \multirow[b]{2}{*}{ Variables } & \multicolumn{4}{|l|}{2007} & \multicolumn{4}{|l|}{2013} \\
\hline & Elasticity & $\begin{array}{l}\text { Con. } \\
\text { Index }\end{array}$ & $\begin{array}{l}\text { Absolute } \\
\text { Contr. }\end{array}$ & $\begin{array}{l}\% \\
\text { Contr. }\end{array}$ & Elasticity & $\begin{array}{l}\text { Con. } \\
\text { Index }\end{array}$ & $\begin{array}{l}\text { Absolute } \\
\text { Contr. }\end{array}$ & $\begin{array}{l}\% \\
\text { Contr. }\end{array}$ \\
\hline \multicolumn{9}{|l|}{ Wealth status (Ref.=Poorest) } \\
\hline Second quintile & -0.185 & -0.398 & 0.074 & $-27.2 \%$ & -0.321 & -0.399 & 0.128 & $-30.5 \%$ \\
\hline Middle quintile & -0.214 & 0.030 & -0.006 & $2.4 \%$ & -0.357 & 0.000 & 0.000 & $0.0 \%$ \\
\hline Fourth quintile & -0.263 & 0.434 & -0.114 & $42.1 \%$ & -0.482 & 0.400 & -0.192 & $51.2 \%$ \\
\hline Richest quintile & -0.284 & 0.817 & -0.232 & $85.5 \%$ & -0.576 & 0.800 & -0.461 & $122.4 \%$ \\
\hline Female headed household & -0.064 & -0.156 & 0.010 & $-3.7 \%$ & -0.051 & -0.072 & 0.004 & $0.9 \%$ \\
\hline \multicolumn{9}{|c|}{ Age group of household head(Ref.=Below 25 years) } \\
\hline $25-40$ years & 0.020 & 0.060 & 0.001 & $-0.5 \%$ & 0.005 & 0.006 & 0.000 & $-1.3 \%$ \\
\hline $40+$ years & 0.005 & -0.038 & 0.000 & $0.1 \%$ & 0.042 & -0.007 & 0.000 & $1.0 \%$ \\
\hline \multicolumn{9}{|c|}{ Education of household head (Ref.=No education) } \\
\hline Primary education & -0.133 & -0.093 & 0.012 & $-4.6 \%$ & -0.028 & -0.166 & 0.004 & $-1.1 \%$ \\
\hline Secondary education & -0.089 & 0.243 & -0.021 & $7.9 \%$ & -0.021 & 0.175 & -0.003 & $0.0 \%$ \\
\hline Tertiary education & -0.016 & 0.625 & -0.009 & $3.6 \%$ & -0.007 & 0.548 & -0.004 & $0.1 \%$ \\
\hline Employed Household head & -0.335 & 0.046 & -0.015 & $5.6 \%$ & -0.931 & 0.026 & -0.024 & $6.4 \%$ \\
\hline Household wt. U5 Children & -0.012 & -0.002 & 0.000 & $0.0 \%$ & -0.064 & -0.069 & -0.004 & $-1.2 \%$ \\
\hline Household wt. Elderly (60+) & 0.024 & -0.156 & -0.004 & $1.4 \%$ & -0.1046 & -0.103 & 0.005 & $1.3 \%$ \\
\hline \multicolumn{9}{|c|}{ Household size (Ref.=1-3 small HH) } \\
\hline 4-6 Medium size & -0.093 & 0.035 & -0.003 & $1.2 \%$ & -0.065 & 0.001 & -0.001 & $0.3 \%$ \\
\hline 7+ Large size & 0.016 & 0.039 & 0.001 & $-0.2 \%$ & -0.135 & -0.084 & 0.011 & $-3.0 \%$ \\
\hline Urban residence & -0.037 & 0.396 & -0.015 & $5.5 \%$ & 0.072 & 0.262 & 0.019 & $-5.1 \%$ \\
\hline Insured households & 0.017 & 0.105 & 0.002 & $-0.7 \%$ & 0.214 & 0.392 & 0.084 & $-22.3 \%$ \\
\hline \multicolumn{9}{|c|}{ Type of health provider (Ref.=Public providers) } \\
\hline Private providers & 0.141 & 0.106 & 0.015 & $-5.5 \%$ & 0.257 & 0.153 & 0.039 & $-10.5 \%$ \\
\hline Other providers & -0.028 & 0.008 & 0.0000 & $0.1 \%$ & 0.010 & -0.039 & 0.000 & $0.1 \%$ \\
\hline $\begin{array}{l}\text { Household wt. member wt. } \\
\text { Chronic illness }\end{array}$ & 0.101 & 0.017 & 0.002 & $-0.7 \%$ & 0.194 & -0.016 & -0.003 & $-0.9 \%$ \\
\hline \multicolumn{9}{|l|}{ Constant } \\
\hline Residual & & & 0.031 & $-11.4 \%$ & & & 0.022 & $-5.9 \%$ \\
\hline
\end{tabular}




\subsubsection{Oaxaca-type decomposition of the change in inequalities for catastrophic health expenditure}

The decomposition of the total change in inequalities between 2007 and 2013 is presented in Table 3.4.

Table 3.4: Oaxaca-type Decomposition of the Change in Inequality for Catastrophic Health Expenditure, 2007 and 2013

\begin{tabular}{|c|c|c|c|c|c|c|}
\hline \multirow{2}{*}{ Variables } & \multicolumn{2}{|c|}{ Variation 1(Eq. 7) } & \multicolumn{2}{|c|}{ Variation 2(Eq. 8) } & \multicolumn{2}{|l|}{ Total } \\
\hline & $\Delta \mathrm{C} . \eta_{\mathrm{kt}}$ & $\Delta \eta \cdot C_{k t-1}$ & $\Delta \mathrm{C} . \eta_{\mathrm{kt}-1}$ & $\Delta \eta \cdot C_{k t}$ & Total & $\%$ \\
\hline \multicolumn{7}{|l|}{ Wealth status } \\
\hline Second quintile & 0.001 & 0.054 & 0.000 & 0.054 & 0.055 & $-52.4 \%$ \\
\hline Middle quintile & 0.011 & -0.004 & 0.006 & 0.000 & 0.006 & $-5.7 \%$ \\
\hline Fourth quintile & 0.016 & -0.095 & 0.009 & -0.088 & -0.079 & $75.2 \%$ \\
\hline Richest quintile & 0.010 & -0.239 & 0.005 & -0.234 & -0.229 & $218.1 \%$ \\
\hline Female head household & -0.004 & -0.002 & -0.005 & -0.001 & -0.006 & $5.7 \%$ \\
\hline \multicolumn{7}{|l|}{ Education of household head } \\
\hline Primary education & 0.002 & -0.009 & 0.01 & -0.017 & -0.007 & $6.7 \%$ \\
\hline Secondary education & 0.002 & 0.017 & 0.007 & 0.012 & 0.018 & $-17.1 \%$ \\
\hline Tertiary education & -0.001 & 0.014 & 0.001 & 0.012 & 0.014 & $-13.3 \%$ \\
\hline \multicolumn{7}{|l|}{ Age group of household head } \\
\hline $25-40$ years & 0.000 & -0.002 & -0.002 & 0.000 & -0.001 & $1.0 \%$ \\
\hline $40+$ years & 0.002 & -0.001 & 0.000 & 0.000 & 0.001 & $-1.0 \%$ \\
\hline Employed Household head & 0.022 & -0.029 & 0.008 & -0.015 & -0.007 & $6.7 \%$ \\
\hline Household wt. U5 Children & 0.004 & 0.000 & 0.001 & 0.004 & 0.005 & $-4.8 \%$ \\
\hline Household wt. Elderly $(60+)$ & 0.003 & -0.004 & 0.002 & -0.002 & -0.001 & $1.0 \%$ \\
\hline \multicolumn{7}{|l|}{ Household size } \\
\hline 4-6 Medium $\mathrm{HH}$ & 0.001 & 0.001 & 0.002 & 0.000 & 0.002 & $-1.9 \%$ \\
\hline 7+ Large $\mathrm{HH}$ & 0.017 & -0.006 & -0.002 & 0.013 & 0.011 & $-10.5 \%$ \\
\hline Urban residence & -0.010 & 0.044 & 0.005 & 0.030 & 0.034 & $-32.4 \%$ \\
\hline Insured households & 0.063 & 0.020 & 0.005 & 0.078 & 0.083 & $-79.0 \%$ \\
\hline \multicolumn{7}{|l|}{ Type of health provider } \\
\hline Private providers & 0.012 & 0.013 & 0.006 & 0.018 & 0.025 & $-23.8 \%$ \\
\hline Other providers & 0.000 & 0.000 & 0.001 & -0.002 & 0.000 & $0.0 \%$ \\
\hline $\begin{array}{l}\text { Household wt. member wt. } \\
\text { Chronic illness }\end{array}$ & 0.003 & 0.001 & 0.001 & 0.002 & 0.003 & $-2.9 \%$ \\
\hline Totals & 0.151 & -0.228 & 0.059 & -0.138 & -0.076 & $72.4 \%$ \\
\hline Residual & & & & & -0.029 & $27.6 \%$ \\
\hline Difference $\left(\mathrm{Cl}_{\mathrm{t}}-\mathrm{Cl}_{\mathrm{t}-1}\right)$ & & & & & -0.105 & $100.0 \%$ \\
\hline
\end{tabular}

E: Elasticity, Cl: Concentration Index, t:2013, (t-1):2007; Eq: Equation 
Overall, there was $-0.105(39 \%)$ increase in socioeconomic inequality in CHE between 2007 and 2013. The observed socioeconomic characteristics accounted for $72.4 \%$ of the change in inequalities in $\mathrm{CHE}$, while the remaining $27.6 \%$ was due to unobserved characteristics (residual).

High wealth quintile was the main contributor to the change in inequalities between 2007 and 2013, contributing to $218.1 \%$ increase. We observe that it is the change in elasticities of the wealth status that accounted for the significant increase in $\mathrm{CHE}$ inequalities, the bulk being attributable to the high wealth quintile. Insurance status of the household was the second variable that accounted for the most significant change in $\mathrm{CHE}$ inequalities, contributing $79 \%$ to lessening the inequalities. Other variables that contributed to lessening the inequalities included urban residence $(32.4 \%)$, private health providers $(23.8 \%)$, education level of the household head $(17.1 \%$ and $13.3 \%)$, and larger household size (10.5\%).

\subsection{Discussion}

The results show a decline in the incidence of CHE between 2007 and 2013, which is consistent with the national report in Kenya (MOH, 2014a). Determinants that lowered the odds of incurring CHE included higher wealth status, employed household heads and larger households. This is corroborated in Chapter 2 of this dissertation, that in the Sub-Saharan region, people with upper economic status, households with employed heads, and larger households are less likely to experience financial burden (Dyer et al., 2013). Seeking care from a private provider and having a household member with chronic illness increased the odds of incurring CHE. It can be inferred that this is because private healthcare providers charge high fees (Boing et al., 2014) and chronic illnesses are associated with long-term treatment costs (Q. Wang et al., 2016). Contrary to expectation, households with insurance increased the odds of incurring CHE in 2013. This is likely because the NHIF did not cover outpatient services by 2013 when both studies were conducted (E. Barasa et al., 2017). Additionally, in 2013, the insured had a higher utilisation rate for inpatient services, compared to the uninsured ( $\mathrm{MOH}, 2014 a$ ). Furthermore, evidence suggests that health insurance in itself can increase the risk of high catastrophic spending by encouraging individuals to seek care from high-level providers (Ghafoori et al., 2014; Lindelow \& Wagstaff, 2005).

Despite the decrease in the incidence of $\mathrm{CHE}$, inequalities in $\mathrm{CHE}$ increased over time. This implies that socioeconomic inequalities in catastrophic expenditure have worsened in Kenya. This is corroborated by other studies in Kenya that shows inequalities in CHE still exist and are disproportionately concentrated amongst the worseoff (E. Barasa et al., 2017; Salari et al., 2019). Furthermore, the results show that the better-off experienced a higher percentage of decline in CHE compared to the worse-off.

Higher wealth quintiles and employed household head were the main contributors of inequalities in CHE in both 2007 and 2013. Poorer households in Kenya are reported 
to spend more OOP in proportion to consumption than their rich counterparts (Chuma \& Maina, 2012). The unemployed have no or little income, which restricts their access to healthcare (Woolf et al., 2015), Insured households and private health providers contributed to pro-rich inequality in CHE; furthermore, high socioeconomic status is associated with higher odds of insurance coverage (Kazungu \& Barasa, 2017). Evidence suggests overuse of healthcare services amongst households that are insured and low utilisation amongst the non-insured (Acharya et al., 2013; Adam Wagstaff et al., 2018). $A$ recent study in Kenya revealed that pro-rich inequality in health service use is significantly higher for care provided in privately owned facilities (Ilinca et al., 2019).

Changes in inequalities and elasticities of the socioeconomic determinants largely explain the change in socioeconomic inequalities in CHE. The analysis suggests that there are unobserved factors that contributed to the change in inequalities. Household wealth status was the main contributor to the rise in inequalities in CHE. This points to the existence of significant inequalities in Kenya not only in health but across various sectors and geographical regions. For instance, more than half (59.4\%) of the country's total expenditure is controlled by the richest quintile while the poorest quintile controls only 3.6 per cent. Also, the largest share of household expenditures is controlled by the fourth and richest quintile (KNBS, 2018b). Changes in inequality in a household's insurance status accounted for a substantial decrease in the change in CHE inequalities. This could be because of the decline in health insurance coverage inequalities in Kenya (Kazungu \& Barasa, 2017). This underscores the critical role of health insurance coverage in reducing inequalities in the financial burden imposed by healthcare. Emphasising the marked rural-urban inequalities that exist in Kenya (Arndt et al., 2016), inequalities relating to rural-urban residence was important in explaining the change in $\mathrm{CHE}$ inequalities. The contribution of larger household size to reducing inequalities stressed the role of social capital through the pooling of resources among several households or community members, which is vital in the African communal setting and the large informal workforce (Buigut et al., 2015; N. K. David et al., 2017).

Education is critical in enhancing health outcomes by reducing the need for healthcare and associated costs of dependence (Feinstein et al., 2006). Our results show that the education level of the household head was attributed to reducing inequalities over time. This is undoubtedly due to the multiplier effect of education given educated household heads are likely to be employed and thus have better economic status and better access to healthcare services (Zimmerman \& Woolf, 2014). Furthermore, our descriptive analysis indicates an improvement in the education level of the household heads between the surveys, with up to $74 \%$ in 2013 , having at least primary level education.

Overall, the results underscore the existence of socioeconomic inequalities in Kenya that disadvantage the poor. In accordance with other studies, the results emphasise that the drivers of socioeconomic inequalities in healthcare payments extend to other social sectors beyond the health sector (Ilinca et al., 2019). Despite Kenya 
showing progress in addressing poverty, the burden on the poor is still significantly exacerbated by these relatively high and persistent inequalities (KNBS, 2018b). Collaboration across sectors such as social protection could offer more significant impact in financial risk protection for the most vulnerable hence reduce socioeconomicrelated inequalities which if left unchecked could reverse the gains in the health sector. Analysis of inequalities in healthcare access is therefore vital as it unravels existing nuances and variations across socioeconomic groups. Furthermore, it is possible to have national averages decrease but mask disparities amongst subgroups (WHO, 2013). For instance, as shown in this study, although the incidence of CHE declined over time, the inequalities affecting the poor have deteriorated.

There are limitations that need to be considered while interpreting the findings. First, in both surveys, data on outpatient health expenditure was collected based on the last 4 weeks preceding the survey, whereas the inpatient expenditure was annual. To have a standard period of reference, we annualised the outpatient expenditure as per practice. This could possibly overestimate or underestimate healthcare expenditures. Second, the expenditure in the surveys are all self-reported and may suffer from recall bias. Third, the timing of the study and seasonality may have implications on the type of illness and health-seeking behaviour, given that some illnesses are prevalent at certain times of the year, subsequently having implications on the cost burden at the household level (Sauerborn et al., 1996).

\subsection{Conclusions}

Socioeconomic inequalities in CHE persist in Kenya, suggesting that the existing financial risk protection mechanisms have not sufficiently addressed these disparities. The recent changes to the National Social Health Insurance Fund (NSHIF) is a positive move, but it is based on a premium; thus, many poor households may still lack the ability to pay. There are still opportunities to improve on pro-poor mechanisms through the UHC initiatives hence address socioeconomic inequalities in the utilisation of healthcare services in Kenya.

This study demonstrates that understanding the socioeconomic factors that sustain inequalities in CHE is paramount in informing policymakers of the need to intensify and tailor pro-poor interventions. A fundamental lesson from the findings is that the drivers of inequalities, such as economic status and unemployment, extend beyond the health sector. Therefore, a multi-sectoral approach should be considered in addressing socioeconomic inequalities so as to draw synergies and efficiencies across various sectors hence accelerate the achievement of UHC. 


\section{Chapter 4 :}

\section{A Multilevel Analysis of Cost-Related Unmet Need for Healthcare Services in Kenya}

Based upon:

Njagi Purity, Arsenijevic Jelena, Groot Wim 2020; Cost-related unmet need for healthcare services in Kenya. BMC Healthe Services Research, 20(1). https://doi.org/10.1186/s12913$\underline{\text { 020-05189-3 }}$ 


\section{Abstract:}

Background: The assessment of unmet need is one way to gauge inequities in access to healthcare services. While there are multiple reasons for unmet need, financial barriers are a major reason particularly in low- and middle-income countries where healthcare systems do not offer financial risk protection. Moreover, accessibility and affordability are paramount in achieving universal health coverage. This study examines the extent of unmet need in Kenya due to financial barriers, the associated determinants, and the influence of regional variations.

Methods: We use data from the 2013 Kenya household health expenditure and utilization (KHHEUS) cross sectional survey. Self-reported unmet need due to lack of money and high costs of care is used to compute the outcome of interest. A multilevel regression model is employed to assess the determinants of cost-related unmet need, confounding for the effect of variations at the regional level.

Results: Cost-related barriers are the main cause of unmet need for outpatient and inpatient services, with wide variations across the counties. A positive association between county poverty rates and cost-related unmet is noted. Results reveal a higher intraclass correlation coefficient (ICC) of 0.359 (35.9\%) for inpatient services relative to 0.091(9.1\%) for outpatient services. Overall, differences between counties accounted for $9.4 \%$ (ICC 0.094) of the total variance in cost-related unmet need.

Conclusion: The findings underscore the important role of cost in enabling access to healthcare services. The county level is seen to have a significant influence on costrelated unmet need. The variations noted in cost-related unmet need across the counties signify the existence of wide disparities within and between counties. Scaling up of health financing mechanisms would fundamentally require a multi-layered approach with a focus on the relatively poor counties to address the variations in access. Further segmentation of the population for better targeting of health financing policies is paramount, to address equity in access for the most vulnerable and marginalized populations. 


\subsection{Introduction}

As described in Chapter 2, individuals may refrain from seeking care due to catastrophic payments. Delaying or forgoing treatment may have negative effects on health status among different population groups (Chen et al., 2011). Furthermore, forgoing necessary treatment might impose a financial burden in the long run, or even lead to poorer health. At least half of the world's 7.3 billion population are reported to not receive the essential healthcare services they need (WHO \& World Bank, 2017). This is more profound in developing countries where many people go without healthcare from which they could benefit greatly (O'Donnell, 2007). The reasons for forgoing healthcare can be found in the numerous barriers that hinder access such as (Jacobs et al., 2012) financial, organizational, social, psychological or cultural barriers. These barriers may limit use of healthcare services and impose large healthcare coverage gaps among different population groups (Gulliford \& Morgan, 2003).

One method of gauging equity of access to services is through assessing unmet need for healthcare (Fiorillo, 2019; OECD, 2011). Unmet need is implicitly defined as the difference between the services judged necessary and the services actually received, resulting from barriers related to accessibility, availability and acceptability (Fjær et al., 2017). There are two recommended approaches for measuring unmet need. On the one hand, one can use a 'clinical' assessment based on relevant clinical guidelines. On the other hand, there is a 'subjective' measure based on the individual's own assessment not to have received healthcare when needed because of access barriers beyond their control (Allin, Grignon, et al., 2010). The subjective measure is a widely used approach due to its feasibility as many surveys include self-reports by individuals on the time, they needed care but did not receive it (Allin \& Masseria, 2009b).

Poor functioning health financing systems and the cost of healthcare services may deter people from seeking healthcare when they need it in order to avoid financial burden. This is especially observed when direct OOP payment are involved (Boerma, 2015; Swe et al., 2018). Financial access is thus critical, given that it can lead to catastrophic costs and/or impoverishment (Mchenga et al., 2017; Xu et al., 2003a). When there are no direct costs at the time of access to healthcare services ( (OOP) for example), then catastrophic health expenditure can be prevented (Ezat Wan Puteh \& Almualm, 2017). Many studies have used catastrophic health expenditure to gauge the extent of financial risk protection. However, given $\mathrm{CHE}$ occurs in the form of direct and indirect health expenditure it is only incurred if sick individuals actually seek the needed healthcare. This means that the small incidence of $\mathrm{CHE}$ in some countries may create the impression of a greater degree of financial risk protection than what it provided by the system (Mchenga et al., 2017). Furthermore, the analysis of CHE may suffer from selection bias. For instance a low incidence of catastrophic spending might simply reflect a situation in which only a few people get the healthcare they need (Adam Wagstaff et al., 2018), and we do not observe people who need care but are unable to get it. 
In case of the Kenya healthcare system, inequities in accessing the healthcare services can be described as problems in regional discrepancies in the health service distribution, disparities in resource allocations, and inequitable access to quality healthcare services (Ndavi et al., 2009; Nyanjom, 2006). Significant regional inequities remain where northern counties, rural households and ethnic minorities are reported to have worse healthcare coverage (ODI, 2016). Recent analysis show regional disparities in access, in that some regions are less likely to go without medical care, while other regions experience difficulty in obtaining medical care (Jivetti et al., 2018; Mugo et al., 2018).

The inequities in accessing healthcare services are also related to unmet need. A large variation in unmet healthcare needs is reported across counties ranging between $4.1 \%$ to $40.4 \%$, with the cost being one of the top reasons why people forgo care $(\mathrm{MOH}$, 2014a). Furthermore the counties vary by socioeconomic composition, as some are predominantly rural while others are urban (CRA, 2011). Additionally, the numbers of those who do not seek care in Kenya due to costs is particularly high in the rural areas (Chuma et al., 2007). Studies on unmet need for care in Kenya and the region have primarily examined overall reasons for unmet need for specific healthcare services, particularly reproductive services (Ayanore et al., 2016; Ettarh, 2011; Guure et al., 2019; Machiyama \& Cleland, 2013). Others have focused on barriers to utilization of services such as non-availability of drugs, staffing inadequacy among others (Muhammed et al., 2013; Tey \& Lai, 2013). To our knowledge, there has been no study that has looked at the factors influencing unmet need for healthcare due to costs alone. Analysis of the reasons for unmet need can be important in focusing policy actions (Allin, Grignon, et al., 2010). This study adds to the existing literature on financial access by first, examining the extent of cost-related unmet need for healthcare and second, explaining how much of the cost-related unmet need is attributable to the differences in individual characteristics, and the effect of the variations at the regional (county) level.

Geographical assessment of unmet need is critical in the Kenyan context given the recent (2013) decentralisation of the healthcare system to new sub-national governance units (counties). Moreover, the counties have the responsibility for healthcare financing and provision of services. This Chapter applies a multi-level regression analysis model to provide for factors that are contextual at the individual/household level, adjusting for variations at the county level. Furthermore, many empirical studies are reported to focus on individual level and/or aggregate level inequities and not paying attention to multilevel structures (Subramanian et al., 2001). A multi-level analysis provides a more nuanced understanding of the drivers that are influential at the different levels. Disaggregating unmet need discerns those reasons that are relevant to policymakers, and those reflecting individuals' and households' preferences and taste (Allin \& Masseria, 2009b). The findings of this study are relevant in informing the healthcare financial reforms in Kenya both at the national and at the county level, to better improve access to services to underserved populations, and hence 
accelerate achievement of UHC across all regions.

\subsection{Methods}

\subsubsection{Data source and sample}

This study uses data from the KHHES implemented by the Ministry of Health in collaboration with Kenya National bureau of statistic every five years. This is a national representative household survey aimed at collecting data on household healthcare utilization. The survey collects data on individuals who were ill, those who sought care, and the reasons for not seeking care when ill. Other data collected include demographic and socioeconomic characteristics of the individuals and households. This study utilizes data from the 2013 survey which covered Kenya's 44 out of the 47 counties. A total of 33,675 households (152,566 individuals) were interviewed from 1,347 selected clusters; 814 (60 percent) were rural and 533 (40 percent) urban clusters with a response rate of $87.7 \%$.

\section{Outcome variable}

Unmet need for healthcare is seen to cover a spectrum of healthcare needs that are not optimally met. On the one hand is "unexpressed demand" referring to people with healthcare needs who are not aware of them, or who do not to seek healthcare. On the other hand is "expressed demand" referring to healthcare needs that are suboptimally met (Academy of Medical Sciences, 2017).

This study uses a dependent (outcome) variable related to "unexpressed demand", which is the subjective perception of not receiving appropriate care when needed (Fjær et al., 2017). We look at whether people with healthcare needs choose not to seek care due to the high cost of care and lack of money, referred to as self-reported "cost-related unmet need' for healthcare services. We construct the cost-related unmet need for healthcare variable from two sets of questions:

(i) Outpatient - Was <NAME> ill in the last four weeks? If Yes, ..... did <name> visit/consult a health provider?

(ii) Inpatient - Did [Name] need to be admitted in a hospital in the last twelve months? If Yes ......, was <name> admitted?

The reasons why healthcare was not sought when needed are derived from two closed ended questions framed as follows; Outpatient - "If No ......, what was <name>'s main reasons for not seeking care?" and Inpatient "If No ......., why was <name> not admitted?". The responses are a pre-defined set of multiple-choice categories allowing for multiple responses. They include lacked money, prescribed drugs were not available, self-medication, poor quality of service, high cost of care, religious/cultural issues, fear of discovering serious illness, long distance to the provider and illness not considered serious enough. 


\section{Explanatory variables}

Based on Andersen's Health Behaviour Model we distinguish three groups of factors that affect access to healthcare services: need, predisposing and enabling factors (R M Andersen et al., 1983; Babitsch et al., 2012). We thus classify the independent variables into (i) predisposing factors such as: age of household head, gender of household head, education level of household head and employment status of household head, (ii) need factors such as: type of service sought (inpatient/outpatient care), self-rated health status and chronic illness, and (iii) enabling factors such as: household wealth, household size, insurance status, and residence (rural/urban).

\subsubsection{Multilevel regression model}

This study applies a multilevel logistic regression model to analyse the association and variability between cost-related unmet need for care, and the independent factors. Multilevel analysis is preferred given that it allows for the simultaneous examination of the effects of group-level and individual-level factors and groups are perceived as related, that is coming from a larger population of groups (Diez-Roux, 2000). Additionally, multilevel analysis accounts for dependencies of observations within groups (Roux, 2008). Multilevel (logistic) regression disentangles the within-cluster effects from the between-cluster effects (Sommet \& Morselli, 2017). Relative to conventional models, multilevel models provide a more accurate and comprehensive description of relationships in clustered data (Park \& Lake, 2005). In this study, individuals and households are nested within regions known as counties, thus we use counties as the group level variable. First, we present the proportion of people who face unmet need and the different reasons for unmet needs (see Table 4.1), then we present descriptive statistics for unmet need related to costs (see Figure 4.1 and Figure 4.2), and the results related to logistic multilevel regression (see Table 4.3). We fit a Pearson's correlation coefficient test statistic to assess the association between poverty rates, and costrelated unmet need for healthcare services. A log likelihood ratio test is used to assess the goodness of fit between the general logistic model and the multilevel model. Additionally, this study applies two commonly used information criteria - Alkaike's information Criterion(AIC) and the Bayesian Information Criterion (BIC), to assess the relative model goodness of fit (Burnham \& Anderson, 2004).

\subsection{Results}

\subsubsection{Proportion of population with unmet need for healthcare services}

The analyses show that out of those who needed healthcare services $88.3 \%$ needed outpatient services within the last 4 weeks, whereas $11.7 \%$ needed inpatient services within the last 12 months. However, 8.3\% did not seek the needed healthcare services. Unmet need for healthcare services was higher among those that needed inpatient services at $9.3 \%$, relative to those that needed outpatient services at $8.2 \%$. Table 4.1 shows the proportion with unmet need, and the various reasons for unmet 
need for healthcare services. The top reasons why people had to forgo care were costrelated (38.6\%), IIIness not considered serious (33.5\%), and self-medication (19.6\%). Cost-related reason was higher for inpatient services (56.5\%) compared to outpatient services (35.5\%).

Table 4.1: Unmet need for healthcare services and the reasons for unmet need

\begin{tabular}{|c|c|c|c|c|c|c|}
\hline \multirow[b]{2}{*}{ Variables } & \multicolumn{2}{|c|}{ Outpatient } & \multicolumn{2}{|c|}{ Inpatient } & \multicolumn{2}{|l|}{ Total } \\
\hline & $\mathrm{N}$ & $\%$ & $\mathrm{~N}$ & $\%$ & $\mathrm{~N}$ & $\%$ \\
\hline Was ill and Needed admission & 36,901 & 88.3 & 4,882 & 11.7 & 41,783 & \\
\hline Did not seek healthcare services & 3,009 & 86.9 & 453 & 13.1 & 3,462 & \\
\hline Percentage with unmet need & & 8.2 & & 9.3 & & 8.3 \\
\hline Percentage with cost-related unmet need & & 2.9 & & 5.2 & & 3.2 \\
\hline \multicolumn{7}{|l|}{ Reasons for unmet need } \\
\hline Cost-related (Lack of money and high costs) & 1,081 & 35.9 & 256 & 56.5 & 1,337 & 38.6 \\
\hline Illness was not considered serious & 1,070 & 35.6 & 88 & 19.4 & 1,158 & 33.5 \\
\hline Self-Medication & 646 & 21.5 & 32 & 7.1 & 678 & 19.6 \\
\hline Long distance to the health provider & 49 & 1.6 & 8 & 1.8 & 57 & 1.7 \\
\hline Religious/cultural reasons & 36 & 1.2 & 5 & 1.1 & 41 & 1.2 \\
\hline Fear of serious illness & 11 & 0.4 & 1 & 0.2 & 12 & 0.4 \\
\hline Perceived poor quality of service & 8 & 0.3 & 4 & 0.9 & 12 & 0.4 \\
\hline Perceived lack of drugs at the facilities & 10 & 0.3 & 4 & 0.9 & 14 & 0.4 \\
\hline Other reasons (Not specified) & 98 & 3.3 & 55 & 12.1 & 153 & 4.4 \\
\hline Total (N) & 3,009 & & 453 & & 3,462 & \\
\hline
\end{tabular}

Of those who needed outpatient or inpatient services, $3.2 \%$ did not seek the needed healthcare services due to cost, that is high cost of the services and/or lacked the money. Our analysis show that cost-related unmet need is higher for inpatient services at 5.2\% compared to the outpatient services at 2.9\%. Appendix 4-A further presents the distribution across counties of those that did not seek healthcare services due to cost-related reasons, and other reasons including no drugs, self-medication, poor quality service, religious/cultural reasons, fear that the illness is serious, distance to provider and illness not considered serious. 


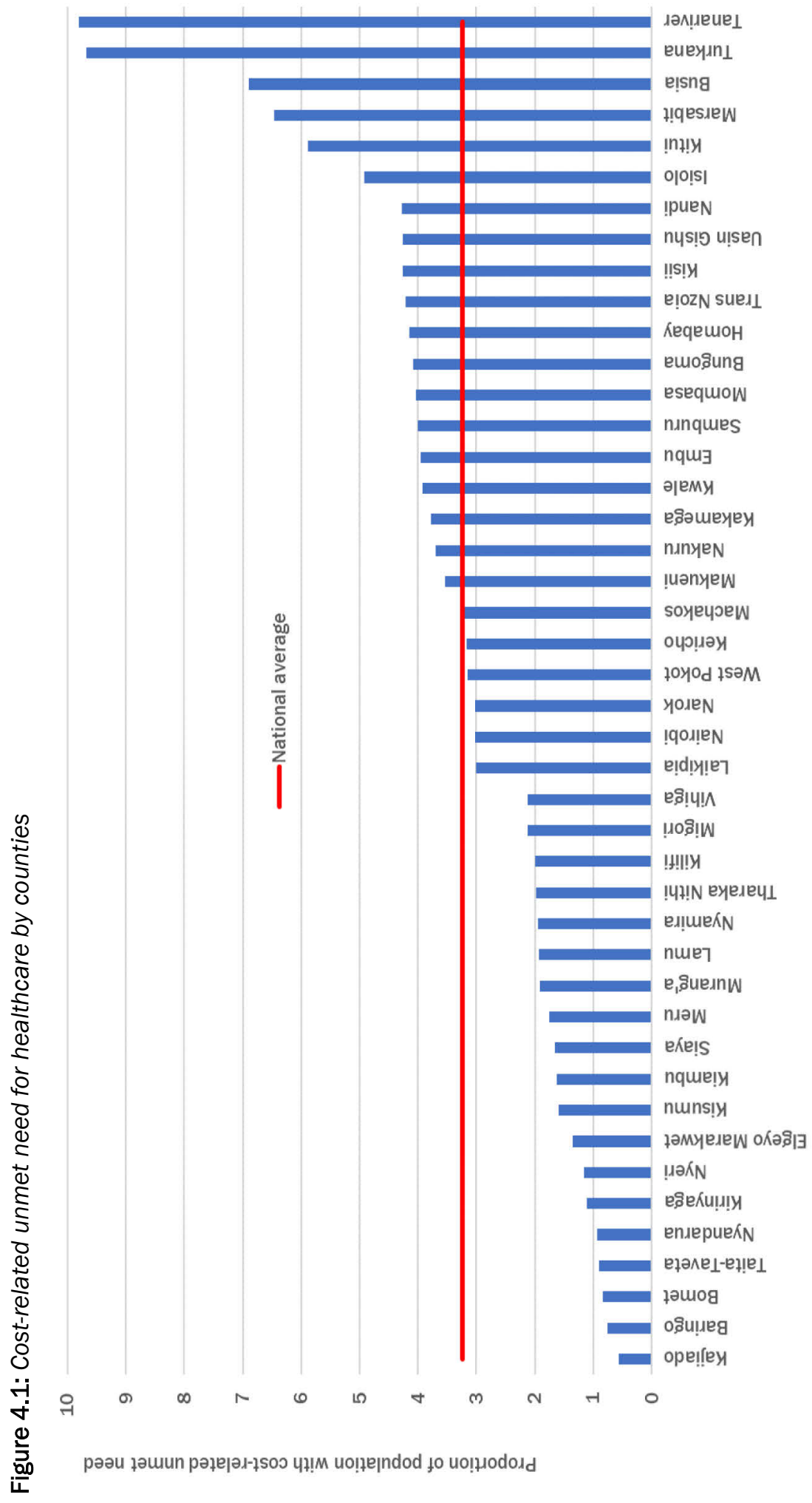


Figure 4.1 shows the distribution of those who experienced cost-related unmet need by counties. The analysis shows a wide variation in cost-related unmet need among the counties from $<1 \%$ to $9.8 \%$. Nearly half $(43 \%)$ of the counties had cost-related unmet need higher than the national average of $3.2 \%$. We observed that several counties with a prevalence of unmet need of $<1 \%$, had small samples $(\mathrm{N})$ of up to $<10$ persons with cost-related unmet need (See Appendix 4-A). In light of this, we run a sensitivity analysis on the multilevel model to assess the robustness of the findings without the 9 counties that have a cost-related unmet need of less than 10

\subsubsection{Descriptive analysis of the population with cost-related unmet need for healthcare}

The distribution of those who experienced cost-related unmet need for healthcare by the socio-economic characteristics is presented in Appendix 4-B. Households head above 40 years accounted for the majority of those who had cost-related unmet need (64.3\%). The majority (81.1\%) of those who experienced cost-related unmet need came from households with a head with primary education only. Persons with cost-related unmet need from male headed households were $68.1 \%$, similarly $82.5 \%$ with costrelated unmet need had an employed (formally and informally) household head. More than half $(56.3 \%)$ of those with cost-related unmet need self-rated their health as good. Only $26.2 \%$ of those with cost-related unmet need had chronic illness. Cost related unmet need for care was experienced by $42.3 \%$ and $31.7 \%$ of the people from medium and large size $(7+)$ households respectively. Only $7.3 \%$ of those who experience cost related unmet need for care were from insured households. Rural households accounted for majority $(70.3 \%)$ of the individuals who experienced cost-related unmet need for healthcare. Of the individuals who experienced cost-related unmet need for care, the majority were from poorest households (38.1\%), while the least were from among the richest households (4.8\%).

There was a statistically significant positive correlation (Pearson's r 0.638; p-value $<0.0001$ ) between the country poverty rates, and cost-related unmet need for healthcare. The majority of the counties that experienced high cost-related unmet need for healthcare had high poverty rates as shown in Figure 4.2. 


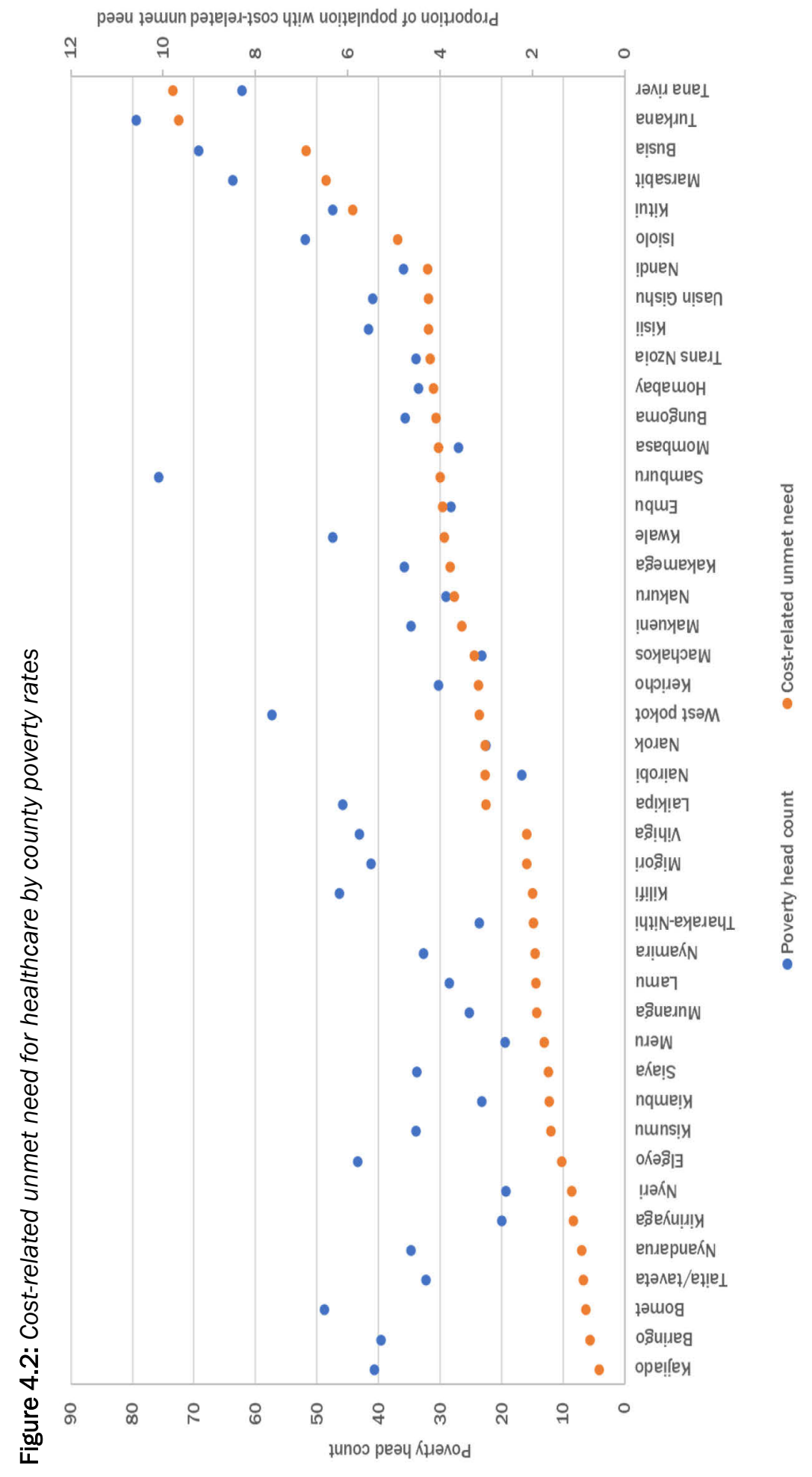

ㄱ 


\subsubsection{Multilevel analysis of cost-related unmet need for healthcare}

We fit both the general logistic regression model, and the multilevel regression model (see Appendix 4-C). Although the estimates from both models show small differences, the log likelihood test confirms that there is a statistically significant difference between the two models $\left(\mathrm{chi}^{2}=257.14\right.$, P-value $<0.001$ ). Relative to the logistic regression model, the multilevel model has the lowest AIC and BIC $(10,894.69$ and 11,076.07 respectively) which shows the performance of the model based on its log-likelihood indicating it is the better fitting model as illustrated in Table 4.2. This demonstrates that controlling for the county level variation leads to a significant improvement of the model, relative to running a general regression model. We therefore discuss the results from the multilevel model which controls for between counties' variation.

Table 4.2: AIC and BIC for the logistic and multilevel model

\begin{tabular}{|c|c|c|c|c|c|c|}
\hline & Obs & II(null) & II(model) & df & AlC & $\mathrm{BIC}$ \\
\hline Logistic Model & 41,646 & $\begin{array}{l}- \\
5,889.088\end{array}$ & $-5,554.914$ & 20 & $11,149.83$ & $11,322.57$ \\
\hline Multilevel Model & 41,646 & - & $-5,426.346$ & 21 & $10,894.69$ & $11,076.07$ \\
\hline
\end{tabular}

Table 4.3 presents the analysis from the multilevel logistic regression models. We fit three models including an outpatient model 1 , an inpatient model 2 , and an overall model 3 controlling for outpatient and inpatient services. The results show the association of the predisposing, need and enabling factors on cost-related unmet need for healthcare services at the individual level confounding for the county level. Overall, the results show an estimated intraclass correlation coefficient (ICC) of 0.094 , meaning that $9.4 \%$ of the variance in cost-related unmet need is attributable to county level variations. This is close to the 0.091 ICC reported in the outpatient model, indicating the county level accounted for $9.1 \%$ of the variation in outpatient cost-related unmet need. Equally, we note a higher ICC of 0.359 in the inpatient model, suggesting that county level variation accounts for $35.9 \%$ of the inpatient cost-related unmet need. Given that 9 counties had a very low sample $(<10)$ with cost-related unmet need, a separate multilevel model is fitted without these counties to assess the robustness of the findings. The intraclass correlation coefficient(ICC) is $6 \%$ relative to $9.4 \%$ in the multilevel model with all the counties. Additionally, the direction of the effect of the explanatory factors on cost-related unmet need is the same in both models. Furthermore, the confidence intervals for all the factors are overlapping meaning there is no significant difference between the two models. Finally, we run a log-likelihood ratio test which indicates no statistically significant difference between the two models.

The analysis shows a positive association between cost-related unmet need for healthcare and older household heads ( +40 years) in both the outpatient and overall model. Relative to households with younger heads (below 25 years), older households 
have $79 \%$ and $92 \%$ higher odds of unmet need for healthcare due to cost in the overall and outpatient model respectively. In addition, household heads with tertiary education have $47 \%$ and $52 \%$ lower odds of cost-related unmet need for healthcare relative to those with no education in the overall and outpatient model respectively. Although in the inpatient model being an employed households' head was associated with 33\% lower odds of experiencing cost-related unmet need, in the outpatient and overall model, employment status of the household head had no significant association with costrelated unmet need.

The overall model shows seeking inpatient services was associated with 95\% increase in the odds of cost-related unmet need. There was a negative association between good self-rated health and cost-related unmet need across the three models, with $44 \%$ reduced odds of unmet need for care due to costs in the overall model relative to poor self-rated health. Insured households were $50 \%$ less likely to have cost-related unmet relative to uninsured households, meaning the odds were even in the overall model. Household size was negatively associated with cost-related unmet need for care across the three models. For instance, medium and larger households had 31\% and 29\% lower odds of cost-related unmet need respectively, relative to those from small households.

Urban residents were $25 \%$ in the overall model and $28 \%$ in the outpatient model, more likely to have cost-related unmet need for care relative to their rural counterparts. However, there was no association between cost-related unmet need and residence in the inpatient model. In all the three models, wealth status of the household was negatively associated with the cost-related unmet need for healthcare with the likelihood reducing as you move up the wealth quintiles. In the overall model, households in the richest quintile were $69 \%$ less likely to have unmet needs, while for the, fourth quintile this was $53 \%$, the middle quintile had a $37 \%$, and second quintile had $15 \%$ less chance to have cost-related unmet need for healthcare relative to the those in the poorest quintile. 


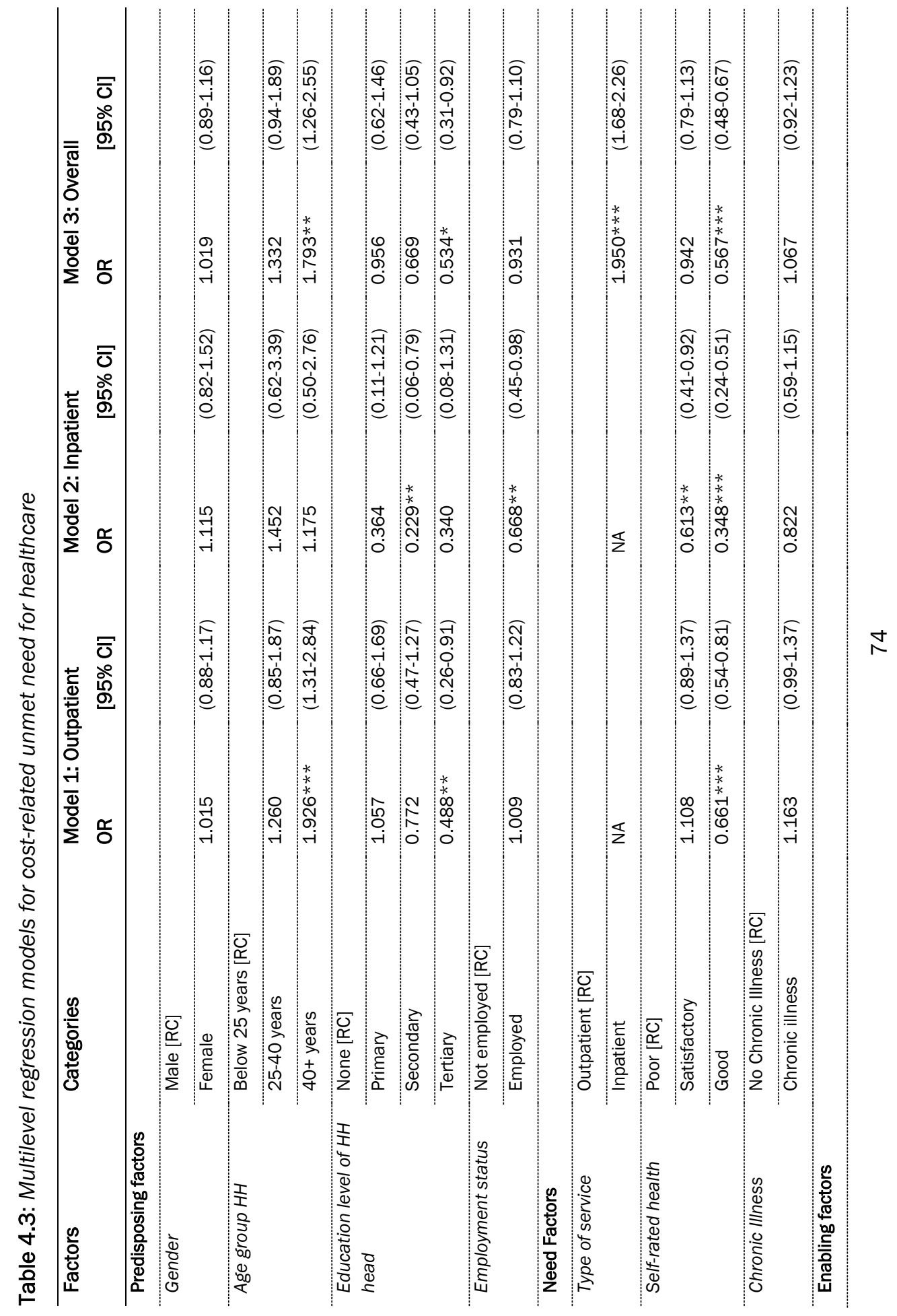




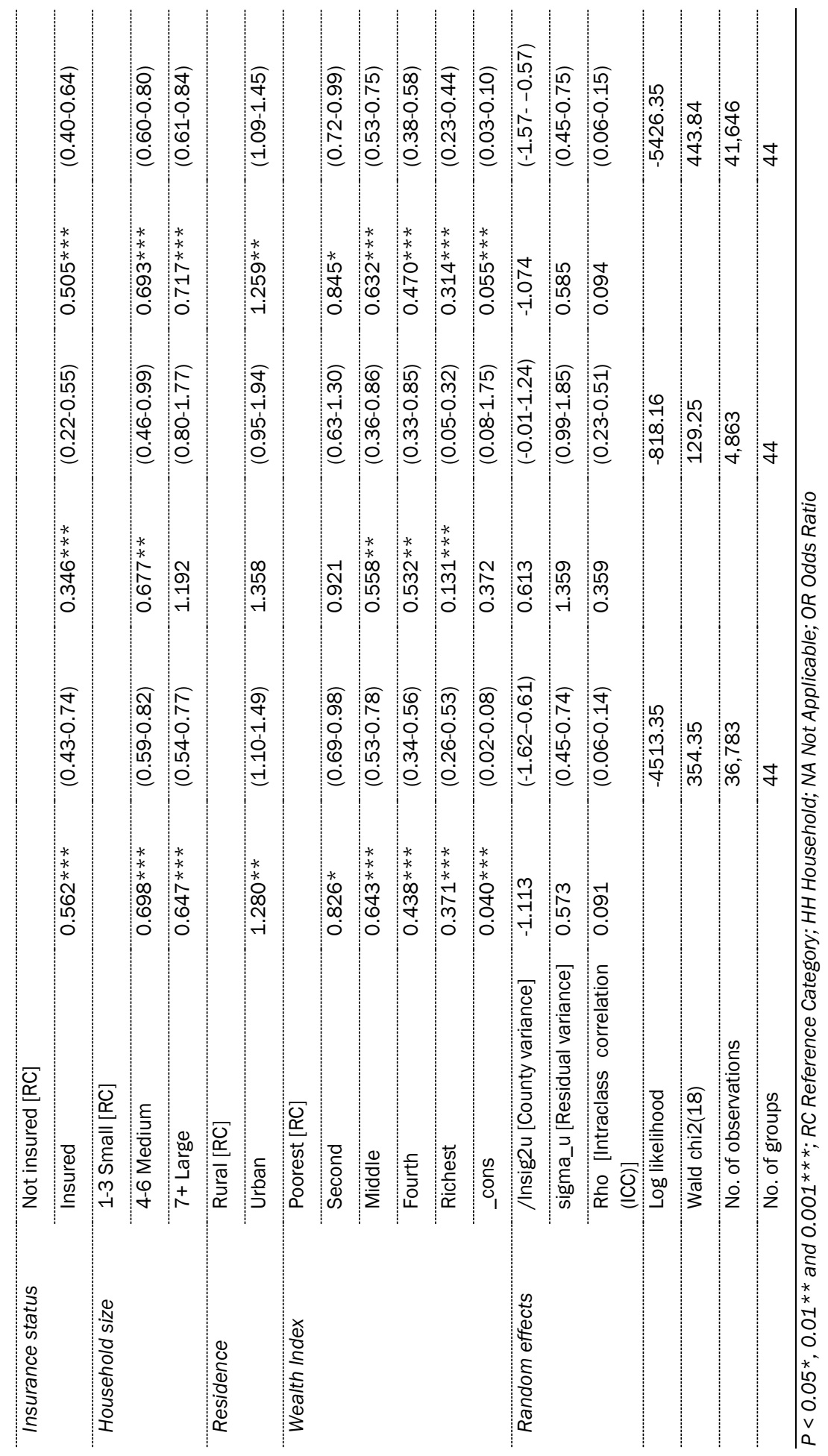




\subsection{Discussion}

The findings show that $8.3 \%$ of those who needed outpatient care or inpatient care did not seek healthcare services with inpatient unmet need being higher relative to outpatient unmet need. This is lower than the percentage for unmet need $-12.7 \%$ reported in the national report $(\mathrm{MOH}, 2014 \mathrm{a})$, given this study computed unmet need based on persons who provided a reason for not seeking outpatient or inpatient care. This means there are persons who did not provide any reason why they did not seek care when needed. Additionally, the national report highlights high cost among the top three most important reasons for not seeking care at $21.4 \%(\mathrm{MOH}, 2014 \mathrm{a})$. Our results emphasize cost as the major reason for not seeking healthcare at $38.6 \%$. This is in accordance with other studies in Kenya, and elsewhere that highlight cost as an important and most frequent reason for unmet need for care (Cavalieri, 2013; Chuma et al., 2007; Ngugi et al., 2017; Popovic et al., 2017).

The findings slightly differ from those of the national report because we define costrelated unmet need as a combination of two reasons "lacked money" and "high costs of care", whereas the national report focused on only the high cost of care. The lack of money could infer to other indirect costs of the service such as, transport costs like in rural areas where travel distances to the health facilities are longer (Ganesh, 2015; O'Donnell, 2007).

Overall, of those who needed outpatient or inpatient services, 3.2\% had to forgo the healthcare services due to cost-related issues (lack of money and high costs). We observe a higher cost-related unmet need for inpatient services relative to outpatient services. This could be because inpatient care is reported to be unaffordable to poor households because it is more costly than outpatient services (Chuma \& Maina, 2012).

Cost-related unmet need varies across the regions/counties ranging from $0.56 \%$ to $9.8 \%$. However, $43 \%$ of the 44 counties $(n=19)$ do report cost-related unmet need higher than the average in the whole country. This is consistent with other studies in Kenya that have highlighted that health outcomes remain heterogeneous at the county level, although with convergence across counties over time (Achoki et al., 2019). This means that cost-related unmet need might impose inequities in access to healthcare between counties. This could be also due to variation in socio-economic status across the counties. Our analysis also shows a positive association between the poverty rates of the counties, and cost-related unmet need for healthcare services. Consistent with other studies, results indicate that counties with high poverty rates had high cost-related unmet need, signifying that health costs are much more a burden to the poor (CastroLeal et al., 2000).

We assessed the predisposing, need and enabling factors that are associated with cost-related unmet need for care through both a general logistic regression, and multilevel model to account for country level variations. Although there is minimal difference in the estimates of the determinants between the two models, the test for goodness of fit indicate that the multilevel model is superior (better fit) relative to the 
logistic regression model.

The results indicate that differences between counties accounted for $35.9 \%$ of the total variance in inpatient services unmet need, compared to $9.1 \%$ in outpatient services unmet need. Furthermore, a wide variation is reported in per capita spending on inpatient care by county $(\mathrm{MOH}, 2014 \mathrm{a})$. This corroborates previous evidence that there exist inequities within the counties, and that Kenyans living in the same region could have different lifestyles and access to services (KNBS \& SID, 2013). Conversely, other studies have highlighted wider socio-economic and geographic inequities for inpatient care than outpatient care in Kenya (Chuma \& Okungu, 2011). However, there are likely to be more similarities among people within the same county in relation to costrelated unmet need for inpatient services relative to outpatient services. Contextual factors at the county level are likely to influence the individual factors on cost-related unmet need. For instance the degree of urbanity at county level, bearing in mind some counties are more urbanised than others and the rural urban disparities in Kenya (Friedrich-Ebert-Stiftung, 2012). This is consistent with studies elsewhere (Lee et al., 2016), moreover this study also noted a positive significant association between urban residence and cost-related unmet need. This confirms that place(region) does influence health seeking behaviour, like in this case of unmet need due to cost (Duncan et al., 1993; Vedom \& Cao, 2011).

The results show the positive and negative factors that influence cost-related unmet need controlling for county level variations. Age of household head increased the likelihood of cost-related unmet need for healthcare. This is possibly because older heads are reported to utilize alternative services like self-medication and traditional care (Omonona et al., 2015). An older age of the head of the household has been reported to increase healthcare spending (Kwakye, 2017), resulting in unmet need for care due to high costs. This is in accordance with inequalities in health status that younger people are in general healthier than older people (McMurdo, 2000). Furthermore, some studies have shown that households headed by older people are more likely to experience higher health financial burden (Xu et al., 2003a).

Households with an educated head were less likely to forgo care due to cost. Furthermore, lack of formal education is seen as a predictor of poverty given education opens up a range of income-generating opportunities (J. O. Awiti, 2014). Other studies have also reported lower levels of education increases the odds of having unmet need (Kim et al., 2013).

As expected, the need for inpatient services was associated with a higher risk of cost-related unmet need for care relative to outpatient services. This is because inpatient services are required for more severe illnesses (Atake \& Amendah, 2018), hence demanding high costs of care. Moreover, people may have spent more money for consultation prior to needing inpatient services hence depleted their only savings. Compared with other countries in the region (Ghana, Uganda, Zambia), Kenya is reported 
to have the highest average cost per inpatient bed- day, at about (\$41) per day (IHME, 2014), which hinder more people from utilizing the services.

Good self-rated health was associated with a less likelihood of unmet need for healthcare due to cost. This is consistent with other studies that report those with fair, good or very good self-reported health have a lower likelihood of foregone care, which decreases with better health status (X. Li et al., 2018). People with poor self-rated health are reported to frequent use of medical services (Isaac et al., 2015). Consistent with other studies, those from insured households had decreased odds of unmet need for care due to costs relative to uninsured households. Social healthcare insurance is reported to significantly reduce foregone care in outpatient and inpatient situations (X. $\mathrm{Li}$ et al., 2018). Elsewhere, financial risk protection through insurance is reported to minimize the prevalence of unmet need due to cost (Thammatacharee et al., 2012).

The larger the household, the less likely they are to forgo care due to cost this is perhaps due to reported pooling of resources among the many members as discussed in Chapter 2 which is common in the African context. Despite the analysis showing more rural households (70.3\%) compared to urban households had cost-related unmet need for care, urban households had a positive association with cost-related unmet need. This is corroborated in another study in Kenya that established that forgone care is more heavily concentrated in urban areas (Zollmann \& Ravishankar, 2016) especially within the lowest income quintile. Furthermore in Kenya, rural households are reported to have significantly lower OOPs per capita compared to urban households (Chuma \& Okungu, 2011). There is also greater use of expensive healthcare services, and higher costs charged in urban areas relative to rural areas (Chuma et al., 2007). Additionally, a significant proportion of urban residents (60\% to $80 \%)$ in Kenya live in informal settlements where a substantial proportion face $\mathrm{CHE}$, and are likely to forgo needed healthcare because they cannot afford (Buigut et al., 2015). In many cases, urban households are seen to spend more on OOP for healthcare than rural households, in absolute terms (L. Wang et al., 2016).

Consistent with another study in Kenya that foregone care was related to some extent to the available financial resources in a household (Bonfrer \& Gustafsson-Wright, 2017), our findings show a negative association between household wealth and costrelated unmet need. Economic issues are reported to be the primary reason for not seeking healthcare (Ro et al., 2017). Elsewhere, studies have shown that unmet needs for healthcare due to cost are consistently higher among people in low income groups compared with those in high income groups (Hwang, 2018; OECD, 2017). This implies that wealthy households are less likely to forgo care due to cost, given when prices are high, the poor are more likely than the non-poor to forgo healthcare services (Castro-Leal et al., 2000).

Controlling for the county level significantly improved the model relative to fitting the general logistic regression. An important note we draw from fitting the multilevel model is the existence of disparities in cost-related unmet need between and within the 
counties. It is further evident that there exists variation across the regions that could be substantial in explaining the effect of the individual factors on cost-related unmet need. This implies that regional differences are important in explaining health access due to cost, furthermore the counties have varying socio-economic profiles that could predispose the populations to certain health seeking behaviour. The fact that inpatient services was associated with a higher likelihood of cost-related unmet need, highlights the nuances in measuring OOP payments. This shows that OOP for inpatient services as a fraction of household budget could be underestimated as many people may not seek the services due to cost barriers.

There are limitations to this study that need to be taken into account while interpreting the findings. First, we use the study population as those who reported to have been ill in the 4 weeks preceding the survey and/or needed to be admitted in the past one year. There could be differences in the ability of respondents to report illness or interpret illness differently across population groups. For instance, some people only report serious illness and not what they perceive as "less" serious. Second, the outpatient services focus on only the 4 weeks preceding the survey which could fail to capture some segments of the population given the time of fielding the study. This is because some leading causes of illnesses like Malaria are seasonal, that is they are prevalent at particular times of the year and in certain regions. Third, our analysis is based on a subjective unmet need assessment, i.e., the individuals' self-reported reasons for not seeking care. Previous evidence suggests that perception of health status varies by individual characteristics in that some individuals are better able to estimate their health and their healthcare needs than others. This study relies on the assumption that the response provided by the respondent was the main reason why care was not sought when needed, and hence could be biased because of the subjective interpretation.

\subsection{Conclusions}

This study underscores that affordability is a serious issue in access to healthcare services. The findings suggest that there are multiple factors that drive unmet need of healthcare services due to cost. This requires a multifaceted approach to addressing inequities in unmet need for care, especially among the most vulnerable and marginalised populations. For instance, under the 2010 constitution Kenya setup an 'equalization fund' to address unequal distribution of resources with counties as the unit of distribution. The equalization fund employs a county development index to allocate the funds using four parameters namely, poverty, infrastructure, health and education, to measure need. However, the indicators used to measure access to health are specific to certain services that is maternal health, immunization, and sanitation. This study emphasizes the need to reconsider access to health indicators in computing the county development index, to include overall access to healthcare services thus ensure 'fair' distribution of resources and address access barriers across the counties. 
Health insurance is seen to lower the likelihood of cost-related unmet need, stressing that providing health insurance to the uninsured is key to enabling access. Scaling up of the social health insurance scheme would fundamentally require further segmentation of the population for better targeting, and to ensure it is tailored to match the needs of the various population segments. The current social health insurance scheme is based on a voluntary mechanism which disadvantages the non-employed and poorest given they cannot afford the 'blanket' $\$ 60$ annual premiums. This calls for the need to consider significant improvements to the current insurance package by possibly subsidising premiums or giving exemptions for the most vulnerable and the poorest to improve equity in access. 


\section{Chapter 5 :}

\section{Impact of Household Shocks on the Utilisation of Healthcare Services in Kenya}

Submitted for Publication as:

Njagi, P., Groot, W., Arsenijevic, J., (2020) Impact of Household Shocks on the Utilisation of Healthcare Services in Kenya. 


\section{Abstract}

Background: Households are vulnerable to risks that could trigger income and asset losses, subsequently affecting their wellbeing. These shocks can limit the capacity to access essential welfare services, especially for poor households that are more exposed and less protected. In countries like Kenya where there are inadequate formal protection mechanisms, shocks may drive households into poverty. While research has explored the impact of health shocks on the economic status of the household, there is limited evidence on the effect of assets and income shocks on the utilisation of healthcare services.

Methods: Using the cross-sectional Kenya household budget survey 2015/16, this study examines the effects of household shocks on the utilisation of healthcare services. The analysis used propensity score matching approach to construct a quasi-experimental design adjusting for selection bias of households that experienced shocks.

Results: The results indicate that shocks reduced the probability of utilising healthcare services when households are confronted with an illness. We observed that multiple shocks in a household exacerbate the risk of not utilising healthcare services. Asset shocks had a significant negative effect on the utilisation of healthcare services, whereas the effect of income shocks was not statistically significant. This is presumably due to the smoothing out of income shocks through the sale of assets or borrowing. However, considering the time when the shock occurred, we observed mixed results that varied by the type of shock.

Conclusions: The findings suggest that shocks can limit the capacity of households to invest in healthcare services, emphasising their vulnerability to risks and inability to cope with the consequences. These results provoke a debate on the causal pathway of household economic shocks and health-seeking behaviour. The results suggest a need for social protection programs to integrate mechanisms that enable households to build resilience to shocks. A more viable approach would be to expedite universal health insurance to cushion households from forgoing needed healthcare when confronted with unanticipated risks. 


\subsection{Introduction}

Chapter 4 shows that financial barriers in access to healthcare are more profound for economically disadvantaged households. Poor households are disproportionately at risk of catastrophic expenditure due to OOP payments relative to their well-off counterparts as discussed in chapter 3 . In essence, poor health and the inability to access healthcare are key factors leading to and resulting from poverty(Gray \& Gash, 2016). Although globally, poverty has reduced over time, the majority of the population in developing countries remain poor and susceptible to poverty due to natural and economic shocks (World Bank, 2013). Shocks are defined as adverse events that trigger a decline in well-being, leading to loss of household income, reduction in consumption, and loss of productive assets (D. Clarke \& Dercon, 2009; World Bank, 2000). They are classified into idiosyncratic shocks, referring to those which affect individuals or households, and covariant shocks, referring to those which affect many households, entire communities or regions (Pradhan \& Mukherjee, 2018).

The vulnerability of the population to shocks is among the factors that have constrained poverty reduction in Kenya (Diwakar \& Shepherd, 2018). Currently, around 36 per cent of the population live on less than KES 134 (US\$1.34) per day (Chhabra et al., 2018). A shock can push an already poor-income household into further poverty or drive a non-poor household below the poverty line (Hoogeveen et al., 2005). Households, even those with a relatively higher income, can be disrupted by a financial setback. Financial shocks due to unforeseen expenses and income losses may cause immediate strain, making it difficult to build or rebuild a financial cushion (PEW, 2015). More than $60 \%$ of households in Sub-Saharan Africa (SSA) are reported to have experienced sudden losses in income and assets (Nikoloski et al., 2017). in Kenya, three in every five households have experienced some form of negative shocks, with the majority of households reporting severe shocks of a significant rise in food prices, droughts and floods with a higher incidence in rural areas relative to urban areas (KNBS, 2018a). These shocks are mainly due to Kenya's high exposure to extreme weather patterns, with frequent droughts and floods occurring every three to four years (Parry et al., 2012).

Severe shocks are reported to cause significant indirect effects on health and disproportionately impact the poorest and most vulnerable (L. Clarke \& Masson, 2017). Negative shocks are shown to have an effect on healthcare access for households with limited or no financial protection (Baulia, 2018). When confronted by economic shocks, households' decisions regarding healthcare use become far more discretionary and complex, given they are faced with dilemmas about spending their limited resources (Monheit, 2014). Developing countries are challenged with implementing mechanisms that ensure equitable access to effective health interventions and protect their citizens against health and income shocks (Gottret \& Schieber, 2006). Costs of illness can be substantial when people are not financially protected (WHO, 2007), and this is further aggravated by shocks. 
In Kenya, formal mechanisms such as health insurance and access to formal credit that protect households against the financial consequences of shocks are largely absent, especially among poor rural households (Bonfrer \& Gustafsson-Wright, 2017). Even higher-income households are not fully insured against shocks hence can suffer severe income shortfalls (Pradhan \& Mukherjee, 2018). This suggests that economic shocks are likely to worsen the risks of forgoing care when needed in high- and lowincome households. For instance, loss of income has been associated with a higher risk of unmet healthcare needs (Cheng, 2015; Huang et al., 2014).

The Kenya health care system comprises the public system, with major players including the Ministry of Health( $\mathrm{MOH})$; and the private sector, including private for-profit, NGO, and FBO facilities(NCAPD et al., 2014). In 2013, the public healthcare system was decentralised to new sub-national units (counties) to improve access and service delivery to the people (Williamson \& Mulaki, 2015). The national level is responsible for policy formulation and national referral hospitals that provide sophisticated healthcare services (NCAPD et al., 2011). While the county government is responsible for service delivery at community, primary and country levels( $\mathrm{MOH}, 2014 \mathrm{~b})$.

The provision of healthcare in Kenya is financed through government expenditure, out of pocket (OOP) expenditure and development partners(Chuma et al., 2012; Munge \& Briggs, 2014). OOP expenditure constitutes a significant proportion of health funding in Kenya, at 32 per cent of the total health budget (N. David \& Wanjala, 2020). This spending is due to expenditures at the points of care, which creates financial barriers that threaten the financial security of the households (Dutta et al., 2018). Health payments that are financed out of existing income may lead some households to poverty while others may forgo treatment due to a lack of financial resources (Amponsah, 2016). To date, only about a fifth of the households in Kenya have some form of health insurance (KNBS, 2018a). As a result of limited financial protection, the incidence of catastrophic health expenditure has increased from 6.5\% in 2013 (E. Barasa et al., 2017 ) to $7.1 \%$ in 2018 , with more than one million people pushed into poverty due to healthcare payments annually (Salari et al., 2019). Furthermore, 21.4\% of Kenyans report cost as the main barrier preventing them from seeking care when needed $(\mathrm{MOH}$, 2014a).

Despite the challenges associated with cost, there has been an increase in the annual per capita utilisation rate in Kenya from 1.9 in 2003 to 3.1 in 2013; but with marked disparities across socioeconomic groups ( $\mathrm{MOH}, 2014 \mathrm{a})$. For instance, utilisation of healthcare is higher among wealthy individuals relative to less well-off, with a significant disparity in preventive and inpatient care (Ilinca et al., 2019). Furthermore, other studies have noted that economic reasons influence the demand for modern health care services in Kenya. The poor are less likely to consult a healthcare provider when sick relative to their non-poor counterparts (J. O. Awiti, 2014; MOH, 2014a).

While there is substantial literature on the impact of health shocks on household economic outcomes (Alam \& Mahal, 2014; Amponsah, 2016; Atake, 2018; Adam 
Wagstaff, 2005), there is limited evidence on how economic shocks interplay with the utilisation of healthcare at the household level, particularly in low- and middle-income countries. We identified several studies that have assessed the effects of economic crisis at a macro level on healthcare needs (Baumbach \& Gulis, 2014; Huang et al., 2014; Lusardi et al., 2010; Yang, 2001); however, most have been conducted in high-income countries. Understanding how financial shocks constrain a household's decisions to seek healthcare is paramount in providing policymakers with the evidence to devise mechanisms that cushion the population from financial risks. This study explores how shocks affect households' health-seeking behaviour, considering the timing and type of shock. The study, therefore, responds to the following question: what is the impact of household shocks on the utilisation of healthcare services in Kenya?

\subsection{Methods}

\subsubsection{Data source and sample}

The data used for this study was drawn from the 2015/16 Kenya Integrated Household Budget Survey (KIHBS). This was a nationally representative cross-sectional survey consisting of a sample of 24,000 households (41.2\% in urban and $58.8 \%$ in rural) generated from 2,400 clusters (988 in urban and 1,412 in rural areas). The survey collected information on key socioeconomic aspects in the country, including general health characteristics. Also, it collected data on covariant and idiosyncratic shocks that negatively impact the households' welfare in Kenya. The information on shocks includes the type of loss to the household, the unit impacted by the shock (household/community) and how long ago the shock happened. The response rate for the study was $90.7 \%$, i.e., 21,773 households and 92,846 household members drawn from 2,387 clusters.

Our study data was limited to households that experienced shocks (intervention) and those that had not experienced shocks (control) within the 12 months preceding the survey. This sample included 10,232 households from 2,387 clusters with 16,297 household members who had an illness in the last 4 weeks preceding the survey. The unit of analysis was the individuals within the households.

\subsubsection{Study variables}

\section{Outcome variable}

The outcome of interest was 'utilisation of healthcare' which was used as an operational proxy for access to healthcare services (Jacobs et al., 2012). This was derived from the following questions: (i) 'Was [NAME] sick or injured in the last four weeks?' and (ii) 'Did [NAME] consult a health provider on this sickness /injury in the last four weeks?'. The two questions enabled us to construct the variable ' utilisation of healthcare' based on an individual's perceived need for health intervention. 


\section{Treatment variable}

The main independent (treatment) variable of interest was shock derived from the question 'Over the past five years, was your household severely affected negatively by any of the following events?'. This provided details on the actual type of negative shock experienced by the household including climate shocks such as floods, loss of crops, loss of livestock, loss of employment or business income, loss of remittances, rise in food prices, crime, fire, ethnic conflict, death and evictions. Health shocks were not included in this list.

The study further analysed the type of impact of the shock as follows: 'Did [THIS SHOCK] cause a reduction in household income and/or assets?' This question refers to the perceived loss by the household as either loss of assets, loss of income or loss of both income and asset. The focus of this study was shock(s) that were negatively and exclusively associated with income loss, asset loss or both income and asset loss at the household level within the 12-month period preceding the survey.

Data on time in point when the shock happened was equally assessed as 'How long ago did [THIS SHOCK] occur?'. We constructed three-time points, including shocks that had occurred exclusively within 7-12 months, 1-6 months and below one month. Conversely, we considered households that experienced only one shock to reduce multiple shocks bias. For instance, a household with multiple shocks may have experienced the shocks at different periods of time, and the shocks may have negatively affected either its assets or income or both. We construct multiple treatment groups based on the type of shock (all shocks, multiple shocks, one shocks, income shocks, asset shocks, both income and asset shocks) and the time when the shocks occurred (7-12 months, 1-6 months and below one month).

\section{Matching covariates}

The selection of covariates is a critical aspect in matching as it affects the variance and bias. It is argued that variables related only to the outcome should always be included in a propensity score (PS) model to increase the precision of the exposure effect (Brookhart et al., 2006). However, given that propensity scores are intended to reduce confounding, it is recommended to include variables believed to be related to both the treatment and outcome (Garrido et al., 2014).

We considered seven potential confounding covariates between the utilisation of healthcare services and shocks. The variables considered are structured according to the healthcare utilisation model (Ronald M. Andersen, 1995), and the variables have been discussed as factors influencing healthcare access in Kenya in chapter 4 and other studies (Ilinca et al., 2019). They include the predisposing factors such as 'household head characteristics' (age, gender, education), and enabling factors such as 'household location of residence', 'household size,' 'health insurance status' and 'household economic status '. 


\subsubsection{Propensity score matching approach}

In the absence of a randomised controlled experiment to assess the impact of shocks, we used the potential of observational data and econometric techniques to optimally achieve randomisation. This study used cross-sectional data and employed the propensity score matching (PSM) approach to create a counterfactual to compare the effects between households that have (treatment) and have not (control) experienced a shock. PSM is the conditional probability of assignment to a particular treatment given a vector of observed covariates (Rosenbaum \& Rubin, 1983). It is an alternative method to estimate the effect of receiving treatment when a random assignment of treatments to subjects is not feasible (Thavaneswaran, 2008). There has been growing interest in the use of PSM to estimate the effects of treatments on outcomes using observational studies (Austin, 2011). PSM is argued to correct for selection bias, which is the predominant threat to the validity of using nonexperimental data for impact analyses (Zhehui Luo et al., 2010).

This study implemented PSM by first running a logit model with all the covariates. This estimated the propensity scores(PS) as the probability of experiencing a shock given the confounding factors. The PS were then used to match individuals from households that experienced a shock and those that did not experience a shock given the same likelihood. The outcome of those that experienced shocks was compared with the outcome of a group comparable in observational characteristics but without shocks. This estimated the effect of the shock on the utilisation of healthcare services, and is referred to as the average treatment effect on the treated (ATT).

The matching was implemented using the Stata command 'teffects psmatch' which takes into account the fact that propensity scores are estimated rather than known when calculating standard errors (University of Wisconsin, 2015). We estimated the PS using the one-to-one nearest neighbour matching, with a calliper of 0.1. The 1:1 approach specifies that each individual in the treatment(shocks) is matched with at least one individual from the control (no shocks) level (Stuart, 2010). The 0.1 calliper specifies the maximum distance for which two observations are potential neighbours (StataCorp, 2013). Studies have recommended using calliper of a width equal to 0.2 of the standard deviation of the logit of the PS (Austin, 2010). It is argued that a tighter calliper can improve the performance of the PSM by reducing the bias further and hence lead to closer matches (Lunt, 2014). Therefore, a calliper of less than 0.2 such as in this study is considered acceptable.

\subsubsection{Sensitivity analysis}

For some robustness checks of the results, we conducted sensitivity analyses post matching. The checks were run for all the treatment types including all shocks, multiple shocks, one shock, asset shocks, income shocks and both asset and income shocks. Matching is based on the assumption of unconfoundedness; sensitivity analysis, is, therefore, useful to assess how robust associations are to potential uncontrolled confounding (VanderWeele \& Ding, 2017). This study used the Mantel-Haenszel (MH) 
test statistic referred to as 'MH bounds' proposed in several studies for binary outcomes, (Aakvik, 2001) to check the sensitivity of the results with respect to deviations from the assumption of unconfoundedness (Becker \& Caliendo, 2007). MH bounds were used to test for the conditional independence assumption (hidden bias), which may lead to an overestimation or underestimation of the treatment effect. The $\mathrm{MH}$ bound gamma coefficient shows the factor by which the unobserved factors would affect the selection of households with shocks relative to those without shocks but with similar characteristics.

Another assumption was the sufficient overlap in characteristics to ensure adequate matches. Thus, the quality of the PS model should be assessed based on the covariate balance achieved (Ali et al., 2019). To assess the balance of covariates, we used the 'tebalance' command to check the variance after matching (StataCorp, 2019). Similarly, we constructed density plots to observe how the densities for treatment and controls differed before and after matching. The sensitivity analysis results are discussed later in this chapter.

\subsection{Results}

\subsubsection{Description statistics of the study sample}

Table 5.1 summarises the study sample characteristics by utilisation of healthcare services within the last four weeks preceding the survey. Overall, the eligible sample for this study was 16,297 participants of which those who utilised healthcare services were $13,349(81.9 \%)$, while 2,948 (18.1\%) did not utilise healthcare services. Of the sample, $11,274(69.2 \%)$ had and $5,023(30.8 \%)$ had not experienced shocks in the household within the 12 months preceding the survey. The majority of individuals belonged to male-headed households and were aged between 25-40 years. Additionally, the majority of the households had heads with at least primary level education. We noted that the majority of the sample population were from households above the poverty line and medium-sized households. Lastly, the majority of the study individuals were from uninsured households and resided in rural areas. 
Table 5.1: Descriptive characteristics of the study sample

\begin{tabular}{|c|c|c|c|c|c|c|c|}
\hline \multirow{2}{*}{ Characteristics } & \multirow{2}{*}{ Categories } & \multirow{2}{*}{$\begin{array}{l}\begin{array}{l}\text { Utilised } \\
\text { services }\end{array} \\
\%\end{array}$} & \multirow{2}{*}{$\begin{array}{l}\text { healthcare } \\
\mathrm{N}\end{array}$} & \multicolumn{2}{|c|}{$\begin{array}{l}\text { Did not utilise } \\
\text { healthcare services }\end{array}$} & \multicolumn{2}{|l|}{ Total } \\
\hline & & & & $\%$ & $\mathrm{~N}$ & $\%$ & $\mathrm{~N}$ \\
\hline \multirow{2}{*}{$\begin{array}{l}\text { Shock in the } \\
\text { household }\end{array}$} & No shocks & 31.17 & 4161 & 29.24 & 862 & 30.82 & 5023 \\
\hline & Shocks & 68.83 & 9188 & 70.76 & 2086 & 69.18 & 11274 \\
\hline \multirow{2}{*}{$\begin{array}{l}\text { Gender of } \\
\text { household head }\end{array}$} & Male & 65.84 & 8789 & 62.79 & 1851 & 65.29 & 10640 \\
\hline & Female & 34.16 & 4560 & 37.21 & 1097 & 34.71 & 5657 \\
\hline \multirow{3}{*}{$\begin{array}{l}\text { Age group of } \\
\text { household head }\end{array}$} & Below 25 years & 4.22 & 563 & 4.17 & 123 & 4.21 & 686 \\
\hline & $25-40$ years & 51.41 & 6863 & 47.49 & 1400 & 50.7 & 8263 \\
\hline & $40+$ years & 44.37 & 5923 & 48.34 & 1425 & 45.09 & 7348 \\
\hline \multirow{4}{*}{$\begin{array}{l}\text { Education level of } \\
\text { household head* }\end{array}$} & Primary & 50.00 & 6627 & 48.26 & 1415 & 49.68 & 8042 \\
\hline & Secondary & 23.11 & 3063 & 19.54 & 573 & 22.46 & 3636 \\
\hline & Tertiary & 10.02 & 1328 & 7.20 & 211 & 9.51 & 1539 \\
\hline & Others & 16.88 & 2237 & 25.0 & 733 & 18.35 & 2970 \\
\hline \multirow{2}{*}{ Economic status } & Above Poverty line & 66.11 & 8825 & 58.04 & 1711 & 64.65 & 10536 \\
\hline & Below Poverty line & 33.89 & 4524 & 41.96 & 1237 & 35.35 & 5761 \\
\hline \multirow{3}{*}{ Household Size } & 1-3 Small Size & 26.74 & 3570 & 30.87 & 910 & 27.49 & 4480 \\
\hline & 4-6 Medium Size & 47.33 & 6318 & 40.43 & 1192 & 46.08 & 7510 \\
\hline & 7+ Large Size & 25.93 & 3461 & 28.70 & 846 & 26.43 & 4307 \\
\hline \multirow{2}{*}{ Residence } & Rural & 65.11 & 8692 & 65.54 & 1932 & 64.91 & 5673 \\
\hline & Urban & 34.89 & 4657 & 34.46 & 1016 & 35.09 & 10624 \\
\hline \multirow{2}{*}{$\begin{array}{l}\text { Health insurance } \\
\text { status** }\end{array}$} & Insured & 18.81 & 2509 & 11.33 & 334 & 17.45 & 2948 \\
\hline & Not insured & 81.19 & 10833 & 88.67 & 2614 & 82.55 & 13342 \\
\hline Total & & 81.91 & 13349 & 18.09 & 2948 & & 16297 \\
\hline
\end{tabular}


Figure 5.1 below presents the percentage of individuals from households that had experienced shocks in the 12 months preceding the survey. The analysis indicates that $11,274(69.2 \%)$ had experienced shocks. Of this, 7,320 (44.9\%) had experienced multiple shocks while 3,954 (24.3\%) had experienced only one shock in the household. Further analyses showed that, of those that had experienced one shock in the household $1,780(45 \%)$ had experienced shocks that led to only income losses, while $550(13.9 \%)$ had experienced shocks that led to only assets losses and 1,073(27.1\%) had experienced shocks that led to losses in both income and assets. Another group of 551 (13.9\%) experienced shocks; however, since they reported the shocks to not have affected either assets or income, we do not include this group in this study. Appendix 5A provides a further description of those individuals from households that had experienced different types of shocks and the time when the shocks happened.

Figure 5.1: Percentage of individuals who experienced shocks in the household

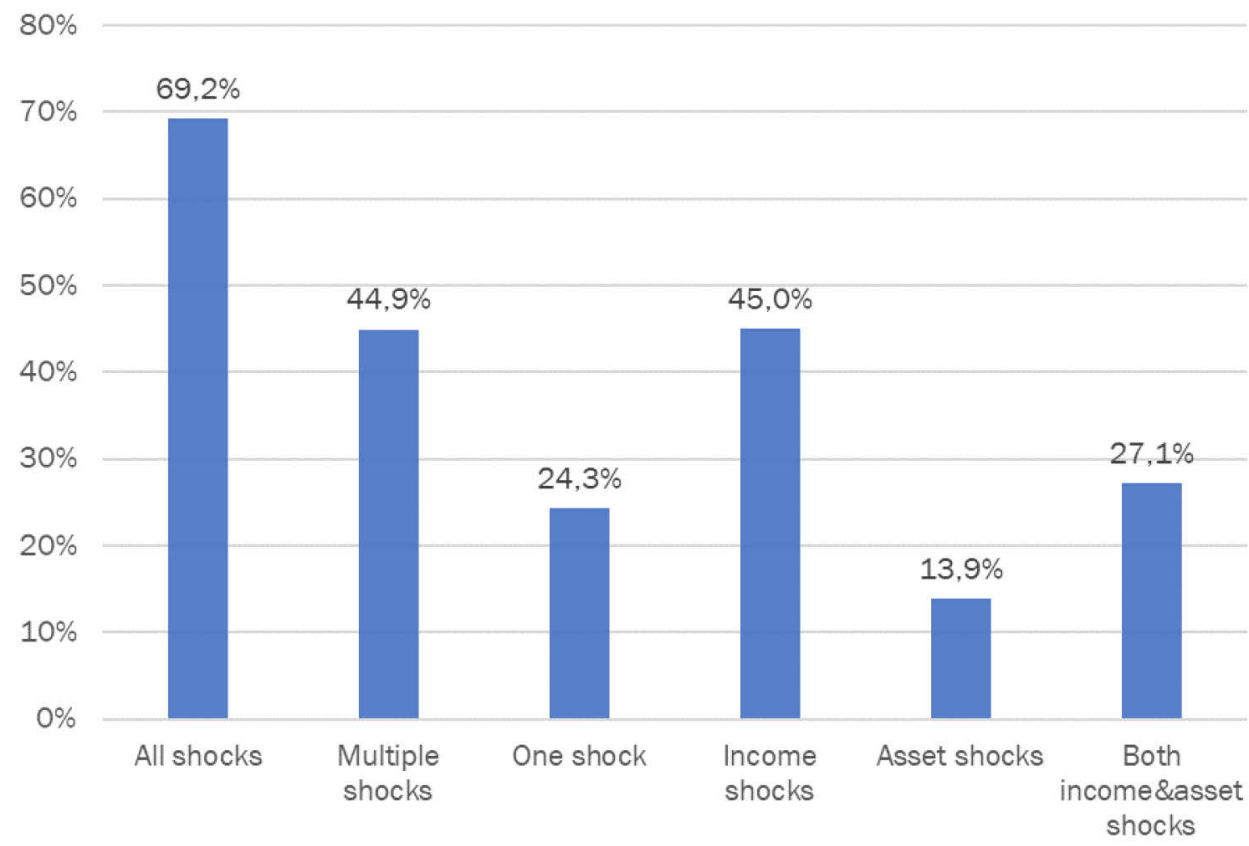




\subsubsection{Effect of shocks on the utilisation of healthcare services}

Table 5.2 presents the mean differences between the households which did and did not experience shocks as well as the estimated effects of the shocks on the matched samples. After adjusting for systematic differences in the observed characteristics of households with shocks and those without shocks, the results show a significant negative effect of household shocks on the utilisation of healthcare services. Generally, shocks contributed to a 1.6 percentage point decrease in utilisation of healthcare services. Multiple shocks reduced utilisation of healthcare service by two percentage points.

The assessment of households that experienced only one shock revealed that there was no statistically significant effect on the utilisation of healthcare services. Further analysis of these households was carried out by disaggregating them into exclusively asset shocks, income shocks and both income and asset shocks. Within those that had experienced only one shock, the effect was statistically significant for only asset shocks. Asset shocks contributed to a 4.1 percentage point decrease in the utilisation of healthcare services.

Table 5.2: Average treatment effect on the treated (ATT) of shocks on utilisation of healthcare services

\begin{tabular}{|c|c|c|c|c|c|c|c|}
\hline \multirow[b]{2}{*}{ Type of shock } & \multicolumn{2}{|c|}{$\begin{array}{l}\text { Means after } \\
\text { matching }\end{array}$} & \multicolumn{4}{|c|}{ Average treatment effect on the treated(ATT) } & \multirow{2}{*}{$\begin{array}{l}\text { Number of } \\
\text { cases on } \\
\text { support (N) }\end{array}$} \\
\hline & Treated & Control & ATT & SE. & T-stat & {$[95 \% \mathrm{Cl}]$} & \\
\hline All Shocks & 0.815 & 0.831 & $-0.016^{* *}$ & 0.007 & -2.11 & {$[-0.030--0.001]$} & 16290 \\
\hline Multiple shock & 0.811 & 0.831 & $-0.020 * *$ & 0.008 & -2.41 & {$[-0.035--0.004]$} & 12338 \\
\hline One shocks & 0.821 & 0.830 & -0.008 & 0.009 & -0.93 & {$[-0.026--0.009]$} & 8972 \\
\hline Asset shocks & 0.785 & 0.827 & $-0.041 * *$ & 0.020 & -2.03 & {$[-0.081--0.001]$} & 5570 \\
\hline Income shocks & 0.847 & 0.829 & 0.018 & 0.011 & 1.66 & {$[-0.003-0.040]$} & 6799 \\
\hline $\begin{array}{l}\text { Both asset \& } \\
\text { income shocks }\end{array}$ & 0.806 & 0.825 & -0.019 & 0.014 & -1.32 & {$[-0.047--0.009]$} & 6092 \\
\hline
\end{tabular}

\subsubsection{Effect of the type and time of shock on utilisation of healthcare services}

Analysis of the effect by type of shock and the time when the shock occurred is illustrated in table 5.3. The findings indicate that there was a significant negative effect on the utilisation of healthcare services for asset shocks that occurred between 7-12 months. Asset shocks within 7-12 months contributed to a 9.7 percentage point decrease in the utilisation of healthcare services. On the contrary, there was a significant positive effect on the utilisation of healthcare services for income shocks that had occurred between 1-6 months. The results indicate that income shocks within 1-6 
months contributed to a 3.5 percentage point increase in the utilisation of healthcare services.

Shocks that occurred within less than one month happened within the same time illness was reported. A significant negative effect was observed on the utilisation of healthcare services for shocks that affected both income and assets in below one month. The results indicate a 6.7 percentage point decrease in the utilisation of healthcare services for households that experienced both income and asset shocks.

Table 5.3: Average treatment effect on the treated (ATT) by time of shock on utilisation of healthcare services

\begin{tabular}{|c|c|c|c|c|c|c|c|}
\hline \multirow[t]{2}{*}{$\begin{array}{l}\text { Type and time of } \\
\text { shock }\end{array}$} & \multicolumn{2}{|c|}{$\begin{array}{l}\text { Means after } \\
\text { matching }\end{array}$} & \multicolumn{4}{|c|}{ Average treatment effect on the treated(ATT) } & \multirow{2}{*}{$\begin{array}{l}\text { Number } \\
\text { of cases } \\
\text { on } \\
\text { support } \\
\text { (N) }\end{array}$} \\
\hline & Treated & Control & ATT & S.E. & T-stat & {$[95 \% \mathrm{Cl}]$} & \\
\hline \multicolumn{8}{|l|}{ Asset shocks } \\
\hline 7-12 months & 0.739 & 0.836 & $-0.097 * *$ & 0.048 & -2.02 & {$[-0.191--0.003]$} & 5112 \\
\hline $1-6$ months & 0.815 & 0.828 & -0.014 & 0.024 & -0.56 & {$[-0.062-0.034]$} & 5322 \\
\hline Below 1 month & 0.756 & 0.814 & -0.057 & 0.041 & -1.39 & {$[-0.138-0.024]$} & 5176 \\
\hline \multicolumn{8}{|l|}{ Income shocks } \\
\hline 7-12 months & 0.812 & 0.824 & -0.013 & 0.028 & -0.45 & {$[-0.067-0.042]$} & 5275 \\
\hline $1-6$ months & 0.863 & 0.828 & $0.035 * *$ & 0.014 & 2.46 & {$[0.007-0.063]$} & 5946 \\
\hline Below 1 month & 0.838 & 0.835 & 0.003 & 0.017 & 0.16 & {$[-0.031-0.037]$} & 5618 \\
\hline \multicolumn{8}{|c|}{ Income \& Asset shocks } \\
\hline 7-12 months & 0.863 & 0.836 & 0.026 & 0.034 & 0.78 & {$[-0.040-0.093]$} & 5195 \\
\hline $1-6$ months & 0.813 & 0.824 & -0.011 & 0.018 & -0.60 & {$[-0.046-0.024]$} & 5625 \\
\hline Below 1 month & 0.757 & 0.824 & $-0.067 * * *$ & 0.026 & -2.54 & {$[-0.119--0.015]$} & 5312 \\
\hline
\end{tabular}

\subsubsection{Sensitivity analysis results}

The results of the $\mathrm{MH}$ sensitivity analysis are reported in Appendix 5-B. If there is no hidden bias, the gamma coefficient $(\Gamma=1)$ is similar to the ATT estimates. The test statistic (at $\Gamma=1$; the assumption of no hidden bias) for the overall shocks and multiple shocks gave similar results to the ATT estimates suggesting no hidden bias. Checks for the treatment effect by type of shocks (one shock, income shocks, assets shocks) indicate varying critical values at $\Gamma=1$ at which the $p$-level of the estimated treatment effect is statistically significant, potentially implying sensitivity to unobserved heterogeneity, which would overestimate the true treatment effect. 
Second, the results of the balance between covariate tests show that matching improved the level of balance given the standardised differences for all covariates categories are close to zero (0), and the variances are close to one (1). The results of the 'tebalance' checks are presented in Appendix 5-C.

Last, all the density plots show that the matching is balanced between the control and treatment groups as the curves perfectly overlay after matching. Below, we have provided a graph of the density plots for overall shocks before and after matching. All the density plots for the other treatment(s) that is; type of shocks and time when the shocks occurred are presented in Appendix 5-D.

Figure 5.2: Density plots for all shocks before and after matching

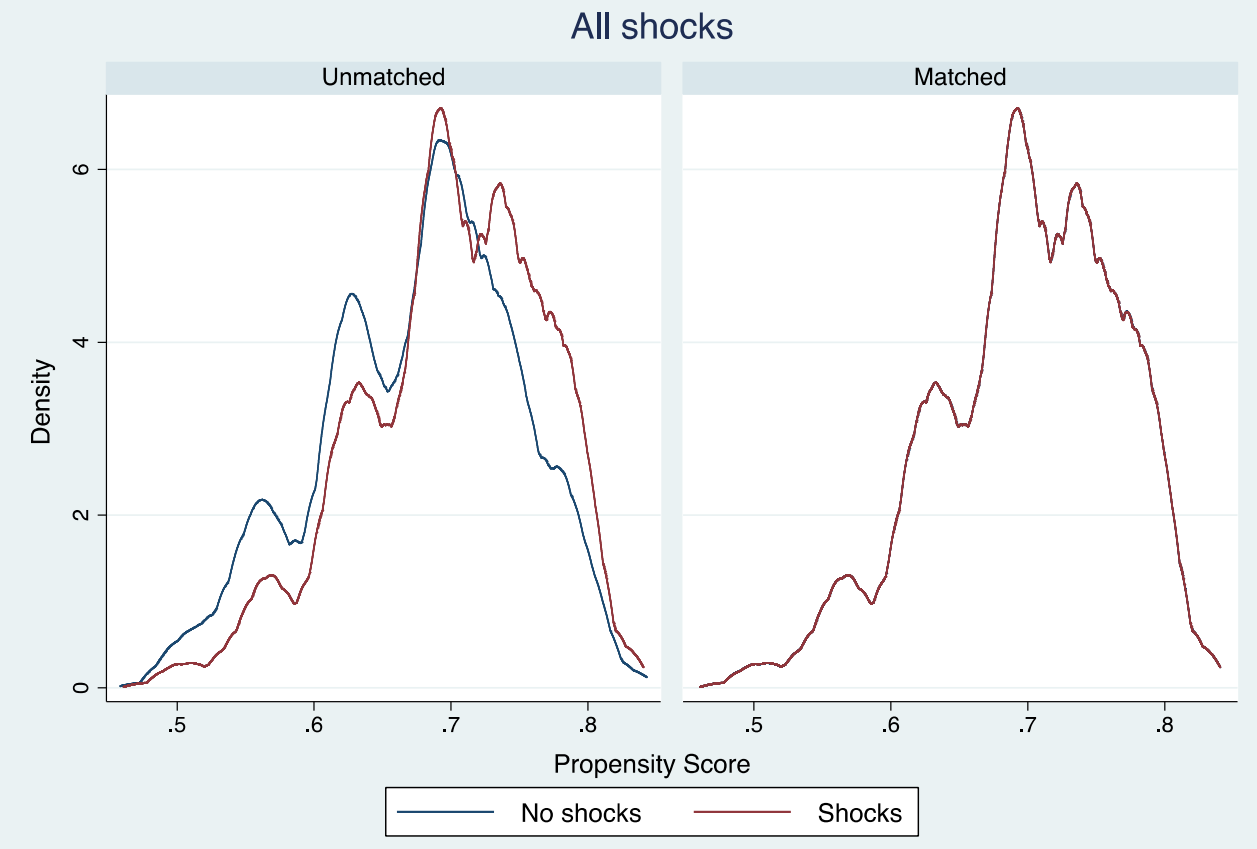

\subsection{Discussion}

This study estimated the impact of shocks on the ability to utilise healthcare services using the PSM approach. The findings show that overall shocks have a significant negative effect on the utilisation of healthcare services after matching on all confounding covariates. This is consistent with other studies that have shown households in economic distress (wealth and income losses) were more likely to reduce medical care usage (Lusardi et al., 2010). Furthermore, as evidenced in Chapter 4, in Kenya high cost of care and lack of money are key barriers to accessing healthcare services. In accordance with other studies elsewhere (Mazumdar et al., 2014), the results show that multiple shocks 
exacerbate the risk of non-utilisation of healthcare services by increasing the number of adverse welfare outcomes, thus making a recovery and coping much more difficult. Evidence suggests that consumption smoothing is more challenging with repeated shocks because households may deplete their assets, limiting their ability to cope with subsequent shocks; besides, one shock may lead to another (World Bank, 2000). Due to the complex and extreme weather patterns in Kenya, households are vulnerable to multiple climatic shocks (Parry et al., 2012). Moreover, the descriptive analysis indicates that the majority of the households were faced with multiple shocks within the 12 months preceding the survey.

We interpreted the effect results of type of shocks on the utilisation of healthcare services with caution, given that the $\mathrm{MH}$ bounds sensitivity checks point to the presence of unobserved heterogeneity which possibly overestimated the true treatment effect. This insinuates that households that are likely to experience asset and income shocks tend to have higher non-utilisation rates even in the absence of shocks. In general, income shocks had no effect on the utilisation of healthcare services. However, considering when the shock occurred, the results indicate a significant positive effect of income shocks on the utilisation of healthcare services in 1-6 months. This could be attributed to the smoothing out of the income shocks by selling assets or borrowing (McPeak, 2004). Other studies corroborate these findings; for example, a study in neighbouring Uganda showed that negative income shocks increased the probability of uptake of healthcare services for children, such as the provision of Vitamin $A$ supplementation. The buffer stock mechanism was argued to smooth out the shock, allowing for time investment in health promotion for children (Baulia, 2018). Additionally, income shocks could be transitory, and hence the households may have recovered from the shock at the time of illness. Evidence suggests that households with a sufficiently high level of assets are not only able to fully offset transitory income shocks (Beegle et al., 2006) but also insure against permanent income shocks to some degree (Fella et al., 2017).

Overall, households that experienced asset shocks are less likely to utilise healthcare services when faced with an illness. Considering the time when the asset shocks occurred, the results indicate that a negative effect was observed for asset shocks in 7-12 months. This is perhaps because asset shocks are more permanent with longer-term impact; hence, households may take longer to recover. Furthermore, households consider asset loss to be a more severe shock, and more often in rural areas, the highest percentage of shocks results in asset loss (Doss et al., 2015). Besides, many rural households in SSA countries rely on informal mechanisms such as savings, reduction of household expenditure and even sale of assets to cope with shocks due to the less effective financial markets (Christiaensen \& Demery, 2017). Since rural populations rely on agricultural produce and livestock to accumulate assets (Bettencourt et al., 2015; Rapsomanikis, 2015), asset losses could cause a severe negative impact on the household's welfare. Besides, livestock and crop-related losses have been reported to lead households into poverty in Kenya (Kristjanson et al., 2010). Poor 
households could become locked into a state of few or negligible assets when susceptible to the worst impacts of different types of shocks and thus become stuck in a poverty trap (Mendoza, 2009).

We note that shocks that led to a loss of both income and assets in below one month had a significant negative effect on utilisation of healthcare services. Intuitively, shocks that happen within the time of illness are expected to have a more profound effect on a financially constrained household's decision to seek care. However, given the nature of the data utilised, we could not establish if the shock happened prior to or after the illness. Furthermore, shocks were subjectively defined by households; hence the value and impact of the shock vary according to the household economic status(TranQuang, 2012). This suggests that a shock of lower value for a poor household could mean a loss of everything the household owns. In contrast, for well-off households, a high-value loss could be a small proportion of the household wealth, and the household may have alternative assets or savings. This is a reflection of the multifaceted nature of shocks (Nikoloski et al., 2017).

Overall, we noted that shocks have an effect on households' health-seeking behaviour; however, this effect varies according to the timing and type of the shock. Nevertheless, shocks may leave no profound impact on households' economic wellbeing, given that the adverse impacts of shocks could fade over time due to labour and commodity market adjustments (Akter \& Basher, 2014).

In Kenya, climatic shocks happen in tandem with the emergence and spread of some infectious diseases. For instance, during heavy rains that cause floods, diseases such as cholera are bound to spread, whereas, during hot weather when droughts occur, diseases such as malaria are prevalent (National Research Council, 2001). This could result in a double burden given the loss of assets or income, which happens at the same time with the need to access healthcare services, making it arduous for households to cope.

Kenya has made significant progress in building nationally owned social protection systems. However, these have been targeted at specific groups of the population, including persons with disabilities, orphans, the elderly and those in foodinsecure areas (GOK, 2017a). Other systems such as the social pension fund and health insurance are voluntary - based on an individual's ability to pay (E. Barasa et al., 2018). This limits coverage for the majority and especially the poor who do not fall into the specified groups. Considering that three out of every five households have experienced a shock, this could mean that thousands of households are at the risk of forgoing the needed healthcare due to risks. Moreover, the majority of the population in Kenya does not possess any form of healthcare insurance.

This study has some limitations that need to be considered while interpreting the findings. First, while this study assumes a direct interaction with income and asset shocks to provide estimates of the effects, health-seeking behaviour is much more complex. Second, we applied PSM, a quasi-experimental approach that removes bias 
due to observed characteristics but not necessarily the unobserved characteristics. It is, therefore, possible that bias could arise from the variable omission and unobserved heterogeneity. Nonetheless, we used all possible observable characteristics evidenced in the literature as confounders and equally ran the sensitivity analysis to check for hidden bias. Furthermore, panel data is more robust and recommended for this type of impact analysis. Third, the utilisation of healthcare services is based on four weeks preceding the survey. Therefore, for the shocks that had occurred in below one month, we could not discern if they happened prior to or after the illness. Additionally, shocks in the survey were subjectively defined and self-reported depending on the household's interpretation of asset and income losses which can vary.

\subsection{Conclusion}

The adjusted estimates demonstrate that shocks limit the capacity of households to afford and access healthcare services when needed. The effects of shocks on the utilisation of healthcare services are dependent on the type of shock and the time when the shock occurred. This fundamentally implies that in countries such as Kenya, where financial protection mechanisms are limited, households adjust their health-seeking behaviour when confronted with resource constraints in times of illness.

The findings stress the vulnerability of households to risks and their inability to cope with the consequences. This should provoke debates on the interaction and causal pathway of the households' economic shocks and health-seeking behaviour. In addition, it calls for the broadening of government social protection programs to integrate mechanisms that enable households to build resilience to shocks. A more viable approach would be to expedite the expansion of the health insurance scheme(s) to guarantee affordability and accessibility to healthcare services for all. 
Chapter 6 :

Conclusion and Policy Implications 


\subsection{Introduction}

This dissertation was inspired by the concerns about equity of access to healthcare, which is one of the key drivers to the achievement of UHC. The main purpose of this dissertation was to examine equity of access to healthcare services, focussing on cost and affordability as an 'entry' to the healthcare system. It explored the nuances in the measurement of and factors that drive inequities in access to healthcare services in Kenya. UHC is a priority in Kenya, with the principal focus of the health policy 2013-2030 being to ensure equity and people- centredness. This then begs the question: how best to ensure equity of access to healthcare services, while guaranteeing adequate protection for the most vulnerable sections against the financial costs of ill health?

There has been limited evidence on the extent of equity of access in Kenya, and the SSA region. Even where evidence is available, it focuses on only one perspective of equity of access such as CHE (E. Barasa et al., 2017; Chuma \& Maina, 2012; Salari et al., 2019). This dissertation adds to the existing evidence by exploring more than one perspective of equity in access to healthcare services and the associated drivers. This includes the measures to assess the socioeconomic inequalities in $\mathrm{CHE}$, the cost-related unmet needs, and the effects of households' economic shocks on the utilisation of healthcare services in Kenya.

This dissertation focused on four key questions discussed in the various chapters, addressing the main aim - achieving equity of access. Chapter 2 examined the extent of catastrophic health expenditure in SSA and the associated determinants. The analyses point to evidence of existing variations in financial risk protection, based on the diverse contexts of the healthcare systems in the SSA countries. It identifies the drivers of CHE, which are largely similar across various geographical settings. Chapter 3 moves beyond the measures of financial risk protection to analyse the socioeconomic inequalities in $\mathrm{CHE}$, the factors that sustain the inequalities and the changes over time. The results illustrate that despite the declining incidence of catastrophic health expenditure, propoor inequalities in CHE have increased over time and that this is attributable to the economic status of the households. Besides, the results point to the worsening of catastrophic payments amongst the poor, vis-a-vis the well-off. The findings are expected to nudge policymakers to formulate appropriate pro-poor measures aimed at reducing the inequalities over time.

Chapter 4 follows on to examine the extent of unmet need for healthcare services due to cost barriers, accounting for the differentials at the county (sub-national) level. The results showed the existence of cost-related unmet need and the wide disparities across the counties in Kenya. Assessing the county variations in unmet need is useful to inform the prioritisation of healthcare services in the counties that are lagging behind, thus addressing inequities in the country. Finally, Chapter 5 explores how adverse household shocks influence the uptake of healthcare services when illnesses occur. The results point to the role of asset and income shocks in limiting households' ability to invest in healthcare services. The findings are critical in providing insights into the policy 
interventions that not only offers a cushion against financial risks, but also build the financial resilience of households.

\subsection{Discussion of the findings}

The various chapters in this dissertation culminate in key cross-cutting findings. These findings are presented below as five main statements:

\section{The poor are the most vulnerable to catastrophic payments, yet they are the least protected from the financial burden imposed by healthcare costs.}

Populations living in poverty are reported to experience multiple deprivations of income, housing and even essential services such as healthcare and education (Mohanty, 2012). Persons in lower socioeconomic groups have higher rates of morbidity, and consequently a greater need for healthcare services (Chuma \& Okungu, 2011). A consistent finding across the chapters in this dissertation was that households of lower socioeconomic status face the greatest financial hardship due to healthcare payments.

In Chapter 2, the systematic review draws attention to groups that are not financially autonomous and hence more likely to experience catastrophic costs. This is an indication that the vulnerability of low-income groups to financial risks is a common challenge across the SSA countries. Furthermore, for households with low income, even a small amount of healthcare payment can be catastrophic (Masiye et al., 2016). For example, the poorest households in Kenya are reported to spend a third of their resources on healthcare payments each year, compared to only $8 \%$ spent by the richest households (Chuma \& Maina, 2012).

In accordance with the findings from Chapter 2, socioeconomic inequalities in CHE are disproportionately concentrated amongst the poor in Kenya, as shown in Chapter 3. This dissertation shows that although a decline in the overall incidence of CHE was observed, the rich experienced a higher percentage of decline, compared to the poor. The decline in CHE can be partly explained by the '10/20 policy' introduced in 2004, which waived user charges and introduced a uniform registration fee of Kenyan shillings 10 and 20 (\$0.1/\$0.21 -at the current rate). However, studies have found nonadherence to the $10 / 20$ policy, where health providers charged more due to insufficient resources to cover operational costs like support staff and laboratory services (Opwora et al., 2015), which is burdensome to the poor. Moreover, based on this dissertation, results show that there was an increase in the pro-poor inequalities in CHE over time, indicating that the financial risk protection mechanisms in place may have favoured the rich and not the poor. For instance, a study on the $10 / 20$ policy about health facility delivery showed that the policy favoured more the well-off than the poor, with health facility deliveries increasing for the well-off while the poor delivered at home (Obare et al., 2018). These findings led the government to revisit this policy, abolished user fees at dispensaries and health centres and introduced free maternity services for all in 2013. However, healthcare services in hospitals are still offered at a fee, which can still

1) \$ US Dollar = 100 Kenya Shillings; June 2020. 
discourage the poor from accessing care. Recent studies show that CHE has increased from $6.5 \%$ in 2013 to $7.1 \%$ in 2018 , disproportionately concentrated among the worseoff (Salari et al., 2019). The persistent inequalities in Kenya have significantly exacerbated the burden of the poor (KNBS, 2018b) making it even more challenging for them to access healthcare services. This dissertation further shows that it was the elasticities in the wealth status that significantly contributed to the increase over time in the inequality in CHE.

Chapter 4 corroborates that the poor are more likely to forgo healthcare due to lack of money and the high cost of healthcare services. The poor counties are seen to have higher cost-related unmet need, vis-a-vis the well-off counties, resulting in wide variations across the counties. Evidence in Kenya suggests that poverty has adverse effects on an individual's demand for healthcare services (J. O. Awiti, 2014). And even so, shocks can push an already poor household deeper into poverty or drive a non-poor household below the poverty line (Hoogeveen et al., 2005). Poor households are more vulnerable to shocks and hence are faced with difficult choices on spending their limited resources when illnesses occur, prompting them to refrain from seeking healthcare services. This is substantiated in Chapter 5 that shows both asset and income shocks reduce utilisation of healthcare services. In Kenya, the incidence of shocks is higher for households in the poor counties like Kitui (96\%) and Migori (92\%), compared to the welloff counties like Kiambu (16.8\%) and Mombasa (23.6\%) (KNBS, 2018a). Wealthier households have a better financial cushion and have the ability to pay for care and insurance premium, relative to the poor households. Similarly, in Ghana, wealthier populations are reported to be better placed to enrol for health insurance than poorer populations, despite insurance premiums varying by income scale (Blanchet et al., 2012).

In addition, the NHIF, which is the leading national health insurance provider in Kenya, is expected to provide a financial cushion, which is based on a premium; thus, the poor may still lack the ability to pay. Furthermore, disparities exist in insurance coverage, with only $2.9 \%$ of the poorest quintile being insured, compared to $16 \%$ of the richest quintile. Even the community-based insurance covers mostly the middle quintile, while private insurance largely covers the richest quintile at 17 per cent ( $\mathrm{MOH}, 2014 \mathrm{a})$. As a consequence of the limited financial risk protection for the poor and most vulnerable population, annually, more than one million Kenyans are pushed into poverty due to healthcare payments (Salari et al., 2019).

\section{Disparities in financial risk protection allude to the existence of inequities in various levels of the healthcare delivery system.}

WHO emphasises the importance of achieving equity in health and access to healthcare services through actions on the social determinants of health (SDH) (WHO, 2010; Zere \& McIntyre, 2003). The SDH framework views the healthcare system as an intermediary determinant, whose role is relevant for equitable access to care by addressing differences in vulnerability and exposure (WHO, 2010). System-level factors 
may constrain the social and environmental context in which healthcare disparities are produced (Brown et al., 2019), leading to further deterioration of people's social status. For instance, OOP payments can often turn catastrophic or drive households further into poverty. Financial risk protection is concerned with safeguarding people against the financial hardship associated with paying for healthcare services (Saksena et al., 2014), thereby contributing to reducing inequities.

Chapter 2 of this dissertation shows that system-level factors like the type of health provider are associated with the incidence of CHE. For instance, higher CHE was associated with seeking care from private health providers, relative to public health providers, while within the public care system, lower levels of care (primary) were associated with a reducing effect on $\mathrm{CHE}$. Studies have noted significant differences in access to healthcare between public and private providers in Kenya. Evidence suggests that the public sector serves more poor clients relative to the private sector that serves the wealthy in search for better quality services, given their ability to pay (Chakraborty et al., 2017). In addition, significant inequities are observed in the use of healthcare services, especially preventive and inpatient care services (Ilinca et al., 2019).

An inequitable distribution of resources, disparities in access and service delivery continue to be manifest in the Kenya healthcare system. For instance, urban areas are seen to have better access to healthcare services relative to rural areas; yet, healthcare services in urban areas are also of higher quality than those available in rural areas (Mulaki \& Muchiri, 2019). Chapter 4 of this dissertation emphasizes these regional disparities, which can be attributed to structural funding problems, differences in investments and availability of quality healthcare facilities (Dutta et al., 2018). These disparities are partly because counties have autonomy on the allocation and spending of their resources, depending on their priorities. For instance, in 2017, Marakwet county spent $40 \%$ of its total budget allocation on health, compared to the marginalised Mandera county that spent about 15\% (Mulaki \& Muchiri, 2019).

Other system-level disparities include the unequal distribution of healthcare workers, by urban/rural areas, by regions, and by the level of care. Rural dispensaries have 20 percent fill rates of their nursing establishments, while district hospitals have 120 percent fill rates. Further, the doctor-patient ratio is about 1:20,700 in the Central region, as against $1: 120,000$ in the North-Eastern region (Luoma et al., 2010). In addition, there exist variations in the healthcare infrastructure. For instance, while the national average facility density was 2.04 facilities per 10,000 persons in 2014 , the variations across counties ranged between less than 1 facility per 10,000 persons and over 3.5 per 10, 000 population ( $\mathrm{MOH}, 2014 \mathrm{c})$. There exist marked variations in access to health facilities in relation to distance and time taken. More than half of the 47 counties have an average distance to a health facility which is higher than the national average of 3 kilometres. Average distance to health facilities across the counties ranges between a low of 1.4 kilometres and a high of 52.6 kilometres, while the time taken ranges between 13.3 minutes and 93.3 minutes (Mugo et al., 2018). These system 
disparities result in variations in healthcare costs, as confirmed in this dissertation that the differences between the counties accounted for a significant variation in cost-related unmet need. For instance, in rural areas and in counties that are underserved with either inadequate health workers or health facilities people travel long distances to seek better healthcare, resulting in even higher costs.

To address regional disparities, Kenya decentralised its healthcare system in 2013 in line with the new constitution of 2010 (Williamson \& Mulaki, 2015) and established an equalisation fund to support economically marginalised counties to bring them on par with their counterparts. The fund is allocated $1.5 \%$ of the annual national revenue collected, to be used for the provision of essential services, including water, health facilities and electricity in these marginalised areas (GOK, 2010), an indication that unless regional disparities are addressed through the transformation of system-level factors, equity of access cannot be achieved, as some counties will continue to lag behind. To achieve a more sustainable impact on equity in access, it is paramount for the national and county governments to invest in strengthening the healthcare delivery systems. Further, improving demand for healthcare services without commensurate investment in supply factors could reverse the gains achieved at the individual/household level.

\section{While formal health insurance is a necessary and essential factor in improving access to healthcare services in Kenya, it is not sufficient on its own.}

Apart from social health insurance, the government could consider prepayment mechanisms to pool risk across populations (Ezat Wan Puteh \& Almualm, 2017). However, there are debates on risk pooling in LMICs like Kenya, given the majority of people lack any form of health insurance.

One way to protect vulnerable groups from financial burden is to decrease the extent of out-of-pocket payments and provide adequate financial risk protection (Essue et al., 2015). Establishment of social health insurance (SHI) is among the solutions proposed to reduce costs to households when seeking care in various low- and middleincome countries (Acharya et al., 2013). Kenya uses health insurance as one of the mechanisms for financial risk protection, through contributions which are mandatory for the formal sector and voluntary for the informal sector. However, only about $20 \%$ of the population has some form of health insurance. In Kenya, inequalities in health insurance coverage are attributable to voluntary and contributory health insurance mechanisms, based on ability to pay, signifying the inappropriateness of contributory schemes in highly informal and poor settings (Kazungu \& Barasa, 2017). Uptake of insurance could also be associated with individual psycho-social determinants like trust, risk-preferences, and perceived healthcare quality (Adebayo et al., 2015).

Insurance can contribute to lower out-of- pocket costs and even reduce borrowing for healthcare costs (Barnes et al., 2017). Chapter 3 of this dissertation shows that health insurance is associated with a reduction in socioeconomic inequalities related to $\mathrm{CHE}$ and is therefore a crucial aspect of risk protection. Universal health insurance is 
reported to yield better outcomes in providing financial risk protection to larger populations (Skinner et al., 2019), coupled with other socioeconomic measures. Furthermore, even when premiums are relatively low, there are socioeconomic factors that influence the uptake of health insurance (Alesane \& Anang, 2018).

Chapter 2 of this dissertation shows that non-medical costs like those for transport and food markedly contributed to catastrophic payments, compared to direct medical costs, implying that insurance per se is inadequate in cushioning against financial hardships due to healthcare costs. Financial risk protection should therefore extend beyond medical costs, for which formal insurance does not provide sufficient cushion. For instance, populations, especially in rural areas, have to travel long distances, lack public transport, have to face poor road condition and pay high transport costs, which limit their access to healthcare services (Kabia et al., 2019). Decentralisation of the healthcare service delivery to the sub-national county level is one approach through which the Kenyan government aims to improve geographical access and availability of healthcare services (McCollum et al., 2019). Early results from some of the counties show that decentralisation has created opportunities for local-level prioritisation and community involvement in the health sector (Tsofa et al., 2017).

The large informal sector population in Kenya and other countries in the SSA region has limited the taxation capacity of governments (Preker et al., 2007); hence, part of the healthcare financing is through out-of-pocket payments. Mechanisms such as micro health insurance (MHI) have been shown to contribute to government efforts in offering risk protection to the large numbers of informal workers (Kimball et al., 2013). However, even community-based health insurance and voluntary schemes present various risks and challenges that are related to package coverage, amount and timing of premiums, rising healthcare costs and overutilisation, inter alia (Fadlallah et al., 2018). For instance, in Kenya, there is a weak regulatory framework for MHIs and coverage has been on the ability to pay, thus excluding poor households. Informal networks are associated with reduced risk of catastrophic costs, as they provide for pooling of resources among informal or mutual groups (Adisa, 2015). Informal networks and mutual organisations are conventional in many African setups, thus presenting an opportunity to leverage resources (Riddell, 2011) and possibly increase health insurance coverage.

\section{Vulnerability to shocks limits financially unprotected households' ability to invest in healthcare and other welfare services}

Vulnerability can be understood better when the types of deprivations and the kinds of adverse outcomes to which the vulnerable are predisposed are considered (Shi et al., 2008). This includes severe shocks such as loss of income, severe climatic conditions (droughts/ floods), death of a family member and loss of crops or livestock. A deficit of basic needs makes people susceptible to harm (Kohn, 2014). Severe shocks are reported to cause significant indirect effects on health and disproportionately impact the poorest and most vulnerable (L. Clarke \& Masson, 2017). Chapter 5 highlights the 
impact of income and asset losses on the households' ability to seek healthcare services. When households are economically constrained by shocks, they are faced with difficult choices and have to prioritise their resources for spending on critical needs (Monheit, 2014). Most Kenyan households have experienced shocks within a span of 5 years, with rural households experiencing higher incidence (68.7\%) of shocks, relative to urban households (53.4\%). Disparities in the incidence of shocks also exist among regions; for instance, in Kitui county, $96 \%$ of the households had experienced some form of shock, vis-a-vis only $16.8 \%$ in Kiambu county (KNBS, 2018a). Economic losses from shocks can have profound effects on the healthcare use of household members, with limited or no financial risk protection (Baulia, 2018), and can even cause households to refrain from utilising healthcare services.

WHO underscores that healthcare financing is one of the crucial components of the broader efforts to ensure social protections (WHO et al., 2010). This alludes to the critical role of social protection mechanisms in cushioning and protecting the population from financial risk provoked by healthcare payments, when confronted with unanticipated threats. Vulnerable groups like minorities, low-income populations or the uninsured are at a greater risk of poor healthcare access (Shi \& Stevens, 2005). Further, social structures such as employment, economic status, education, gender and geography have been shown to organise opportunities for healthcare (Weinstein et al., 2017).

The right to social security in Kenya is enshrined in the constitution, thus binding the state to provide appropriate social security to persons unable to provide basic services for themselves and their dependents (GOK, 2010). Eventually, the social protection policy aims to ensure that all people have the requisite financial cushion to enable them to maintain a decent living standard, including access to healthcare (GOK, 2011). Social protection mechanisms in Kenya have gradually gone through improvements with an investment of around 1.3 percent of GDP in 2016, with 0.35 per cent of the GDP going to social assistance programmes targeted at specific segments of the population, including persons with disabilities, orphans, elderly and those in foodinsecure areas (GOK, 2017a). Nonetheless, there remain significant gaps in social protection coverage due to inadequate financing, staffing capacity, poor coordination, inadequate governance and non-coverage of other vulnerable groups like unemployed and communities in areas prone to extreme weather conditions. This has left many households vulnerable to the impact of unanticipated economic shocks. Social protection is vital in building resilience to shocks and is thus an essential element to achieving equity of access in healthcare services. Increasing investments in social protection could complement investments in healthcare, thus yielding greater financial risk protection to vulnerable households in Kenya. 


\section{The application of multiple dimensions to measure equity provides more holistic and nuanced perspective on equity of access to healthcare services.}

Various measures have been proposed to quantify the different perspectives of equity in healthcare (Alonge \& Peters, 2015). Equitable healthcare financing is an objective of many healthcare systems, and $\mathrm{CHE}$ is a common indicator used to measure the degree of financial risk protection, as evidenced in Chapter 2. However, CHE does not account for those who refrain from using healthcare services and can hence create the impression of a greater degree of financial risk protection. As such, unmet need is another approach used to measure equity of access to healthcare services. The extent to which equity is achieved is a core component of healthcare system performance (Allin, Hernández-Quevedo, et al., 2010) and hence useful in monitoring progress towards UHC. Chapter 4 focuses on individuals who do not gain entry into the healthcare system due to lack of financial resources and high cost of care. There have been proposals to consider stratifying analyses according to different levels (macro, meso and micro-levels) of determinants of equity (Raine et al., 2016). This dissertation applied a multilevel approach to assess the effect of variations at the county level on unmet need for healthcare. The results showed that wide variations of cost-related unmet need to healthcare services are attributable to the differences in the socioeconomic profiles across the counties.

Evidence suggests that understanding access to healthcare across multiple deprivations can shed light on the growing disparities and identify the relative importance of the individual dimensions (Mohanty, 2012). This dissertation highlights other avoidable disparities across different groups, including regional disparities, variations in health insurance coverage and socioeconomic status.

Given the complexities associated with inequity, Chapter 3 goes beyond the general measures to analyse the inequalities in $\mathrm{CHE}$ and the drivers that sustain the inequalities over time. We note that while the overall incidence of $\mathrm{CHE}$ reduced between 2007 and 2013, the socioeconomic inequalities between the rich and poor widened, implying that the catastrophic payments worsened only for the poor. These types of nuances and deep analyses unmask disparities that overall averages would not reveal and are hence essential to inform targeted policy interventions.

\subsection{Policy implications}

The findings from this dissertation point to various policy recommendations for governments working to achieve UHC to ensure good health and wellbeing for all Sustainable Development Goal (SDG) 3. The results offer policymakers potential solutions to accelerate the achievement of equity of access, especially for the poor and most vulnerable. In this section, we discuss aspects necessary for policy implementation, and at what level the policy intervention should be aimed (national, sub-national or household). 
- $\quad$ An intuitive implication would be to increase financial investment in healthcare services and social protection, to improve financial risk protection. The investment in healthcare in Kenya is only about 6\% of the GDP, with out-of-pocket spending accounting for almost one-third of the total health expenditure. Similarly, the social protection investment as a proportion of GDP is insignificant at $1.3 \%$, considering $39 \%$ of the population live below the poverty line. The need to mobilise resources for health is continually increasing, especially at the county level. Counties have limited revenueraising authority, and are thus highly dependent on national resource transfers (Dutta et al., 2018). This calls for rethinking the modalities for increased financing at the county level, as for instance, enhancement of counties' own-resource revenue. In addition, to contribute to a growing pool of resources, some suggestions would be to broaden insurance contributions from the entire formal sector and expand coverage to the informal sector with the ability to pay. Though programmes like free maternal healthcare services and primary healthcare services for all are positive initiatives, with the limited investments and resources in health, proper segmentation of the population based on vulnerability would be beneficial to prioritise the poorest and inform long-term pro-poor policy interventions.

- $\quad$ Evidence shows that although there is a commitment by the government to UHC through devolution by investing in the health infrastructure, this is mainly uncoordinated (Oketch, 2017). Kenya introduced a decentralised healthcare system in 2013 under the 2010 constitution, partly to promote equitable access to healthcare services through improved decision space over public resource management and increased community participation. However, results show that planning and budgeting, as well as community involvement, have not improved in the early days of implementation of the decentralised system, due to lack of clarity of roles, delays in setting up the new system and inadequate capacity at the county level (Tsofa et al., 2017). This points to the need for better coordination of the decentralised structures and improved collaboration between the national and county governments, to realise the benefits for equitable healthcare delivery. This dissertation notes that the poor counties are the most affected by costrelated unmet need, relative to their wealthier counterparts. Kenya set up an 'equalisation fund' to address the unequal distribution of resources upon decentralisation. The equalisation fund employs a county development index to allocate the additional resources to counties lagging behind, using poverty, infrastructure, health and education parameters. However, the health indicators are service-specific, including maternal health, sanitation and immunisation, and do not consider equity of access indicators. Therefore, to ensure progress on UHC, the national government fundamentally needs to prioritise counties based on their needs, rather than the performance of certain services, to address the existing disparities.

- $\quad$ The analysis of this dissertation showed mixed results on the influence of health insurance in improving access to healthcare services. The health insurance measures in SSA have not been sufficient in cushioning the population, especially the poor. In Kenya, 
a major challenge for the current national health insurance has been the integration of the expanding informal sector and inclusion of the poor (Mathauer et al., 2008). Moreover, the insurance offered in Kenya has a premium, based on the ability to pay. This has contributed to the perverseness of $\mathrm{CHE}$, disparities in access and the unmet need for care evidenced in this dissertation. Recent evidence has shown the preference for non-contributory schemes by the majority of informal workers, because of affordability, equitability and ease of implementation (Okungu et al., 2018). The results also highlight the role of informal and mutual organisations in cushioning against catastrophic costs. This shows that while insurance is vital to improving access, there is a need to draw lessons and leverage informal and mutual networks/social capital that are common in the large informal population in Kenya and the region. Informal networks can lead to improvements in health insurance coverage through collective-based insurance packages and also cushion against financial risk provoked by the healthcare needs of poor individuals. It is thus recommended that the government should put in place an adequate regulatory framework and strengthen the institutional mechanisms that could integrate informal networks into the broader health financing system (Munge et al., 2019).

- $\quad$ Another policy implication relates to applying a multisectoral approach to address inequities in access. This dissertation has highlighted drivers and risks associated with access to healthcare services. Mechanisms for addressing inequity should not only focus on reducing the direct effects of the determinants of financial burden, but also address the socioeconomic inequalities and potential financial risks. Furthermore, the key drivers of inequity in Kenya go beyond the health sector to include persistent poverty, disparities in living standards and educational achievement (Ilinca et al., 2019). In addition, regional differences in availability and accessibility exist due to variations in structural financing and investments in healthcare. A multi-sectoral approach that draws synergies and efficiencies across various sectors would lead to a reduction of socioeconomic inequalities. Moreover, unless health interventions are designed to promote equity, efforts to attain UHC may lead to improvements in the national average, while inequalities worsen (WHO \& World Bank, 2017).

\subsection{Study limitations and areas of further research}

This dissertation has certain limitations, which point to areas for further research on the various emerging issues that are not covered but can provide further insights and evidence on equity of access to improve policy interventions.

- $\quad$ The main limitation of this dissertation is that it utilised cross-sectional data for analysis. Although the two studies (KHHEUS and KHIBS) used are robust and nationally representative surveys, they are limited in providing depth for analysis over time. Further, cross-sectional data analysis does not show whether catastrophic payments due to healthcare expenditure are transitory or permanent; nor does it indicate whether the shocks caused temporary or permanent losses. In light of this, future research would benefit from the use of panel data, which can provide insights on the analysis over time, 
as for instance, the long-term effect of those who refrain from using the healthcare services if the illness progresses, leading to even higher costs. In addition, it would be more suitable for the analyses to establish whether households recover from the financial burden caused by healthcare costs as well as from economic shocks.

- $\quad$ Although access to healthcare services is the result of an interaction of both demand and supply characteristics, this dissertation focussed on the demand side of the healthcare system. This is informed by the main aim of equity in access, which refers to an individual's ability to utilise healthcare services without financial hardships. Two main 'categories' of the population are assessed: those who access healthcare services and those who do not. This disregard the aspect of continuity of care, referring to people who may drop out of care for financial and other reasons, once initiated. Continuity of care assessment could provide perspectives from the supply side of the healthcare system, given that it would assess the experiences of people within the healthcare system. Further research would be more beneficial if it examines the supply side factors associated with equity of access.

- $\quad$ Lastly, the analyses are based on self-reported healthcare needs and costs. In other words, the responses are subjectively defined, which could lead to bias, given the possibility of differences in the ability of respondents across population groups to report illnesses, account for costs and interpret shocks. Further, health-seeking behaviour is complex; for example, this dissertation in Chapter 5 assumes direct interaction of income and asset shocks to provide estimates of the effects on utilisation of healthcare services. These limitations point to the need for further research on the nuances and the causal pathway in which economic shocks interplay with healthcare services. Qualitative research approaches could be valuable in analysing behavioural aspects that influence access to and utilisation of services.

\subsection{Conclusion}

This dissertation adds to the UHC discourse by applying various measures to examine different perspectives of equity in access to healthcare services. The findings are timely to policymakers, as UHC is at the top of the current global health agenda. Moreover, UHC is considered an integral component to achieving SDG 3 on ensuring healthy lives and promoting the wellbeing of all populations.

Overall, the findings reveal the existence of disparities in financial risk protection attributable to households' socioeconomic characteristics, regional variations and unanticipated risks. This demonstrates that to gauge equity of access holistically, it is vital to apply various measures, as this could unravel multiple vulnerabilities and inform targeted policy interventions. The results underscore the critical role of cost and affordability in enabling access to healthcare services. The results also highlight the important role of financial risk protection as a strategy to achieve equity of access. If financial barriers to access are removed, CHE can be prevented, and universal health coverage achieved. 
These recommendations point to the need to increase investment in healthcare and apply multi-sectoral approaches to address risks and socioeconomic inequalities that may slow-down the achievement of UHC in Kenya. The evidence also comes at a critical time when Kenya has committed to UHC in various instruments and has prioritised related programmes aimed at accelerating the achievement of UHC by 2022. While this commitment has been hailed, it is also considered to be overly ambitious, considering the evidence on current inequities and disparities highlighted in this dissertation. However, if strategic investments that promote equity in access are made, even if this goal is not achieved by 2022, the ultimate results of this commitment will be far-reaching with unprecedented beneficial effects. 


\section{References}

Aakvik, A. (2001). Bounding a Matching Estimator: The Case of a Norwegian Training Program. Oxford Bulletin of Economics and Statistics, 63(1), 115-143. https://doi.org/10.1111/1468-0084.00211

Aaltonen, K., Miettinen, J., Airio, I., Martikainen, J. E., Saastamoinen, L. K., Bell, J. S., Hartikainen, S., \& Norris, P. (2015). Cost-related barriers to use of health services and prescription medicines in Finland: A cross-sectional survey. European Journal of Public Health, 25(3), 368-372. https://doi.org/10.1093/eurpub/cku176

Abuya, T., Maina, T., \& Chuma, J. (2015). Historical account of the national health insurance formulation in Kenya: Experiences from the past decade. BMC Health Services Research, 15(1), 1-11. https://doi.org/10.1186/s12913-015-0692-8

Academy of Medical Sciences. (2017). Unmet need in healthcare Summary of a roundtable held at the Academy of Medical Sciences on 31 July 2017, held with support from the British Academy and NHS England. The Academy of Medical Sciences.

Acharya, A., Vellakkal, S., Taylor, F., Masset, E., Satija, A., Burke, M., \& Ebrahim, S. (2013). The Impact of Health Insurance Schemes for the Informal Sector in Low- and Middle-Income Countries: A Systematic Review. The World Bank Research Observer, 28(2), 236-266. https://doi.org/10.1093/wbro/lks009

Achoki, T., Miller-Petrie, M. K., Glenn, S. D., Kalra, N., Lesego, A., Gathecha, G. K., Alam, U., Kiarie, H. W., Maina, I. W., Adetifa, I. M. O., Barsosio, H. C., Degfie, T. T., Keiyoro, P. N., Kiirithio, D. N., Kinfu, Y., Kinyoki, D. K., Kisia, J. M., Krish, V. S., Lagat, A. K., ... Naghavi, M. (2019). Health disparities across the counties of Kenya and implications for policy makers, 1990-2016: A systematic analysis for the Global Burden of Disease Study 2016. The Lancet Global Health, 7(1), e81-e95. https://doi.org/10.1016/S2214109X(18)30472-8

Adebayo, E. F., Uthman, O. A., Wiysonge, C. S., Stern, E. A., Lamont, K. T., \& Ataguba, J. E. (2015). A systematic review of factors that affect uptake of community-based health insurance in low-income and middle-income countries. BMC Health Services Research, 15(1), 543. https://doi.org/10.1186/s12913-015-1179-3

Adisa, O. (2015). Investigating determinants of catastrophic health spending among poorly insured elderly households in urban Nigeria. International Journal for Equity in Health, 14, 79. https://doi.org/10.1186/s12939-015-0188-5

Akazili, J., Mclntyre, D., Kanmiki, E. W., Gyapong, J., Oduro, A., Sankoh, O., \& Ataguba, J. E. (2017). Assessing the catastrophic effects of out-of-pocket healthcare payments prior to the uptake of a nationwide health insurance scheme in Ghana. Global Health Action, 10(1), 1289735. https://doi.org/10.1080/16549716.2017.1289735

Akinkugbe, O., Chama-Chiliba, C. M., \& Tlotlego, N. (2012). Health Financing and Catastrophic Payments for Health Care: Evidence from Household-Level Survey Data in Botswana and Lesotho. African Development Review/Revue Africaine de Developpement, 24(4), 358-370. https://doi.org/10.1080/16549716.2017.1289735 
Akter, S., \& Basher, S. A. (2014). The impacts of food price and income shocks on household food security and economic well-being: Evidence from rural Bangladesh. Global Environmental Change, 25, 150-162. https://doi.org/10.1016/j.gloenvcha.2014.02.003

Alam, K., \& Mahal, A. (2014). Economic impacts of health shocks on households in low and middle income countries: A review of the literature. Globalization and Health, 10, 21. https://doi.org/10.1186/1744-8603-10-21

Alesane, A., \& Anang, B. T. (2018). Uptake of health insurance by the rural poor in Ghana: Determinants and implications for policy. The Pan African Medical Journal, 31. https://doi.org/10.11604/pamj.2018.31.124.16265

Ali, M. S., Prieto-Alhambra, D., Lopes, L. C., Ramos, D., Bispo, N., Ichihara, M. Y., Pescarini, J. M., Williamson, E., Fiaccone, R. L., Barreto, M. L., \& Smeeth, L. (2019). Propensity Score Methods in Health Technology Assessment: Principles, Extended Applications, and Recent Advances. Frontiers in Pharmacology, 10. https://doi.org/10.3389/fphar.2019.00973

Allin, S., Grignon, M., \& Le Grand, J. (2010). Subjective unmet need and utilization of health care services in Canada: What are the equity implications? Social Science \& Medicine, 70(3), 465-472. https://doi.org/10.1016/j.socscimed.2009.10.027

Allin, S., Hernández-Quevedo, C., Masseria, C., \& Leatherman, S. (2010). Measuring equity of access to health care. In P. C. Smith, E. Mossialos, \& I. Papanicolas (Eds.), Performance measurement for health system improvement (pp. 187-221). Cambridge University Press. https://doi.org/10.1017/CB09780511711800.008

Allin, S., \& Masseria, C. (2009a). Unmet need as an indicator of access to health care in Europe.

Allin, S., \& Masseria, C. (2009b). Unmet need as an indicator of health care access. Eurohealth, 15(3).

Alonge, O., \& Peters, D. (2015). Utility and limitations of measures of health inequities: A theoretical perspective. Global Health Action, 8(0). https://doi.org/10.3402/gha.v8.27591

Amponsah, S. (2016). The Incidence of Health Shocks, Formal Health Insurance, and Informal Coping Mechanisms. Perspectives on Global Development and Technology, 15(6), 665-695. https://doi.org/10.1163/15691497-12341412

Andersen, R M, McCutcheon, A., Aday, L. A., Chiu, G. Y., \& Bell, R. (1983). Exploring dimensions of access to medical care. Health Services Research, 18(1), 49-74.

Andersen, Ronald M. (1995). Revisiting the Behavioral Model and Access to Medical Care: Does it Matter? Journal of Health and Social Behavior, 36(1), 1-10. https://doi.org/10.2307/2137284

Arndt, C., McKay, A. D., Tarp, F., \& World Institute for Development Economics Research (Eds.). (2016). Growth and poverty in sub-Saharan Africa (First edition). Oxford University Press. 
Arsenault, C., Fournier, P., Philibert, A., Sissoko, K., Coulibaly, A., Tourigny, C., Traoré, M., \& Dumont, A. (2013). Emergency obstetric care in Mali: Catastrophic spending and its impoverishing effects on households. Bulletin of the World Health Organization, 91(3), 207-216. https://doi.org/10.2471/BLT.12.108969

Arsenijevic, J., Pavlova, M., \& Groot, W. (2013). Measuring the catastrophic and impoverishing effect of household health care spending in Serbia. Social Science \& Medicine, 78, 17 25. https://doi.org/10.1016/j.socscimed.2012.11.014

Ataguba, J. E.-O. (2012). Reassessing catastrophic health-care payments with a Nigerian case study. Health Economics Policy and Law, 7(3), 309-326. https://doi.org/10.1017/S1744133110000356

Atake, E.-H. (2018). Health shocks in sub-Saharan Africa: Are the poor and uninsured households more vulnerable? Health Economics Review, 8(1), 26. https://doi.org/10.1186/s13561-018-0210-x

Atake, E.-H., \& Amendah, D. D. (2018). Porous safety net: Catastrophic health expenditure and its determinants among insured households in Togo. BMC Health Services Research, 18(1). https://doi.org/10.1186/s12913-018-2974-4

Austin, P. C. (2010). Optimal caliper widths for propensity-score matching when estimating differences in means and differences in proportions in observational studies. Pharmaceutical Statistics, 10(2), 150-161. https://doi.org/10.1002/pst.433

Austin, P. C. (2011). An Introduction to Propensity Score Methods for Reducing the Effects of Confounding in Observational Studies. Multivariate Behavioral Research, 46(3), 399424. https://doi.org/10.1080/00273171.2011.568786

Awiti, C. A., Dennis, A. C. K., Mutie, C. K., Sanya, S. O., Angelique, U., Wankuru, P. C., Pape, U. J., Mistiaen, J. A., Tolchinsky, M., Chengula, L. K., Safavian, M. S., Ramana, G. N. V., Khatimba, A., Muthembwa, K. M., Rosauer, V. A. O., \& Waiharo, R. (2018). Kenya economic update: Policy options to advance the Big 4 - unleashing Kenya's private sector to drive inclusive growth and accelerate poverty reduction (No. 125056; pp. 188). The World Bank.

Awiti, J. O. (2014). Poverty and health care demand in Kenya. BMC Health Services Research, 14. https://doi.org/10.1186/s12913-014-0560-y

Ayanore, M. A., Pavlova, M., \& Groot, W. (2016). Unmet reproductive health needs among women in some West African countries: A systematic review of outcome measures and determinants. Reproductive Health, 13(1), 5. https://doi.org/10.1186/s12978-0150104-x

Babitsch, B., Gohl, D., \& von Lengerke, T. (2012). Re-revisiting Andersen's Behavioral Model of Health Services Use: A systematic review of studies from 1998-2011. GMS PsychoSocial-Medicine, 9. https://doi.org/10.3205/psm000089

Barasa, E., Maina, T., \& Ravishankar, N. (2017). Assessing the impoverishing effects, and factors associated with the incidence of catastrophic health care payments in Kenya. International Journal for Equity in Health, 16(1). https://doi.org/10.1186/s12939-0170526-x 
Barasa, E., Rogo, K., Mwaura, N., \& Chuma, J. (2018). Kenya National Hospital Insurance Fund Reforms: Implications and Lessons for Universal Health Coverage. Health Systems \& Reform, 4(4), 346-361. https://doi.org/10.1080/23288604.2018.1513267

Barasa, E. W., Nguhiu, P., \& Mclntyre, D. (2018). Measuring progress towards Sustainable Development Goal 3.8 on universal health coverage in Kenya. BMJ Global Health, 3(3), e000904. https://doi.org/10.1136/bmjgh-2018-000904

Barnes, K., Mukherji, A., Mullen, P., \& Sood, N. (2017). Financial risk protection from social health insurance. Journal of Health Economics, 55, 14-29. https://doi.org/10.1016/j.jhealeco.2017.06.002

Baulia, S. (2018). Is household shock a boon or bane to the utilisation of preventive healthcare for children? Evidence from Uganda. In Discussion Papers (No. 121; Discussion Papers). Aboa Centre for Economics. https://ideas.repec.org/p/tkk/dpaper/dp121.html

Baumbach, A., \& Gulis, G. (2014). Impact of financial crisis on selected health outcomes in Europe. European Journal of Public Health, 24(3), 399-403. https://doi.org/10.1093/eurpub/cku042

Beaulière, A., Touré, S., Alexandre, P.-K., Koné, K., Pouhé, A., Kouadio, B., Journy, N., Son, J., Ettiègne-Traoré, V., Dabis, F., Eholié, S., \& Anglaret, X. (2010). The financial burden of morbidity in HIV-infected adults on antiretroviral therapy in Côte d'Ivoire. PloS One, 5(6), e11213. https://doi.org/10.1371/journal.pone.0011213

Becker, S. O., \& Caliendo, M. (2007). Sensitivity Analysis for Average Treatment Effects. The Stata Journal: Promoting Communications on Statistics and Stata, 7(1), 71-83. https://doi.org/10.1177/1536867X0700700104

Beegle, K., Dehejia, R. H., \& Gatti, R. (2006). Child labor and agricultural shocks. Journal of Development Economics, 81(1), 80-96. https://doi.org/10.1016/j.jdeveco.2005.05.003

Bettencourt, E. M. V., Tilman, M., Narciso, V., Carvalho, M. L. da S., Henriques, P. D. de S., Bettencourt, E. M. V., Tilman, M., Narciso, V., Carvalho, M. L. da S., \& Henriques, P. D. de S. (2015). The Livestock Roles in the Wellbeing of Rural Communities of TimorLeste. Revista de Economia e Sociologia Rural, 53, 63-80. https://doi.org/10.1590/1234-56781806-94790053s01005

Blanchet, N. J., Fink, G., \& Osei-Akoto, I. (2012). The Effect of Ghana's National Health Insurance Scheme on Health Care Utilisation. Ghana Medical Journal, 46(2), 76-84.

Boerma, J. T. (2015). Tracking universal health coverage: First global monitoring report. http://apps.who.int/iris/bitstream/10665/174536/1/9789241564977_eng.pdf?ua= 1

Boing, A. C., Bertoldi, A. D., Barros, A. J. D. de, Posenato, L. G., \& Peres, K. G. (2014). Socioeconomic inequality in catastrophic health expenditure in Brazil. Revista de Saúde Pública, 48(4), 632-641. https://doi.org/10.1590/S0034-8910.2014048005111 
Bonfrer, I., \& Gustafsson-Wright, E. (2017). Health shocks, coping strategies and foregone healthcare among agricultural households in Kenya. Global Public Health, 12(11), 1369-1390. https://doi.org/10.1080/17441692.2015.1130847

Bonnefoy, J. (2010). Priorities for research on equity and health: Implications for global and national priority setting and the role.

http://wwwlive.who.int/entity/social_determinants/implementation/copyoftherevisedr esearchprioritiesforequity.pdf

Braveman, P. (2006). Health disparities and health equity: Concepts and measurement. Annu. Rev. Public Health, 27, 167-194.

Braveman, P. (2014). What are health disparities and health equity? We need to be clear. Public Health Reports, 129.

Braveman, P., \& Gruskin, S. (2003). Defining equity in health. Journal of Epidemiology and Community Health, 57(4), 254-258.

Bright, T., Felix, L., Kuper, H., \& Polack, S. (2017). A systematic review of strategies to increase access to health services among children in low and middle income countries. BMC Health Services Research, 17(1). https://doi.org/10.1186/s12913-017-2180-9

Brinda, E. M., Andrés, A. R., Andrés, R. A., \& Enemark, U. (2014). Correlates of out-of-pocket and catastrophic health expenditures in Tanzania: Results from a national household survey. BMC International Health and Human Rights, 14, 5. https://doi.org/10.1186/1472-698X-14-5

Brookhart, M. A., Schneeweiss, S., Rothman, K. J., Glynn, R. J., Avorn, J., \& Stürmer, T. (2006). Variable Selection for Propensity Score Models. American Journal of Epidemiology, 163(12), 1149-1156. https://doi.org/10.1093/aje/kwj149

Brown, A. F., Ma, G. X., Miranda, J., Eng, E., Castille, D., Brockie, T., Jones, P., Airhihenbuwa, C. O., Farhat, T., Zhu, L., \& Trinh-Shevrin, C. (2019). Structural Interventions to Reduce and Eliminate Health Disparities. American Journal of Public Health, 109(Suppl 1), S72S78. https://doi.org/10.2105/AJPH.2018.304844

Buigut, S., Ettarh, R., \& Amendah, D. D. (2015). Catastrophic health expenditure and its determinants in Kenya slum communities. International Journal for Equity in Health, 14(1). https://doi.org/10.1186/s12939-015-0168-9

Burgert-Brucker, C. R., Yourkavitch, J., Assaf, S., \& Delgado, S. (2015). Geographic Variation in Key Indicators of Maternal and Child Health Across 27 Countries in Sub-Saharan Africa.DHS Spatial Analysis Reports No. 12. Rockville, Maryland, USA: ICF International.

Burnham, K. P., \& Anderson, D. R. (2004). Multimodel Inference: Understanding AIC and BIC in Model Selection. Sociological Methods \& Research, 33(2), 261-304. https://doi.org/10.1177/0049124104268644

Carrin, G., James, C., Adelhardt, M., Doetinchem, O., Eriki, P., Hassan, M., Kirigia, J., Koemm, B., Korte, R., Krech, R., Lankers, C., van Lente, J., Maina, T., Malonza, K., Mathauer, I., Okeyo, T. M., Muchiri, S., Mumani, Z., Nganda, B., ... Zipperer, M. (2007). Health financing reform in Kenya - assessing the social health insurance proposal. 97(2), 6. 
Castillo-Riquelme, M., McIntyre, D., \& Barnes, K. (2008). Household burden of malaria in South Africa and Mozambique: Is there a catastrophic impact? Tropical Medicine \& International Health, 13(1), 108-122. https://doi.org/10.1111/j.13653156.2007.01979.x

Castro-Leal, F., Dayton, J., Demery, L., \& Mehra, K. (2000). Public spending on health care in Africa: Do the poor benefit. Inequalities in Health, 9.

Cavalieri, M. (2013). Geographical variation of unmet medical needs in Italy: A multivariate logistic regression analysis. International Journal of Health Geographics, 12, 27. https://doi.org/10.1186/1476-072X-12-27

Chakraborty, N., Wanderi, J., Oduor, C., \& Montagu, D. (2017). Assessing provision and equity in low and middle-income country health markets: A study from Kenya. Metrics for Management. https://www.psi.org/wp-content/uploads/2020/02/Assessing-Provisionand-Equity-in-LMIC-Health-Markets.pdf

Chen, J., Rizzo, J. A., \& Rodriguez, H. P. (2011). The Health Effects of Cost-Related Treatment Delays. American Journal of Medical Quality, 26(4), 261-271. https://doi.org/10.1177/1062860610390352

Cheng, T. (2015). How income influences our healthcare decisions. World Economic Forum. https://www.weforum.org/agenda/2015/08/how-income-influences-our-healthcaredecisions/

Chhabra, R., Teitelman, N., Silver, E. J., Raufman, J., \& Bauman, L. J. (2018). Vulnerability Multiplied: Health Needs Assessment of 13-18-Year-Old Female Orphan and Vulnerable Children in Kenya. World Medical \& Health Policy, 10(2), 129-145. https://doi.org/10.1002/wmh3.267

Christiaensen, L., \& Demery, L. (Eds.). (2017). Agriculture in Africa: Telling Myths from Facts. The World Bank. https://doi.org/10.1596/978-1-4648-1134-0

Chu, A., Kwon, S., \& Cowley, P. (2019). Health Financing Reforms for Moving towards Universal Health Coverage in the Western Pacific Region. Health Systems \& Reform, 5(1), 32-47. https://doi.org/10.1080/23288604.2018.1544029

Chuma, J., Gilson, L., \& Molyneux, C. (2007). Treatment-seeking behaviour, cost burdens and coping strategies among rural and urban households in Coastal Kenya: An equity analysis. Tropical Medicine \& International Health, 12(5), 673-686. https://doi.org/10.1111/j.1365-3156.2007.01825.x

Chuma, J., \& Maina, T. (2012). Catastrophic health care spending and impoverishment in Kenya. BMC Health Services Research, 12, 413. https://doi.org/10.1186/1472-6963-12-413

Chuma, J., Maina, T., \& Ataguba, J. (2012). Does the distribution of health care benefits in Kenya meet the principles of universal coverage? BMC Public Health, 12, 20. https://doi.org/10.1186/1471-2458-12-20

Chuma, J., \& Okungu, V. (2011). Viewing the Kenyan health system through an equity lens: Implications for universal coverage. International Journal for Equity in Health, 10, 22. https://doi.org/10.1186/1475-9276-10-22 
Cissé, B., Luchini, S., \& Moatti, J. P. (2007). Progressivity and horizontal equity in health care finance and delivery: What about Africa? Health Policy, 80(1), 51-68.

https://doi.org/10.1016/j.healthpol.2006.02.011

Clarke, D., \& Dercon, S. (2009). Insurance, Credit and Safety Nets for the Poor in a World of Risk. 18.

Clarke, L., \& Masson, V. L. (2017). Shocks, stresses and universal health coverage: Pathways to address resilience and health. Overseas Development Institute.

https://www.odi.org/publications/10993-shocks-stresses-and-universal-healthcoverage-pathways-address-resilience-and-health

Cleary, S., Birch, S., Chimbindi, N., Silal, S., \& McIntyre, D. (2013). Investigating the affordability of key health services in South Africa. Social Science \& Medicine (1982), 80, 37-46. https://doi.org/10.1016/j.socscimed.2012.11.035

Counts, C. J., \& Skordis-Worrall, J. (2016). Recognizing the importance of chronic disease in driving healthcare expenditure in Tanzania: Analysis of panel data from 1991 to 2010. Health Policy and Planning, 31(4), 434-443. https://doi.org/10.1093/heapol/czv081

Couttolenc, B. F. (2012). Decentralization and Governance in the Ghana Health Sector. The World Bank. https://doi.org/10.1596/978-0-8213-9589-9

CRA. (2011). Kenya country fact sheets. Commission on revenue allocation. http://siteresources.worldbank.org/INTAFRICA/Resources/2579941335471959878/Kenya_County_Fact_Sheets_Dec2011.pdf

Culyer, A. J. (2001). Equity-Some theory and its policy implications. Journal of Medical Ethics, 27(4), 275-283. https://doi.org/10.1136/jme.27.4.275

David, N. K., Kimani, D. N., \& Kinyanjui, B. (2017). Determinants and Distribution of Catastrophic Health Expenditures and Impoverishment in Kenya. Public Health Research, 7(3), 5561.

David, N., \& Wanjala, P. (2020). A Case for Increasing Public Investments in Health. 8.

Dercon, S. (2002). Income Risk, Coping Strategies, and Safety Nets. The World Bank Research Observer, 17(2), 141-166. JSTOR.

Diez-Roux, A. V. (2000). Multilevel Analysis in Public Health Research. Annual Review of Public Health, 21(1), 171-192. https://doi.org/10.1146/annurev.publhealth.21.1.171

Diwakar, V., \& Shepherd, A. (2018). A multidimensional analysis. 53.

Dorjdagva, J., Batbaatar, E., Svensson, M., Dorjsuren, B., \& Kauhanen, J. (2016). Catastrophic health expenditure and impoverishment in Mongolia. International Journal for Equity in Health, 15(1). https://doi.org/10.1186/s12939-016-0395-8

Doss, C., Oduro, A. D., \& Deere, C. D. (2015). Shocks, assets and social protection: A gendered analysis of Ecuador, Ghana, and Karnataka, India. UN Women. https://www.unwomen.org/en/digital-library/publications/2015/6/shocks-assets-andsocial-protection 
Dover, D. C., \& Belon, A. P. (2019). The health equity measurement framework: A comprehensive model to measure social inequities in health. International Journal for Equity in Health, 18(1), 36. https://doi.org/10.1186/s12939-019-0935-0

Duncan, C., Jones, K., \& Moon, G. (1993). Special Issue The Scope of Medical GeographyDo places matter? A multi-level analysis of regional variations in health-related behaviour in Britain. Social Science \& Medicine, 37(6), 725-733. https://doi.org/10.1016/02779536(93)90366-C

Dutta, A., Maina, T., Ginivan, M., \& Koseki, S. (2018). Kenya Health Financing System Assessment, 2018: Time to Pick the Best Path (p. 118). alladium, Health Policy Plus.

Dyer, S. J., Sherwood, K., McIntyre, D., \& Ataguba, J. E. (2013). Catastrophic payment for assisted reproduction techniques with conventional ovarian stimulation in the public health sector of South Africa: Frequency and coping strategies. Human Reproduction, 28(10), 2755-2764. https://doi.org/10.1093/humrep/det290

ECA. (2009). Assessing Progress in Africa towards the Millennium Development Goals. United Nations Economic Commission for Africa.

Ensor, T., \& Ronoh, J. (2005). Effective financing of maternal health services: A review of the literature. Health Policy, 75(1), 49-58.

https://doi.org/10.1016/j.healthpol.2005.02.002

Erreygers, G. (2009a). Correcting the Concentration Index: A reply to Wagstaff. Journal of Health Economics, 28(2), 521-524. https://doi.org/10.1016/j.jhealeco.2008.12.001

Erreygers, G. (2009b). Correcting the Concentration Index. Journal of Health Economics, 28(2), 504-515. https://doi.org/10.1016/j.jhealeco.2008.02.003

Essue, B. M., Kimman, M., Svenstrup, N., Lindevig Kjoege, K., Lea Laba, T., Hackett, M. L., \& Jan, S. (2015). The effectiveness of interventions to reduce the household economic burden of illness and injury: A systematic review. Bulletin of the World Health Organization, 93(2), 102-112B. https://doi.org/10.2471/BLT.14.139287

Ettarh, R. R. (2011). Spatial Analysis of Contraceptive Use and Unmet Need in Kenya.

Ezat Wan Puteh, S., \& Almualm, Y. (2017). Catastrophic Health Expenditure among Developing Countries. Health Systems and Policy Research, 04(01). https://doi.org/10.21767/2254-9137.100069

Fadlallah, R., El-Jardali, F., Hemadi, N., Morsi, R. Z., Abou Samra, C. A., Ahmad, A., Arif, K., Hishi, L., Honein-AbouHaidar, G., \& Akl, E. A. (2018). Barriers and facilitators to implementation, uptake and sustainability of community-based health insurance schemes in low- and middle-income countries: A systematic review. International Journal for Equity in Health, 17(1), 13. https://doi.org/10.1186/s12939-018-0721-4

Feinstein, L., Sabates, R., Anderson, T. M., Sorhaindo, A., \& Hammond, C. (2006). 4. What are the effects of education on health? 184.

Fella, G., Frache, S., \& Koeniger, W. (2017). Buffer-Stock Saving and Households' Response to Income Shocks. SSRN Electronic Journal. https://doi.org/10.2139/ssrn.2947137 
Fiorillo, D. (2019). Reasons for unmet needs for health care: The role of social capital and social support in some western EU countries. International Journal of Health Economics and Management. https://doi.org/10.1007/s10754-019-09271-0

Fjær, E. L., Stornes, P., Borisova, L. V., McNamara, C. L., \& Eikemo, T. A. (2017). Subjective perceptions of unmet need for health care in Europe among social groups: Findings from the European social survey (2014) special module on the social determinants of health. European Journal of Public Health, 27(suppl_1), 82-89. https://doi.org/10.1093/eurpub/ckw219

Fortney, J. C., Burgess, J. F., Bosworth, H. B., Booth, B. M., \& Kaboli, P. J. (2011). A Reconceptualization of Access for 21st Century Healthcare. Journal of General Internal Medicine, 26(S2), 639-647. https://doi.org/10.1007/s11606-011-1806-6

Friedman, E. (2015, October 6). SDG SERIES: National Health Equity Strategies to Implement the Global Promise of SDGs. Health and Human Rights Journal. https://www.hhrjournal.org/2015/10/sdg-series-national-health-equity-strategies-toimplement-the-global-promise-of-sdgs/

Friedrich-Ebert-Stiftung. (2012). Regional disparities and marginalisation in Kenya (p. 104). Friedrich-Ebert-Stiftung.

Fullan, M. (2000). The return of large-scale reform. Journal of Educational Change, 1(1), 5-27.

Ganesh, L. (2015). Impact of indirect cost on access to healthcare utilization. International Journal of Medical Science and Public Health, 4(9), 1255. https://doi.org/10.5455/ijmsph.2015.28012015258

Garrido, M. M., Kelley, A. S., Paris, J., Roza, K., Meier, D. E., Morrison, R. S., \& Aldridge, M. D. (2014). Methods for Constructing and Assessing Propensity Scores. Health Services Research, 49(5), 1701-1720. https://doi.org/10.1111/1475-6773.12182

Gelormino, E., Bambra, C., Spadea, T., Bellini, S., \& Costa, G. (2011). The Effects of Health Care Reforms on Health Inequalities: A Review and Analysis of the European Evidence Base. International Journal of Health Services, 41(2), 209-230. https://doi.org/10.2190/HS.41.2.b

Ghafoori, M. H., Azar, F. E., Arab, M., Mahmoodi, M., Zadeh, N. Y., \& Rezapour, A. (2014). The Distribution of Health Expenditures in Tehran's Districts. 13.

Glennerster, H., Bradshaw, J., Lister, R., \& Lundberg, O. (2009). Reducing the Risks to Health: The role of social protection. 49.

Goddard, M., \& Smith, P. (2001). Equity of access to health care services: Theory and evidence from the UK. Social Science \& Medicine, 53(9), 1149-1162.

GOK. (2007). Kenya Vision 2030. Government of Kenya.

GOK. (2010). The constitution of Kenya, 2010. Government of Kenya.

GOK. (2011). Kenya National Social protection Policy. Government of Kenya.

GOK. (2017a). Kenya Social Protection Sector Review 2017 report. Ministry of Labour and Social Protection. https://www.socialprotection.go.ke/wp-content/uploads/2019/10/KENYASOCIAL-PROTECTION-SECTOR-REVIEW-FULL-DOC.pdf 
GOK. (2017b). “The Big Four” - Immediate priorities and actions.

Goryakin, Y., \& Suhrcke, M. (2014). The prevalence and determinants of catastrophic health expenditures attributable to non-communicable diseases in low- and middle-income countries: A methodological commentary. International Journal for Equity in Health, 13. https://doi.org/10.1186/s12939-014-0107-1

Gottret, P. E., \& Schieber, G. (2006). Health Financing Revisited: A Practitioner's Guide. The World Bank. https://doi.org/10.1596/978-0-8213-6585-4

Gray, B., \& Gash, M. (2016). Designing Financial Services to Respond to Household Shocks: A Case Study of RCPB's Health Savings and Loan Product (p. 10). Consultative Group to Assist the Poor(CGAP). https://www.cgap.org/research/publication/designing-financialservices-respond-household-shocks

Gribble, J., \& Haffey, J. (2008). Reproductive health in sub-Saharan Africa. Population Reference Bureau, 8.

Gulliford, M., Figueroa-Munoz, J., Morgan, M., Hughes, D., Gibson, B., Beech, R., \& Hudson, M. (2002). What does "access to health care" mean? Journal of Health Services Research \& Policy, 7(3), 186-188.

Gulliford, M., \& Morgan, M. (Eds.). (2003). Access to health care. Routledge.

Guure, C., Maya, E. T., Dery, S., da-Costa Vrom, B., Alotaibi, R. M., Rezk, H. R., \& Yawson, A. (2019). Factors influencing unmet need for family planning among Ghanaian married/union women: A multinomial mixed effects logistic regression modelling approach. Archives of Public Health, 77(1), 11. https://doi.org/10.1186/s13690-0190340-6

Haghparast-Bidgoli, H., Pulkki-Brännström, A.-M., Lafort, Y., Beksinska, M., Rambally, L., Roy, A., Reza-Paul, S., Ombidi, W., Gichangi, P., \& Skordis-Worrall, J. (2015). Inequity in costs of seeking sexual and reproductive health services in India and Kenya. International Journal for Equity in Health, 14(1). https://doi.org/10.1186/s12939-015-0216-5

Harris, B., Goudge, J., Ataguba, J. E., McIntyre, D., Nxumalo, N., Jikwana, S., \& Chersich, M. (2011). Inequities in access to health care in South Africa. Journal of Public Health Policy, 32(1), S102-S123.

Heckley, G., Gerdtham, U.-G., \& Kjellsson, G. (2016). A general method for decomposing the causes of socioeconomic inequality in health. Journal of Health Economics, 48, 89106. https://doi.org/10.1016/j.jhealeco.2016.03.006

Hodgson, H. (2010). Theories of Distributive Justice: Frameworks for Equity.

Hoogeveen, J., Tesliuc, E., \& Vakis, R. (2005). A Guide to the Analysis of Risk, Vulnerability and Vulnerable Groups. 41.

Hosseinpoor, A. R., Bergen, N., \& Schlotheuber, A. (2015). Promoting health equity: WHO health inequality monitoring at global and national levels. Global Health Action, 8(0). https://doi.org/10.3402/gha.v8.29034 
Huang, J., Birkenmaier, J., \& Kim, Y. (2014). Job Loss and Unmet Health Care Needs in the Economic Recession: Different Associations by Family Income. American Journal of Public Health, 104(11), e178-e183. https://doi.org/10.2105/AJPH.2014.301998

Hwang, J. (2018). Understanding reasons for unmet health care needs in Korea: What are health policy implications? BMC Health Services Research, 18(1), 557. https://doi.org/10.1186/s12913-018-3369-2

Ichoku, H. E., Fonta, W., \& Onwujekwe, O. (2009). Incidence and intensity of catastrophic healthcare financing and impoverishment due to out-of-pocket payments in southeast Nigeria. Journal of Insurance and Risk Management, 4(4), 47-59.

Ichoku, H. Eme, \& Fonta, W. M. (2009). Catastrophic Healthcare Financing and Poverty: Empirical Evidence from Nigeria. Journal of Social and Economic Development, 11(2), 1-16.

IHME. (2014). Health Service Provision in Kenya: Assessing Facility Capacity, Costs of Care, and Patient Perspectives. Institute for Health Metrics and Evaluation(IHME), Seattle, WA, 78.

Ilinca, S., Di Giorgio, L., Salari, P., \& Chuma, J. (2019). Socio-economic inequality and inequity in use of health care services in Kenya: Evidence from the fourth Kenya household health expenditure and utilization survey. International Journal for Equity in Health, 18(1), 196. https://doi.org/10.1186/s12939-019-1106-z

ILO. (2008). Social health protection: An ILO strategy towards universal access to health care. International Labour Office.

ILO. (2012). Social Protection Floors Recommendation, 2012 (No. 202). 12.

Ilunga-llunga, F., Levêque, A., Laokri, S., \& Dramaix, M. (2015). Incidence of catastrophic health expenditures for households: An example of medical attention for the treatment of severe childhood malaria in Kinshasa reference hospitals, Democratic Republic of Congo. Journal of Infection and Public Health, 8(2), 136-144. https://doi.org/10.1016/j.jiph.2014.08.008

Isaac, V., McLachlan, C. S., Baune, B. T., Huang, C.-T., \& Wu, C.-Y. (2015). Poor Self-Rated Health Influences Hospital Service Use in Hospitalized Inpatients With Chronic Conditions in Taiwan. Medicine, 94(36), e1477. https://doi.org/10.1097/MD.0000000000001477

Jacobs, B., Ir, P., Bigdeli, M., Annear, P. L., \& Van Damme, W. (2012). Addressing access barriers to health services: An analytical framework for selecting appropriate interventions in low-income Asian countries. Health Policy and Planning, 27(4), 288-300. https://doi.org/10.1093/heapol/czr038

Jaspers, L., Colpani, V., Chaker, L., van der Lee, S. J., Muka, T., Imo, D., Mendis, S., Chowdhury, R., Bramer, W. M., Falla, A., Pazoki, R., \& Franco, O. H. (2015). The global impact of non-communicable diseases on households and impoverishment: A systematic review. European Journal of Epidemiology, 30(3), 163-188. https://doi.org/10.1007/s10654014-9983-3

Jivetti, B., Alcántara, A. N., Miller, J. A., \& Rhatigan, R. (2018). FINANCING HEALTH CARE IN KENYA: ARE KENYANS WILLING TO PAY HIGHER TAXES FOR BETTER HEALTH CARE? 30. 
Kabia, E., Mbau, R., Oyando, R., Oduor, C., Bigogo, G., Khagayi, S., \& Barasa, E. (2019). “We are called the et cetera": Experiences of the poor with health financing reforms that target them in Kenya. International Journal for Equity in Health, 18(1), 1-14. https://doi.org/10.1186/s12939-019-1006-2

Kakwani, N., Wagstaff, A., \& Van Doorslaer, E. (1997). Socioeconomic Inequalities in Health: Measurement, Computation and Statistical Inference. 77, 87-103.

Kankeu, H. T., Saksena, P., Xu, K., \& Evans, D. B. (2013). The financial burden from noncommunicable diseases in low- and middle-income countries: A literature review. Health Research Policy and Systems, 11, 31. https://doi.org/10.1186/1478-4505-1131

Kazungu, J. S., \& Barasa, E. W. (2017). Examining levels, distribution and correlates of health insurance coverage in Kenya. Tropical Medicine \& International Health, 22(9), 11751185. https://doi.org/10.1111/tmi.12912

Kent Ranson, M., Sinha, T., Chatterjee, M., Acharya, A., Bhavsar, A., Morris, S. S., \& Mills, A. J. (2006). Making health insurance work for the poor: Learning from the Self-Employed Women's Association's (SEWA) community-based health insurance scheme in India. Social Science \& Medicine, 62(3), 707-720. https://doi.org/10.1016/j.socscimed.2005.06.037

Khan, K. S., Kunz, R., Kleijnen, J., \& Antes, G. (2003). Five steps to conducting a systematic review. Journal of the Royal Society of Medicine, 96(3), 118-121.

Kien, V. D., Van Minh, H., Giang, K. B., Dao, A., Tuan, L. T., \& Ng, N. (2016). Socioeconomic inequalities in catastrophic health expenditure and impoverishment associated with non-communicable diseases in urban Hanoi, Vietnam. International Journal for Equity in Health, 15(1). https://doi.org/10.1186/s12939-016-0460-3

Kilbourne, A. M., Switzer, G., Hyman, K., Crowley-Matoka, M., \& Fine, M. J. (2006). Advancing Health Disparities Research Within the Health Care System: A Conceptual Framework. American Journal of Public Health, 96(12), 2113-2121. https://doi.org/10.2105/AJPH.2005.077628

Kim, J., Kim, T. H., Park, E.-C., \& Cho, W. H. (2013). Factors influencing unmet need for health care services in Korea. Asia-Pacific Journal of Public Health, 27(2), NP2555-2569. https://doi.org/10.1177/1010539513490789

Kimani, D., \& Maina, T. (2016). Catastrophic Health Expenditures And Impoverishment In Kenya. European Scientific Journal, 12(15). https://doi.org/10.19044/esj.2016.v12n15p434

Kimball, M., Phily, C., Folsom, A., Lagomarsino, G., \& Holtz, J. (2013). LEVERAGING HEALTH MICROINSURANCE TO PROMOTE UNIVERSAL HEALTH COVERAGE. 23, 94.

Kimenyi, M. S., Mwega, F. M., Ndung'u, N. S., \& UNU-WIDER. (2015). The African Lions: Kenya country case study (134th ed., Vol. 2015). UNU-WIDER. https://doi.org/10.35188/UNU-WIDER/2015/023-2 
Kiringai, J., Puerta, S., \& Laura, M. (2016). Kenya economic update: Kazi ni kazi-Informal should not be normal (Working Paper No. 104289). World Bank.

http://documents.worldbank.org/curated/en/888091467988907587/Kenya-

Economic-update-kazi-ni-kazi-informal-should-not-be-normal

Kjellsson, G., \& Gerdtham, U.-G. (2014). Measuring Health Inequalities Using the Concentration Index Approach. In Encyclopedia of Health Economics (pp. 240-246). Elsevier. https://doi.org/10.1016/B978-0-12-375678-7.00206-6

Kjellsson, Gustav, \& Gerdtham, U.-G. (2013). On correcting the concentration index for binary variables. Journal of Health Economics, 32(3), 659-670.

https://doi.org/10.1016/j.jhealeco.2012.10.012

KNBS. (2018a). Basic report: Based on 2015/16 Kenya integrated household budget survey (KIHBS). Kenya National Bureau of Statistics.

KNBS. (2018b). Basic report on well-being in Kenya: Based on the 2015/16 Kenya Integrated Household Budget Survey (KIHBS). Kenya National Bureau of Statistics.

KNBS, \& SID. (2013). Exploring Kenya's Inequality: Pulling Apart or Pooling Together?

Kohn, N. A. (2014). Vulnerability theory and the role of government. Yale Journal of Law \& Feminism, 26(1). http://papers.ssrn.com/sol3/papers.cfm?abstract_id=2562737

Kristjanson, P., Mango, N., Krishna, A., Radeny, M., \& Johnson, N. (2010). Understanding poverty dynamics in Kenya. Journal of International Development, 22(7), 978-996. https://doi.org/10.1002/jid.1598

Kunna, R., San Sebastian, M., \& Stewart Williams, J. (2017). Measurement and decomposition of socioeconomic inequality in single and multimorbidity in older adults in China and Ghana: Results from the WHO study on global AGEing and adult health (SAGE). International Journal for Equity in Health, 16. https://doi.org/10.1186/s12939-0170578-y

Kutzin, J. (2013). Health financing for universal coverage and health system performance: Concepts and implications for policy. Bulletin of the World Health Organization, 91(8), 602-611. https://doi.org/10.2471/BLT.12.113985

Kutzin, J. \& others. (2008). Health financing policy: A guide for decision-makers. Health Financing Policy Paper. Copenhagen, WHO Regional Office for Europe, 24.

http://qmplus.qmul.ac.uk/pluginfile.php/158457/mod_book/chapter/3328/Kutzin\%2 02008\%20health\%20financing\%20guidance.pdf

Kutzin, J., Yip, W., \& Cashin, C. (2016). Alternative Financing Strategies for Universal Health Coverage. In World Scientific Handbook of Global Health Economics and Public Policy (Vol. 1-3, pp. 267-309). WORLD SCIENTIFIC. https://doi.org/10.1142/9789813140493_0005

Kwakye, E. (2017). Household Health Care Expenditure and Catastrophic Payments: Evidence From The Ghana Living Standards Survey V. 22.

Kwesiga, B., Zikusooka, C. M., \& Ataguba, J. E. (2015). Assessing catastrophic and impoverishing effects of health care payments in Uganda. BMC Health Services Research, 15(1). https://doi.org/10.1186/s12913-015-0682-x 
Laokri, S., Dramaix-Wilmet, M., Kassa, F., Anagonou, S., \& Dujardin, B. (2014). Assessing the economic burden of illness for tuberculosis patients in Benin: Determinants and consequences of catastrophic health expenditures and inequities. Tropical Medicine \& International Health: $T M \& I H, 19(10), 1249-1258$.

https://doi.org/10.1111/tmi.12365

Lee, S. E., Yeon, M., Kim, C.-W., \& Yoon, T.-H. (2016). The Association Among Individual and Contextual Factors and Unmet Healthcare Needs in South Korea: A Multilevel Study Using National Data. Journal of Preventive Medicine and Public Health, 49(5), 308322. https://doi.org/10.3961/jpmph.16.035

Leive, A. (2008). Coping with out-of-pocket health payments: Empirical evidence from 15 African countries. Bulletin of the World Health Organization, 86(11), 849-856.

https://doi.org/10.2471/BLT.07.049403

Levac, D., Colquhoun, H., \& O’Brien, K. K. (2010). Scoping studies: Advancing the methodology. Implementation Science, 5(1). https://doi.org/10.1186/1748-5908-5-69

Li, X., Chen, M., Wang, Z., \& Si, L. (2018). Forgone care among middle aged and elderly with chronic diseases in China: Evidence from the China Health and Retirement Longitudinal Study Baseline Survey. BMJ Open, 8(3), e019901. https://doi.org/10.1136/bmjopen2017-019901

Li, Y., Wu, Q., Xu, L., Legge, D., Hao, Y., Gao, L., Ning, N., \& Wan, G. (2012). Factors affecting catastrophic health expenditure and impoverishment from medical expenses in China: Policy implications of universal health insurance. Bulletin of the World Health Organization, 90(9), 664-671. https://doi.org/10.2471/BLT.12.102178

Limwattananon, S., Tangcharoensathien, V., \& Prakongsai, P. (2007). Catastrophic and poverty impacts of health payments: Results from national household surveys in Thailand. Bulletin of the World Health Organization, 85(8), 600-606.

Lindelow, M., \& Wagstaff, A. (2005). Can Insurance Increase Financial Risk? The Curious Case Of Health Insurance In China. The World Bank. https://doi.org/10.1596/1813-94503741

Lunt, M. (2014). Selecting an Appropriate Caliper Can Be Essential for Achieving Good Balance With Propensity Score Matching. American Journal of Epidemiology, 179(2), 226-235. https://doi.org/10.1093/aje/kwt212

Luoma, M., Doherty, J., Muchiri, S., Barasa, T., \& Hofler, K. (2010). Kenya Health System Assessment 2010. Health Systems 20/20 project, Abt Associates Inc.

Lusardi, A., Schneider, D. J., \& Tufano, P. (2010). The economic crisis and medical care usage (Working Paper No. 15843). National Bureau of Economic Research. https://doi.org/10.3386/w15843

Machiyama, K., \& Cleland, J. (2013). Insights into unmet need in Kenya (pp. 1-29) [STEP UP Research Report]. London School of Hygiene \& Tropical Medicine. https://assets.publishing.service.gov.uk/media/57a08a3640f0b649740004aa/STEPUP-unmet-FP-need-Kenya.pdf 
MacLachlan, M., Amin, M., Mannan, H., El Tayeb, S., Bedri, N., Swartz, L., Munthali, A., Van Rooy, G., \& McVeigh, J. (2012). Inclusion and Human Rights in Health Policies: Comparative and Benchmarking Analysis of 51 Policies from Malawi, Sudan, South Africa and Namibia. PLoS ONE, 7(5), e35864. https://doi.org/10.1371/journal.pone.0035864

Maina, T., \& Ongut, E. (2014). Health Policy and Abolition of User Fees at Public Primary Healthcare Facilities. 8.

Masiye, F., Kaonga, O., \& Kirigia, J. M. (2016). Does User Fee Removal Policy Provide Financial Protection from Catastrophic Health Care Payments? Evidence from Zambia. PloS One, 11(1), e0146508. https://doi.org/10.1371/journal.pone.0146508

Mathauer, I., Schmidt, J.-O., \& Wenyaa, M. (2008). Extending social health insurance to the informal sector in Kenya. An assessment of factors affecting demand. The International Journal of Health Planning and Management, 23(1), 51-68.

https://doi.org/10.1002/hpm.914

Mazumdar, S., Mazumdar, P. G., Kanjilal, B., \& Singh, P. K. (2014). Multiple Shocks, Coping and Welfare Consequences: Natural Disasters and Health Shocks in the Indian Sundarbans. PLoS ONE, 9(8), e105427. https://doi.org/10.1371/journal.pone.0105427

Mbau, R., Kabia, E., Honda, A., Hanson, K., \& Barasa, E. (2020). Examining purchasing reforms towards universal health coverage by the National Hospital Insurance Fund in Kenya. International Journal for Equity in Health, 19. https://doi.org/10.1186/s12939-0191116-x

McCollum, R., Taegtmeyer, M., Otiso, L., Mireku, M., Muturi, N., Martineau, T., \& Theobald, S. (2019). Healthcare equity analysis: Applying the Tanahashi model of health service coverage to community health systems following devolution in Kenya. International Journal for Equity in Health, 18(1), 65. https://doi.org/10.1186/s12939-019-0967-5

Mchenga, M., Chirwa, G. C., \& Chiwaula, L. S. (2017). Impoverishing effects of catastrophic health expenditures in Malawi. International Journal for Equity in Health, 16(1), 25. https://doi.org/10.1186/s12939-017-0515-0

McMurdo, M. E. T. (2000). A healthy old age: Realistic or futile goal? BMJ : British Medical Journal, 321(7269), 1149-1151.

McPeak, J. (2004). Contrasting income shocks with asset shocks: Livestock sales in northern Kenya. Oxford Economic Papers, 56(2), 263-284. https://doi.org/10.1093/oep/gpf040

Mendoza, R. U. (2009). Aggregate Shocks, Poor Households and Children: Transmission Channels and Policy Responses. SSRN Electronic Journal. https://doi.org/10.2139/ssrn.1366747

Mills, A., Ataguba, J. E., Akazili, J., Borghi, J., Garshong, B., Makawia, S., Mtei, G., Harris, B., Macha, J., Meheus, F., \& McIntyre, D. (2012). Equity in financing and use of health care in Ghana, South Africa, and Tanzania: Implications for paths to universal coverage. Lancet (London, England), 380(9837), 126-133. https://doi.org/10.1016/S01406736(12)60357-2

MOH. (2010). Kenya National Health Accounts 2009/10. Ministry of Health, Kenya. 
MOH. (2013a). Kenya Health Sector Strategic and Investment Plan KENYA HEALTH SECTOR STRATEGIC AND INVESTMENT PLAN (KHSSPI) JULY 2013-JUNE 2017. Ministry of Health, Nairobi Kenya.

MOH. (2013b). Transforming Health: Accelerating attainment of Universal Health Coverage: Kenya Health Sector Strategic and Investment Plan (KHSSPI) 2014-2018. Ministry of Health, Nairobi Kenya.

MOH. (2014a). 2013 Kenya Household Health Expenditure and Utilisation Survey. Ministry of Health, Kenya.

MOH. (2014b). Health Sector Human Resources Strategy 2014 - 2018. Ministry of Health, Nairobi Kenya.

MOH. (2014c). Kenya Service Availability and Readiness Assessment Mapping (SARAM). Ministry of Health, Nairobi Kenya.

MOH. (2014d). Kenya Health Policy 2014-2030. Ministry of Health. http://www.health.go.ke

Mohanty, S. K. (2012). Multiple Deprivations and Maternal Care in India. International Perspectives on Sexual and Reproductive Health, 38(01), 006-014. https://doi.org/10.1363/3800612

Moher, D., Shamseer, L., Clarke, M., Ghersi, D., Liberati, A., Petticrew, M., Shekelle, P., Stewart, L. A., \& PRISMA-P Group. (2015). Preferred reporting items for systematic review and meta-analysis protocols (PRISMA-P) 2015 statement. Systematic Reviews, 4(1), 1. https://doi.org/10.1186/2046-4053-4-1

Mondal, S., Kanjilal, B., Peters, D. H., \& Lucas, H. (2010). Catastrophic out-of-pocket payment for health care and its impact on households: Experience from West Bengal, India. Future Health Systems, Innovations for Equity. http://www.chronicpoverty.org/uploads/publication_files/mondal_et_al_health.pdf

Monheit, A. (2014). How Does Family Health Care Use Respond to Economic Shocks? Realized and Anticipated Effects (p. 43). National Bureau of Economic Research. http://www.nber.org/papers/w20348

Mugo, P., Onsomu, E., Munga, B., Mbithi, J., \& Owino, E. (2018). An Assessment of Healthcare Delivery in Kenya under the Devolved System. KIPPRA, Special Paper No. 19/2018, 146.

Muhammed, K. A., Umeh, K. N., Nasir, S. M., \& Suleiman, I. H. (2013). Understanding the barriers to the utilization of primary health care in a low-income setting: Implications for health policy and planning. Journal of Public Health in Africa, e13-e13. https://doi.org/10.4081/jphia.2013.e13

Muiya, B. M., \& Kamau, A. (2013). Universal Health Care in Kenya: Opportunities and challenges for the informal sector workers. http://etd-library.ku.ac.ke/handle/123456789/12224

Mulaki, A., \& Muchiri, S. (2019). Kenya Health System Assessment. Palladium, Health Policy Plus. Washington, DC, 123.

Munge, K., \& Briggs, A. H. (2014). The progressivity of health-care financing in Kenya. Health Policy and Planning, 29(7), 912-920. https://doi.org/10.1093/heapol/czt073 
Munge, K., Mulupi, S., Barasa, E., \& Chuma, J. (2019). A critical analysis of purchasing arrangements in Kenya: The case of micro health insurance. BMC Health Services Research, 19(1), 45. https://doi.org/10.1186/s12913-018-3863-6

Munge, K., Mulupi, S., \& Chuma, J. (2015). A Critical analysis of the purchasing arrangements in Kenya: The case of the National Hospital Insurance Fund, Private and Communitybased health insurance.

http://resyst.lshtm.ac.uk/sites/resyst.Ishtm.ac.uk/files/docs/reseources/Working\%20 Paper\%207\%20Kenya\%20purchasing\%20report.pdf

Murray, C. J. L., Xu, K., Klavus, J., \& Kawabata, K. (2003). Assessing the Distribution of Household Financial Contributions to the Health System: Concepts and Empirical Application. 19.

Musango, L., Elovainio, R., Nabyonga, J., \& Toure, B. (2013). The state of health financing in the African Region. 16, 6.

Mussa, R. (2016). Exit from catastrophic health payments: A method and an application to Malawi. International Journal of Health Economics and Management, 16(2), 163-174. https://doi.org/10.1007/s10754-015-9184-y

Mutangadura, G., Gauci, A., Armah, B., Woldermariam, E., Ayalew, D., \& Egu, B. (2007). Health inequities in selected African countries: Review of evidence and policy implications. Economic Commission for Africa. http://www.afdb.org/fileadmin/uploads/afdb/Documents/Knowledge/Conference_20 07_anglais_21-part-V-1.pdf

National Research Council. (2001). Under the weather: Climate, ecosystems, and infectious disease. National Academy Press. http://site.ebrary.com/id/10038772

NCAPD, MOH, \& CBS. (2005). Kenya Service Provision Assessment Survey 2004. National Coordinating Agency for Population and Development, Ministry of Health, Central Bureau of Statistics, and ORC Macro.

NCAPD, MOH, \& CBS. (2014). Kenya Service Provision Assessment Survey 2004. National Coordinating Agency for Population and Development, Ministry of Health, Central Bureau of Statistics, and ORC Macro.

NCAPD, MOMS, MOPHS, KNBS, \& ICF Macro. (2011). Kenya Service Provision Assessment Survey 2010. National Coordinating Agency for Population and Development, Ministry of Medical Services, Ministry of Public Health and Sanitation, Kenya National Bureau of Statistics, and ICF Macro.

Ndavi, P. M., Ogola, S., Kizito, P. M., \& Johnson, K. (2009). Decentralizing Kenya's Health Management System: An Evaluation (Kenya Working Papers No. 1. No. 1.). Macro International Inc. https://dhsprogram.com/pubs/pdf/WPK1/WPK1.pdf

Ngugi, A. K., Agoi, F., Mahoney, M. R., Lakhani, A., Mang'ong'o, D., Nderitu, E., Armstrong, R., \& Macfarlane, S. (2017). Utilization of health services in a resource-limited rural area in Kenya: Prevalence and associated household-level factors. PLOS ONE, 12(2). https://doi.org/10.1371/journal.pone.0172728 
Niëns, L. M., Cameron, A., Van de Poel, E., Ewen, M., Brouwer, W. B. F., \& Laing, R. (2010). Quantifying the Impoverishing Effects of Purchasing Medicines: A Cross-Country Comparison of the Affordability of Medicines in the Developing World. PLoS Medicine, 7(8), e1000333. https://doi.org/10.1371/journal.pmed.1000333

Nikoloski, Z., Christiaensen, L., \& Hill, R. (2017). Coping with Shocks: The Realities of African Life. In Agriculture in Africa: Telling Myths from Facts (Vol. 1-0, pp. 123-134). The World Bank. https://doi.org/10.1596/978-1-4648-1134-0_ch14

Nyanjom, O. (2006). Inequality in Kenya's Health Sector. In Society for International Development, 156.

Obare, F., Abuya, T., Matanda, D., \& Bellows, B. (2018). Assessing the community-level impact of a decade of user fee policy shifts on health facility deliveries in Kenya, 2003-2014. International Journal for Equity in Health, 17(1), 65. https://doi.org/10.1186/s12939018-0774-4

Odaga, J. (2004). Health inequity in Uganda: The role of financial and non-financial barriers. https://tspace.library.utoronto.ca/handle/1807/6059

ODI. (2016). Leaving no one behind in the health sector. An SDG stocktake in Kenya and Nepal. Overseas Development Institute.

O'Donnell, O. (2007). Access to health care in developing countries: Breaking down demand side barriers. Cadernos de Saúde Pública, 23(12), 2820-2834.

O’Donnell, O., van Doorslaer, E., Wagstaff, A., \& Lindelow, M. (2007). Analyzing Health Equity Using Household Survey Data: A Guide to Techniques and their Implementation. The World Bank. http://elibrary.worldbank.org/doi/book/10.1596/978-0-8213-6933-3

OECD. (2011). Health at a Glance 2011. OECD Publishing. https://doi.org/10.1787/health_glance-2011-en

OECD. (2017). Unmet needs for health care due to cost. https://doi.org/10.1787/health_glance2017-25-en

OECD, \& WHO (Eds.). (2003). Poverty and health. OECD ; World Health Organization.

Oketch, T. C. (2017). Devolution of Public Health care Services in Kenya and its Implication on Universal Health Coverage. IOSR Journal of Pharmacy (IOSRPHR), 07(05), 09-23. https://doi.org/10.9790/3013-0705010923

Okoronkwo, I. L., Ekpemiro, J. N., Okwor, E. U., Okpala, P. U., \& Adeyemo, F. O. (2015). Economic burden and catastrophic cost among people living with type2 diabetes mellitus attending a tertiary health institution in south-east zone, Nigeria. BMC Research Notes, 8, 527. https://doi.org/10.1186/s13104-015-1489-x

Okungu, V., Chuma, J., Mulupi, S., \& McIntyre, D. (2018). Extending coverage to informal sector populations in Kenya: Design preferences and implications for financing policy. BMC Health Services Research, 18(1), 13. https://doi.org/10.1186/s12913-017-2805-z

Omonona, B. T., Obisesan, A. A., \& Aromolaran, O. A. (2015). Health-care access and utilization among rural households in Nigeria. Journal of Development and Agricultural Economics, 7(5), 195-203. https://doi.org/10.5897/JDAE2014.0620 
Onwujekwe, O., Dike, N., Chukwuka, C., Uzochukwu, B., Onyedum, C., Onoka, C., \& Ichoku, H. (2009). Examining catastrophic costs and benefit incidence of subsidized antiretroviral treatment (ART) programme in south-east Nigeria. Health Policy (Amsterdam, Netherlands), 90(2-3), 223-229. https://doi.org/10.1016/j.healthpol.2008.10.006

Onwujekwe, O., Dike, N., Uzochukwu, B., \& Ezeoke, O. (2010). Informal payments for healthcare: Differences in expenditures from consumers and providers perspectives for treatment of malaria in Nigeria. Health Policy (Amsterdam, Netherlands), 96(1), 72-79. https://doi.org/10.1016/j.healthpol.2009.12.014

Onwujekwe, O. E., Ibe, O., Torpey, K., Dada, S., Uzochukwu, B., \& Sanwo, O. (2016). Examining geographic and socio-economic differences in outpatient and inpatient consumer expenditures for treating HIV/AIDS in Nigeria. Journal of the International Aids Society, 19, 20588. https://doi.org/10.7448/IAS.19.1.20588

Onwujekwe, O., Hanson, K., \& Uzochukwu, B. (2012). Examining Inequities in Incidence of Catastrophic Health Expenditures on Different Healthcare Services and Health Facilities in Nigeria. PLOS ONE, 7(7), e40811. https://doi.org/10.1371/journal.pone.0040811

Onwujekwe, O., Hanson, K., Uzochukwu, B., Ichoku, H., Ike, E., \& Onwughalu, B. (2010). Are malaria treatment expenditures catastrophic to different socio-economic and geographic groups and how do they cope with payment? A study in southeast Nigeria. Tropical Medicine \& International Health: TM \& IH, 15(1), 18-25. https://doi.org/10.1111/j.1365-3156.2009.02418.x

Opwora, A., Waweru, E., Toda, M., Noor, A., Edwards, T., Fegan, G., Molyneux, S., \& Goodman, C. (2015). Implementation of patient charges at primary care facilities in Kenya: Implications of low adherence to user fee policy for users and facility revenue. Health Policy and Planning, 30(4), 508-517. https://doi.org/10.1093/heapol/czu026

Otieno, P. O., \& Asiki, G. (2020). Making Universal Health Coverage Effective in Low- and MiddleIncome Countries: A Blueprint for Health Sector Reforms. In Healthcare AccessRegional Overviews [Working Title]. IntechOpen. https://doi.org/10.5772/intechopen.91414

Otieno, P. O., Wambiya, E. O. A., Mohamed, S. F., Donfouet, H. P. P., \& Mutua, M. K. (2019). Prevalence and factors associated with health insurance coverage in resource-poor urban settings in Nairobi, Kenya: A cross-sectional study. BMJ Open, 9(12), e031543. https://doi.org/10.1136/bmjopen-2019-031543

Park, S., \& Lake, E. T. (2005). Multilevel Modeling of a Clustered Continuous Outcome. Nursing Research, 54(6), 406-413.

Parry, J.-E., Echeverria, D., Dekens, J., \& Maitima, J. (2012). Climate Risks, Vulnerability and Governance in Kenya: A review. 83.

Peters, D. H., Garg, A., Bloom, G., Walker, D. G., Brieger, W. R., \& Hafizur Rahman, M. (2008). Poverty and Access to Health Care in Developing Countries. Annals of the New York Academy of Sciences, 1136(1), 161-171. https://doi.org/10.1196/annals.1425.011 
Peters, M. D. J., Godfrey, C. M., Khalil, H., Mclnerney, P., Parker, D., \& Soares, C. B. (2015).

Guidance for conducting systematic scoping reviews: International Journal of EvidenceBased Healthcare, 13(3), 141-146.

https://doi.org/10.1097/XEB.0000000000000050

PEW. (2015). The Role of Emergency Savings in Family Financial Security. How Do Families Cope With Financial Shocks? The Pew Charitable Trusts.

https://www.pewtrusts.org/ /media/assets/2015/10/emergency-savings-report-

1_artfinal.pdf

PEW Charitable Trusts. (2015). How Do Families Cope With Financial Shocks? The Pew Charitable Trusts. https://www.pewtrusts.org/ /media/assets/.../emergency-savingsreport-1_artfinal.pdf

Popovic, N., Terzic-Supic, Z., Simic, S., \& Mladenovic, B. (2017). Predictors of unmet health care needs in Serbia; Analysis based on EU-SILC data. PLOS ONE, 12(11), e0187866. https://doi.org/10.1371/journal.pone.0187866

Pradhan, K. C., \& Mukherjee, S. (2018). Covariate and Idiosyncratic Shocks and Coping Strategies for Poor and Non-poor Rural Households in India. Journal of Quantitative Economics, 16(1), 101-127. https://doi.org/10.1007/s40953-017-0073-8

Preker, A., Carrin, G., Dror, D. M., Jakab, M., Hsiao, W., \& Arhin, D. (2007). Rich-Poor Differences in Health Care Financing (SSRN Scholarly Paper ID 1026850). Social Science Research Network. https://papers.ssrn.com/abstract $=1026850$

Quintal, C. (2019). Evolution of catastrophic health expenditure in a high income country: Incidence versus inequalities. International Journal for Equity in Health, 18(1), 1-11. https://doi.org/10.1186/s12939-019-1044-9

Raban, M. Z., Dandona, R., \& Dandona, L. (2013). Variations in catastrophic health expenditure estimates from household surveys in India. Bulletin of the World Health Organization, 91(10), 726-735. https://doi.org/10.2471/BLT.12.113100

Raine, R., Or, Z., Prady, S., \& Bevan, G. (2016). Evaluating health-care equity. In Challenges, solutions and future directions in the evaluation of service innovations in health care and public health. NIHR Journals Library. https://doi.org/10.3310/hsdr04160-69

Rapsomanikis, G. (2015). The economic lives of smallholder farmers (p. 48). Food and Agriculture Organization. http://www.fao.org/3/a-i5251e.pdf

Rasanathan, K., \& Diaz, T. (2016). Research on health equity in the SDG era: The urgent need for greater focus on implementation. International Journal for Equity in Health, 15(1). https://doi.org/10.1186/s12939-016-0493-7

Rashad, A., \& Sharaf, M. (2015). Catastrophic Economic Consequences of Healthcare Payments: Effects on Poverty Estimates in Egypt, Jordan, and Palestine. Economies, 3(4), 216 234. https://doi.org/10.3390/economies3040216

Rees, C. P., Hawkesworth, S., Moore, S. E., Dondeh, B. L., \& Unger, S. A. (2016). Factors Affecting Access to Healthcare: An Observational Study of Children under 5 Years of Age Presenting to a Rural Gambian Primary Healthcare Centre. PloS One, 11(6), e0157790. https://doi.org/10.1371/journal.pone.0157790 
Riddell, B. (2011). Africa's Informal Workers: Collective Agency, Alliances and Transnational Organizing in Urban Africa. African Studies Review, 54(3), 175-177.

Ro, Y. S., Shin, S. D., Jeong, J., Kim, M. J., Jung, Y. H., Kamgno, J., Alain, E. M. G., \& Hollong, B. (2017). Evaluation of demands, usage and unmet needs for emergency care in Yaounde, Cameroon: A cross-sectional study. Bmj Open, 7(2), e014573. https://doi.org/10.1136/bmjopen-2016-014573

Rodney, A. M., \& Hill, P. S. (2014). Achieving equity within universal health coverage: A narrative review of progress and resources for measuring success. Int J Equity Health, 13(1), 72.

Rosenbaum, P. R., \& Rubin, D. B. (1983). The central role of the propensity score in observational studies for causal effects. Biometrika, 70(1), 41-55. https://doi.org/10.1093/biomet/70.1.41

Roux, A. V. D. (2008). Next steps in understanding the multilevel determinants of health. Journal of Epidemiology and Community Health, 62(11), 957. https://doi.org/10.1136/jech.2007.064311

Russell, S. (2004). The economic burden of illness for households in developing countries: A review of studies focusing on malaria, tuberculosis, and human immunodeficiency virus/acquired immunodeficiency syndrome. The American Journal of Tropical Medicine and Hygiene, 71(2 suppl), 147-155.

Saksena, P., Hsu, J., \& Evans, D. B. (2014). Financial Risk Protection and Universal Health Coverage: Evidence and Measurement Challenges. Plos Medicine, 11(9), e1001701. https://doi.org/10.1371/journal.pmed.1001701

Saksena, P., Xu, K., \& Durairaj, V. (2010). The drivers of catastrophic expenditure: Outpatient services, hospitalization or medicines? (Background Paper No. 21). World Health Organisation. http://www.who.int/entity/healthsystems/topics/financing/healthreport/21whrbp.pdf?

Salari, P., Di Giorgio, L., Ilinca, S., \& Chuma, J. (2019). The catastrophic and impoverishing effects of out-of-pocket healthcare payments in Kenya, 2018. BMJ Global Health, 4(6). https://doi.org/10.1136/bmjgh-2019-001809

Sauerborn, R., Nougtara, A., Hien, M., \& Diesfeld, H. J. (1996). Seasonal variations of household costs of illness in Burkina Faso. Social Science \& Medicine, 43(3), 281-290. https://doi.org/10.1016/0277-9536(95)00374-6

Scheil-Adlung, X. (2014). Response to health inequity: The role of social protection in reducing poverty and achieving equity. Health Promotion International, 29(suppl_1), i59-i67. https://doi.org/10.1093/heapro/dau030

Séne, L. M., \& Cissé, M. (2015). Catastrophic out-of-pocket payments for health and poverty nexus: Evidence from Senegal. International Journal of Health Economics and Management, 15(3), 307-328. https://doi.org/10.1007/s10754-015-9170-4

Shi, L., \& Stevens, G. (2005). Vulnerability and unmet health care needs. The influence of multiple risk factors. Journal of General Internal Medicine, 20(2), 148-154. 
Shi, L., Stevens, G. D., Lebrun, L. A., Faed, P., \& Tsai, J. (2008). Enhancing the measurement of health disparities for vulnerable populations. Journal of Public Health Management and Practice, 14(6), S45-S52.

SID. (2004). Inequalities in Kenya: Pulling Apart-Facts and Figures on Inequality in Kenya. Society for International Development. http://sidint.net/docs/pullingapart-mini.pdf

Skinner, J., Chalkidou, K., \& Jamison, D. T. (2019). Valuing Protection against Health-Related Financial Risks. Journal of Benefit-Cost Analysis, 10(Suppl 1), 106-131. https://doi.org/10.1017/bca.2018.30

Sommet, N., \& Morselli, D. (2017). Keep Calm and Learn Multilevel Logistic Modeling: A Simplified Three-Step Procedure Using Stata, R, Mplus, and SPSS. International Review of Social Psychology, 30(1), 203-218. https://doi.org/10.5334/irsp.90

StataCorp. (2013). Stata Treatment-Effects Reference Manual: Potential outcomes/counterfactual outcomes: Release13. StataCorp LLP. https://www.stata.com/manuals13/te.pdf

StataCorp. (2019). Stata treatment-effects Reference manual: Potential outcomes/counterfactual outcomes: Release 16. StataCorp LLC. https://www.stata.com/manuals/te.pdf

Stuart, E. A. (2010). Matching methods for causal inference: A review and a look forward. Statistical Science : A Review Journal of the Institute of Mathematical Statistics, 25(1), 1-21. https://doi.org/10.1214/09-STS313

Su, T. T., Kouyaté, B., \& Flessa, S. (2006). Catastrophic household expenditure for health care in a low-income society: A study from Nouna District, Burkina Faso. Bulletin of the World Health Organization, 84(1), 21-27. https://doi.org//S0042-96862006000100010

Subramanian, S. V., Kawachi, I., \& Kennedy, B. P. (2001). Does the state you live in make a difference? Multilevel analysis of self-rated health in the US. Social Science \& Medicine, 53(1), 9-19. https://doi.org/10.1016/S0277-9536(00)00309-9

Suchman, L. (2018). Accrediting private providers with National Health Insurance to better serve low-income populations in Kenya and Ghana: A qualitative study. International Journal for Equity in Health, 17(1), 1-18. https://doi.org/10.1186/s12939-018-0893-y

Swamy, G. (1994). Kenya: Structural Adjustment in the 1980s. World Bank Publications.

Swe, K. T., Rahman, Md. M., Rahman, Md. S., Saito, E., Abe, S. K., Gilmour, S., \& Shibuya, K. (2018). Cost and economic burden of illness over 15 years in Nepal: A comparative analysis. PLOS ONE, 13(4), e0194564. https://doi.org/10.1371/journal.pone.0194564

Tanimura, T., Jaramillo, E., Weil, D., Raviglione, M., \& Lönnroth, K. (2014). Financial burden for tuberculosis patients in low- and middle-income countries: A systematic review. The European Respiratory Journal, 43(6), 1763-1775. https://doi.org/10.1183/09031936.00193413

Tey, N.-P., \& Lai, S. (2013). Correlates of and Barriers to the Utilization of Health Services for Delivery in South Asia and Sub-Saharan Africa. The Scientific World Journal, 2013, 1 11. https://doi.org/10.1155/2013/423403 
Thammatacharee, N., Tisayaticom, K., Suphanchaimat, R., Limwattananon, S., Putthasri, W., Netsaengtip, R., \& Tangcharoensathien, V. (2012). Prevalence and profiles of unmet healthcare need in Thailand. BMC Public Health, 12(1). https://doi.org/10.1186/14712458-12-923

Thavaneswaran, A. (2008). Propensity Score Matching in Observational Studies (p. 29). Manitoba Centre for Health Policy, University of Manitoba. https://www.umanitoba.ca/faculties/health_sciences/medicine/units/chs/department al_units/mchp/protocol/media/propensity_score_matching.pdf

Tomini, S. M., Packard, T. G., \& Tomini, F. (2013). Catastrophic and impoverishing effects of outof-pocket payments for health care in Albania: Evidence from Albania Living Standards Measurement Surveys 2002, 2005 and 2008. Health Policy and Planning, 28(4), 419428. https://doi.org/10.1093/heapol/czs073

Tran-Quang, V. (2012). Household behavior and post-shock recovery in Vietnam. 27.

Tsofa, B., Molyneux, S., Gilson, L., \& Goodman, C. (2017). How does decentralisation affect health sector planning and financial management? A case study of early effects of devolution in Kilifi County, Kenya. International Journal for Equity in Health, 16(1), 151. https://doi.org/10.1186/s12939-017-0649-0

Ughasoro, M. D., Onwujekwe, O. E., \& Ojinnaka, N. C. (2014). Economic cost of treatment of childhood epilepsy in Enugu, southeast Nigeria. International Journal of Technology Assessment in Health Care, 30(4), 469-474. https://doi.org/10.1017/S0266462314000518

Ukwaja, Kingsley N., Alobu, I., Lgwenyi, C., \& Hopewell, P. C. (2013). The High Cost of Free Tuberculosis Services: Patient and Household Costs Associated with Tuberculosis Care in Ebonyi State, Nigeria. PLOS ONE, 8(8), e73134. https://doi.org/10.1371/journal.pone.0073134

Ukwaja, Kingsley Nnanna, Alobu, I., Abimbola, S., \& Hopewell, P. C. (2013). Household catastrophic payments for tuberculosis care in Nigeria: Incidence, determinants, and policy implications for universal health coverage. Infectious Diseases of Poverty, 2, 21. https://doi.org/10.1186/2049-9957-2-21

University of Wisconsin. (2015). Propensity Score Matching in Stata using teffects. Supporting Statistical Analysis for Reseacrh. https://www.ssc.wisc.edu/sscc/pubs/stata_psmatch.htm

van Doorslaer, E., O’Donnell, O., Rannan-Eliya, R. P., Somanathan, A., Adhikari, S. R., Garg, C. C., Harbianto, D., Herrin, A. N., Huq, M. N., Ibragimova, S., Karan, A., Lee, T.-J., Leung, G. M., Lu, J.-F. R., Ng, C. W., Pande, B. R., Racelis, R., Tao, S., Tin, K., ... Zhao, Y. (2007). Catastrophic payments for health care in Asia. Health Economics, 16(11), 1159-1184. https://doi.org/10.1002/hec.1209

Van Minh, H., Kim Phuong, N. T., Saksena, P., James, C. D., \& Xu, K. (2013). Financial burden of household out-of pocket health expenditure in Viet Nam: Findings from the National Living Standard Survey 2002-2010. Social Science \& Medicine, 96, 258-263. https://doi.org/10.1016/j.socscimed.2012.11.028 
VanderWeele, T. J., \& Ding, P. (2017). Sensitivity Analysis in Observational Research: Introducing the E-Value. Annals of Internal Medicine, 167(4), 268. https://doi.org/10.7326/M162607

Vedom, J., \& Cao, H. (2011). Health care access and regional disparities in China. Space, Population and Societies. http://citeseerx.ist.psu.edu/viewdoc/download?doi=10.1.1.900.550\&rep=rep1\&type= pdf

Verguet, S., Memirie, S. T., \& Norheim, O. F. (2016). Assessing the burden of medical impoverishment by cause: A systematic breakdown by disease in Ethiopia. BMC Medicine, 14. https://doi.org/10.1186/s12916-016-0697-0

Wagstaff, A., van Doorslaer, E., \& Watanabe, N. (2003). On decomposing the causes of health sector inequalities with an application to malnutrition inequalities in Vietnam. Journal of Econometrics, 112(1), 207-223. https://doi.org/10.1016/S0304-4076(02)00161-6

Wagstaff, Adam. (2005). The Economic Consequences Of Health Shocks. The World Bank. https://doi.org/10.1596/1813-9450-3644

Wagstaff, Adam. (2009). Correcting the concentration index: A comment. Journal of Health Economics, 28(2), 516-520. https://doi.org/10.1016/j.jhealeco.2008.12.003

Wagstaff, Adam, \& Doorslaer, E. van. (2003). Catastrophe and impoverishment in paying for health care: With applications to Vietnam 1993-1998. Health Economics, 12(11), 921-933.

Wagstaff, Adam, \& Eozenou, P. H.-V. (2014). CATA Meets IMPOV: A Unified Approach to Measuring Financial Protection in Health. The World Bank. https://doi.org/10.1596/1813-9450-6861

Wagstaff, Adam, Flores, G., Hsu, J., Smitz, M.-F., Chepynoga, K., Buisman, L. R., van Wilgenburg, K., \& Eozenou, P. (2018). Progress on catastrophic health spending in 133 countries: A retrospective observational study. The Lancet Global Health, 6(2), e169-e179. https://doi.org/10.1016/S2214-109X(17)30429-1

Wang, L., Wang, A., Zhou, D., FitzGerald, G., Ye, D., \& Jiang, Q. (2016). An Empirical Analysis of Rural-Urban Differences in Out-Of-Pocket Health Expenditures in a Low-Income Society of China. PLoS ONE, 11(5). https://doi.org/10.1371/journal.pone.0154563

Wang, Q., Brenner, S., Kalmus, O., Banda, H. T., \& De Allegri, M. (2016). The economic burden of chronic non-communicable diseases in rural Malawi: An observational study. BMC Health Services Research, 16, 457. https://doi.org/10.1186/s12913-016-1716-8

Wang, Q., Fu, A. Z., Brenner, S., Kalmus, O., Banda, H. T., \& De Allegri, M. (2015). Out-of-pocket expenditure on chronic non-communicable diseases in sub-Saharan Africa: The case of rural Malawi. PloS One, 10(1), e0116897. https://doi.org/10.1371/journal.pone.0116897

Wardle, J., \& Steel, A. (2015). Systematic reviews in integrative medicine: A clinician's guide to publication. Advances in Integrative Medicine, 2(2), 103-109. https://doi.org/10.1016/j.aimed.2015.09.001 
Waters, H. R. (2000). Measuring equity in access to health care. Social Science \& Medicine, 51(4), 599-612. https://doi.org/10.1016/S0277-9536(00)00003-4

Weinstein, J. N., Geller, A., Negussie, Y., \& Baciu, A. (2017). Communities in Action: Pathways to Health Equity (Committee on Community-Based Solutions to Promote Health Equity in the United States, Board on Population Health and Public Health Practice, Health and Medicine Division, \& National Academies of Sciences, Engineering, and Medicine, Eds.). National Academies Press. https://doi.org/10.17226/24624

Welch, V. A., Petticrew, M., O’Neill, J., Waters, E., Armstrong, R., Bhutta, Z. A., Francis, D., Koehlmoos, T. P., Kristjansson, E., Pantoja, T., \& others. (2013). Health equity: Evidence synthesis and knowledge translation methods. Systematic Reviews, 2(1), 43.

Welch, V., Tugwell, P., Petticrew, M., de Montigny, J., Ueffing, E., Kristjansson, B., McGowan, J., Benkhalti Jandu, M., Wells, G. A., Brand, K., \& Smylie, J. (2010). How effects on health equity are assessed in systematic reviews of interventions. In The Cochrane Collaboration (Ed.), Cochrane Database of Systematic Reviews. John Wiley \& Sons, Ltd. https://doi.org/10.1002/14651858.MR000028.pub2

WHO. (2005). Designing health financing systems to reduce catastrophic health expenditure (Technical Brief Number 2-5). World Health Organisation. http://apps.who.int/iris/bitstream/10665/70005/1/WHO_EIP_HSF_PB_05.02_eng.p df

WHO. (2006). The world health report: Arguing for Universal Health Coverage. http://apps.who.int/iris/handle/10665/43432

WHO. (2007). Coping with out-of-pocket health payments: Applications of Engel curves and twopart models in six African countries. http://apps.who.int/iris/handle/10665/85677

WHO. (2010). A conceptual framework for action on the social determinants of health: Debates, policy \& practice, case studies. http://apps.who.int/iris/bitstream/10665/44489/1/9789241500852_eng.pdf

WHO (Ed.). (2013). Handbook on health inequality monitoring: With a special focus on low- and middle-income countries. World Health Organization.

WHO. (2015). Health 2020: Social protection and health. World Health Organisation, Regional Office for Europe.

WHO. (2017). Together on the road to universal health coverage: A call to action. World Health Organization. https://apps.who.int/iris/bitstream/handle/10665/258962/WHO-HISHGF-17.1-eng.pdf;jsessionid=BCA55D1E3EFBEE631878A92E2148A185?sequence=1

WHO, Etienne, C., Asamoa-Baah, A., \& Evans, D. B. (Eds.). (2010). The World health report: Health systems financing: the path to universal coverage. World Health Organization.

WHO, \& World Bank. (2017). Tracking universal health coverage: 2017 global monitoring report (No. 122029; pp. 1-88). The World Bank. http://documents.worldbank.org/curated/en/640121513095868125/Trackinguniversal-health-coverage-2017-global-monitoring-report

Williamson, T., \& Mulaki, A. (2015). Devolution of Kenya's health system: The role of health Policy project. RTI International. 
Witter, S., Adjei, S., Armar-Klemesu, M., \& Graham, W. (2009). Providing free maternal health care: Ten lessons from an evaluation of the national delivery exemption policy in Ghana. Global Health Action, 2(0). http://www.globalhealthaction.net/index.php/gha/article/view/1881

Woolf, S. H., Simon, S., Aron, L., Emily Zimmerman, Dubay, L., \& Luk, K. (2015). How Are Income and Wealth Linked to Health and Longevity? Urban Institute and Virginia Commonwealth University, 22.

World Bank. (2000). World Development Report 2000/2001:Attacking Poverty. World Bank. http://documents.worldbank.org/curated/en/230351468332946759/pdf/226840W DROOPUBOng0poverty0200002001.pdf

World Bank. (2012). Devolution without disruption:Pathways to a successful new Kenya.

World Bank. (2013). World Development Report 2014: Risk and Opportunity-Managing Risk for Development (p. 363). International Bank for Reconstruction and Development / The World Bank.

World Bank. (2014). Laying The Foundation For A Robust Health Care System In Kenya. Kenya Public Expenditure Review. The World Bank.

Xu, K. (2005). Distribution of health payments and catastrophic expenditures methodology [Number 2 - 2005]. World Health Organisation. http://apps.who.int/iris/bitstream/10665/69030/1/EIP_HSF_DP_05.2.pdf

Xu, K., Evans, D. B., Carrin, G., Aguilar-Rivera, A. M., Musgrove, P., \& Evans, T. (2007). Protecting Households From Catastrophic Health Spending. Health Affairs, 26(4), 972-983. https://doi.org/10.1377/hlthaff.26.4.972

Xu, K., Evans, D. B., Kadama, P., Nabyonga, J., Ogwal, P. O., Nabukhonzo, P., \& Aguilar, A. M. (2006). Understanding the impact of eliminating user fees: Utilization and catastrophic health expenditures in Uganda. Social Science \& Medicine (1982), 62(4), 866-876. https://doi.org/10.1016/j.socscimed.2005.07.004

Xu, K., Evans, D. B., Kawabata, K., Zeramdini, R., Klavus, J., \& Murray, C. J. (2003a). Household catastrophic health expenditure: A multicountry analysis. The Lancet, 362(9378), 111117. https://doi.org/10.1016/S0140-6736(03)13861-5

Xu, K., Evans, D. B., Kawabata, K., Zeramdini, R., Klavus, J., \& Murray, C. J. (2003b). Household catastrophic health expenditure: A multicountry analysis. The Lancet, 362(9378), 111117. https://doi.org/10.1016/S0140-6736(03)13861-5

Xu, K., James, C., Carrin, G., \& Muchiri, S. (2006). An empirical model of access to health care, health care expenditure and impoverishment in Kenya: Learning from past reforms and lessons for the future (Discussion Paper NUMBER 3-2006). World Health Organisation. http://www.who.int/health_financing/documents/cov-dp_06_3_access_kenya/en/

Yang, B.-M. (2001). The impact of economic crisis on health-care consumption in Korea. Health Policy and Planning, 16(4), 372-385. https://doi.org/10.1093/heapol/16.4.372

Yu, C., Whynes, D. K., \& Sach, T. H. (2008). Equity in health care financing: The case of Malaysia. International Journal for Equity in Health, 7(1), 15. https://doi.org/10.1186/14759276-7-15 
Zere, E., \& McIntyre, D. (2003). Equity in self-reported adult illness and use of health service in South Africa: Inter-temporal comparison. Journal of Health, Population and Nutrition, 205-215.

Zhehui Luo, Gardiner, J. C., \& Bradley, C. J. (2010). Applying Propensity Score Methods in Medical Research: Pitfalls and Prospects. Medical Care Research and Review, 67(5), 528-554. https://doi.org/10.1177/1077558710361486

Zimmerman, E., \& Woolf, S. H. (2014). Understanding the Relationship Between Education and Health. NAM Perspectives, 4(6). https://doi.org/10.31478/201406a

Zollmann, J., \& Ravishankar, N. (2016). Struggling to thrive: How Kenya's low-income families (try to) pay for healthcare (p. 40). Kenya Financial Sector Deepening(FSD). https://fsdkenya.org/publication/struggling-to-thrive-how-kenyas-low-income-familiestry-to-pay-for-healthcare/

Zon, H., Pavlova, M., \& Groot, W. (2019). Decentralization and health resources transfer to local governments in Burkina Faso: A SWOT analysis among health care decision makers. Health Science Reports, 2(6), e119. https://doi.org/10.1002/hsr2.119 


\section{Appendices}

Appendix 2-A: Search string for various databases

PUBMED
(((((((catastrophic) OR impoverishment) OR financial burden) OR economic burden) AND expenditure,
health[MeSH Terms]) OR cost, healthcare[MeSH Terms]) AND africa south of the sahara[MeSH Terms])
AND full text[sb] AND "last 10 years"[PDat] AND Humans[Mesh] AND English[lang])
(((((((((catastrophic[Title/Abstract]) OR impoverishment[Title/Abstract]) OR financial
burden[Title/Abstract]) AND cost, healthcare[MeSH Terms]) OR expenditure, health [MeSH Terms]) AND
africa south of the sahara[MeSH Terms]) AND full text[sb] AND "last 10 years"[PDat] AND
Humans[Mesh] AND English[lang]))))
JSTOR
((((((Catastrophic) OR (impoverish*)) OR ("economic burden")) OR ("financial burden")) AND ("health
expenditure")) OR ("healthcare cost*")) AND (Africa)) AND la:(eng OR en) AND disc:(healthsciences-
discipline OR health-discipline)
SUBJECT: Health policy; Health Science
Article type - Articles ; Reviews; Books; Research Reports
EBSCO host
TX catastrophic OR TX impoverish* OR TX "financial burden" OR TX "economic burden" AND TX "health
expenditure" OR TX "healthcare cost" AND TX Africa
CINAHL, Econlit, PsycINFO
Limiters - Published Date: 20060101-20170531
Narrow by Language: - english
Narrow by Subject: - health: government policy; regulation; public health
Search modes - Boolean/Phrase
\#\#\#\#\#No limiters per journal
WEB OF SCIENCE


Appendix 2-B: Quality assessment tool for observational cohort and cross-sectional studies

\begin{tabular}{|c|c|c|c|}
\hline \multirow[t]{2}{*}{ Criteria } & \multicolumn{3}{|c|}{ No. of articles } \\
\hline & Yes & No & $\begin{array}{l}\text { Other } \\
(C D, N R \text {, } \\
\text { NA)* }\end{array}$ \\
\hline 1. Was the research question or objective in this paper clearly stated? & 34 & & \\
\hline 2. Was the study population clearly specified and defined? & 34 & & \\
\hline 3. Was the participation rate of eligible persons at least $50 \%$ ? & 33 & & $1 *$ \\
\hline $\begin{array}{l}\text { 4. Were all the subjects selected or recruited from the same or similar } \\
\text { populations (including the same time period)? Were inclusion and } \\
\text { exclusion criteria for being in the study pre-specified and applied } \\
\text { uniformly to all participants? }\end{array}$ & 33 & & $1^{*}$ \\
\hline $\begin{array}{l}\text { 5. Was a sample size justification, power description, or variance and } \\
\text { effect estimates provided? }\end{array}$ & 33 & & $1 *$ \\
\hline $\begin{array}{l}\text { 6. For the analyses in this paper, were the exposure(s) of interest } \\
\text { measured prior to the outcome(s) being measured? }\end{array}$ & 18 & & 16 \\
\hline $\begin{array}{l}\text { 7. Was the timeframe sufficient so that one could reasonably expect } \\
\text { to see an association between exposure and outcome if it existed? }\end{array}$ & & & 34 \\
\hline $\begin{array}{l}\text { 8. For exposures that can vary in amount or level, did the study } \\
\text { examine different levels of the exposure as related to the outcome } \\
\text { (e.g., categories of exposure, or exposure measured as continuous } \\
\text { variable)? }\end{array}$ & 11 & 6 & 17 \\
\hline $\begin{array}{l}\text { 9. Were the exposure measures (independent variables) clearly } \\
\text { defined, valid, reliable, and implemented consistently across all } \\
\text { study participants? }\end{array}$ & 18 & & 16 \\
\hline 10. Was the exposure(s) assessed more than once over time? & & & 34 \\
\hline $\begin{array}{l}\text { 11. Were the outcome measures (dependent variables) clearly } \\
\text { defined, valid, reliable, and implemented consistently across all } \\
\text { study participants? }\end{array}$ & 34 & & \\
\hline $\begin{array}{l}\text { 12. Were the outcome assessors blinded to the exposure status of } \\
\text { participants? }\end{array}$ & & & 34 \\
\hline 13. Was loss to follow-up after baseline $20 \%$ or less? & & & 34 \\
\hline $\begin{array}{l}\text { 14. Were key potential confounding variables measured and } \\
\text { adjusted statistically for their impact on the relationship between } \\
\text { exposure(s) and outcome(s)? }\end{array}$ & 18 & & 16 \\
\hline
\end{tabular}

${ }^{\star} \mathrm{CD}$, cannot determine; NA, not applicable; NR, not reported 


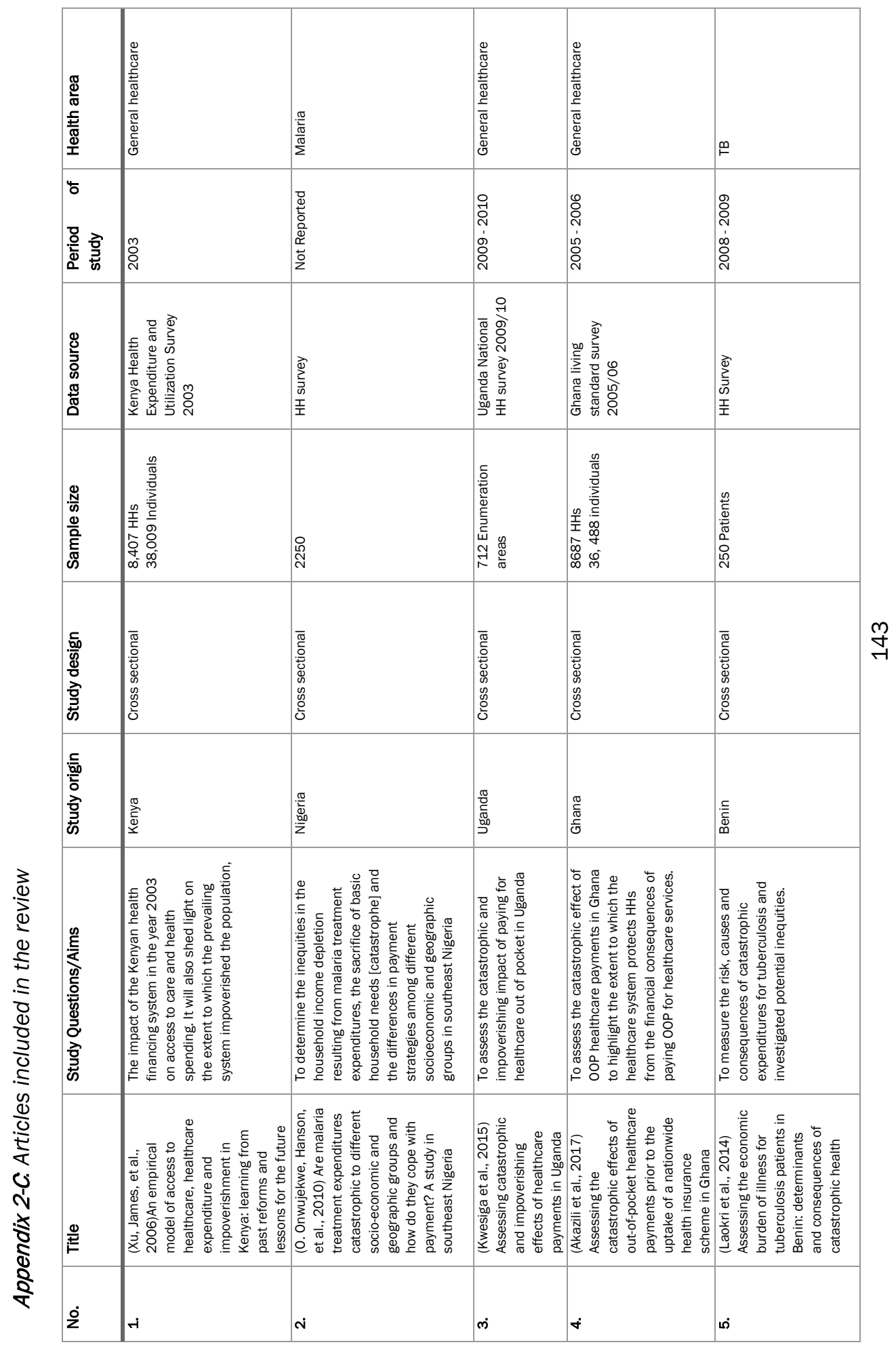




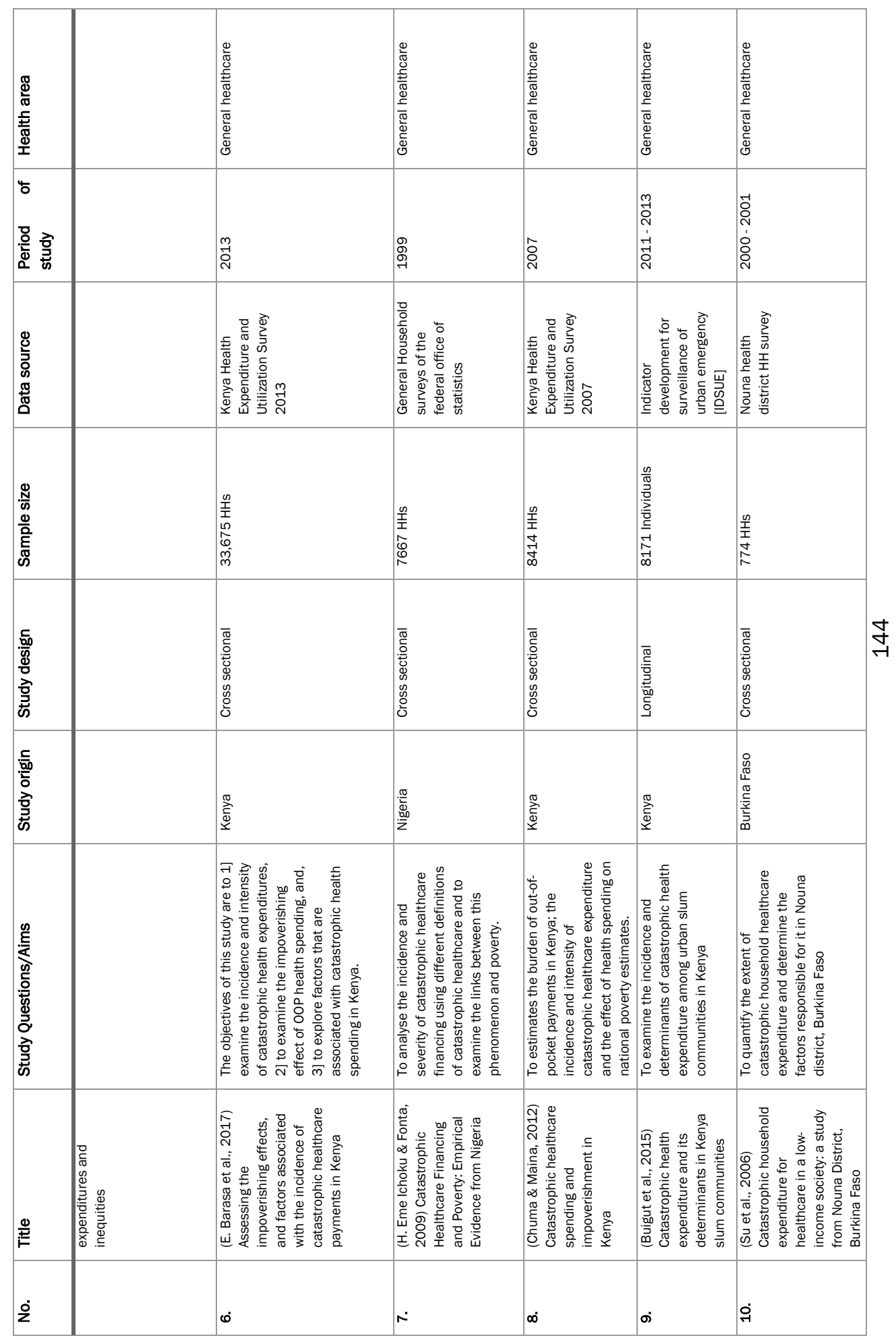




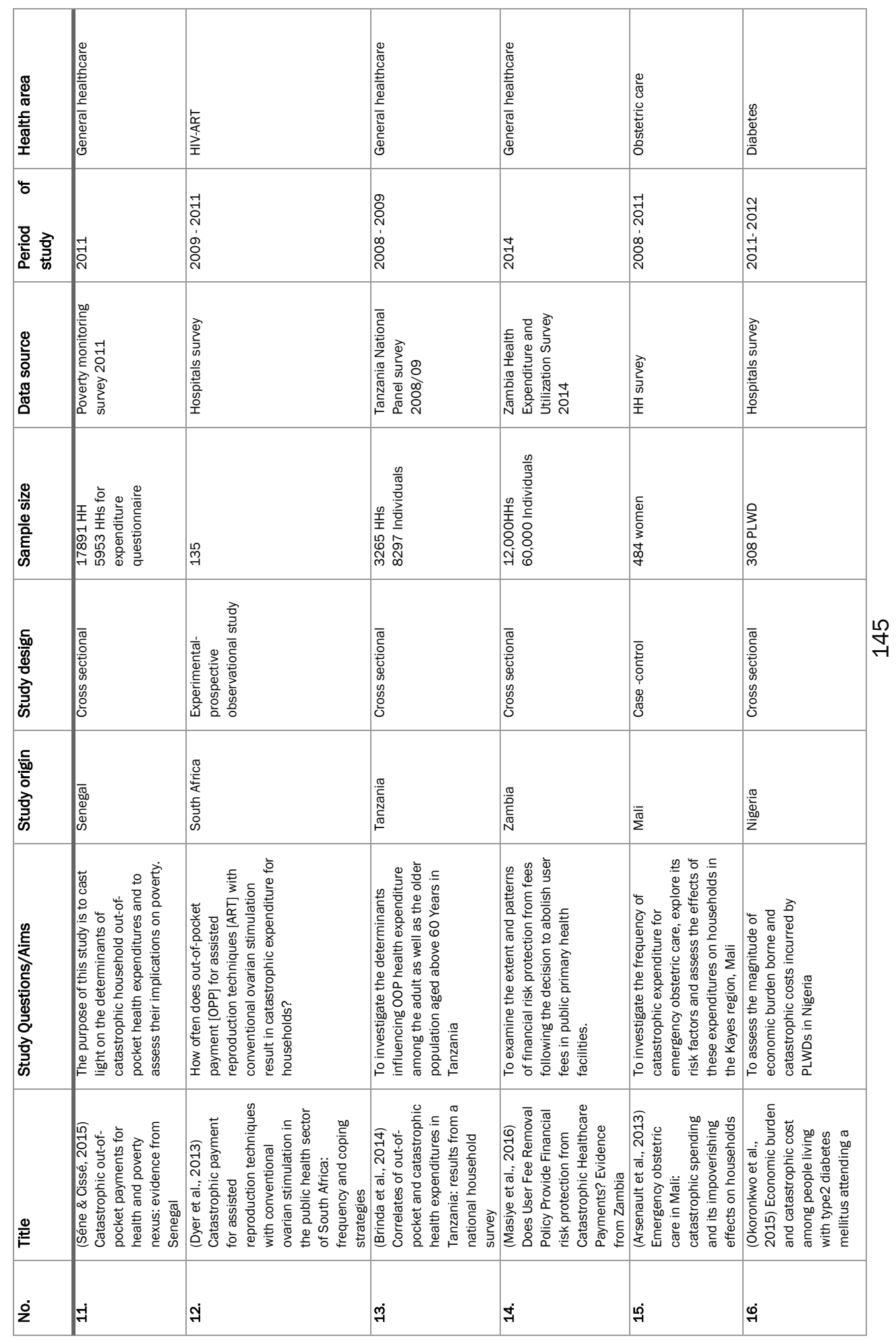




\begin{tabular}{|c|c|c|c|c|c|}
\hline 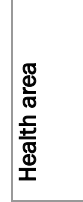 & & 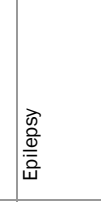 & 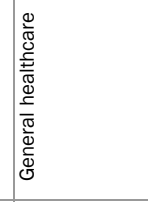 & 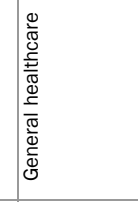 & 锃 \\
\hline 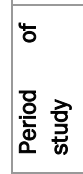 & & त् & 兽 & 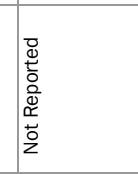 & 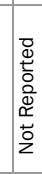 \\
\hline 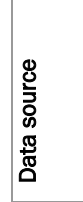 & & 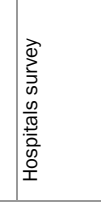 & 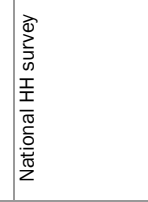 & $\begin{array}{l}\text { 产 } \\
\text { 产 } \\
\text { 产 }\end{array}$ & 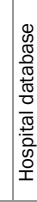 \\
\hline 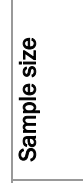 & & 声 & & 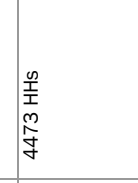 & 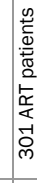 \\
\hline 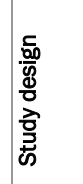 & & 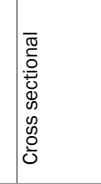 & & 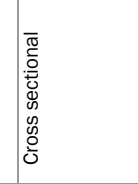 & 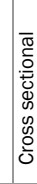 \\
\hline 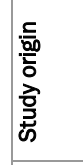 & & 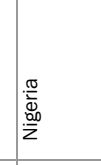 & 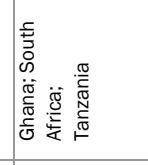 & 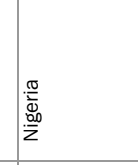 & 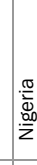 \\
\hline 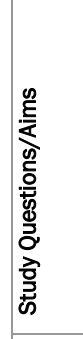 & & 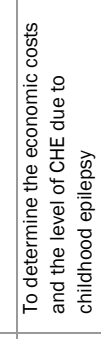 & 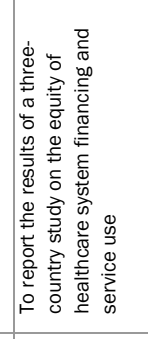 & 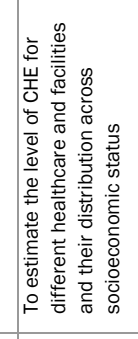 & \\
\hline$\stackrel{\varrho}{\frac{\vec{F}}{F}}$ & 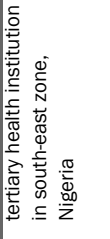 & 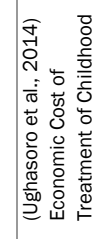 & 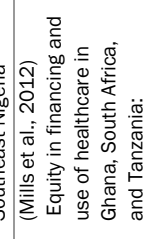 & 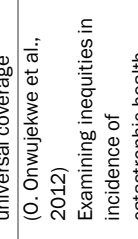 & \\
\hline$\dot{q}$ & & $\hat{A}$ & $\overbrace{-1}^{\infty}$ & $\stackrel{g}{g}$ & i் \\
\hline
\end{tabular}

$\stackrel{0}{\sim}$ 


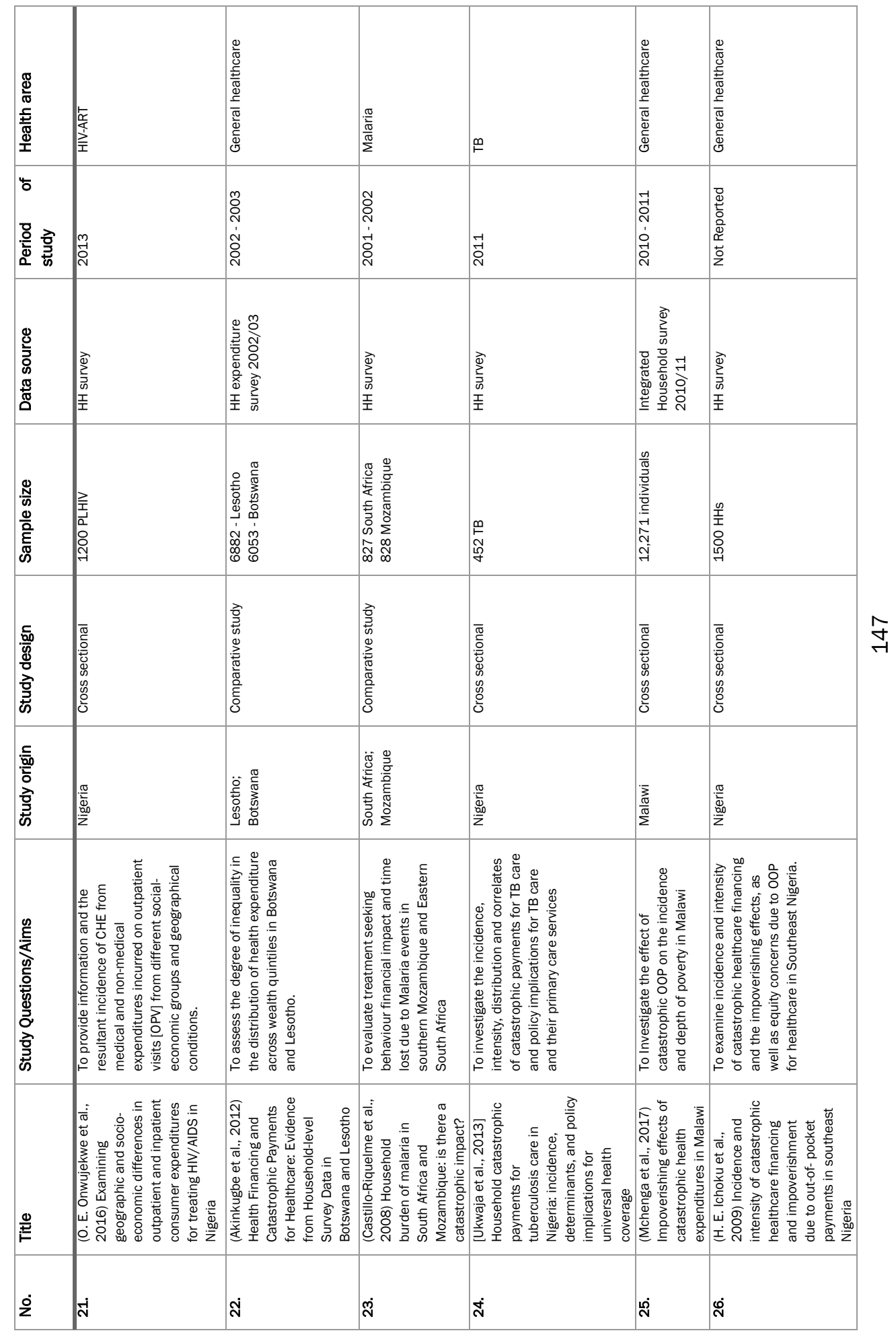




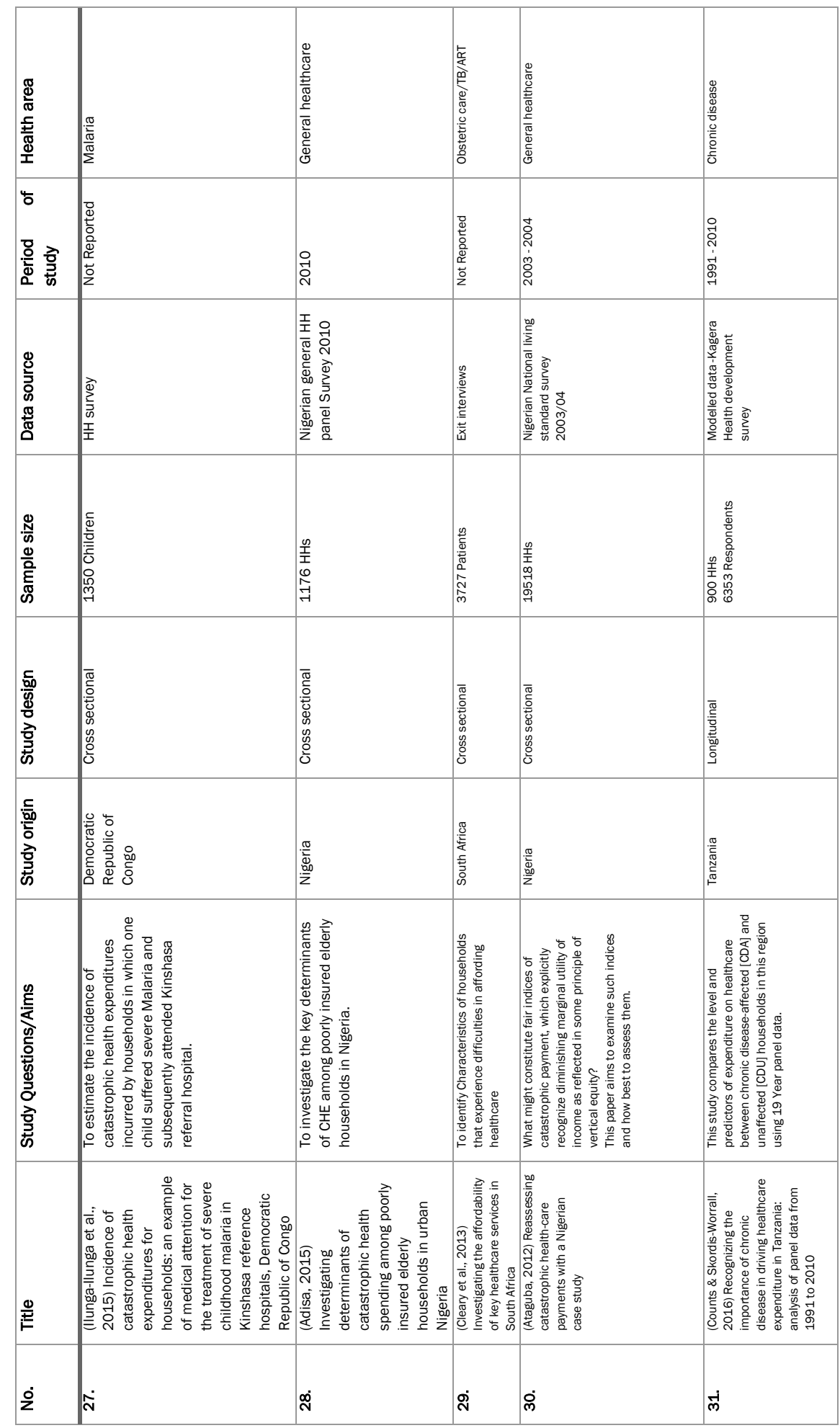

$\stackrel{\infty}{+}$ 


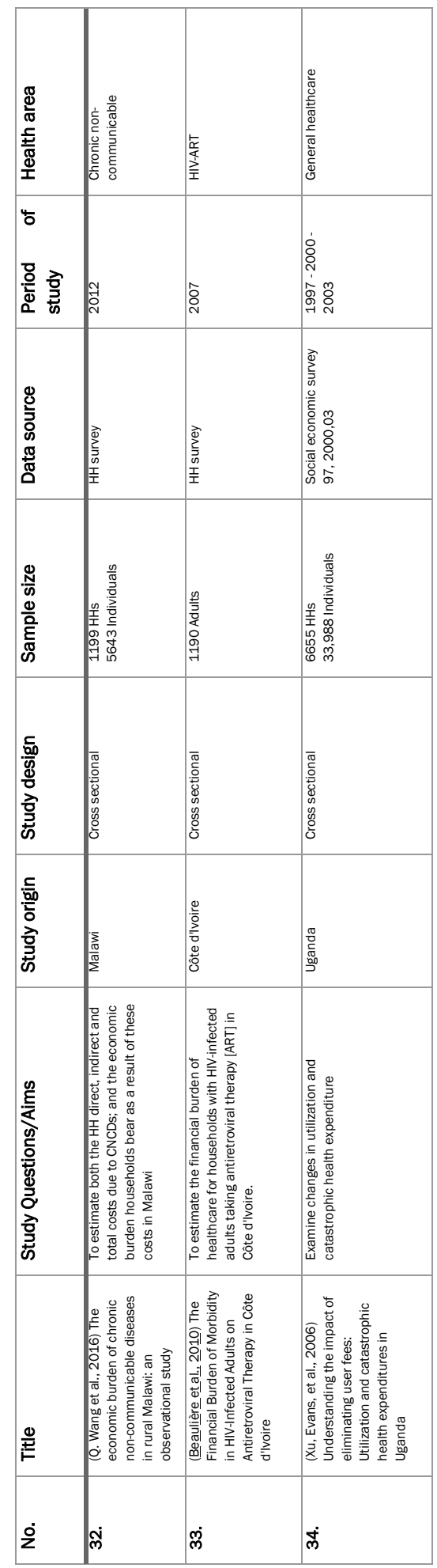

g 


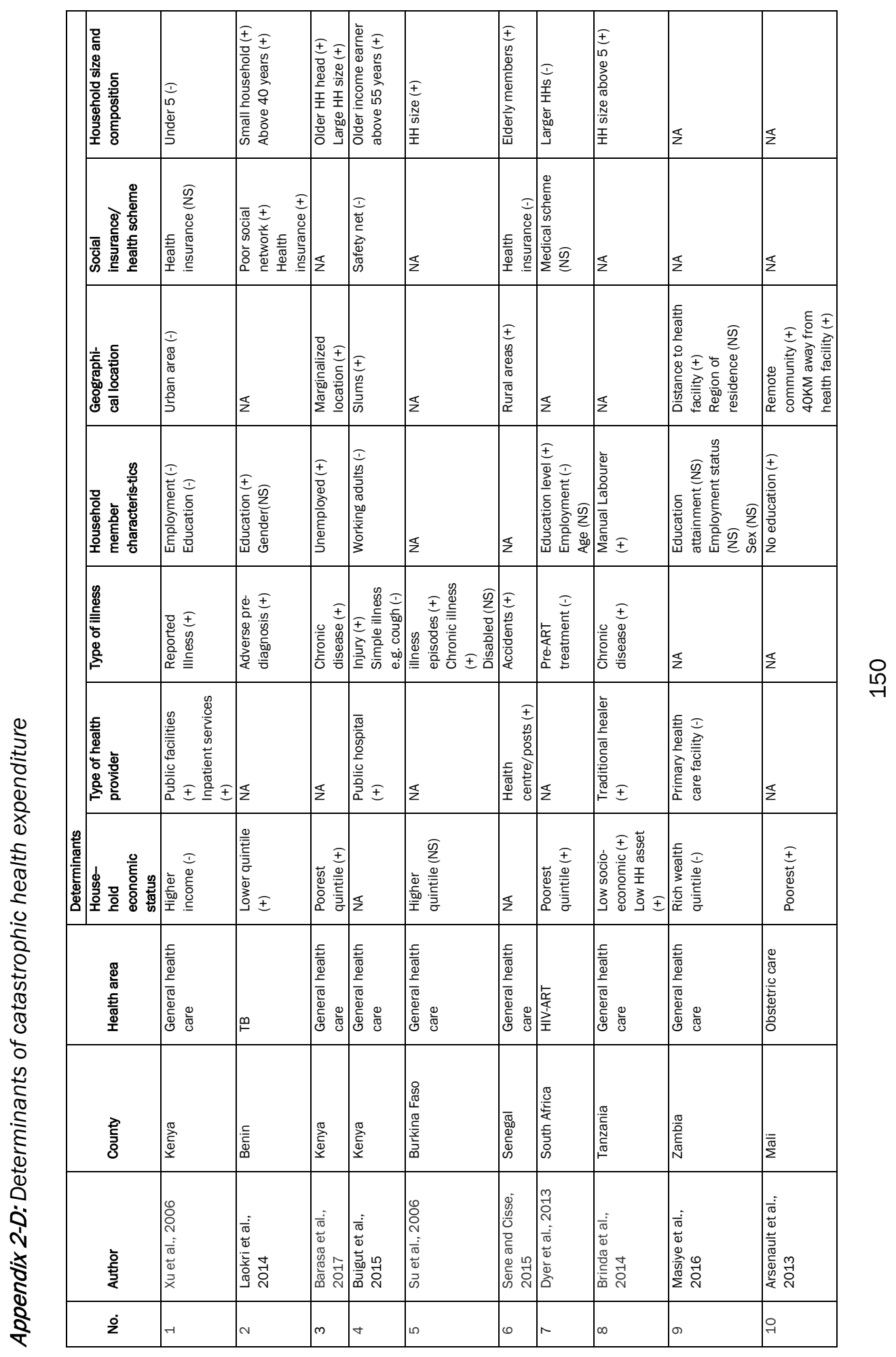




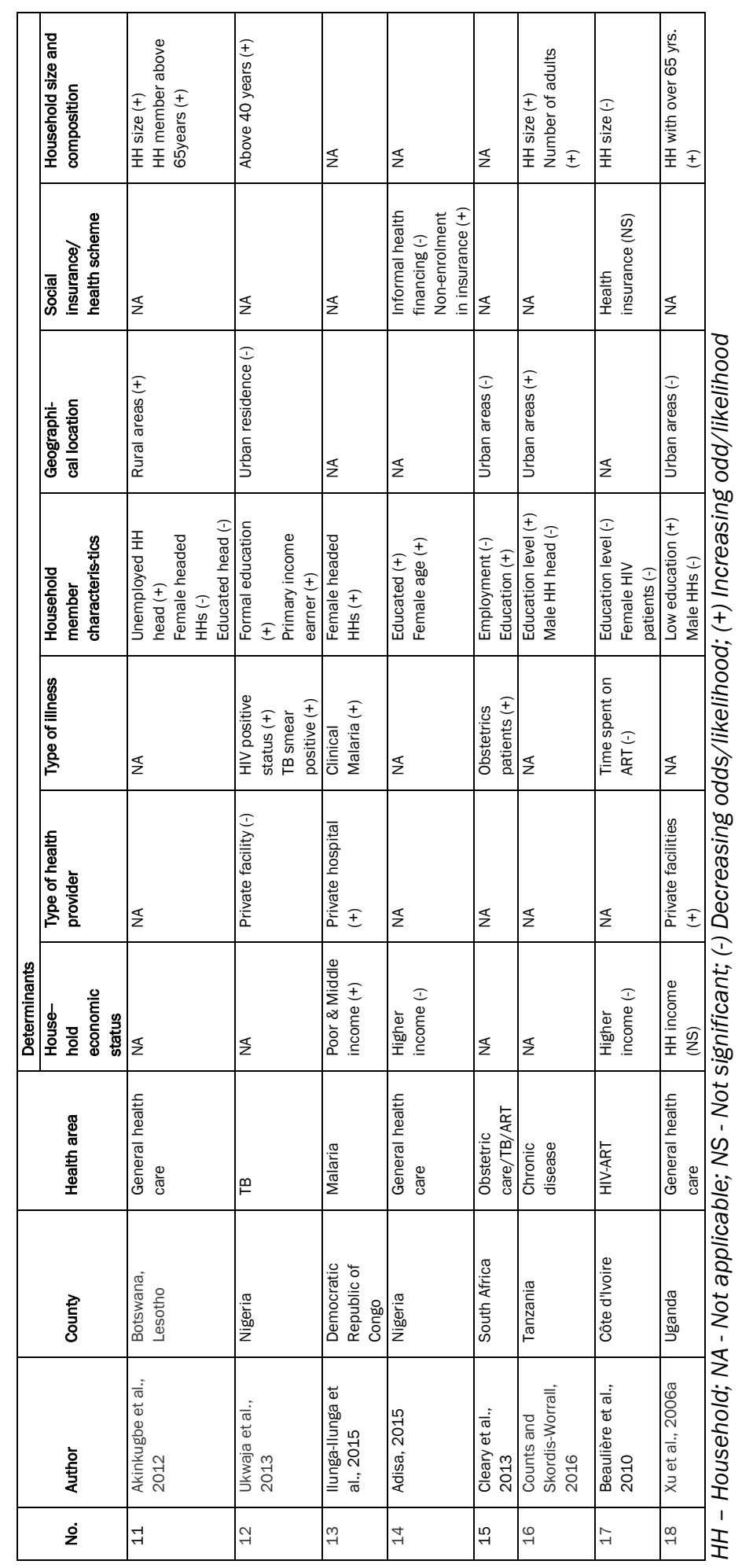

点 
Appendix 3-A: Households that experienced Catastrophic Health Expenditure (CHE) by Wealth Quintiles, 2007 and 2013

\begin{tabular}{|c|c|c|c|c|c|c|c|c|}
\hline \multirow{2}{*}{ Description } & \multirow{2}{*}{ Year } & \multicolumn{2}{|c|}{$\begin{array}{l}\text { Households with } \\
\text { CHE }\end{array}$} & \multicolumn{2}{|c|}{$\begin{array}{l}\text { Incidence difference } \\
(2013-2007)\end{array}$} & \multicolumn{2}{|c|}{$\begin{array}{l}\text { Households without } \\
\mathrm{CHE}\end{array}$} & \multirow{2}{*}{$\begin{array}{l}\text { Total } \\
\text { Cases(N) }\end{array}$} \\
\hline & & $\begin{array}{l}\text { Cases } \\
(\mathrm{N})\end{array}$ & $\begin{array}{l}\text { Percent* } \\
(\%)\end{array}$ & $\begin{array}{l}\text { Absolute } \\
\text { diff. }\end{array}$ & $\begin{array}{l}\text { Percent } \\
\text { diff.(\%) }\end{array}$ & $\begin{array}{l}\text { Cases } \\
(\mathrm{N})\end{array}$ & $\begin{array}{l}\text { Percent } \\
\text { (\%) }\end{array}$ & \\
\hline \multirow{2}{*}{$\begin{array}{l}\text { Total } \\
\text { (All quintiles) }\end{array}$} & 2007 & 427 & 11.4 & \multirow{2}{*}{-4.89} & \multirow{2}{*}{42.7} & 3,301 & 88.6 & 3,728 \\
\hline & 2013 & 1,084 & 6.5 & & & 15,442 & 93.4 & 16,526 \\
\hline \multirow{2}{*}{ Richest Quintile } & 2007 & 37 & 4.9 & \multirow{2}{*}{-3.09} & \multirow{2}{*}{63.3} & 721 & 95.1 & 758 \\
\hline & 2013 & 68 & 1.8 & & & 3,723 & 98.2 & 3,791 \\
\hline \multirow{2}{*}{ Fourth Quintile } & 2007 & 55 & 7.2 & \multirow{2}{*}{-4.55} & \multirow{2}{*}{63.5} & 712 & 92.8 & 767 \\
\hline & 2013 & 92 & 2.6 & & & 3,420 & 97.4 & 3,512 \\
\hline \multirow{2}{*}{ Middle Quintile } & 2007 & 74 & 9.8 & \multirow{2}{*}{-4.99} & \multirow{2}{*}{50.9} & 680 & 90.2 & 754 \\
\hline & 2013 & 152 & 4.8 & & & 3,001 & 95.2 & 3,153 \\
\hline \multirow{2}{*}{ Second Quintile } & 2007 & 99 & 12.8 & \multirow{2}{*}{-5.64} & \multirow{2}{*}{44.0} & 673 & 87.2 & 772 \\
\hline & 2013 & 225 & 7.2 & & & 2,909 & 92.8 & 3,134 \\
\hline \multirow[t]{2}{*}{ Poorest Quintile } & 2007 & 162 & 23.9 & \multirow{2}{*}{-5.30} & \multirow{2}{*}{22.1} & 515 & 76.1 & 677 \\
\hline & 2013 & 547 & 18.6 & & & 2,389 & 81.4 & 2,936 \\
\hline
\end{tabular}

*Incidence of catastrophic health expenditure (CHE) at 40\% threshold; diff: Difference 
Appendix 3-B: Multivariate regression model for determinants of Catastrophic Health Expenditure (CHE), 2007 and 2013

\begin{tabular}{|c|c|c|c|c|}
\hline \multirow[b]{2}{*}{ Variables } & \multicolumn{2}{|l|}{2007} & \multicolumn{2}{|l|}{2013} \\
\hline & Odds Ratio & $\begin{array}{l}{[95 \%} \\
\text { Interval] }\end{array}$ & Odds Ratio & $\begin{array}{l}{[95 \%} \\
\text { Interval] }\end{array}$ \\
\hline \multicolumn{5}{|l|}{$\begin{array}{l}\text { Wealth status (Ref. } \\
\text { Poorest) }\end{array}$} \\
\hline Second quintile & $0.454 * * *$ & $(0.321-0.644)$ & $0.282 * * *$ & $(0.228-0.484)$ \\
\hline Middle quintile & $0.284 * * *$ & $(0.190-0.425)$ & $0.159 * * *$ & $(0.106-0.255)$ \\
\hline Fourth quintile & $0.182 * * *$ & $(0.112-0.295)$ & $0.063 * * *$ & $(0.057-0.143)$ \\
\hline Richest quintile & $0.105 * * *$ & $(0.055-0.200)$ & $0.025 * * *$ & $(0.015-0.050)$ \\
\hline \multicolumn{5}{|l|}{$\begin{array}{l}\text { Gender of household head } \\
\text { (Ref. Male) }\end{array}$} \\
\hline Female & $0.656 * *$ & $(0.473-0.908)$ & 1.005 & $(0.748-1.350)$ \\
\hline \multicolumn{5}{|l|}{$\begin{array}{l}\text { Age of household head } \\
\text { (Ref. Below } 25 \text { years) }\end{array}$} \\
\hline $25-40$ years & 1.311 & $(0.635-2.707)$ & 1.258 & $(0.612-2.585)$ \\
\hline $40+$ years & 1.205 & $(0.574-2.526)$ & 0.860 & $(0.406-1.818)$ \\
\hline \multicolumn{5}{|c|}{$\begin{array}{l}\text { Education level of the household head } \\
\text { (Ref. No Education) }\end{array}$} \\
\hline Primary Education & 0.764 & $(0.546-1.069)$ & 1.449 & $(0.836-2.510)$ \\
\hline Secondary Education & 0.744 & $(0.468-1.184)$ & 1.150 & $(0.588-2.246)$ \\
\hline Tertiary Education & 0.772 & $(0.342-1.742)$ & 2.203 & $(0.955-5.083)$ \\
\hline $\begin{array}{l}\text { Employment status of the } \mathrm{H} \\
\text { (Ref. Unemployed } \mathrm{HH} \text { head) }\end{array}$ & head & & & $\cdot$ \\
\hline Employed $\mathrm{HH}$ head & $0.600 * * *$ & $(0.445-0.808)$ & $0.703 * *$ & $(0.512-0.963)$ \\
\hline \multicolumn{5}{|l|}{$\begin{array}{l}\text { Household wt. U5 Children } \\
\text { (Ref. Above } 5 \text { years) }\end{array}$} \\
\hline Less than $5(<5)$ years & 0.896 & $(0.642-1.251)$ & 0.882 & $(0.591-1.317)$ \\
\hline \multicolumn{5}{|l|}{$\begin{array}{l}\text { Household wt. Elderly } \\
\text { (Ref. }<60 \text { years) }\end{array}$} \\
\hline $60+$ years & 1.174 & $(0.839-1.643)$ & $1.586 * *$ & $(1.090-2.308)$ \\
\hline \multicolumn{5}{|l|}{$\begin{array}{l}\text { Household size } \\
\text { (Ref. 1-3 Small HH) }\end{array}$} \\
\hline 4-6 Medium $\mathrm{HH}$ & 0.742 & $(0.528-1.043)$ & $0.508 * * *$ & $(0.354-0.730)$ \\
\hline 7+ Large $\mathrm{HH}$ & 1.001 & $(0.648-1.546)$ & $0.432 * * *$ & $(0.248-0.754)$ \\
\hline \multicolumn{5}{|l|}{$\begin{array}{l}\text { Residence (Ref. Rural } \\
\text { residence) }\end{array}$} \\
\hline Urban Residence & 0.815 & $(0.543-1.223)$ & 0.976 & $(0.715-1.333)$ \\
\hline \multicolumn{5}{|l|}{$\begin{array}{l}\text { Insurance status } \\
\text { (Ref. Not insured) }\end{array}$} \\
\hline Insured Households & 1.263 & $(0.883-1.805)$ & $2.558 * * *$ & $(1.734-3.773)$ \\
\hline \multicolumn{5}{|l|}{$\begin{array}{l}\text { Type of health provider } \\
\text { (Ref. Public) }\end{array}$} \\
\hline Private provider & $1.694 * * *$ & $(1.260-2.277)$ & $2.903 * * *$ & $(2.131-3.954)$ \\
\hline Other providers & 0.705 & $(0.458-1.087)$ & 0.737 & $(0.478-1.136)$ \\
\hline \multicolumn{5}{|c|}{$\begin{array}{l}\text { HH wt. member with chronic illness } \\
\text { (Ref. No chronic illness) }\end{array}$} \\
\hline Member with Chronic illness & $1.377 * *$ & $(1.025-1.849)$ & $1.934 * * *$ & $(1.451-2.579)$ \\
\hline _cons & 0.421 & $(0.188-0.947)$ & 0.154 & $(0.061-0.391)$ \\
\hline
\end{tabular}


Appendix 4-A: Distribution of cost-related unmet need and other reasons for unmet need for healthcare across the counties (regions)

\begin{tabular}{|c|c|c|c|c|c|c|}
\hline & $A$ & $\mathrm{~B}$ & $\mathrm{D}$ & $E$ & $\mathrm{~F}$ & $\bar{G}$ \\
\hline County & $\begin{array}{l}\text { Cost-related } \\
\text { unmet need } \\
\text { (\%) D/G }\end{array}$ & $\begin{array}{l}\text { Other } \\
\text { reasons for } \\
\text { unmet need } \\
\text { (\%) E/G }\end{array}$ & $\begin{array}{l}\text { Cost- } \\
\text { related } \\
\text { unmet } \\
\text { need }(\mathrm{N})\end{array}$ & $\begin{array}{l}\text { Other } \\
\text { reasons for } \\
\text { unmet } \\
\text { need }(\mathrm{N})\end{array}$ & $\begin{array}{l}\text { Total } \\
\text { Unmet } \\
\text { need } \\
(\mathrm{N})\end{array}$ & $\begin{array}{l}\text { Total } \\
\text { ill/needed } \\
\text { admission } \\
\text { (N) }\end{array}$ \\
\hline Baringo & 0.76 & 3.15 & 6 & 25 & 31 & 794 \\
\hline Bomet & 0.84 & 6.53 & 8 & 62 & 70 & 950 \\
\hline Bungoma & 4.09 & 4.01 & 48 & 47 & 95 & 1,173 \\
\hline Busia & 6.9 & 4.63 & 82 & 55 & 137 & 1,189 \\
\hline Elgeyo Marakwet & 1.36 & 4.07 & 8 & 24 & 32 & 590 \\
\hline Embu & 3.95 & 5.98 & 37 & 56 & 93 & 936 \\
\hline Homabay & 4.15 & 2.15 & 54 & 28 & 82 & 1,300 \\
\hline Isiolo & 4.92 & 1.97 & 35 & 14 & 49 & 712 \\
\hline Kajiado & 0.56 & 3.93 & 4 & 28 & 32 & 712 \\
\hline Kakamega & 3.78 & 1.79 & 57 & 27 & 84 & 1,506 \\
\hline Kericho & 3.17 & 17.92 & 37 & 209 & 246 & 1,166 \\
\hline Kiambu & 1.63 & 3.54 & 12 & 26 & 38 & 734 \\
\hline Kilifi & 2 & 4.95 & 21 & 52 & 73 & 1,050 \\
\hline Kirinyaga & 1.11 & 3.02 & 11 & 30 & 41 & 994 \\
\hline Kisii & 4.26 & 5.60 & 35 & 46 & 81 & 822 \\
\hline Kisumu & 1.6 & 0.80 & 16 & 8 & 24 & 1,002 \\
\hline Kitui & 5.89 & 6.22 & 71 & 75 & 146 & 1,206 \\
\hline Kwale & 3.92 & 2.72 & 39 & 27 & 66 & 994 \\
\hline Laikipia & 3.01 & 3.76 & 20 & 25 & 45 & 665 \\
\hline Lamu & 1.93 & 7.72 & 19 & 76 & 95 & 984 \\
\hline Machakos & 3.27 & 4.73 & 47 & 68 & 115 & 1,438 \\
\hline Makueni & 3.53 & 5.23 & 31 & 46 & 77 & 879 \\
\hline Marsabit & 6.47 & 7.58 & 35 & 41 & 76 & 541 \\
\hline Meru & 1.75 & 4.29 & 18 & 44 & 62 & 1,026 \\
\hline Migori & 2.12 & 0.90 & 33 & 14 & 47 & 1,557 \\
\hline Mombasa & 4.03 & 4.17 & 28 & 29 & 57 & 695 \\
\hline Murang'a & 1.91 & 1.91 & 19 & 19 & 38 & 994 \\
\hline Nairobi & 1.59 & 4.34 & 15 & 41 & 56 & 944 \\
\hline
\end{tabular}




\begin{tabular}{|c|c|c|c|c|c|c|}
\hline Nakuru & 3.7 & 14.95 & 23 & 93 & 116 & 622 \\
\hline Nandi & 4.28 & 0.86 & 35 & 7 & 42 & 818 \\
\hline Narok & 3.03 & 5.52 & 22 & 40 & 62 & 725 \\
\hline Nyamira & 1.95 & 1.39 & 21 & 15 & 36 & 1,078 \\
\hline Nyandarua & 0.94 & 3.24 & 9 & 31 & 40 & 957 \\
\hline Nyeri & 1.16 & 4.25 & 9 & 33 & 42 & 777 \\
\hline Samburu & 4 & 26.71 & 34 & 227 & 261 & 850 \\
\hline Siaya & 1.66 & 3.39 & 20 & 41 & 61 & 1,208 \\
\hline Taita-Taveta & 0.9 & 4.86 & 7 & 38 & 45 & 782 \\
\hline Tanariver & 9.8 & 7.16 & 100 & 73 & 173 & 1,020 \\
\hline Tharaka Nithi & 1.98 & 2.64 & 21 & 28 & 49 & 1,061 \\
\hline Trans Nzoia & 4.21 & 15.23 & 45 & 163 & 208 & 1,070 \\
\hline Turkana & 9.67 & 1.56 & 62 & 10 & 72 & 641 \\
\hline Uasin Gishu & 4.26 & 4.49 & 36 & 38 & 74 & 846 \\
\hline Vihiga & 2.12 & 1.02 & 25 & 12 & 37 & 1,180 \\
\hline West Pokot & 3.15 & 4.86 & 22 & 34 & 56 & 699 \\
\hline Total & & & 1,337 & 2,125 & 3,462 & 41,887 \\
\hline
\end{tabular}


Appendix 4-B: Distribution of the individuals with cost-related unmet need by socioeconomic characteristics

\begin{tabular}{|c|c|c|c|c|c|}
\hline Variables & Categories & $\begin{array}{r}\text { Outpatient } \\
(\%)\end{array}$ & $\begin{array}{r}\text { Inpatient } \\
(\%) \\
\end{array}$ & Total (\%) & $\begin{array}{r}\text { Sample } \\
\mathrm{N}(1,337) \\
\end{array}$ \\
\hline \multicolumn{6}{|l|}{ Predisposing factors } \\
\hline \multirow[t]{3}{*}{ Age group of $\mathrm{HH}$} & Below 25 Years & 2.78 & 2.73 & 2.77 & 37 \\
\hline & $25-40$ Years & 30.99 & 41.41 & 32.98 & 441 \\
\hline & $40+$ Years & 66.23 & 55.86 & 64.25 & 859 \\
\hline \multirow{4}{*}{$\mathrm{HH}$ head education level } & None & 1.85 & 1.98 & 1.88 & 25 \\
\hline & Primary education & 81.37 & 80 & 81.08 & 1,080 \\
\hline & $\begin{array}{l}\text { Secondary } \\
\text { education }\end{array}$ & 14.27 & 13.04 & 14.04 & 187 \\
\hline & Tertiary & 2.5 & 5.14 & 3 & 40 \\
\hline \multirow{2}{*}{ Gender of $\mathrm{HH}$} & Male & 68.36 & 67.19 & 68.14 & 911 \\
\hline & Female & 31.64 & 32.81 & 31.86 & 426 \\
\hline \multirow{2}{*}{$\begin{array}{l}\text { Employment status of } \mathrm{HH} \\
\text { head }\end{array}$} & Unemployed & 16.93 & 19.92 & 17.5 & 234 \\
\hline & Employed & 83.07 & 80 & 82.5 & 1,103 \\
\hline \multicolumn{6}{|l|}{ Need factors } \\
\hline \multirow[t]{2}{*}{ Type of service } & Outpatient & $\mathrm{NA}$ & NA & 80.85 & 1081 \\
\hline & Inpatient & NA & NA & 19.15 & 256 \\
\hline \multirow[t]{3}{*}{ Self-rated health } & Poor & 14.26 & 29.69 & 17.22 & 230 \\
\hline & Satisfactory & 27.41 & 22.66 & 26.5 & 354 \\
\hline & Good & 58.33 & 47.66 & 56.29 & 752 \\
\hline \multirow[t]{2}{*}{ Chronic illness } & No chronic illness & 75.95 & 64.84 & 73.8 & 987 \\
\hline & Chronic illness & 24.05 & 35.16 & 26.18 & 350 \\
\hline \multicolumn{6}{|l|}{ Enabling factors } \\
\hline \multirow[t]{3}{*}{ Household size } & 1-3 Small Size & 26.85 & 22.66 & 26.05 & 348 \\
\hline & 4-6 Medium Size & 43.24 & 38.28 & 42.29 & 565 \\
\hline & 7+ Large Size & 29.91 & 39.06 & 31.66 & 423 \\
\hline \multirow[t]{2}{*}{ Insurance status } & Not insured & 93.43 & 90 & 92.74 & 1,240 \\
\hline & Insured & 6.57 & 10.16 & 7.26 & 97 \\
\hline \multirow[t]{2}{*}{ Residence } & Rural & 70.21 & 70.7 & 70.31 & 940 \\
\hline & Urban & 29.79 & 29.3 & 29.69 & 397 \\
\hline \multirow[t]{5}{*}{ Wealth quintile } & Poorest & 38.61 & 35.94 & 38.1 & 509 \\
\hline & Second & 25.93 & 24.61 & 25.67 & 343 \\
\hline & Middle & 19.63 & 17.97 & 19.31 & 258 \\
\hline & Fourth & 10.74 & 17.97 & 12.13 & 162 \\
\hline & Richest & 5.09 & 3.52 & 4.79 & 64 \\
\hline
\end{tabular}

HH Household; NA Not applicable 
Appendix 4-C. Multilevel and logistic regression models

\begin{tabular}{|c|c|c|c|c|c|}
\hline \multirow[t]{2}{*}{ Factors } & \multirow[t]{2}{*}{ Categories } & \multicolumn{2}{|c|}{ Multilevel Model } & \multicolumn{2}{|c|}{ Logistic regression } \\
\hline & & Odds Ratio & {$[95 \% \mathrm{Cl}]$} & Odds Ratio & {$[95 \% \mathrm{Cl}]$} \\
\hline \multicolumn{6}{|c|}{ Predisposing factors } \\
\hline \multirow{2}{*}{$\begin{array}{l}\text { Gender of the } \\
\text { HH head }\end{array}$} & \multicolumn{5}{|l|}{ Male $[\mathrm{RC}]$} \\
\hline & Female & 1.019 & $(0.897-1.157)$ & 0.968 & $(0.854-1.096)$ \\
\hline \multirow{3}{*}{$\begin{array}{l}\text { Age group of } \\
\text { the HH head }\end{array}$} & \multicolumn{5}{|c|}{ Below 25years [RC] } \\
\hline & $25-40$ years & 1.333 & $(0.936-1.898)$ & 1.198 & $(0.843-1.700)$ \\
\hline & $40+$ years & $1.793 * * *$ & $(1.264-2.545)$ & $1.559 * *$ & $(1.103-2.204)$ \\
\hline \multirow{4}{*}{$\begin{array}{l}\text { Education level } \\
\text { of HH head }\end{array}$} & None $[\mathrm{RC}]$ & & & & \\
\hline & Primary & 0.956 & $(0.624-1.465)$ & 0.736 & $(0.487-1.112)$ \\
\hline & Secondary & 0.669 & $(0.426-1.052)$ & $0.509 * *$ & $(0.329-0.788)$ \\
\hline & Tertiary & $0.534 *$ & $(0.310-0.921)$ & $0.423 * * *$ & $(0.249-0.718)$ \\
\hline \multirow{2}{*}{$\begin{array}{l}\text { Employment } \\
\text { status of } \mathrm{HH} \\
\text { head }\end{array}$} & \multicolumn{5}{|c|}{ Not employed [RC] } \\
\hline & Employed & 0.932 & $(0.786-1.103)$ & $0.725 * * *$ & $(0.623-0.843)$ \\
\hline \multicolumn{6}{|l|}{ Need factors } \\
\hline \multirow[t]{2}{*}{ Type of service } & \multicolumn{5}{|l|}{ Outpatient [RC] } \\
\hline & Inpatient & $1.950 * * *$ & $(1.683-2.260)$ & $1.961 * * *$ & $(1.697-2.265)$ \\
\hline \multirow{3}{*}{$\begin{array}{l}\text { Self-rated } \\
\text { health }\end{array}$} & Poor $[\mathrm{RC}]$ & & & & \\
\hline & Satisfactory & 0.942 & $(0.786-1.129)$ & 0.98 & $(0.821-1.170)$ \\
\hline & Good & $0.567 * * *$ & $(0.477-0.673)$ & $0.598 * * *$ & $(0.505-0.707)$ \\
\hline \multirow[t]{2}{*}{ Chronic IIIness } & No Illness [RC] & & & & \\
\hline & Chronic illness & 1.067 & $(0.924-1.231)$ & 1.077 & $(0.935-1.241)$ \\
\hline \multicolumn{6}{|l|}{ Enabling factors } \\
\hline \multirow[t]{2}{*}{$\begin{array}{l}\text { Insurance } \\
\text { status }\end{array}$} & $\begin{array}{l}\text { Not insured } \\
{[R C]}\end{array}$ & & & & \\
\hline & Insured & $0.505 * * *$ & $(0.401-0.636)$ & $0.463 * * *$ & $(0.369-0.580)$ \\
\hline \multirow[t]{3}{*}{ Household size } & 1-3 Small $[\mathrm{RC}]$ & & & & \\
\hline & $\begin{array}{l}\text { 4-6 Medium } \\
\text { Size }\end{array}$ & $0.693 * * *$ & $(0.600-0.802)$ & $0.732 * * \star$ & $(0.635-0.845)$ \\
\hline & 7+ Large Size & $0.717 * * *$ & $(0.611-0.842)$ & $0.816 * *$ & $(0.698-0.953)$ \\
\hline \multirow[t]{2}{*}{ Residence } & Rural $[\mathrm{RC}]$ & & & & \\
\hline & Urban & $1.259 * * *$ & $(1.094-1.449)$ & $1.274 * * *$ & $(1.116-1.454)$ \\
\hline
\end{tabular}




\begin{tabular}{|c|c|c|c|c|c|}
\hline \multirow[t]{6}{*}{ Wealth Index } & \multicolumn{5}{|l|}{ Poorest [RC] } \\
\hline & Second quintile & $0.845 *$ & $(0.724-0.987)$ & $0.735 * * *$ & $(0.638-0.848)$ \\
\hline & Middle quintile & $0.632 * * *$ & $(0.532-0.751)$ & $0.585 * * *$ & $(0.499-0.686)$ \\
\hline & Fourth quintile & $0.470 * * *$ & $(0.380-0.579)$ & $0.440 * * *$ & $(0.361-0.535)$ \\
\hline & Richest quintile & $0.314 * * *$ & $(0.226-0.436)$ & $0.324 * * *$ & $(0.240-0.439)$ \\
\hline & _cons & $0.055 * * *$ & $(0.030-0.103)$ & $0.112 * * *$ & (0.064-0.198) \\
\hline \multirow[t]{7}{*}{ Random effects } & $\begin{array}{l}\text { /Insig2u } \\
\text { [County } \\
\text { variance] }\end{array}$ & -1.074 & $(-1.573--0.574)$ & & \\
\hline & $\begin{array}{l}\text { sigma_u } \\
\text { [Residual } \\
\text { variance] }\end{array}$ & $\begin{array}{l}0.585 \\
{[0.074]}\end{array}$ & $(0.455-0.750)$ & & \\
\hline & $\begin{array}{l}\text { Rho [Intraclass } \\
\text { correlation } \\
(\mathrm{ICC})]\end{array}$ & $\begin{array}{l}0.094 \\
{[0.022]}\end{array}$ & $(0.059-0.146)$ & & \\
\hline & Log likelihood & -5426.35 & & -5554.9141 & \\
\hline & Wald chi2(19) & 515.03 & & 668.35 & \\
\hline & $\begin{array}{l}\text { Number of } \\
\text { observations }\end{array}$ & 41,646 & & 41,646 & \\
\hline & $\begin{array}{l}\text { Number of } \\
\text { groups }\end{array}$ & 44 & & & \\
\hline
\end{tabular}

Log likelihood ratio test: logistic Vs multilevel regression: chi2(2) $=257.137$, Prob $<0.001$

$P<0.05^{\star}, 0.01^{\star *}$ and $0.001^{\star * *}$, Ref Reference, HH Household 
Appendix 5-A Description of the sample by type of shock and time of shock

\begin{tabular}{|c|c|c|c|}
\hline \multicolumn{2}{|c|}{ Type of shock and duration } & \multirow{2}{*}{$\begin{array}{r}\text { Percent } \\
30.82 \%\end{array}$} & \multirow{2}{*}{$\frac{\text { Frequency }(\mathrm{N})}{5023}$} \\
\hline General shocks & No shocks & & \\
\hline & All shocks & $69.18 \%$ & 11274 \\
\hline & Multiple shocks & $44.92 \%$ & 7320 \\
\hline & One shock & $24.26 \%$ & 3954 \\
\hline \multirow[t]{3}{*}{ One shock } & Income shocks & $45.02 \%$ & 1780 \\
\hline & Asset shocks & $13.91 \%$ & 550 \\
\hline & Income \& asset shocks & $27.14 \%$ & 1073 \\
\hline \multirow{3}{*}{$\begin{array}{l}\text { Shocks in 7-12 } \\
\text { months }\end{array}$} & Income shocks in the last $7-12$ months & $6.45 \%$ & 255 \\
\hline & Asset shocks in the last $7-12$ months & $2.33 \%$ & 92 \\
\hline & Income $\&$ asset shocks in the last $7-12$ months & $4.45 \%$ & 176 \\
\hline \multirow[t]{3}{*}{ Shocks in 1-6 months } & Income shocks in the last 1-6 months & $23.42 \%$ & 926 \\
\hline & Asset shocks in the last $1-6$ months & $7.64 \%$ & 302 \\
\hline & Income $\&$ asset shocks in the last 1-6 months & $15.30 \%$ & 605 \\
\hline \multirow{3}{*}{$\begin{array}{l}\text { Shocks in below I } \\
\text { month }\end{array}$} & Income shocks in below 1 Month & $15.15 \%$ & 599 \\
\hline & Asset shocks in below 1 Month & $3.95 \%$ & 156 \\
\hline & Income $\&$ asset shocks in below 1 Month & $7.38 \%$ & 292 \\
\hline
\end{tabular}


Appendix 5-B Mantel Hanzel test analysis results All shocks

\begin{tabular}{|c|c|c|c|c|}
\hline \multirow[t]{2}{*}{ Gamma(Г) } & \multicolumn{2}{|l|}{ Test statistic } & \multicolumn{2}{|l|}{ Significance level } \\
\hline & $\begin{array}{l}\text { Overestimation } \\
(\text { Q_mh+) }\end{array}$ & $\begin{array}{l}\text { Underestimation } \\
\text { (Q_mh-) }\end{array}$ & $\begin{array}{l}\text { Overestimation } \\
(\text { p_mh }+)\end{array}$ & $\begin{array}{l}\text { Overestimation } \\
(\mathrm{Q} \text { mh+) }\end{array}$ \\
\hline 1.0 & 2.001 & 2.001 & 0.023 & 0.023 \\
\hline 1.1 & 4.134 & .0834 & 0.000 & 0.466 \\
\hline 1.2 & 6.085 & 2.027 & 0.000 & 0.021 \\
\hline 1.3 & 7.887 & 3.817 & 0.000 & 0.000 \\
\hline 1.4 & 9.561 & 5.479 & 0.000 & 0.000 \\
\hline 1.5 & 11.126 & 7.030 & 0.000 & 0.000 \\
\hline 1.6 & 12.597 & 8.486 & 0.000 & 0.000 \\
\hline 1.7 & 13.985 & 9.859 & 0.000 & 0.000 \\
\hline 1.8 & 15.300 & 11.161 & 0.000 & 0.000 \\
\hline 1.9 & 16.550 & 12.397 & 0.000 & 0.000 \\
\hline 2.0 & 17.741 & 13.577 & 0.000 & 0.000 \\
\hline
\end{tabular}

\section{Multiple shocks}

\begin{tabular}{|c|c|c|c|c|}
\hline \multirow[t]{2}{*}{ Gamma(Г) } & \multicolumn{2}{|l|}{ Test statistic } & \multicolumn{2}{|l|}{ Significance level } \\
\hline & $\begin{array}{l}\text { Overestimation } \\
(\text { Q_mh+) }\end{array}$ & $\begin{array}{l}\text { Underestimation } \\
\text { (Q_mh-) }\end{array}$ & $\begin{array}{l}\text { Overestimation } \\
(\text { p_mh }+)\end{array}$ & $\begin{array}{l}\text { Overestimation } \\
(\mathrm{Q} \text { mh+) }\end{array}$ \\
\hline 1.0 & 2.325 & 2.325 & 0.010 & 0.010 \\
\hline 1.1 & 4.303 & 0.350 & 0.000 & 0.362 \\
\hline 1.2 & 6.113 & 1.403 & 0.000 & 0.080 \\
\hline 1.3 & 7.784 & 3.062 & 0.000 & 0.001 \\
\hline 1.4 & 9.337 & 4.600 & 0.000 & 0.000 \\
\hline 1.5 & 10.789 & 6.036 & 0.000 & 0.000 \\
\hline 1.6 & 12.154 & 7.384 & 0.000 & 0.000 \\
\hline 1.7 & 13.442 & 8.653 & 0.000 & 0.000 \\
\hline 1.8 & 14.663 & 9.855 & 0.000 & 0.000 \\
\hline 1.9 & 15.823 & 10.997 & 0.000 & 0.000 \\
\hline 2.0 & 16.930 & 12.085 & 0.000 & 0.000 \\
\hline
\end{tabular}




\section{One shock}

\begin{tabular}{|c|c|c|c|c|}
\hline \multirow[t]{2}{*}{ Gamma(Г) } & \multicolumn{2}{|l|}{ Test statistic } & \multicolumn{2}{|l|}{ Significance level } \\
\hline & $\begin{array}{l}\text { Overestimation } \\
(\text { Q_mh+) }\end{array}$ & $\begin{array}{l}\text { Underestimation } \\
\text { (Q_mh-) }\end{array}$ & $\begin{array}{l}\text { Overestimation } \\
\left(p \_m h+\right)\end{array}$ & $\begin{array}{l}\text { Underestimation } \\
\text { (p_mh-) }\end{array}$ \\
\hline 1.0 & 0.604 & 0.604 & 0.272 & 0.272 \\
\hline 1.1 & 2.299 & 1.033 & 0.010 & 0.150 \\
\hline 1.2 & 3.849 & 2.581 & 0.000 & 0.004 \\
\hline 1.3 & 5.279 & 4.007 & 0.000 & 0.000 \\
\hline 1.4 & 6.606 & 5.331 & 0.000 & 0.000 \\
\hline 1.5 & 7.848 & 6.566 & 0.000 & 0.000 \\
\hline 1.6 & 9.014 & 7.727 & 0.000 & 0.000 \\
\hline 1.7 & 10.115 & 8.812 & 0.000 & 0.000 \\
\hline 1.8 & 11.158 & 9.856 & 0.000 & 0.000 \\
\hline 1.9 & 12.150 & 10.840 & 0.000 & 0.000 \\
\hline 2.0 & 13.096 & 11.777 & 0.000 & 0.000 \\
\hline
\end{tabular}


Appendix 5-C. tebalance Analysis Results

All Shocks_ Covariate balance summary

\begin{tabular}{|c|c|c|c|c|}
\hline & & Raw & Matched & \\
\hline Number of observations & & 16,290 & 22,540 & \\
\hline Treated observations $=$ & & 11,270 & 11,270 & \\
\hline Control observations $=$ & & 5,020 & 11,270 & \\
\hline & Standard & rences & Variance & \\
\hline & Raw & Matched & Raw & Matched \\
\hline $\begin{array}{l}\text { Gender of household hea } \\
\text { Male }\end{array}$ & & & & \\
\hline Female & 0.077 & 0.006 & 1.053 & 1.004 \\
\hline $\begin{array}{l}\text { Age group of household } h \\
\text { Below } 25 \text { years }\end{array}$ & & & & \\
\hline $25-40$ years & -0.078 & -0.002 & 1.004 & 1.000 \\
\hline $40+$ years & 0.113 & -0.001 & 1.027 & 1.000 \\
\hline $\begin{array}{l}\text { Education level of houser } \\
\text { Primary level }\end{array}$ & old head & & & \\
\hline Secondary level & -0.048 & 0.003 & 0.939 & 1.004 \\
\hline Tertiary level & -0.115 & 0.008 & 0.734 & 1.026 \\
\hline Others & 0.021 & 0.003 & 1.035 & 1.004 \\
\hline Household Size & & & & \\
\hline 1-3 Small Size & & & & \\
\hline 4-6 Medium Size & -0.013 & 0.001 & 0.998 & 1.000 \\
\hline 7+ Large Size & 0.200 & 0.003 & 1.265 & 1.003 \\
\hline $\begin{array}{l}\text { Residence } \\
\text { Rural }\end{array}$ & & & & \\
\hline Urban & -0.199 & 0.002 & 0.895 & 1.001 \\
\hline $\begin{array}{l}\text { Wealth status } \\
\text { Better off }\end{array}$ & & & & \\
\hline $\begin{array}{l}\text { Worse off } \\
\text { Insurance status } \\
\text { Insured }\end{array}$ & 0.058 & 0.003 & 1.038 & 1.002 \\
\hline Not insured & 0.085 & -0.006 & 0.869 & 1.012 \\
\hline
\end{tabular}


Multiple Shocks_ Covariate balance summary

\begin{tabular}{|c|c|c|c|c|}
\hline \multicolumn{5}{|c|}{ Raw Matched } \\
\hline Number of observations $=$ & 12,338 & \multicolumn{3}{|l|}{14,636} \\
\hline Treated observations $=$ & 7,318 & \multicolumn{3}{|l|}{7,318} \\
\hline \multirow[t]{3}{*}{ Control observations $=$} & 5,020 & \multicolumn{3}{|l|}{7,318} \\
\hline & \multicolumn{2}{|c|}{ Standardized differences } & \multicolumn{2}{|c|}{ Variance ratio } \\
\hline & Raw & Matched & Raw & Matched \\
\hline \multicolumn{5}{|c|}{ Gender of household head } \\
\hline Female & 0.094 & 0.001 & 1.063 & 1.000 \\
\hline \multicolumn{5}{|c|}{ Age group of household head } \\
\hline $25-40$ years & -0.089 & -0.003 & 1.004 & 1.000 \\
\hline $40+$ years & 0.134 & 0.001 & 1.030 & 1.000 \\
\hline \multicolumn{5}{|c|}{ Education level of household head } \\
\hline Secondary level & -0.030 & -0.001 & 0.962 & 0.998 \\
\hline Tertiary level & -0.121 & 0.011 & 0.721 & 1.033 \\
\hline Others & 0.017 & -0.001 & 1.028 & 0.998 \\
\hline \multicolumn{5}{|l|}{ Household Size } \\
\hline 4-6 Medium Size & 0.019 & -0.002 & 1.002 & 1.000 \\
\hline 7+ Large Size & 0.228 & 0.004 & 1.297 & 1.004 \\
\hline \multicolumn{5}{|l|}{ Residence } \\
\hline Urban & -0.260 & 0.001 & 0.850 & 1.001 \\
\hline \multicolumn{5}{|l|}{ Wealth status } \\
\hline $\begin{array}{l}\text { Worse off } \\
\text { Insurance status } \\
\text { Insured }\end{array}$ & 0.060 & 0.008 & 1.039 & 1.005 \\
\hline Not insured & 0.102 & -0.006 & 0.841 & 1.011 \\
\hline
\end{tabular}


One Shock_ Covariate balance summary

\begin{tabular}{|c|c|c|c|c|}
\hline & Raw & Matched & & \\
\hline Number of observations $=$ & 8,972 & 7,904 & & \\
\hline Treated observations $=$ & 3,952 & 3,952 & & \\
\hline \multirow[t]{3}{*}{ Control observations $=$} & 5,020 & 3,952 & & \\
\hline & \multicolumn{2}{|c|}{ Standardized differences } & \multicolumn{2}{|c|}{ Variance ratio } \\
\hline & Raw & Matched & Raw & Matched \\
\hline \multicolumn{5}{|l|}{$\begin{array}{l}\text { Gender of household head } \\
\text { Male }\end{array}$} \\
\hline Female & 0.047 & 0.001 & 1.034 & 1.001 \\
\hline \multicolumn{5}{|l|}{$\begin{array}{l}\text { Age group of household head } \\
\text { Below } 25 \text { years }\end{array}$} \\
\hline $25-40$ years & -0.056 & -0.010 & 1.004 & 1.000 \\
\hline $40+$ years & 0.073 & 0.001 & 1.021 & 1.000 \\
\hline \multicolumn{5}{|c|}{ Education level of household head } \\
\hline Secondary level & -0.081 & -0.004 & 0.896 & 0.994 \\
\hline Tertiary level & -0.105 & 0.005 & 0.759 & 1.013 \\
\hline Others & 0.029 & 0.006 & 1.047 & 1.010 \\
\hline \multicolumn{5}{|l|}{ Household Size } \\
\hline \multicolumn{5}{|l|}{ 1-3 Small Size } \\
\hline 4-6 Medium Size & -0.071 & -0.002 & 0.985 & 0.999 \\
\hline 7+ Large Size & 0.146 & 0.004 & 1.201 & 1.004 \\
\hline \multicolumn{5}{|l|}{ Residence } \\
\hline \multicolumn{5}{|l|}{ Rural } \\
\hline Urban & -0.091 & 0.005 & 0.961 & 1.003 \\
\hline \multicolumn{4}{|l|}{ Wealth status } & \\
\hline $\begin{array}{l}\text { Worse off } \\
\text { Insurance status } \\
\text { Insured }\end{array}$ & 0.055 & -0.006 & 1.036 & 0.996 \\
\hline Not insured & 0.053 & -0.005 & 0.918 & 1.009 \\
\hline
\end{tabular}


Income Shocks _ Covariate balance summary

$\begin{array}{lcc} & \text { Raw } & \text { Matched } \\ \text { Number of observations }= & 6,799 & 3,558 \\ \text { Treated observations }= & 1,779 & 1,779 \\ \text { Control observations }= & 5,020 & 1,779\end{array}$

\begin{tabular}{|c|c|c|c|c|}
\hline & \multicolumn{2}{|c|}{ Standardized differences } & \multicolumn{2}{|c|}{ Variance ratio } \\
\hline & Raw & Matched & Raw & Matched \\
\hline \multicolumn{5}{|c|}{ Gender of household head } \\
\hline Male & 0.004 & -0.008 & 1.003 & 0.994 \\
\hline \multicolumn{5}{|l|}{ Female } \\
\hline \multicolumn{5}{|c|}{ Age group of household head } \\
\hline \multicolumn{5}{|l|}{ Below 25 years } \\
\hline $25-40$ years & -0.070 & -0.000 & 1.005 & 1 \\
\hline $40+$ years & 0.079 & 0.001 & 1.022 & 1.000 \\
\hline \multicolumn{5}{|c|}{ Education level of household head } \\
\hline \multicolumn{5}{|l|}{ Primary level } \\
\hline Secondary level & -0.014 & -0.003 & 0.983 & 0.997 \\
\hline Tertiary level & -0.086 & 0.002 & 0.801 & 1.006 \\
\hline Others & -0.061 & 0.003 & 0.899 & 1.006 \\
\hline \multicolumn{5}{|l|}{ Household Size } \\
\hline \multicolumn{5}{|l|}{ 1-3 Small Size } \\
\hline 4-6 Medium Size & -0.016 & 0.002 & 0.998 & 1.000 \\
\hline 7+ Large Size & 0.075 & 0.003 & 1.107 & 1.003 \\
\hline \multicolumn{5}{|l|}{ Residence } \\
\hline \multicolumn{5}{|l|}{ Rural } \\
\hline Urban & -0.038 & -0.003 & 0.986 & 0.999 \\
\hline \multicolumn{5}{|l|}{ Wealth status } \\
\hline $\begin{array}{l}\text { Worse off } \\
\text { Insurance status } \\
\text { Insured }\end{array}$ & -0.044 & 0.002 & 0.967 & 1.002 \\
\hline Not insured & -0.040 & -0.004 & 1.060 & 1.006 \\
\hline
\end{tabular}


Asset Shocks _ Covariate balance summary

$\begin{array}{lrc} & \text { Raw } & \text { Matched } \\ \text { Number of observations }= & 5,570 & 1,100 \\ \text { Treated observations }= & 550 & 550 \\ \text { Control observations }= & 5,020 & 550\end{array}$

\begin{tabular}{|c|c|c|c|c|}
\hline & \multicolumn{2}{|c|}{ Standardized differences } & \multicolumn{2}{|c|}{ Variance ratio } \\
\hline & Raw & Matched & Raw & Matched \\
\hline \multicolumn{5}{|c|}{ Gender of household head } \\
\hline \multicolumn{5}{|l|}{ Male } \\
\hline Female & 0.027 & -0.004 & 1.022 & 0.997 \\
\hline \multicolumn{5}{|c|}{ Age group of household head } \\
\hline \multicolumn{5}{|l|}{ Below 25 years } \\
\hline $25-40$ years & -0.053 & 0.004 & 1.006 & 1.000 \\
\hline $40+$ years & 0.111 & -0.007 & 1.029 & 0.999 \\
\hline \multicolumn{5}{|c|}{ Education level of household head } \\
\hline \multicolumn{5}{|l|}{ Primary level } \\
\hline Secondary level & -0.169 & -0.014 & 0.779 & 0.975 \\
\hline Tertiary level & -0.084 & 0.006 & 0.807 & 1.018 \\
\hline Others & 0.083 & 0.004 & 1.135 & 1.006 \\
\hline \multicolumn{5}{|l|}{ Household Size } \\
\hline \multicolumn{5}{|l|}{ 1-3 Small Size } \\
\hline 4-6 Medium Size & -0.203 & 0.000 & 0.934 & 1.000 \\
\hline 7+ Large Size & 0.307 & 0.000 & 1.379 & 1.000 \\
\hline \multicolumn{5}{|l|}{ Residence } \\
\hline \multicolumn{5}{|l|}{ Rural } \\
\hline Urban & -0.165 & 0.000 & 0.919 & 1.000 \\
\hline \multicolumn{5}{|l|}{ Wealth status } \\
\hline \multicolumn{5}{|l|}{ Better off } \\
\hline Worse off & 0.121 & -0.004 & 1.073 & 0.998 \\
\hline \multicolumn{5}{|l|}{ Insurance status } \\
\hline Insured & & & & \\
\hline Not insured & 0.117 & 0.000 & 0.819 & 1.000 \\
\hline
\end{tabular}


Both Income \& Asset Shocks _ Covariate balance summary

$\begin{array}{llc} & \text { Raw } & \text { Matched } \\ \text { Number of observations }= & 6,092 & 2,144 \\ \text { Treated observations }= & 1,072 & 1,072 \\ \text { Control observations }= & 5,020 & 1,072\end{array}$

\begin{tabular}{|c|c|c|c|c|}
\hline & \multicolumn{2}{|c|}{ Standardized differences } & \multicolumn{2}{|c|}{ Variance ratio } \\
\hline & Raw & Matched & Raw & Matched \\
\hline \multicolumn{5}{|c|}{ Gender of household head } \\
\hline \multicolumn{5}{|l|}{ Male } \\
\hline Female & 0.094 & 0.002 & 1.065 & 1.001 \\
\hline \multicolumn{5}{|c|}{ Age group of household head } \\
\hline \multicolumn{5}{|c|}{ Below 25 years } \\
\hline $25-40$ years & -0.097 & -0.015 & 1.004 & 0.999 \\
\hline $40+$ years & 0.133 & 0.009 & 1.031 & 1.001 \\
\hline \multicolumn{5}{|c|}{ Education level of household head } \\
\hline Secondary level & -0.157 & -0.005 & 0.794 & 0.992 \\
\hline Tertiary level & -0.135 & 0.000 & 0.692 & 1.000 \\
\hline Others & 0.175 & 0.011 & 1.272 & 1.012 \\
\hline \multicolumn{5}{|l|}{ Household Size } \\
\hline \multicolumn{5}{|l|}{ 1-3 Small Size } \\
\hline 4-6 Medium Size & -0.118 & -0.006 & 0.971 & 0.998 \\
\hline 7+ Large Size & 0.231 & 0.000 & 1.302 & 1.000 \\
\hline \multicolumn{5}{|l|}{ Residence } \\
\hline \multicolumn{5}{|l|}{ Rural } \\
\hline Urban & -0.291 & 0.002 & 0.826 & 1.002 \\
\hline \multicolumn{5}{|l|}{ Wealth status } \\
\hline \multicolumn{5}{|l|}{ Better off } \\
\hline Worse off & 0.116 & 0.004 & 1.069 & 1.002 \\
\hline \multicolumn{5}{|l|}{ Insurance status } \\
\hline Insured & & & & \\
\hline Not insured & 0.208 & -0.006 & 0.674 & 1.013 \\
\hline
\end{tabular}


Appendix 5-D: Density plots for all shocks, multiple shocks and one shock
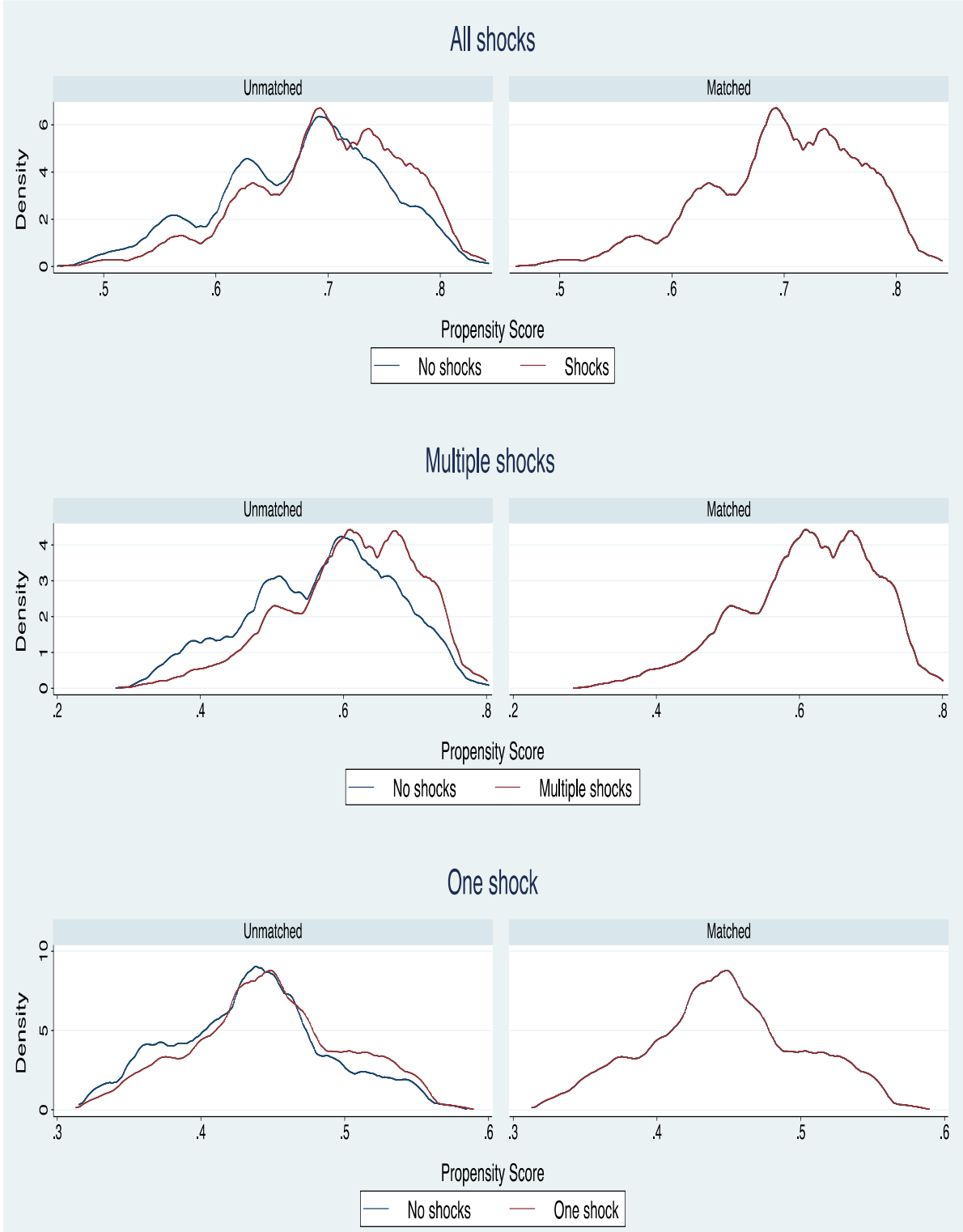


\section{Summary}

This dissertation is underpinned by the global call to achieve universal health coverage (UHC), including financial risk protection and access to quality healthcare services. Kenya has embraced this commitment in its 2010 constitution, by promising to ensure the right to health for the entire population. The Kenya Health Policy 2014 2030 is grounded on the principle of protection of human rights through a focus on equity of access. In addition, UHC is among "the big four" priority areas under the current presidency, which demonstrates the government's commitment and political goodwill to achieving better health for all.

Historically, the Kenyan health system was centralised at the national level, a system that was criticised for its regional discrepancies in healthcare service distribution, disparities in resource allocations and inequitable access to quality healthcare services. These disparities partly informed the "spirit" of devolution as per the constitution of 2010 , to among other objectives ensure a more equitable sharing of national and local resources, thus reducing inequities. In 2013 the healthcare system was decentralised to the new governance unit(counties) as part of implementing the constitution. The national level is now responsible for policy formulation, and national referral services, while the county governments are responsible for the implementation of the policy direction and service delivery.

Inequities in access to healthcare services continue to persist in Kenya, with more than one million people pushed into poverty annually due to healthcare payment. Also, only a fifth of the 47 million population has some form of health insurance. Many have limited access to healthcare services due to cost-related barriers, whereas unanticipated risks like economic shocks limit others, even those with the ability to pay for care. While various measures have been used to assess equity of access, there are limitations in using only one measure given that each measure defines a specific perspective. For instance, catastrophic health expenditure (CHE) commonly used to gauge financial risk protection only assesses people who have used healthcare services, but not those who forgo them.

This dissertation, therefore, extends beyond the general measurement of financial risk protection to explore other perspectives of inequity in access. It assesses the extent of socioeconomic inequalities in financial risk protection, explores the costrelated barriers to access healthcare services considering variations across and within the regions (counties), and the influence of shocks on the ability of households to invest in healthcare.

The dissertation utilises two nationally representative datasets to perform these analyses. First, the Kenya Household and Health Utilisation Survey (KHHEUS) data (2007 and 2013) is used to analyse the socioeconomic inequalities in catastrophic health expenditure and the cost-related unmet need for healthcare services. The KHHEUS is a cross-sectional survey with data collected by the Ministry of Health and the Kenya 
National Bureau of Statistics (KNBS). Second, to analyse household shocks, the dissertation utilises data from the 2015/2016 Kenya Integrated Household Budget Survey (KIHBS), which is implemented by the KNBS under the Kenya Statistics Programme for Results (KSPforR) project.

To respond to the main aim, this dissertation answers the following four key questions:

1. What is the extent, variation and the underlying determinants of CHE across Sub-Saharan Africa (SSA) countries?

2. What is the extent of socioeconomic inequalities in $\mathrm{CHE}$, the associated determinants and the changes over time in Kenya?

3. Do sub-region variations influence the unmet need for healthcare services due to cost barriers?

4. What is the effect of household shocks on the utilisation of healthcare services in Kenya?

Overall, the dissertation is organised into an introductory chapter, four main chapters that comprehensively address the four questions and a concluding chapter that summarises the findings and provides implications for future policy and research. The chapters are summarised as follows:

Chapter 1 introduces the dissertation and provides a background on the areas of interest, based on the existing literature. Further, this chapter presents the rationale of the research considering global, regional and country-level priorities. The context and history of the healthcare system and financing in Kenya, which forms the basis for this study, is also discussed in this chapter. The main aim of the research and the research questions are also described, including the methodological approaches applied to respond to each of the questions.

Chapter 2 of the dissertation seeks to provide a broader regional outlook on financial risk protection in SSA from which Kenya can benchmark, gauge its performance and draw lessons from other countries' experiences. It aims, therefore, to explore the extent of CHE across the various SSA countries and the underlying determinants of CHE, including the reported impoverishment rates due to healthcare payments. To address this aim, the chapter applies a systematic scoping review approach guided by the Preferred Reporting Items for Systematic Reviews and Meta-Analyses (PRISMA) tool that provides key items considered essential for systematic review and meta-analysis reporting. The review searched for articles indexed in various databases including PubMed, EBSCO (EconLit, PsycINFO, CINAHL), Web of Science and JSTOR. In addition, grey literature from $\mathrm{WHO}$ and the World Bank virtual libraries was identified to complement the database searches. The outcome of interest was the incidence of catastrophic health expenditure, while the secondary outcomes were the determinants and the proportion of households impoverished. To ensure the robustness of the studies included in the review, the quality assessment tool for observational cohort and cross- 
sectional studies was applied to evaluate the quality. Finally, 34 studies from across 17 SSA countries met the inclusion criteria and the quality assessment for the full review.

Overall, the analyses showed a higher CHE incidence among countries in the West African region in comparison to other regions. However, this could be due to the use of convenient sampling of pre-selected population groups, unlike nationally representative samples used in other countries. Type of illness and household head characteristics such as age, gender, age group, education level and employment status were seen as key determinants of CHE. Other determinants of CHE included households' characteristics such as health insurance status, size, economic status, rural-urban location, presence of elderly and under-five children and type of health provider where care was sought. Interestingly, formal health insurance status was associated with both lower odds and higher odds of experiencing $\mathrm{CHE}$, while in some studies, insurance was not a significant determinant of $\mathrm{CHE}$. This was mainly due to the relatively low uptake of health insurance across SSA countries. However, informal networks and mutual organisations were seen as key determinants in reducing the incidence of $\mathrm{CHE}$, emphasising the significant role of informal networks in cushioning financial hardship.

These results underscore the pervasiveness and rising incidence of $\mathrm{CHE}$ in many SSA countries over time. Impoverishment was also seen to vary widely across the countries. In accordance with the findings from this chapter, the main recommendation was to intensify insurance uptake to reach the underserved, but more importantly, to leverage and draw innovations from mutual or informal networks to increase insurance coverage. In addition, there is a need to draw synergies and efficiencies in financing across disease areas as some illnesses were associated with higher costs than others.

Although both the rich and poor were observed to experience catastrophic costs, poor households were most at risk. This points to the existence of disparities in $\mathrm{CHE}$ among these socioeconomic groups. However, the majority of the studies reviewed did not assess the inequalities, and even the few that did failed to examine the drivers of inequalities in CHE and, more importantly, the changes over time. In light of this, Chapter 3 responds to the second question regarding the extent of socioeconomic inequalities in $\mathrm{CHE}$, the associated determinants and the changes over time in Kenya. Using KHHEUS data from 2007 and 2013, this chapter assesses the inequalities in CHE across the socioeconomic groups and the drivers that sustain these inequalities. The concentration index is used to measure the socioeconomic inequality in CHE. Households are classified into socioeconomic quintiles using per capita consumption expenditure. The concentration index of $\mathrm{CHE}$ is then decomposed into its determinants to assess the relative contribution of its covariates. Finally, an Oaxaca-type decomposition of the change in a concentration index is applied to assess the changes in CHE inequalities over time.

The findings show that the incidence of CHE has declined over the years, with the rich experiencing a relatively higher decline than the poor. However, the inequality in CHE has increased over time and is disproportionately concentrated amongst the less 
well-off. Household head employment status and wealth status of the household were seen as the main contributors to inequalities in CHE. Furthermore, insurance status of the household was seen to have a minimal significant effect, perhaps due to the small proportion of households that were insured, coupled with a limited insurance package. However, this points to the critical role of insurance in cushioning populations against the financial burden of ill health.

The changes in the elasticities and inequalities of the socioeconomic determinants explained the changes in the inequalities over time. Elasticities in the household economic status was the main contributor to the change (increase) in $\mathrm{CHE}$ inequality over time. These findings emphasise the existence of socioeconomic inequalities that disadvantage the poor in Kenya, suggesting that the mechanisms in place offer inadequate financial protection for the poor and vulnerable. Findings from this chapter lead to recommendations for a crucial multisectoral approach to address existing socioeconomic inequalities and hence, accelerate the achievement of equity in access to healthcare services.

While CHE is a robust measure of financial risk protection, it is based on out-ofpocket payments; hence, it only assesses those individuals who seek healthcare services and not those who refrain from seeking care. Therefore, it is a limited measure for the overall healthcare protection of the entire population. For this reason, and to understand the financial protection for the wider population, it is important to consider those who refrain from seeking care. In this regard, Chapter 4 assesses the cost-related unmet need for healthcare services confounding for variations across the country's regions (counties). Kenya decentralised the healthcare system to the counties, that are now charged with the direct implementation of the policy directives and service delivery. However, these counties vary by socioeconomic profiles and health indicators. Therefore, assessing the regional variations in the unmet need for care can inform the prioritisation of healthcare services in counties that are lagging behind.

Using KHHEUS 2013 data, this dissertation assessed the cost-related unmet need for healthcare services. Self-reported unmet need due to lack of money and high costs of care was used to compute the outcome of interest. A multilevel regression model was applied to explore the factors associated with the cost-related unmet need, factoring the variations across the counties. The results indicate that variations exist across counties with the unmet need being relatively high among inpatient services as compared to outpatient services. The difference at the county level contributed to the variations in the cost-related unmet need for healthcare services. Factors that contribute to higher odds of the cost-related unmet need included older household members, urban residence, inpatient services and lower wealth quintiles. In contrast, factors that contributed to reducing the odds of the cost-related unmet need included education level of household head, good self-rated health, larger household size, insured households and higher wealth quintiles. 
The multilevel model significantly improved the measure for the cost-related unmet need controlling for the effect of county-level variations. The differences at the county level are seen to significantly contribute to the varying rates of the cost-related unmet need. This points to the differences in socioeconomic profiles in the counties that predispose households to varying health-seeking behaviours. Therefore, a multi-layered approach is paramount to addressing variations in the unmet need, as it helps to contextualise and prioritise policy factors or decisions that are relevant at the individual, community or regional level as well as the national level.

Finally, this chapter underscores that the ability to pay is key to enabling access to healthcare services. However, the household's ability to invest in healthcare services could be limited by unanticipated risks. Thus, Chapter 5, explores the impact of households' shocks on the utilisation of healthcare services. This is important to understand as financial setbacks can disrupt the household's economic status, even those with relatively high incomes. Financial shocks due to unforeseen expenses and income losses may cause immediate strain making it more challenging to build or rebuild a financial cushion.

The study utilises the KIHBS 2015/2016, which is the first survey in Kenya to collect comprehensive data on shocks from the households. It therefore examines the impact of household shocks on the utilisation of healthcare services. A propensity score matching approach is used to construct a quasi-experimental design adjusting for selection bias of households that experienced shocks.

The results indicate that overall, shocks reduce the probability of utilising healthcare services when households are confronted with an illness. Multiple shocks in a household were seen to exacerbate the risk of not seeking healthcare services when needed, with a higher negative effect on the utilisation of healthcare services compared to households that experienced one shock. Overall, asset shocks reduced the probability of seeking healthcare services, while due to the smoothing out of income shocks through the sale of assets and borrowing, income shocks had no significant influence on the utilisation of healthcare services. The negative effect of asset shocks increased as the time to illness reduced. This is because a more profound effect on a financial constrained household's decision to seek care is expected for shocks that may occur closer to the time of the illness. Conversely, assets are often used to cushion economic shocks by either selling or using them as collateral for borrowing more assets.

The findings underscore that shocks may limit the capacity of households to afford and access healthcare services when needed. This calls for broadening of social protection programs to integrate mechanisms that can enable households to build resilience to shocks, while cushioning them from financial hardships. Also, there is a need to expedite the expansion of other measures that offer financial protection to households when seeking healthcare services, such as health insurance schemes. This would ultimately cushion households from forgoing care due to costs, especially the poor and vulnerable ones, even when confronted with unanticipated risks. 
Finally, Chapter 6 summarises the cross-cutting discussions and conclusions from all of the chapters into five key statements, including implications for future policy and research. The main findings are summarised as follows:

- $\quad$ Statement 1: The poor are the most vulnerable to catastrophic payments, yet they are least protected from the financial burden imposed by healthcare costs.

A consistent finding across the chapters of this dissertation was that the poor are most burdened by healthcare costs given that even a small amount of payment could turn catastrophic. A significant number of individuals, particularly the poor, do not access care due to cost-related barriers. This means that the existing risk protection mechanisms have not been sufficient to provide a cushion to economically vulnerable groups. For instance, the coverage by the National Hospital Insurance Fund(NHIF) is based on a premium, which is still unaffordable to the poor. Furthermore, the results show that the inequalities in catastrophic payments between the poor and well-off have increased over time. This points to the need to further understand and address the drivers of socioeconomic inequalities to improve pro-poor policy interventions. These results underscore the vital role of financial risk protection in gaining entry into the healthcare system and therefore, influence equity of access to healthcare services.

- $\quad$ Statement 2: Disparities in financial risk protection allude to the existence of inequities within various levels of the healthcare delivery system.

This dissertation not only observed disparities in financial risk protection across various groups, but also various regions. These disparities are a reflection of the significant inequities that exist within the healthcare delivery system. Furthermore, Kenya has witnessed an upsurge of private healthcare services, which are prohibitive in costs as many wealthier households seek better quality care. This emphasises that access to healthcare services is influenced by the interplay between system-level and individual/household-level factors. Evidence shows that these system-level inequities may include a shortage of healthcare workers, inadequate healthcare facilities, long distances to health facilities, shortage of medical supplies as well as limited insurance coverage. This points to the importance of strengthening system-level factors alongside mechanisms for better financial protection to catalyse the achievement of equity in access to healthcare services.

- $\quad$ Statement 3: While formal health insurance is a necessary and essential factor in improving access to healthcare services in Kenya, it is not sufficient on its own.

Evidence provided in this dissertation shows that formal insurance is a key component to improving equity of access through the improvement of financial risk protection. Household insurance status is seen to be associated with a reduction in socioeconomic inequalities related to $\mathrm{CHE}$. Again, the dissertation observed the reducing effect of insured households on the cost-related unmet need for healthcare services. However, evidence has uncovered the existence of inequalities in insurance coverage, mainly because formal insurance is based on a premium, limiting the ability of the economically disadvantaged to pay. Also, there are psycho-social barriers associated 
with insurance uptake even when premiums are relatively low, mainly centred on individual risk-preferences, trust and perceived health status. This dissertation also notes that non-medical costs, such as transport costs, that are not covered by health insurance significantly contributed to catastrophic payments. Hence, there is a need to devise financial mechanisms that provide protection beyond medical costs. Considering that the majority of the population in Kenya and the region is working informally, there is a growing need to explore innovative approaches through informal networks and mutual organisations that are more contextual in pooling finances and offer financial cushions to the less well-off.

- Statement 4: Vulnerability to shocks limits financially unprotected households' ability to invest in healthcare and other welfare services.

This statement points to the need for collaboration across sectors, and beyond that of health, to address socioeconomic factors that influence the uptake of healthcare services. The evidence in this dissertation shows that rich-poor inequalities, regional disparities and household risks exacerbate inequities in access. Given that some of the drivers of inequities in access extend beyond the healthcare sector, this implies that policies in health systems are fundamentally required to be complemented through other relevant sector mechanisms. For instance, investments in social protection mechanisms would substantially improve financial risk protection among economically disadvantaged households. Although Kenya has made significant progress in putting in place social protection mechanisms, they are limited in coverage due to minimal government investment. Finally, this statement calls for the need to draw efficiencies and synergies across various sectors to accelerate the achievement of equity of access and subsequently, UHC.

- Statement 5: Application of multiple dimensions to measure equity provides a more holistic and nuanced view on equity of access to healthcare services.

A novel contribution of this research was the application of different measures to assess different perspectives of equity in access to healthcare services. While there are various approaches to measure equity of access, this dissertation combined the measures to reflect various perspectives in order to assess equity in access to healthcare services. In addition, it moved beyond just measures of risk protection to the disparities, their drivers and associated risks. For instance, while CHE and its inequalities in Kenya have been assessed to a certain extent, this dissertation is the first to focus on the determinants of inequalities and their changes over time. Furthermore, it also assesses those who refrain from accessing care due to a lack of risk protection and the risks that limit households to access healthcare. Assessing the various perspectives of equity in access may provide detailed evidence on the existing disparities that could then inform better-targeted policy decisions.

This dissertation has some limitations that need to be considered when interpreting the findings. These limitations suggest areas that could inform further 
research on the expansion of the evidence on equity in access to healthcare services in SSA. These include the following:

1. The study used cross-sectional data which is limited in providing depth in analysing data over time. Furthermore, panel data are better suited for this kind of analyses. Hence, future studies would be more beneficial if they provide time series analysis using panel data.

2. This study focused on the demand side of the healthcare system. Although the supply side does influence access to healthcare services, this study was only concerned with individual/household direct access or non-access to healthcare services. Nonetheless, the results hint to the existence of inequities within the supply side of the healthcare system. Hence, further research could consider examining the system-level inequities within the healthcare system that limit access to care and services.

3. The results analysed in this dissertation are based on self-reported healthcare needs and costs by the respondents. This could be subjective given that different individuals have varying perceptions of their healthcare needs, and that healthcare behaviour is complex across different socioeconomic groups. This calls for further research to explore direct causal pathways to access, but also to explore qualitative methodologies to analyse behavioural aspects.

Although the empirical chapters are specific to Kenya, they provide evidence that can be applied and replicated in other countries in SSA. The policy implications derived from the findings of this dissertation include the following:

- Increase investments in healthcare services and social protection measures to reduce reliance on out-of-pocket payments.

Currently, only $6 \%$ of the GDP is allocated to healthcare with OOPs accounting for a third of the health expenditure. Thus, the government needs to mobilise additional resources through counties own resource revenues, and to expand the national insurance coverage to the informal sector with the ability to pay. This would contribute to increase the resource base for investments in the healthcare system, subsequently contributing to improvements in financial risk protection, especially for the most vulnerable.

- Optimise the decentralised healthcare system through better coordination between national and county governments.

This would help to prioritise counties that are lagging behind and hence, reduce disparities across the counties. In addition, the government should fundamentally consider prioritisation of counties based on their needs rather than the performance of specific services, as is the case with the county-level equalisation fund.

- Leverage the informal and mutual networks that are common and contextual in countries that have a large informal workforce, such as Kenya. 
Although formal insurance is vital to improving access, such a policy can be achieved through the establishment of collective-based insurance packages for these mutual networks to expand insurance coverage and thus, improve financial risk protection. Also, the government needs to put in place robust mechanisms that allow for the integration of these mutual organisations and informal networks into the broader health financing systems.

- A multisectoral approach is vital to addressing the socioeconomic inequalities and potential financial risks.

Mechanisms for addressing inequity should not only focus on reducing the direct effects of the determinants. Furthermore, the drivers of inequalities in financial risk like employment and economic status of the household extend beyond the health sector. This requires a wider collaboration and synergies across various government institutions and stakeholders so as to draw possible efficiencies and maximise impact.

In conclusion, this dissertation has assessed more than one perspective of equity in access to healthcare services by applying different measures. It adds to the knowledge and evidence on equity of access by going beyond simply exploring the measures of financial risk protection. This is achieved by examining the socioeconomic inequalities in $\mathrm{CHE}$, the drivers that sustain the inequalities, the effect of variations across Kenyan regions and the risks that may limit households from utilising healthcare services.

Overall, the dissertation underscores the role of financial risk protection as a pathway to achieving equity of access. It emphasises the importance of applying a multisectoral approach to comprehensively address both system- and individual-level factors to achieve equity of access to healthcare services. The recommendations also point to increasing investments in healthcare, as well as social protection to address risks and socioeconomic inequalities that may limit the achievement of UHC in Kenya. In general, for countries such as Kenya, that are challenged with devising mechanisms for equity in access, it calls for a rethinking of the health financing structures and policies that could then provide more equitable access, especially among the poor and most vulnerable. 


\section{Impact statement}

The pursuit of universal health coverage (UHC) has left governments in SubSaharan African countries grappling with the appropriate mechanisms to ensure equity of access to healthcare services. This dissertation provides substantial evidence to shape policy and practice in Kenya and other countries within the region. Kenya has demonstrated commitment to achieving better health for all by prioritising UHC, and in October 2020, launched the roll-out of the UHC programme countrywide. Therefore, this evidence is perfectly timed to inform the UHC programme's implementation and shape equity of access priorities in Kenya.

The dissertation highlights what is known on the extent of the financial risk gaps and the drivers of CHE across SSA countries (Chapter 2). The findings are instrumental for countries currently prioritising UHC to draw lessons on the factors necessary to intensify the implementation. For instance, the review highlights the need to leverage and innovate informal and mutual networks in building financial risk protection mechanisms due to the large informal population in Africa. Furthermore, mutual organisations are more contextual in pooling finances and could help build collectivebased insurance packages to improve coverage.

Kenya continues to suffer from socioeconomic inequalities, as evidenced in this dissertation. The findings stress the existence of inequalities and variations in access to healthcare services across social-economic groups, rural-urban strata and regions or counties (Chapter 3 and 4). The COVID-19 pandemic has exacerbated these disparities in that some regions have better access to testing, treatment and emergency care than others, and only those with the ability to pay have access to the needed care. This dissertation emphasises the need for increased healthcare investment to improve the supply of quality healthcare services to meet all populations' healthcare needs.

In 2013, Kenya decentralised its healthcare system to counties to promote equitable delivery of healthcare services. This dissertation underscores the relevance of decentralised systems as an opportunity to reduce and address disparities in the healthcare system. While the implementation of the decentralised systems is in the early stages in Kenya, this dissertation's findings echo the need for improved resource planning and coordination between the national and county governments for an equitable distribution of resources. The results are also crucial to informing countries that have decentralised structures to optimise these systems as an avenue to maximise impact, and for countries that intend to decentralise, how to strategically decentralise to achieve equity of access.

To the best of my knowledge, this dissertation (Chapter 5) is the first impact assessment of households' economic shocks on healthcare utilisation in Kenya. It, therefore, offers important insights to policymakers on the influence of economic shocks on health-seeking behaviour. Furthermore, the COVID-19 pandemic has triggered unanticipated negative economic shocks on households through the loss of livelihoods. 
The findings highlight the importance of collaboration across sectors to draw synergies and efficiencies for a more holistic approach to improve the population's wellbeing. Also, it draws attention to the need for increased investments in social protection mechanisms that offer a cushion to financial risks and build household's financial resilience over time. The results are expected to provoke the need for a deeper evaluation of economic shocks' causal pathway on the utilisation of healthcare services.

This dissertation demonstrated that evaluating equity of access to healthcare services through multiple measures provides a broader perspective that could better inform policy decisions. Overall, for countries affected by inequities in access to healthcare, the dissertation findings call for rethinking the health policies and financing structures that provide equitable access, especially for the poor and most vulnerable, to accelerate UHC achievement. 


\section{Acknowledgements}

This PhD was similar to a 'race' with pacesetters and those who cheered me on to the finish line. I dedicate this achievement to my dearest mum, friend and greatest cheerleader. You followed every step of it but left just when I got to the end. In my heart you remain forever.

I start by thanking my superb team of supervisors who guided and advised me through this journey. You were very pragmatic; you made this experience so bearable than I could have ever imagined. Indeed "What we learn with pleasure, we never forget" Alfred Mercier.

Prof. Wim, I am so glad our paths crossed. I could never have asked for a better promoter, 'strict' but the kindest person. You left me to freely explore different ideas and find my ground. You challenged me to think 'unconventionally'. Despite working from a distance, your consistent bi-weekly check-ins kept me committed and on-track. I admired your patience and swiftness to read more and more versions of my work.

Thank you to my co-supervisor Dr Jelena, for walking with me from the very early days. This research idea was concretised when we met, you guided me to put my ideas into a coherent research proposal. Thank you for the constant reminders that "I can do it". Also, I appreciate your good advice to throw some fun into this journey - for sure I needed it.

I am grateful to the assessment committee Prof. Franziska Gassman, Prof. Jo Ritzen, Dr Zina Nimeh, Prof. Khama Rogo and Prof. Steven Koch. Thank you for the constructive comments that helped to refine and enhance this dissertation.

To my GPAC colleagues Maria, Gillian, Mauricio, Martha, Richard, Casty and others that I met along the way, thank you for being great company in this journey and for the frequent chats despite being miles apart.

To my friends. Rhoune, I am grateful that you took time to read some of my work and for your consistent encouragement that I will make it to the end. Yvonne, thanks for the countless relaxing wine dates, and for joining me in Amsterdam for some fun. Prof. Ikamari, Dr Yaw Afrane, Prof. Appiah-Poku, thank you for checking on my progress even when I was too busy to keep in touch.

Thank you to Dr Mindel and the GPAC coordination team for making every visit to Maastricht seamless. I appreciate all the UNU-MERIT colleagues who participated in my presentation over the years; your insights helped shape this dissertation. I cannot forget the warm hospitality of the Dutch people who hosted me during my numerous trips. You all made my experience in the Netherlands so wonderful. I will miss every bit of it, especially the Stroopwafels. 
The data utilised in this dissertation comes from the Kenya National Bureau of Statistics (KNBS). I appreciate the swiftness of the data team in replying to my requests, and the willingness to respond to my additional clarifications - Keep up the good work. 


\section{Biography}

Purity Njagi was born in Nairobi, Kenya in 1981. In 2004, she obtained a Bachelor of Education degree in Economics from the University of Nairobi. In 2007 she completed a Master of Arts in Population Studies from the Population Studies and Research Institute(PSRI); and later a Master of Science in Medical Statistics from the Institute of Tropical and Infectious Diseases(UNITID) in 2011.

She started her professional career as a data analyst working in reproductive health programmes with Deutsche Stiftung Weltbevölkerung (DSW). She then worked with AMREF Health Africa as a health information systems officer. Later, she joined Care International and Management Sciences for Health (MSH) respectively providing technical assistance to national health CSOs on monitoring and evaluation for HIV programmes. Prior to commencing her PhD, she was the monitoring and evidence advisor for a Multi-country Malaria programme in East and Southern Africa region with Population Services International (PSI) .

Alongside her $\mathrm{PhD}$, she consulted for multiple organisations including: The Global Fund for HIV, TB and Malaria, conducting M\&E systems assessments and data quality reviews in Namibia, Uganda and Rwanda. The Population Council supporting monitoring and operations research for gender programming in Ethiopia and Somali. GAVI, where she assessed the performance of the immunisation programme and vaccine supply chain in Rwanda. UK Department for International Development (DFID), where she developed the monitoring and learning framework for the Somalia Health and Nutrition programme.

Between 2018-2020, she consulted for UN-Habitat as the results and measurement expert providing technical assistance to urban basic services programmes for displacement affected communities in Somalia. Also, during her PhD she conducted a systematic review on the economic costs of infertility treatment for patients in Low-and Middle-Income countries financed by the WHO. 


\section{Publications}

1. Njagi, P., Arsenijevic, J., \& Groot, W. (2020). Decomposition of changes in socioeconomic inequalities in catastrophic health expenditure in Kenya. PLOS ONE, 15(12), e0244428. https://doi.org/10.1371/journal.pone.0244428

2. Njagi, P., Groot, W., Arsenijevic, J., Dyer, S., Mburu, G., \& Kiarie, J. (2020). Economic costs of infertility care for patients in low-income and middle-income countries: A systematic review protocol. BMJ Open, 10(11), e042951. https://doi.org/10.1136/bmjopen-2020-042951

3. Njagi Purity, Arsenijevic Jelena, Groot Wim 2020; Cost-related unmet need for healthcare services in Kenya. BMC Healthcare services Research, 20(1). https://doi.org/10.1186/s12913-020-05189-3

4. Njagi Purity, Arsenijevic Jelena, Groot Wim 2018; Understanding variations in catastrophic health expenditure, its underlying determinants and impoverishment in Sub-Saharan African countries: a scoping review. BMC Systematic Reviews Journal. https://doi.org/10.1186/s13643-018-0799-1

5. Rhoune Ochako, Dunstone Ulwodi, Purity Njagi, Steven Kimetu and Aggrey Onyango 2011; Trends and determinants of Comprehensive HIV and AIDS knowledge among urban young women in Kenya, AIDS Research and Therapy 2011, 8:11 http://www.aidsrestherapy.com/content/8/1/11 



\section{UNU-MERIT/MGSoG Dissertation Series}

2021

\author{
María José Espinosa-Romero \\ The Shadow of Hierarchy in Marine \\ Fisheries Governance \\ UNU-MERIT/MGSoG Dissertation \\ Series № 257
}

\section{Lorena Rivera León}

Unveiling the Determinants of Scientific

Productivity in Middle-Income Countries:

An Economics of Science Perspective

UNU-MERIT/MGSoG Dissertation

Series № 256

\section{Racky Balde}

Essays on Informal versus formal Economy Choices

UNU-MERIT/MGSoG Dissertation

Series № 255

\section{Caio Torres Mazzi}

Learning, Capabilities and Governance in

Global Value Chains

UNU-MERIT/MGSoG Dissertation

Series № 254

\section{Giulia Rossello}

Social Transformations and Labour Market Entry

An Investigation into University Systems in Emerging Economies

UNU-MERIT/MGSoG Dissertation

Series № 253
2020

\section{Rose Camille Vincent}

Essays in Public Economics

Multi-Layer Tax Structure and

Implications

UNU-MERIT/MGSoG Dissertation

Series № 252

\section{Emmanuel Mensah}

Structural change in developing countries: patterns, causes, and consequences UNU-MERIT/MGSoG Dissertation Series № 251

\section{Ornsaran Manuamorn}

Governance of International Adaptation Finance for Local Climate Change Adaptation: An Analysis of Adaptation Fund Projects

UNU-MERIT/MGSoG Dissertation

Series № 250

\section{Gillian McFarland}

Doing policy in Further Education An exploration of the enactment of the GCSE resits policy in Further Education colleges in England

UNU-MERIT/MGSoG Dissertation

Series № 249

\section{Omar Rodriguez Torres}

Essays on Entrepreneurship in Colombia UNU-MERIT/MGSoG Dissertation Series № 248

Elaine Lebon-McGregor International Organizations and Global Migration Governance UNU-MERIT/MGSoG Dissertation Series № 247 
Janyl Moldalieva

Playing the 'Game'of Transparency and

Accountability in Kyrgyzstan's Resource

Governance

UNU-MERIT/MGSoG Dissertation

Series № 246

Kaleab Kebede Haile

Essays on Rural Household Decision-

Making under Climate Risk

UNU-MERIT/MGSoG Dissertation

Series № 245

Nora Jasmin Ragab

Diaspora Mobilisation in a Conflict Setting

UNU-MERIT/MGSoG Dissertation

Series № 244

\section{Mary Kaltenberg}

From Micro to Macro: Essays on

Technological Change and Income

Divergence

UNU-MERIT/MGSoG Dissertation

Series № 243

\section{Ayla E. Bonfiglio}

Student migrant, refugee or both?

Exploring Refugee Agency and Mobility

through Tertiary Education in Kenya,

South Africa and Uganda

UNU-MERIT/MGSoG Dissertation

Series № 242

\section{Danilo Sartorello Spinola}

Cycles, Economic Structures and External Constraints.

A Structuralist study on the causes of economic volatility in Latin America

UNU-MERIT/MGSoG Dissertation

Series № 241
2019

\section{Jemal Adem}

Livelihood Vulnerability to Shocks,

Behaviour and Investment in Education:

Essays in Behavioural Development

Economics

UNU-MERIT/MGSoG Dissertation

Series № 240

\section{Davina Osei}

Corrupt or corrupted networks?

An empirical enquiry

UNU-MERIT/MGSoG Dissertation

Series № 239

\section{Patima Chongcharoentanawat}

Beyond Static Inequality

Public policies and economic mobility in

Thailand

UNU-MERIT/MGSoG Dissertation

Series № 238

Charlotte Guillard

Rethinking economic growth

and structural change.

The role of boundaries and linkages between

industries

UNU-MERIT/MGSoG Dissertation

Series № 237

\section{Nicolas Echarti}

Employment Effects or Vocational

Rehabilitation in Germany:

A quantitative analysis

UNU-MERIT/MGSoG Dissertation

Series № 236

\section{Shellie E. Solomon}

Neighborhoods Matter:

Crime, collective efficacy and foreclosures

in Miami

UNU-MERIT/MGSoG Dissertation

Series № 235 


\section{Michał Kazimierczak}

Regional Innovation and Entrepreneurship Patents, trade marks, entry and entrants' growth in European manufacturing industries

UNU-MERIT/MGSoG Dissertation Series № 234

\section{Fernanda Soares}

The Influence of Within School and Across Schools' Collaborative Practices on Student Learning and Teaching Outcomes in West Africa

UNU-MERIT/MGSoG Dissertation

Series № 233

\section{Mira Bierbaum}

New Mindsets to Innovate Activation UNU-MERIT/MGSoG Dissertation Series № 232

\section{Norman Dytianquin}

Technology in the Asian Miracle and Crisis Debates: Applications of and Insights from the Field of Influence Approach to InputOutput Analysis

UNU-MERIT/MGSoG Dissertation Series № 231

\section{Nga Le}

The implications of health insurance for the labour market and patient satisfaction with medical care in Vietnam

UNU-MERIT/MGSoG Dissertation

Series № 230

\section{Jinhyuck Park}

Intellectual Property right protection and cross-border RED investments by multinational enterprises UNU-MERIT/MGSoG Dissertation

Series № 229

\section{Richard de Groot}

Show me the Money:

Essays on the Impact of Cash Transfers on Child Nutrition and the Role of IntraHousehold Dynamics

UNU-MERIT/MGSoG Dissertation Series № 228

\section{Catie Lott}

Diamonds are a Women's Best Friend Broadening Measures of Women's Access to Formal Political Decision-Making UNU-MERIT/MGSoG Dissertation Series № 227

\section{Ana Cristina Calderon Ramirez}

Public Management Reforms

Three stories about public procurement agencification in Latin America UNU-MERIT/MGSoG Dissertation Series № 226

\section{Camilo Nicanor Carrillo Purin}

Teachers' in-service training and student achievement:

The effect of in-service training of Peruvian teachers on student achievement

UNU-MERIT/MGSoG Dissertation

Series № 225

\section{Hugo Confraria}

Developing scientific capacity in the Global South

UNU-MERIT/MGSoG Dissertation

Series № 224

Alison Cathles

Educational Pathways and Skills:

Past, Present, and Future

UNU-MERIT/MGSoG Dissertation

Series № 223 


\section{Ibrahima Sory Kaba}

Aggregate Fluctuations and Development:

Essays on Macroeconomic Volatility and

Economic Growth

UNU-MERIT/MGSoG Dissertation

Series № 222

\section{Charlotte Keijser}

Firm Participation, Learning and

Innovation in Heterogenous Value Chains

of IT-enabled Services

UNU-MERIT/MGSoG Dissertation

Series № 221

\section{Salih Çevikarslan}

Innovation Strategies and Their

Implications for Technological Change and Market Outcomes:

An Evolutionary Multi-Agent Based

Modelling Approach

UNU-MERIT/MGSoG Dissertation

Series № 220

\section{Wondimagegn Mesfin Tesfaye}

Essays on the Impacts of Climate-Smart

Agricultural Innovations on Household

Welfare

UNU-MERIT/MGSoG Dissertation

Series № 219

\section{Tatevik Poghosyan}

How Board Networks Affect Firm

Performance and Innovation Incentives in

Transition Economies: The Case of

Armenia

UNU-MERIT/MGSoG Dissertation

Series № 218

\section{Arip Muttaqien}

Essays on Inequality and Polarization:

Empirical Studies in Developing Asia

UNU-MERIT/MGSoG Dissertation

Series № 217

\section{Katrin Marchand}

Essays on Forced Migration and Labour

Market Participation in Developing

Countries

UNU-MERIT/MGSoG Dissertation

Series № 216

\section{Ortrun Merkle}

The Myth of Gender Neutral Power:

Corruption and Gender Norms

UNU-MERIT/MGSoG Dissertation

Series № 215

\section{Biljana Meshkovska}

Life after Trafficking:

(re)integration processes of women that have been trafficked for the purpose of sexual exploitation in Europe

UNU-MERIT/MGSoG Dissertation

Series № 214

\section{Vincenzo Vinci}

The Relevance of Institutions and People's

Preferences for Social Protection

UNU-MERIT/MGSoG Dissertation

Series № 213

\section{Silke Heuser}

The Effectiveness of Environmental Policies on Reducing Deforestation in the Brazilian Amazon

UNU-MERIT/MGSoG Dissertation

Series № 212

\section{Jennifer Waidler}

Social Assistance and Remittances and Their Role in the Fight Against Poverty UNU-MERIT/MGSoG Dissertation Series № 211 


\section{Choolwe Muzyamba}

The role of community mobilization in the promotion of maternal health of women

living with HIV in Zambia

UNU-MERIT/MGSoG Dissertation

Series № 210

\section{Juan Carlos A. Castillo Sánchez}

Assessing the Role of the Export Sector in

Mexican Economic Development,1965-

2014

UNU-MERIT/MGSoG Dissertation

Series № 209

\section{Tareq Abuelhaj}

Food Security Policy Impact Analysis: The

Econometrics of Cash and Food Assistance

Cost Effectiveness

UNU-MERIT/MGSoG Dissertation

Series № 208

Marta Férnandez de Arroyabe Arranz

Essays on MEAS and Innovation

UNU-MERIT/MGSoG Dissertation

Series № 207

\section{Clotilde Mahé}

Essays on Migration and Occupational

Choice

UNU-MERIT/MGSoG Dissertation

Series № 206

\section{Simone Sasso}

Talent on the move. Essays on Human

Capital, Graduate Mobility and Economic

Development

UNU-MERIT/MGSoG Dissertation

Series № 205

Khaled Walid Rajab

Strategic Planning under Fragility

UNU-MERIT/MGSoG Dissertation

Series № 204
Mutinta Hambayi Nseluke

A Tall Order: Improving Child Linear

Growth

UNU-MERIT/MGSoG Dissertation

Series № 203

\section{Elvis Korku Avenyo}

Innovations and Firm Performance

in sub-Saharan Africa: Empirical Analyses

UNU-MERIT/MGSoG Dissertation

Series № 202

\section{Ni Zhen}

Employment Dynamics, Firm Performance and Innovation Persistence in the Context of Differentiated Innovation Types:

Evidence from Luxembourg

UNU-MERIT/MGSoG Dissertation

Series № 201

\section{Caroline Wehner}

Too Scared to Achieve: The Relation

Between Neuroticism, Conscientiousness

and Socioeconomic Outcomes

UNU-MERIT/MGSoG Dissertation

Series № 200

\section{Stefania Innocenti}

On Institutional Persistence

UNU-MERIT/MGSoG Dissertation

Series № 199

\section{Hassen Abda Wako}

Economic Globalization, Institutions and

Development: Essays on Aid, Foreign

Direct Investment and Trade

UNU-MERIT/MGSoG Dissertation

Series № 198

\section{7}

\section{Hans-Erik Edsand}

Winds of Change

UNU-MERIT/MGSoG Dissertation

Series № 197 


\section{Ana Patricia Silva Vara}

Redressing the Gender Gap

UNU-MERIT/MGSoG Dissertation

Series № 196

\section{Andrés Iván Mideros Mora}

Essays on the Economic Effects of Noncontributory Social Protection

UNU-MERIT/MGSoG Dissertation

Series № 195

\section{Tobias Broich}

New Actors in the Global Economy

UNU-MERIT/MGSoG Dissertation

Series № 194

\section{Bernard Nikaj}

From No-government to E-government UNU-MERIT/MGSoG Dissertation

Series № 193

\section{Ali Safarnejad}

Prioritizing the HIV Response

UNU-MERIT/MGSoG Dissertation

Series № 192

\section{Clovis Freire}

Diversification and Structural Economic

Dynamics

UNU-MERIT/MGSoG Dissertation

Series № 191

\section{Michael Verba}

Innovation and Knowledge Dynamics:

Essays on the Knowledge Economy

UNU-MERIT/MGSoG Dissertation

Series № 190

\section{Pui Hang Wong}

The Hearts and Minds in Conflict and

Peace: The Economics of

Counterinsurgency and the Psychology of

Reconstruction

UNU-MERIT/MGSoG Dissertation

Series № 189

\section{Brenda Yamba}

Schooling Despite All Odds: Evidence from Lesotho on Female Child Carers who Stayed in School

UNU-MERIT/MGSoG Dissertation

Series № 188

\section{Sheng Zhong}

Moving towards An Energy Efficient

Future: Essays on Energy Efficiency,

Technology and Development

UNU-MERIT/MGSoG Dissertation

Series № 187

\section{Julieta Marotta}

Access to Justice and Legal Empowerment of Victims of Domestic Violence through Legal Organizations in the City of Buenos Aires: A Qualitative Empirical Legal Study UNU-MERIT/MGSoG Dissertation Series, № 186

\section{Andrea Franco-Correa}

On the Measurement of Multidimensional Poverty as a Policy Tool: Empirical Applications to Chile, Colombia, Ecuador and Peru

UNU-MERIT/MGSoG Dissertation Series, № 185

\section{6}

\section{Yesuf Awel}

Insurance for Growth: Empirical Essays on Insurance Demand and Impacts in Africa UNU-MERIT Dissertation Series, № 108 
Tigist Mekonnen Melesse

Grow More Food using Fewer Resources:

Agricultural Technology Adoption and

Innovation Practices for Inclusive and

Sustainable Development

UNU-MERIT Dissertation Series, № 107

\section{Eleni Yitbarek}

Getting Ahead or left Behind? Essays on Poverty Dynamics and Social Mobility in Africa

UNU-MERIT Dissertation Series, № 106

\section{Thuy Dieu Nguyen}

Firm-Level Theory and Evidence of

Corruption

UNU-MERIT Dissertation Series, № 105

\section{Raquel Tsukada Lehman}

Essays on Household Production with Labor-Saving Technology

UNU-MERIT Dissertation Series, № 104

\section{Eva Barteková}

Multi-Problem Challenges for a Renewable Future: Empirical Studies on Competitive

Disadvantages from Electricity Price

Differentials and Mineral Supply Risk in

an Open Economy

UNU-MERIT Dissertation Series, № 103

\section{Jocelyn Olivari}

Entrepreneurial Traits and Innovation:

Evidence from Chile

UNU-MERIT Dissertation Series, № 102

\section{Muhammad Shafique}

Essays on the role of knowledge, RED, and

Technology-based Firms in the Evolution of Socio-techno-economic System

UNU-MERIT Dissertation Series, № 101

\section{Serdar Türkeli}

Governance of Innovation Policy:

Empirical Studies on Applied Political

Economy by Multi-Methods Analysis

UNU-MERIT Dissertation Series, № 100

\section{Ayokunu Adedokun}

Pathways to Sustainable Peace building in Divided Societies: Lessons and Experiences from Mozambique

MGSoG Dissertation Series, № 75

\section{Luiz Rothier Bautzer}

Organizing Concurrent Engineering

through ICT Platforms

Blueprinting Product Lifecycle

Management Platforms across Disciplinary Agencies

MGSoG Dissertation Series, № 74

\section{Natalia Popova}

Migration in the Periphery of the European Union:

Determinants of Successful and Sustainable Labour Market Integration of Return Migrants in Albania, Egypt, Moldova and Tunisia

MGSoG Dissertations Series, № 73

\section{Richard A. Martina}

Uncertainty and Resource Constraint in the Small Island Developing States:

Essays in Entrepreneurial Cognition MGSoG Dissertations Series, № 72 
Cécile Cherrier

The Expansion of Basic Social Protection in Low-income Countries:

An Analysis of Foreign Aid Actors' Role in the Emergence of Social Transfers in Sub-

Saharan Africa

MGSoG Dissertations series, № 71

\section{Paul Caldron}

The Tacit Bargain in Short-Term Medical Missions: Why U.S. physicians go and what it costs

MGSoG Dissertation Series, № 70

\section{Mahmut Kobal}

Customs E Excellence: A Comparative Approach on Administrative and Regulatory Compliance Perspectives of the EU-Turkey Customs Union

MGSoG Dissertation Series, № 69

\section{Craig Loschmann}

Essays on Conflict-related Migration and Development in the Case of Afghanistan MGSoG Dissertations Series, № 68

\section{Andrea Milan}

Rural Livelihoods, Location and Vulnerable Environments: Approaches to Migration in Mountain areas of Latin America MGSoG Dissertation Series, № 67

\section{Farida Lada}

On Guarding the Welfare of Clinical Trial Subjects While Promoting Novel Drug Innovation A Game Theoretical Approach MGSoG Dissertation Series, № 66
Hibret Belete Maemir

Dissecting Aggregate Productivity:

International Integration and Growth with Heterogeneous Firms

UNU-MERIT Dissertation Series, № 96

\section{Giorgio Triulzi}

Looking for the Right Path: Technology

Dynamics, Inventive Strategies and

Catching-up in the Semiconductor

Industry

UNU-MERIT Dissertation Series, № 95

\author{
Abdul Baseer Qazi \\ Knowledge flows and networks in the ICT \\ sector: The case of Pakistan \\ UNU-MERIT Dissertation Series, № 94
}

\section{Ajay Thutupalli}

Technology Paradigm Shifts in

Agriculture: Drivers of Sustainability and Catch up

UNU-MERIT Dissertation Series, № 93

\section{Eduardo Urias}

Improving access to HIVIAIDS treatment in Brazil: When are Compulsory Licenses effective in Price Negotiations?

UNU-MERIT Dissertation Series, № 92

\section{Francesca Guadagno}

Why have so few Countries Industrialised? UNU-MERIT Dissertation Series, № 91

\section{Daniel Opolot}

The Evolution of Beliefs and Strategic Behaviour

UNU-MERIT Dissertation Series, № 90 
Alejandro Lavopa

Structural Transformation and Economic Development: Can Development Traps be Avoided

UNU-MERIT Dissertation Series, № 89

\section{Jinjin Zhao}

Urban water management reform: The Case of China

UNU-MERIT Dissertation Series, № 88

\section{Simona Vezzoli}

Borders, Independence and Post-colonial

Ties: the Role of the State in Caribbean

Migration

MGSoG Dissertation Series, № 65

\section{Silvia Consuelo Gómez Soler}

Civil Conflict and Education: How Does

Exposure to Civil Conflict Affect Human

Capital Accumulation? Evidence from

Standardized Exit Exams in Colombia

MGSoG Dissertation Series, № 64

\section{Paula Nagler}

Occupational Choice in the Developing

World

MGSoG Dissertation Series, № 63

\section{Jasmin Kientzel}

Determinants of Professional Commitment

to Environmental Sustainability

MGSoG Dissertation Series, № 62

\section{Mehmet Güney Celbiş}

Regional Policies: Convergence, Trade, and the Allocation of Public Capital

MGSoG Dissertation Series, № 61

\section{Florian Henning}

Living Up to Standard: Interoperability Governance and Standards Adoption in Government Information Networks MGSoG Dissertation Series, № 60
Niels P. Groen

The Never-Ending Project

Understanding E-Government Project

Escalation

MGSoG Dissertation Series, № 59

\section{Derek Copp}

Teacher-Based Reactivity to Provincial

Large-scale Assessment in Canada

MGSoG Dissertation Series, № 58

\section{Michaella Vanore \\ Family-Member Migration and the \\ Psychosocial Health Outcomes of Children in Moldova and Georgia \\ MGSoG Dissertation Series, № 57}

\section{Sonja Fransen}

The Economic and Social Effects of Remittances and Return Migration in Conflict-Affected Areas: The Case of Burundi

MGSoG Dissertation Series, № 56

\section{Ibrahim Khalil Conteh}

The Impact of Floods on Primary School

Education in Zambia

MGSoG Dissertation Series, № 55

\section{Richard Bluhm}

Growth Dynamics and Development Essays in Applied Econometrics and

Political Economy

MGSoG Dissertation Series, № 54

\section{Nevena P. Zhelyazkova}

Work-Family Reconciliation and Use of Parental Leave in Luxembourg: Empirical Analysis of Administrative Records

MGSoG Dissertation Series, № 53 
2014

\section{Dirk Crass}

The Impact of Brands on Innovation and

Firm Performance: Empirical Evidence

from Germany

UNU-MERIT Dissertation Series, № 87

\section{Samyukta Bhupatiraju}

The Geographic Dimensions of Growth and Development

UNU-MERIT Dissertation Series, № 86

\section{François Lafond}

The Evolution of Knowledge Systems

UNU-MERIT Dissertation Series, № 85

\section{Annalisa Primi}

Promoting Innovation in Latin America:

What Countries Have Learned (and What

They Have Not) in Designing and

Implementing Innovation and Intellectual

Property Policies

UNU-MERIT Dissertation Series, № 84

\section{Fatoumata Lamarana Diallo}

Evaluation of Meal and Deworming

Programs for Primary Schools in Rural

Senegal

UNU-MERIT Dissertation Series, № 83

\section{Sachin Kumar Badkas}

Metachoice and Metadata: Innovating with Environmental Policy Analysis in Europe MGSoG Dissertation Series, № 52

\section{Irina S. Burlacu}

An Evaluation of Tax-Benefit Systems Impact on the Welfare of Frontier Worker: The Case of Luxembourg and Belgium MGSoG Dissertation Series, № 51

\section{Özge Bilgili}

Simultaneity in Transnational Migration Research: Links Between Migrants' Host and Home Country Orientation MGSoG Dissertation Series, № 50

\section{Yulia Privalova Krieger}

Reshaping the Big Agenda: Transnational Politics and Domestic Resistance Financial crisis and social protection reform in Bosnia and Herzegovina

MGSoG Dissertation Series, № 49

\section{Marieke van Houte}

Moving Back or Moving Forward? Return migration after Conflict

MGSoG Dissertation Series, № 48

\section{Oxana Slobozhan}

Global Governance in the Management of Natural Resources: The Case of the Extractive Industries Transparency Initiative (EITI)

MGSoG Dissertation Series, № 47

\section{Luis Bernardo Mejia Guinand}

The Changing Role of the Central Planning Offices in Latin America: A Comparative Historical Analysis Perspective (19502013)

MGSoG Dissertation Series, № 46

\section{Cheng Boon Ong}

Ethnic Segregation in Housing, Schools and Neighbourhoods in the Netherlands MGSoG Dissertation Series, № 45

\section{Luciana V. Cingolani}

Bureaucracies for Development: Oxymoron or Reality? Studies on State Capacity in Challenging Governance Contexts MGSoG Dissertation Series, № 44 


\section{Carlos Cadena Gaitán}

Green Politics in Latin American Cities -

Sustainable Transport Agendas

MGSoG Dissertation Series, № 43

\section{Katie Kuschminder}

Female Return Migration and

Reintegration Strategies in Ethiopia

MGSoG Dissertation Series, № 42

\section{Metka Hercog}

Highly-Skilled Migration and New

Destination Countries

MGSoG Dissertation Series, № 41

\section{Margaret Agaba Rugadya}

Can Remittances Influence the Tenure and

Quality of Housing in Uganda?

MGSoG Dissertation Series, № 40

\section{Ilire Agimi}

New Governance Under Limited Statehood:

The Case of Local Government Reform in

Kosovo

MGSoG Dissertation Series, № 39

2013

\section{Anant Kamath}

Information Sharing through Informal

Interaction in Low-Tech Clusters

UNU-MERIT Dissertation Series, № 82

\section{Flavia Pereira de Carvalho}

What we talk about when we talk about Brazilian Multinationals: An Investigation on Brazilian FDI, Economic Structure, Innovation and the Relationship between them

UNU-MERIT Dissertation Series, № 81

\author{
Jun Hou \\ Complementarity in Innovation and \\ Development: A Cross-country \\ Comparison \\ UNU-MERIT Dissertation Series, № 80
}

\section{Rufin Baghana \\ Impacts of Government Incentives to RED, Innovation and Productivity: \\ A Microeconometric Analysis of the \\ Québec Case \\ UNU-MERIT Dissertation Series, № 79}

\section{Lilia I. Stubrin}

High-Tech Activities in Emerging

Countries: A Network perspective on the

Argentinean Biotech Activity

UNU-MERIT/MGSoG Dissertation

Series, № 78

\section{Kristine Farla}

Empirical Studies on Institutions, Policies and Economic Development

MGSoG Dissertation Series, № 38

\section{Marina Petrovic}

Social Assistance and Activation in the Pursuit of Happiness: Shedding New Light on Old Policy Solutions to Social Exclusion MGSoG Dissertation Series, № 37

\section{Laura Torvinen}

Assessing Governance Assessments: The Case of Mozambique: Governance Assessments in the Context of Aid Effectiveness Discourse

MGSoG Dissertation Series, № 36

\section{Biniam Egu Bedasso}

Institutional Change in the Long Shadow of Elite: Essays on Institutions, Human

Capital and Ethnicity in Developing

Countries

MGSoG Dissertation Series, № 35 
Sepideh Yousefzadeh Faal Deghati

Childhoods Embargoed: Constructing and

Reconstructing Multidimensional Child

Poverty in Iran 1984-2009

MGSoG Dissertation Series, № 34

\section{Robert Bauchmüller}

Investing in Early Childhood Care and

Education: The Impact of Quality on

Inequality

MGSoG Dissertation Series, № 33

\section{Martin Rehm}

Unified Yet Separated: Empirical Study on the Impact of Hierarchical Positions within Communities of Learning

MGSoG Dissertation Series, № 32

2012

\section{Abdul Waheed}

Innovation Determinants and Innovation

as a Determinant: Evidence from

Developing Countries

UNU-MERIT Dissertation Series, № 77

\section{Bilal Mirza}

Energy Poverty and Rural Energy Markets in Pakistan

UNU-MERIT Dissertation Series, № 76

\section{Benjamin Engelstätter}

Enterprise Software and Video Games: An Empirical Analysis

UNU-MERIT Dissertation Series, № 75

\section{Fulvia Farinelli}

Natural Resources, Innovation and Export Growth: The Wine Industry in Chili and Argentina

UNU-MERIT Dissertation Series

\section{Rodolfo Lauterbach}

Innovation in Manufacturing: From

Product Variety and Labor Productivity

Growth to Economic Development in Chile

UNU-MERIT Dissertation Series

\section{Kirsten Wiebe}

Quantitative Assessment of Sustainable

Development and Growth in Sub-Saharan Africa

UNU-MERIT Dissertation Series, № 74

\section{Julio Miguel Rosa}

Organizational Strategies, Firms'

Performance and Spatial Spillovers: The

Canadian Case in Research and

Development.

UNU-MERIT Dissertation Series, № 73

\section{Johannes Wilhelmus Marie Boels}

Joseph Schumpeter, Honderd Jaar

Economische Ontwikkeling: Een

Historisch-theoretische Beschouwing.

UNU-MERIT Dissertation Series

\section{Dorcas Mbuvi}

Utility Reforms and Performance of the

Urban Water Sector in Africa

MGSoG Dissertation Series, № 31

\section{Lina Salanauskaite}

Distributional Impacts of Public Policies:

Essays in Ex-Ante and Ex-Post Evaluation

MGSoG Dissertation Series, № 30

\section{Esther Schüring}

To Condition or not - is that the Question? An Analysis of the Effectiveness of Ex-Ante and Ex-Post Conditionality in Social Cash Transfer Programs

MGSoG Dissertation Series, № 29 


\section{Joe Abah}

Strong Organisations in Weak States:

Atypical Public Sector Performance in

Dysfunctional Environments

MGSoG Dissertation Series, № 28

\section{Zina Samih Nimeh}

Social Citizenship Rights: Inequality and

Exclusion

MGSoG Dissertation Series, № 27

2011

\section{Daniel Vertesy}

Interrupted Innovation: Emerging

Economies in the Structure of the Global

Aerospace Industry

UNU-MERIT Dissertation Series, № 72

\section{Tina Saebi}

Successfully Managing Alliance Portfolios:

An Alliance Capability View

UNU-MERIT Dissertation Series, № 71

\section{Nora Engel}

Tuberculosis in India: A Case of Innovation and Control

UNU-MERIT/MGSoG Dissertation

Series, № 70

\section{Evans Mupela}

Connectivity and growth in Sub-Saharan

Africa: The Role of Communication

Satellites

UNU-MERIT Dissertation Series, № 69

\section{Nantawan Kwanjai}

Cross Cultural Intelligence amid Intricate

Cultural Webs: A Tale of the

UnDutchables in the Land of 1002 Smiles

UNU-MERIT Dissertation Series, № 68

\section{Lina Sonne}

Innovation in Finance to Finance

Innovation: Supporting Pro-poor

Entrepreneur-based Innovation

UNU-MERIT Dissertation Series, № 67

\section{Lenka Eisenhamerová}

Legitimacy of 'Humanitarian Military

Intervention'

MGSoG Dissertation Series, № 26

\section{Sonila Tomini}

Informal Payments for Health Care

Services in Albania

MGSoG Dissertation Series, № 25

\section{Jinjing Li}

Dynamic Microsimulation in Public Policy

Evaluation

MGSoG Dissertation Series, № 24

\section{Aziz Atamanov}

Rural Nonfarm Employment and International Migration as Alternatives to Agricultural Employment: The Case of

Kyrgyzstan

MGSoG Dissertation Series, № 23

\section{Frieda Vandeninden}

Poverty Alleviation: Aid and Social

Pensions

MGSoG Dissertation Series, № 22

\section{Juliana Nyasha Tirivayi}

The Welfare Effects of Integrating AIDS

Treatment with Food Transfers: Evidence from Zambia

MGSoG Dissertation Series, № 21

\section{Agnieska Ewa Sowa}

Who's Left Behind? Social Dimensions of Health Transition and Utilization of

Medical Care in Poland

MGSoG Dissertation Series, № 20 


\section{Emmanaouil Sfakianakis}

The Role of Private Actors in the Provision of Public Goods with Applications to Infrastructure and Financial Stability MGSoG Dissertation Series, № 19

\section{Siu Hing Lo}

White Collars Green Sleeves: An Interorganizational Comparison of

Determinants of Energy-Related Behaviors among Office Workers

MGSoG Dissertation Series, № 18

\section{Treena $W u$}

Constraints to Human Capital Investment in Developing Countries:

Using the Asian Financial Crisis in Indonesia as a Natural Experiment

MGSoG Dissertation Series, № 17

\section{Henry Espinoza Peña}

Impact Evaluation of a Job-Training

Programme for Disadvantaged Youths:

The Case of Projoven

MGSoG Dissertation Series, № 16

2010

\section{Fernando Santiago}

Human Resources Management Practices and Learning for Innovation in Developing Countries: Pharmaceutical Firms in Mexico

UNU-MERIT Dissertation Series, № 66

\section{Zakaria Babutsidze}

Essays on Economies with Heterogeneous Interacting Consumers

UNU-MERIT Dissertation Series, № 65

\section{Bertha Vallejo}

Learning and Innovation Under Changing Market Conditions: The Auto Parts Industry in Mexico UNU-MERIT Dissertation Series, № 64
Donatus Ayitey

Technical Change, Competitiveness and Poverty Reduction: A Study of the

Ghanaian Apparel Industry

UNU-MERIT Dissertation Series, № 63

\section{Sergey Filippov}

Multinational Subsidiary Evolution:

Corporate Change in New EU Member

States

UNU-MERIT Dissertation Series, № 62

\section{Asel Doranova}

Technology Transfer and Learning under the Kyoto Regime: Exploring the

Technological Impact of CDM Projects in Developing Countries

UNU-MERIT Dissertation Series, № 61

\section{Florian Tomini}

Between Family and Friend:

Understanding the Interdependency of

Private Transfers

MGSoG Dissertation Series, № 15

\section{Michał Polalowski}

The Institutional Transformation of Social Policy in East Central Europe: Poland and Hungary in Comparative and Historical Perspective

MGSoG Dissertation Series, № 14

\section{Maha Ahmed}

Defining, Measuring and Addressing Vulnerability: The Case of Post Conflict Environments

MGSoG Dissertation Series, № 13

\section{Pascal Beckers}

Local Space and Economic Success: The Role of Spatial Segregation of Migrants in the Netherlands

MGSoG Dissertation Series, № 12 
Victor Cebotari

Conflicting Demands in Ethnically Diverse

Societies: Ethno political Contention and

Identity Values in Europe

MGSoG Dissertation Series, № 11

\section{Dennis Gyllensporre}

Competing and Complementary

Perspectives on the EU as a Crisis

Management Actor:

An Examination of the Common Security and Defence Policy through the Lenses of Idealism and Realism

MGSoG Dissertation Series, № 10

\section{Judit Vall Castello}

Business Cycle and Policy Effects on

Labour Market Transitions of Older and

Disabled Workers in Spain

MGSoG Dissertation Series, № 9

\section{Keetie Roelen}

False Positives or Hidden Dimensions: The

Definition and Measurement of Child

Poverty

MGSoG Dissertation Series, № 8

\section{Denisa Maria Sologon}

Earning Dynamics in Europe

MGSoG Dissertation Series, № 7

\section{Melissa Siegel}

Money and Mobility: Migration and

Remittances

MGSoG Dissertation Series, № 6

\section{Jessica S. Hagen-Zanker}

Modest Expectations: Causes and Effects of Migration on Migrant Households

inSource Countries

MGSoG Dissertation Series, № 5
2009

\author{
Alexis Habiyaremye \\ From Primary Commodity Dependence to \\ Diversification and Growth: Absorptive \\ Capacity and Technological Catch Up in \\ Botswana and Mauritius. \\ UNU-MERIT Dissertation Series, № 60

\section{Yoseph Getachew} \\ The Role of Public Capital in Economic \\ Development
}

UNU-MERIT Dissertation Series, № 59

\section{Sandra Leitner}

Embodied Technological Change and

Patterns of Investment in Austrian

Manufacturing

UNU-MERIT Dissertation Series, № 58

\section{Semih Akçomak}

The Impact of Social Capital on Economic and Social Outcomes

UNU-MERIT Dissertation Series, № 57

\section{Abraham Garcia}

The Role of Demand in Technical Change

UNU-MERIT Dissertation Series, № 56

\section{Saurabh Arora}

Coherence in Socio-technical Systems: A

Network Perspective on the Innovation

Process

UNU-MERIT Dissertation Series, № 55

\section{Mirtha R. Muniz Castillo}

Human Development and Autonomy in Project Aid: Experiences from four bilateral projects in Nicaragua and El Salvador

MGSoG Dissertation Series, № 4

\section{Christiane Arndt}

Governance Indicators

MGSoG Dissertation Series, № 3 


\section{Britta Augsburg}

Microfinance: Greater Good or Lesser Evil?

MGSoG Dissertation Series, № 2

2008

\section{Rutger Daems}

Medicines for the Developing World

UNU-MERIT Dissertation Series, № 54

\section{Johannes Hanel}

Assessing Induced Technology: Sombart's

Understanding of Technical Change in the

History of Economics

UNU-MERIT Dissertation Series, № 53

\section{Rifka Weehuizen}

Mental Capital: the Economic Significance of Mental Health

UNU-MERIT Dissertation Series, № 52

\section{Danielle Cloodt}

The Relationship between RED

Partnership Formation, Social

Embeddedness and Innovative Performance

UNU-MERIT Dissertation Series, № 51

\section{Sabine Fuss}

Sustainable Energy Development under

Uncertainty

UNU-MERIT Dissertation Series, № 50

\section{Geranda Notten}

Measuring and Managing Poverty Risks

MGSoG Dissertation Series, № 1

\section{7}

\section{Tobias Kronenberg}

Reconciling Environmental Conservation with Economic Prosperity: The Feasibility of Double Dividends in the Short and Long Run

UNU-MERIT Dissertation Series, № 49

\section{Viktoria Kravtsova}

Assessing the Impact of Foreign Direct Investment in Transition Economies

UNU-MERIT Dissertation Series, № 48

\section{Suhail Sultan}

The Competitive Advantage of Small and Medium Sized Enterprises: The Case of Jordan's Natural Stone Industry UNU-MERIT Dissertation Series, № 47

2006

\section{Bulat Sanditov}

Essays on Social Learning and Imitation UNU-MERIT Dissertation Series, № 46

\section{Mamata Parhi}

Dynamics of New Technology Diffusion: A Study of the Indian Automotive Industry UNU-MERIT Dissertation Series, № 45

\section{Andreas Reinstaller}

Social Structures and the Innovation

Process: Their Role in the Demand of Firms and Consumers

UNU-MERIT Dissertation Series, № 44

\section{Rose Kiggundu}

Innovation systems and Development: The Journey of a Beleaguered Nile Perch Fishery in Uganda

UNU-MERIT Dissertation Series, № 43

\section{Thomas Pogue}

The Evolution of Research Collaboration in South African Gold Mining: 1886-1933

UNU-MERIT Dissertation Series, № 42

\section{Geoffrey Gachino}

Foreign Direct Investment, Spillovers and Innovation: The Case of Kenyan

Manufacturing Industry

UNU-MERIT Dissertation Series, № 41 


\section{Önder Nomaler}

Technological Change, International Trade and Growth: An Evolutionary, Multi-

Agents-Based Modeling Approach

UNU-MERIT Dissertation Series, № 40

2005

\section{Samia Satti Osman Mohamed-Nour}

Change and Skill Development in the Arab

Gulf Countries

UNU-MERIT Dissertation Series, № 39

\section{Elad Harison}

Intellectual Property Rights: Economics and Policy Analysis

UNU-MERIT Dissertation Series, № 38

\section{Daniel Dalohoun}

Learning to innovate: agricultural innovation and entrepreneurship: the case of Songhaï farmers in Benin

UNU-MERIT Dissertation Series, № 37

\section{Müge Ozman}

Networks, Organizations and Knowledge

UNU-MERIT Dissertation Series, № 36

\section{Bas Straathof}

Product Variety and Economic Growth:

The Counteracting Effects of Scale and

Idiosyncrasy

UNU-MERIT Dissertation Series, № 35

\section{Wilfred Schoenmakers}

Knowledge Flows between Multinational Companies: A Patent Data Analysis

UNU-MERIT Dissertation Series, № 34

\section{Myriam Cloodt}

Mergers and Acquisitions ( $M$ and As) in

High-Tech Industries: Measuring the Post-

$M$ and $A$ Innovative Performance of

Companies

UNU-MERIT Dissertation Series, № 33

2004

\section{Paola Criscuolo}

RED Internationalisation and Knowledge

Transfer: Impact on MNEs and their Home Countries

UNU-MERIT Dissertation Series, № 32

\section{Maarten Verkerk}

Trust and Power on the Shop Floor

UNU-MERIT Dissertation Series, № 31

\section{Gottfried Leibbrandt}

Adoption, Harmonization and Succession of Network Technologies across Countries UNU-MERIT Dissertation Series, № 30

\section{Mark Sanders}

Skill Biased Technical change: Its Origins, the Interaction with the Labour Market and Policy Implications

UNU-MERIT Dissertation Series, № 29

\section{3}

\section{Nadine Roijakkers}

Inter-firm Cooperation in High-tech Industries: a Study of RED Partnerships in Pharmaceutical Biotechnology

UNU-MERIT Dissertation Series, № 28

\section{Viki Sonntag}

Speed, Scale and Sustainability

UNU-MERIT Dissertation Series, № 27 
Masaru Yarime

From End-of-Pipe Technology to Clean

Technology

UNU-MERIT Dissertation Series, № 26

\section{Stéphane Malo}

The Combinatorial Chemistry Revolution:

Sustaining a Superior Performance

Position through Technological Learning

UNU-MERIT Dissertation Series, № 25

2002

\section{Annelies Hogenbirk}

Determinants of Inward Foreign Direct

Investment: the Case of the Netherlands

UNU-MERIT Dissertation Series, № 24

\section{Bastiaan Johan terWeel}

The Computerization of the Labour Market UNU-MERIT Dissertation Series

2001

\section{John Adeoti}

Technology Investment in Pollution

Control in Sub-Saharan Africa: The Case of the Nigerian Manufacturing Industry

UNU-MERIT Dissertation Series, № 23

\section{Edward Huizenga}

Innovation Management: How

Frontrunners Stay Ahead: An Empirical

Study on Key Success Factors in the ICT sector

UNU-MERIT Dissertation Series, № 22

2000

Machiel van Dijk

Technological Change and the Dynamics of Industries: Theoretical Issues and

Empirical evidence from Dutch

Manufacturing

UNU-MERIT Dissertation Series, № 21
1999

\section{Jan Cobbenhagen}

Managing Innovation at the Company

Level: A Study on Non-Sector-Specific

Success Factors

UNU-MERIT Dissertation Series, № 20

\section{Marjolein Caniëls}

Regional Growth Differentials: The Impact of Locally Bounded Knowledge Spillovers

UNU-MERIT Dissertation Series, № 19

1998

\section{Aldo Geuna}

Resource Allocation and Knowledge

production: Studies in the Economics of

University Research

UNU-MERIT Dissertation Series, № 18

1996

\section{Reinoud Joosten}

Dynamics, Equilibria, and Values

UNU-MERIT Dissertation Series, № 17

\section{Hugo Kruiniger}

Investment, $R \mathcal{E} D$, and the Financing

Decisions of the Firm

UNU-MERIT Dissertation Series, № 16

1995

\section{Hans van Meijl}

Endogenous Technological Change: The

Case of Information Technology,

Theoretical Considerations and Empirical

Results

UNU-MERIT Dissertation Series, № 15 


\section{René Kemp}

Environmental Policy and Technical

Change: A Comparison of the Technological

Impact of Policy Instruments

UNU-MERIT Dissertation Series, № 14

\section{Rohini Acharya}

The Impact of New Technologies on

Economic Growth and Trade: A Case Study

of Biotechnology

UNU-MERIT Dissertation Series, № 13

\section{Geert Duysters}

The Evolution of Complex Industrial

Systems: The Dynamics of Major IT

Sectors

UNU-MERIT Dissertation Series, № 12

\section{Marjan Groen}

Technology, Work and Organisation: A

Study of the Nursing Process in Intensive

Care Units

UNU-MERIT Dissertation Series, № 11

1994

\section{Huub Meijers}

On the Diffusion of Technologies in a

Vintage Framework: Theoretical

Considerations and Empirical Results

UNU-MERIT Dissertation Series, № 10

\section{Theon van Dijk}

The Limits of Patent Protection: Essays on the Economics of Intellectual Property Rights

UNU-MERIT Dissertation Series, № 9

\section{Hans Voordijk}

Naar Integrale Logistiek in Bedrijfsketens:

Ontwikkelingen in de Bouw

UNU-MERIT Dissertation Series, № 8
1993

\section{Paul Diederen}

Technological Progress in Enterprises and Diffusion of Innovation: Theoretical

Reflections and Empirical Evidence

UNU-MERIT Dissertation Series, № 7

\section{Ben Dankbaar}

Economic Crisis and Institutional Change: The Crisis of Fordism from the Perspective of the Automobile Industry

UNU-MERIT Dissertation Series, № 6

\section{Hanno Roberts}

Accountability and Responsibility: The

Influence of Organisation Design on

Management Accounting

UNU-MERIT Dissertation Series, № 5

1992

\section{Bart Verspagen}

Uneven Growth between Interdependent

Economies: An Evolutionary View on

Technology Gaps, Trade and Growth

UNU-MERIT Dissertation Series, № 4

\section{Sjoerd Romme}

A Self-organization Perspective on Strategy

Formation

UNU-MERIT Dissertation Series, № 3

1989

\section{John Spangenberg}

Economies of Scale, and Atmosphere in

Research Organisations

UNU-MERIT Dissertation Series, № 2 


\section{John Hagedoorn}

Evolutionary and Heterodox Innovation

Analysis: A Study of Industrial and

Technological Development in Process

Control and Information Technology

UNU-MERIT Dissertation Series, № 1 

